


\section{Image-based endoscope navigation and clinical applications}

Nanda van der Stap 
Graduation committee:

Chairman:

Promotor:

Co-promotor:

Members:

Prof.dr.ir. S. Stramigioli

Prof.dr.ir. C.H. Slump

Prof.dr. T. Ruers

Prof.dr. S. Perretta, MD

Prof.dr. W. Niessen

dr. P. Dik

Referee:

dr. M.P. Schwartz
Prof.dr. P.M.G. Apers

Prof.dr. I.A.M.J. Broeders, MD

dr.ir. F. van der Heijden

University of Twente

University of Twente

University of Twente \& NKI-AVL

University of Strasbourg \& IRCAD-EITS

Erasmus University

UMC Utrecht

Meander medical center

DOI: 10.3990/1.9789036539685

ISBN: 978-90-3653968-5

Publisher: Ridderprint BV

Cover design: Stefan Groothuis \& Nanda van der Stap

Copyright @Nanda van der Stap

\section{MIRA}

BIOMEDICAL TECHNOLOGY AND TECHNICAL MEDICINE

\author{
MIRA Institute \\ Chair of Robotics and Mechatronics \\ University of Twente \\ P.O. Box 217, NL - 7500 AE Enschede
}

Dit proefschrift wordt mede mogelijk gemaakt door:

Olympus Nederland B.V. 


\title{
IMAGE-BASED ENDOSCOPE NAVIGATION AND CLINICAL APPLICATIONS
}

\author{
PROEFSCHRIFT
}

ter verkrijging van

de graad van doctor aan de Universiteit Twente, op gezag van de rector magnificus,

Prof. dr. H. Brinksma,

volgens besluit van het College voor Promoties,

in het openbaar te verdedigen

op vrijdag 22 januari 2016 om 14.45 uur

door

Nanda van der Stap

geboren op 13 oktober 1985

te Heemstede, Nederland 
Dit proefschrift is goedgekeurd door:

Prof. dr. I.A.M.J. Broeders (promotor)

Dr. ir. F. van der Heijden (assistent-promotor) 


\section{Samenvatting}

De vraag naar flexibele endoscopische procedures groeit snel. De veiligheid moet gegarandeerd blijven, terwijl de efficiëntie en toepassingsgebieden vergroot moeten worden. Technologische oplossingen zoals robotisering van endoscopen hebben de potentie om deze eisen in te willigen. Automatisering van de robotische navigatie van flexibele endoscopen heeft een veelbelovende toekomst in endoscopie, zowel voor diagnostische als therapeutische procedures.

Onze groep heeft gewerkt aan verschillende oplossingen voor het robotiseren van sturing. Een prototype robot voor conventionele flexibele endoscopen, zoals nu gebruikt in elk ziekenhuis, is eerder ontwikkeld. De volgende stap is het ontwikkelen van software toepassingen die de navigatie automatiseren en de sturing van de endoscoop verder vergemakkelijken.

Deze dissertatie rapporteert de resultaten van het onderzoek naar (semi)automatische sturing van endoscopen. De haalbaarheid van verschillende software algoritmes is bepaald en hieronder in meer detail beschreven. Allereerst is er een algoritme ontwikkeld gebaseerd op optical flow, om de bewegingsrichting van de flexibele endoscoop te bepalen. Optimalisatie en evaluatie van dit algoritme is uitgevoerd middels in-vitro experimenten. Ten tweede is een detectie algoritme beschreven dat de doelrichting van de endoscoop automatisch kan schatten. Zodra de doelrichting bekend is kan de tip van de endoscoop gecorrigeerd worden door gebruik te maken van het eerder ontwikkelde robotische platform. Als laatste is er een algoritme ontwikkeld, geïmplementeerd en gevalideerd dat de endoscoop kan stabiliseren op een willekeurig doelgebied tijdens interventies.

\section{De bewegingsrichting van flexibele endoscopen}

'Optical flow' is een techniek die kan worden gebruik om de verplaatsing van beeldpunten tussen twee afbeeldingen te bepalen. De cameraverplaatsing kan in theorie worden berekend met behulp van deze verplaatsing. Aangezien de camera van een endoscoop op de tip is gelokaliseerd staat de cameraverplaatsing gelijk aan de tipverplaatsing, oftewel de bewegingsrichting van de endoscoop. Zodra deze bekend is en daarnaast ook bekend is wat de doelrichting is, kan het verschil tussen hen worden berekend en kan hiervoor worden gecorrigeerd.

Echter, zowel de endoscoop als de omgeving zijn flexibel in deze procedures, en de geproduceerde beelden zijn verre van ideaal. Deze factoren compliceren het schatten van de camerabeweging. Een selectie van optical flow algoritmes is daarom geïmplementeerd en vergeleken om de beste oplossing te vinden. Bovendien is er een onbekende relatie tussen de echte beweging (in 3D) en de geprojecteerde verplaatsingen die dit veroorzaakt in het optical flow veld (2D). Validatie-experimenten zijn uitgevoerd om 
inzicht te krijgen in deze relatie.

\section{De doelrichting van flexibele endoscopen}

De doelrichting van de endoscoop kan worden verkregen met een speciaal ontwikkeld lumendetectie-algoritme, dat is gebaseerd op het detecteren van het donkerste gebied. Manuele lumenindicatie is hierbij gebruikt als gouden standaard, en de automatische resultaten zijn vergeleken middels een correlatieanalyse.

Het ontwikkelde algoritme is geïmplementeerd in het robotisch endoscopie systeem en verschillende gebruikersgroepen zijn gevraagd om een gesimuleerde colonoscopie uit te voeren met het systeem. De resultaten zijn veelbelovend; hoewel er verbeteringen in experimentopzet en het systeem zelf nodig zijn, waren de meeste gebruikers positief over het systeem en de toepassingen.

\section{Doelgebied-fixatie in flexibele endoscopie}

Met een combinatie van een tracking algoritme gebaseerd op optical flow en het control algoritme dat is gebruikt voor de lumencentralisatie is een endoscoopstabilisatie algoritme ontwikkeld voor het robotisch platform. De arts selecteert handmatig een doelgebied om het algoritme te initialiseren. Dit heeft als voordeel dat een arts elk willekeurig doelgebied kan kiezen waarop de endoscoop moet fixeren; iets dat met name nuttig is voor therapeutische procedures. Het ontwikkelde algoritme is in-vitro getoetst op robuustheid en nauwkeurigheid in beeldsequenties van endoscopische interventies.

Het tracken van een doelgebied is stap één, maar vervolgens de endoscooptip exact naar het doel sturen is een minstens even belangrijke volgende stap. Endscooptipcorrectie wordt bemoeilijkt door de flexibiliteit van de endoscoop, omdat hierdoor hysterese en andere ongewenste dynamica optreedt. Een mogelijke aanpak om deze correctie uit te kunnen voeren wordt besproken in het laatste hoofdstuk van deze dissertatie.

Samenvattend laten de resultaten hier beschreven zien dat beeldgestuurde navigatie bijdraagt aan de intuitiviteit van flexibele endoscoop sturing. Nieuwe mogelijkheden voor diagnostiek en therapie ontstaan als deze technologie geïncorporeerd kan worden in de klinische praktijk. 


\section{Abstract}

The demand for flexible endoscopic procedures is increasing fast. In addition, the safety needs to be guaranteed while the efficiency and applicability of flexible endoscopes needs to be extended. Technological solutions, including robotized steering of the endoscope, may fulfill these requirements. Automating the robotic navigation of flexible endoscopes is a promising future for endoscopy, in diagnostic as well as therapeutic interventions.

Our group has worked on several solutions for steering robotization. A prototype robotic steering module was developed to enable remote steering of flexible endoscopes that are currently used in clinical practice. The next step is to develop software applications that automate navigation and facilitate steering further.

This thesis reports the results of an investigation towards (semi-)automated endoscope navigation. The feasibility of several software improvements was determined and described below in more detail. First, an optical-flow based algorithm was developed to estimate the motion of the endoscope. Optimization and evaluation of the algorithm was done in vitro. Second, a lumen detection algorithm was developed to determine the target direction. With the target direction known, the tip direction can be corrected using the earlier developed robotized steering platform. Last, an endoscope stabilization algorithm was designed, implemented and validated that can focus on any target area.

\section{Heading direction of flexible endoscopes}

'Optical flow' is the technique that calculates the displacement of image features or pixels between two images. From this displacement, camera motion can theoretically be derived. Since the camera of an endoscope is located at the tip, with this derivation the endoscope displacement is also known. Once endoscope heading direction is known, and it is also known in which direction the endoscope should go (target direction), the difference between them can be computed and compensated for.

However, in flexible endoscopy both the endoscope and the environment are flexible, and the produced images are far from perfect. This makes calculating camera motion from optical flow challenging. A selection from different optical flow algorithms was therefore implemented and compared to determine the best solution. In addition, there is a relation between motion present in the real world (3D) and the resulting projected displacements in the optical flow field (2D) that is not exactly known. Validation experiments were performed to gain insight in this relation. 


\section{Target direction of flexible endoscopes}

The target direction of the endoscope was obtained with a custom-designed lumen detection algorithm based on dark region detection. Manual indication of the lumen was used as the golden standard, and a correlation analysis provided the correspondence between automated and manual results.

The algorithm was implemented in the robotized endoscopy system and different user groups were asked to perform a simulated colonoscopy with the system. The results are promising; although improvements in system and experimental design are still needed, most users were positive about the system and its applications.

\section{Target locking in flexible endoscopy}

Using a combination of an optical flow-based tracking algorithm and the control algorithm from the lumen centralization, an endoscope stabilization system was developed. The physician first selects a Region Of Interest (ROI) manually, to initialize the algorithm. This has the advantage that a physician is able to indicate any area that needs to remain centralized in the image. This functionality will be specifically useful for therapeutic interventions. The algorithm was tested in-vitro on robustness and accuracy in image sequences depicting endoscopic interventions.

Tracking a target is step one, but subsequently correcting the endoscope tip towards the target is the equally important second step. Correcting the endoscope tip is complicated by the flexibility of the endoscope, since it causes hysteresis and other undesired dynamics. A possible approach to correct the endoscope is presented in the last chapter of this thesis.

Summarizing, the results from these studies show that image-guided navigation of flexible endoscopes contributes to steering intuitiveness. New possibilities for diagnostics and therapeutics open up if this technology can be incorporated in clinical practice. 


\section{Contents}

1 Introduction 1

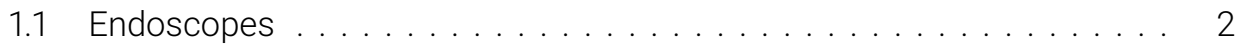

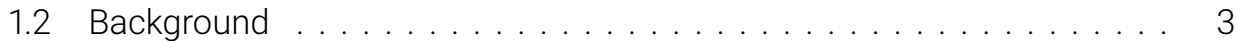

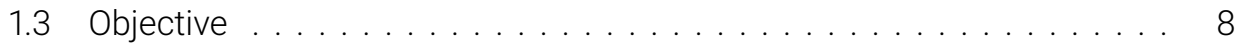

1.4 Contributions .......................... 8

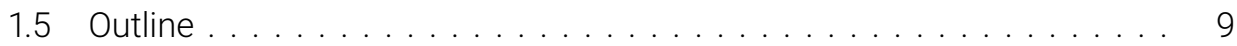

1.6 References .............................. 10

2 Towards automated visual flexible endoscope navigation 11

2.1 Introduction . . . . . . . . . . . . . . . . . . . . . . . . . 12

2.2 Materials and methods . . . . . . . . . . . . . . . . . . . 13

2.3 Results . . . . . . . . . . . . . . . . . . . . . . . . . . . . . . . . 14

2.4 Discussion . . . . . . . . . . . . . . . . . . . . . 20

2.5 Appendix . . . . . . . . . . . . . . . . . . . . . . . . 21

2.6 References . . . . . . . . . . . . . . . . . . . . . . . . 24

3 The focus of expansion in automated flexible endoscope steering 25

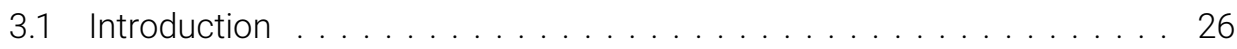

3.2 Endoscope navigation . . . . . . . . . . . . . . . . . . . . 29

3.3 Experimental setup . . . . . . . . . . . . . . . . . . . . . . 31

3.4 Experimental results . . . . . . . . . . . . . . . . . . . . . . . . . . 34

3.5 Discussion . . . . . . . . . . . . . . . . . . . . . . . 34

3.6 Conclusions and future work . . . . . . . . . . . . . . . 36

3.7 References . . . . . . . . . . . . . . . . . . . . 36

4 Comparing optical flow techniques in in-vivo images 37

4.1 Introduction . . . . . . . . . . . . . . . . . 38

4.2 Related work in endoscopy . . . . . . . . . . . . . . . . . . . . . 39

4.3 Approach . . . . . . . . . . . . . . . . . . . . . . 40

4.4 Results . . . . . . . . . . . . . . . . . . . . . . . . . . 49

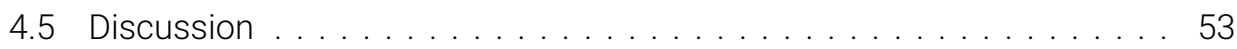

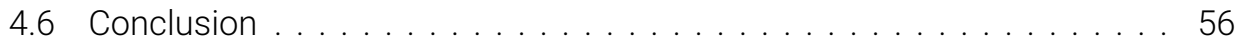

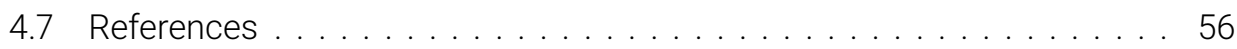


5 Navigation in endoscopy: investigation of an optical flow model 57

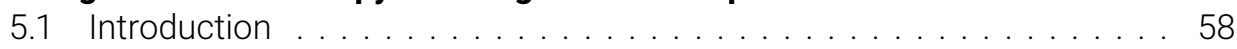

5.2 Methods . . . . . . . . . . . . . . . . . . . . . 62

5.3 Results . . . . . . . . . . . . . . . . . . . . 70

5.4 Discussion . . . . . . . . . . . . . . . . . . . 73

5.5 Conclusion . . . . . . . . . . . . . . . . . . . . . . . 74

5.6 References ............................... . 74

6 Validation of an automated lumen detection algorithm 75

6.1 Introduction . . . . . . . . . . . . . . . . . . . . 76

6.2 Materials and methods . . . . . . . . . . . . . . . . . . 77

6.3 Results . . . . . . . . . . . . . . . . . . . . . . . . . . 81

6.4 Conclusion . . . . . . . . . . . . . . . . . . . . . 84

6.5 References ................................ 85

7 Automated lumen centralization for colonoscopy: user evaluation $\quad 87$

7.1 Introduction . . . . . . . . . . . . . . . . . . 88

7.2 Participants and methods . . . . . . . . . . . . . . . . 88

7.3 Results . . . . . . . . . . . . . . . . . . . . . . . . . . . . . . . 91

7.4 Discussion .......................... 92

7.5 Acknowledgements . . . . . . . . . . . . . . . . . . . 94

7.6 References ............................ 95

8 Technical Validation of Automated Lumen Centralization 97

8.1 Introduction . . . . . . . . . . . . . . . . . . . . 98

8.2 Materials . . . . . . . . . . . . . . . . . . . . . . . 99

8.3 Methods . . . . . . . . . . . . . . . . . . . . . . . . . . . . 102

8.4 Results . . . . . . . . . . . . . . . . . . . . . . . . . . . 104

8.5 Discussion . . . . . . . . . . . . . . . . . . . . . . 106

8.6 Conclusion . . . . . . . . . . . . . . . . . . . . . . . . 108

8.7 Acknowledgements . . . . . . . . . . . . . . . . . . . . . 108

8.8 References . . . . . . . . . . . . . . . . . . . . . . . . . . 108

9 A real-time target tracking algorithm for robotized endoscopy 109

9.1 Introduction . . . . . . . . . . . . . . . . . . . . . . . 110

9.2 Materials and methods . . . . . . . . . . . . . . . . . . . . . . . . 111

9.3 Results . . . . . . . . . . . . . . . . . . . . . . . . . . . . . . 114

9.4 Discussion . . . . . . . . . . . . . . . . . . . . . . . . . . . 114

9.5 Conclusion . . . . . . . . . . . . . . . . . . . . . . . 116

9.6 References ............................... . 116

10 A camera stabilization system for flexible endoscopic interventions 117

10.1 Introduction . . . . . . . . . . . . . . . . . . . . . . . . . 118

10.2 Methods and materials . . . . . . . . . . . . . . . . . . . . . 122

10.3 Results . . . . . . . . . . . . . . . . . . . . . . . . 126

10.4 Discussion . . . . . . . . . . . . . . . . . . . . . . . . . . . 129 
10.5 Conclusion . . . . . . . . . . . . . . . . . . . . . . . . . 132

10.6 References . . . . . . . . . . . . . . . . . . . . . . . 132

11 Conclusions 133

11.1 Summary of findings and conclusions . . . . . . . . . . . . . . . . . . . . . 134

11.2 Recommendations . . . . . . . . . . . . . . . . . . . . . . . . 137

A Camera calibration using magnetic tracking 139

A.1 References . . . . . . . . . . . . . . . . . . . . . . . . . . . . 147

$\begin{array}{ll}\text { Bibliography } & 149\end{array}$

$\begin{array}{ll}\text { About the author } & 161\end{array}$

$\begin{array}{ll}\text { Dankwoord } & 163\end{array}$ 


\section{List of Figures}

1.1 View axes and working axes in different surgical modalities. . . . . . . 4

1.2 Degrees of freedom scheme for endoscopes. . . . . . . . . . . . . . 5

1.3 Various lumen views. . . . . . . . . . . . . . . . . . . 6

1.4 Lumen centralization vs. heading direction determination. . . . . . . . . 7

2.1 General shape of an endoscope . . . . . . . . . . . . . . . . 12

2.2 Tip orientation vs. tip heading direction . . . . . . . . . . . . 14

2.3 Lumen centralization principle. . . . . . . . . . . . . . . . 15

2.4 Relevant situations in the colon for endoscope steering. . . . . . . . . 17

2.5 Depth estimation in endoscopic images. . . . . . . . . . . . . . . . 18

2.6 Optical flow field. . . . . . . . . . . . . . . . . . . . . 18

3.1 Possible endoscopic movement and resulting images. . . . . . . . . . 27

3.2 Example colonoscopy images. . . . . . . . . . . . . . . . . . . 28

3.3 Visualization of manual and automated FOE. . . . . . . . . . . . . . . 29

3.4 Flowchart SIFT feature extraction. . . . . . . . . . . . . . . . 32

3.5 Flowchart SIFT matching. . . . . . . . . . . . . . . . . 33

3.6 Flowchart final estimation using RANSAC. . . . . . . . . . . 33

3.7 Manual vs. automated results FOE computation. . . . . . . . . . . . . 35

4.1 Real colonoscopic vs. anatomic model image. . . . . . . . . . . . . 39

4.2 Benchmark image pairs. . . . . . . . . . . . . . . . . . . . . . . . 41

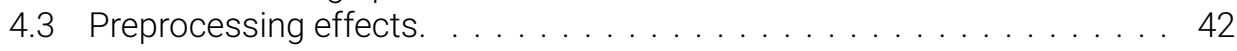

4.4 Manual matching results. . . . . . . . . . . . . . . . . . 47

4.5 Fundamental matrix distance representation. . . . . . . . . . . . . . . . 48

4.6 Optical flow matches and computation times (LK) . . . . . . . . 50

4.7 Color visualization of $\mathrm{F}$ distances $(\mathrm{LK}) \ldots \ldots \ldots \ldots \ldots \ldots \ldots . . \ldots \ldots$

4.8 Visual representation of distances for the SIFT comparison. . . . . . . . 51

4.9 SIFT matches and inliers per distance ratio. . . . . . . . . . . . 52

4.10 Summary outcomes of the SIFT algorithm. . . . . . . . . . 53

4.11 Fraction between matches and inliers for SIFT-LK . . . . . . . . . 54

4.12 Color visualization of F distances (SIFT-LK) . . . . . . . . . . 54

5.1 Endoscope with tip shown enlarged . . . . . . . . . . . . . . 58

5.2 Endoscopic tip correction . . . . . . . . . . . . . . . . . . . . . 61

5.3 Epipolar geometry representation. . . . . . . . . . . . . . . 62

5.4 Visualization of point feature displacement in images. . . . . . . . . . 64 
5.5 Schematic overview of validation experiment setup . . . . . . . . . . 66

5.6 Representation of transformation matrices . . . . . . . . . . 67

5.7 Experimental setup using perspex tube . . . . . . . . . . . . . . . . 69

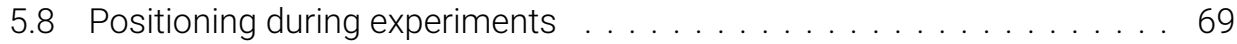

5.9 Experimental setup camera calibration . . . . . . . . . . . . . . . 70

5.10 Reprojection of camera frames. . . . . . . . . . . . . . . . . . 70

5.11 EM tracking results. . . . . . . . . . . . . . . . . . . . . 71

5.12 Results for different environments. . . . . . . . . . . . . . . 72

5.13 FOE results. . . . . . . . . . . . . . . . . . . . . . . 72

5.14 Epipolar geometry results. . . . . . . . . . . . . . . . . . 73

6.1 Dark region segmentation. . . . . . . . . . . . . . . . . . . 76

6.2 Experimental setup. . . . . . . . . . . . . . . . . . . 78

6.3 Control flowchart robotized endoscopy system. . . . . . . . . . . . . 79

6.4 Lumen detection algorithm flowchart. . . . . . . . . . . . . . . . 80

6.5 Manual and automated lumen detection results. . . . . . . . . . . . 83

6.6 Image sequence 5 example. . . . . . . . . . . . . . . . . . . . 84

7.1 Colon segments and different cases. . . . . . . . . . . . . . . 90

7.2 Experimental setup user evaluation. . . . . . . . . . . . . . . . 90

7.3 Box-plot for cecal intubation times. . . . . . . . . . . . . . . . 92

7.4 Polyp detection rates. . . . . . . . . . . . . . . . . . . . . 93

7.5 The Kyoto Kagaku model. . . . . . . . . . . . . . . . . . . . . . . . . . 95

7.6 Close-up view of the robotic steering mechanism. . . . . . . . . . . . 95

8.1 Experimental setup lumen centralization feasibility. . . . . . . . . . . 100

8.2 Enabling automated navigation. . . . . . . . . . . . . . . . . . 100

8.3 Control loop for endoscopic system. . . . . . . . . . . . . . . . . . . . 101

8.4 Implemented lumen centralization algorithm flowchart. . . . . . . . . . 101

8.5 Different case configurations anatomic model. . . . . . . . . . . . . . 102

8.6 User view during experiments. . . . . . . . . . . . . . . . . . . . . 103

8.7 Automated centralization ON. . . . . . . . . . . . . . . . . . . . . . . 104

8.8 Mean duration of automated navigation use. . . . . . . . . . . . . 105

8.9 Manual vs. automated lumen results with ON-time. . . . . . . . . . . 107

9.1 Polypectomy example image. . . . . . . . . . . . . . . . . . . . 113

9.2 Distance comparison plot with causes. . . . . . . . . . . . . . . 115

10.1 Schematic endoscope. . . . . . . . . . . . . . . . . . . . . . . . . . . 119

10.2 Hysteresis measurement setup. . . . . . . . . . . . . . . . . . . . . 120

10.3 Position plot showing endoscopic hysteresis. . . . . . . . . . . . . . 121

10.4 Two cables influencing each other. . . . . . . . . . . . . . . . . . . 123

10.5 Setup OptiTrack measurement. . . . . . . . . . . . . . . . . . . . 123

10.6 KUKA arm with LEDs. . . . . . . . . . . . . . . . . . . . . . . . . . . . . 124

10.7 Target motion with respect to tip. . . . . . . . . . . . . . . 126

10.8 Simulation result one cable. . . . . . . . . . . . . . . . . . . . 126 
10.9 Simulation and measurement of tracking. . . . . . . . . . . . . 127

10.10Two cables influencing each other. . . . . . . . . . . . . . . . 128

10.11 Hysteresis compensation effects. . . . . . . . . . . . . . . 128

10.12Tracked horizontal target motion. . . . . . . . . . . . . . . . . . . 129

10.13Tracked vertical target motion. . . . . . . . . . . . . . . . . . 130

10.14Tracked circular target motion. . . . . . . . . . . . . . . . . . . 131

A.1 Coordinate frame configurations . . . . . . . . . . . . . . . . 141

A.2 Camera position during calibration data acquisition . . . . . . . . . 142

A.3 Corner point indication path . . . . . . . . . . . . . . . . . . . . . 143

A.4 Axis definition in images . . . . . . . . . . . . . . . . . . . . . . 144

A.5 Extrinsic camera parameter results . . . . . . . . . . . . . . . . 145

A.6 Transformation definitions . . . . . . . . . . . . . . . . . . . . 145

A.7 Example validation setup (schematic) . . . . . . . . . . . . . . . . 146

A.8 Camera position comparison . . . . . . . . . . . . . . . . . . 147 


\section{List of Tables}

2.1 Content overview of included papers . . . . . . . . . . . . . . . . . 21

2.1 Content overview of included papers . . . . . . . . . . . . . . 22

2.1 Content overview of included papers . . . . . . . . . . . . . 23

4.1 Properties of image pairs for OF evaluation . . . . . . . . . . . 40

4.2 Possible combinations of feature detectors and flow methods . . . . . . . 45

4.3 Outcome for different image pairs with LK . . . . . . . . . . . . . 49

4.4 Outcome for different image pairs with SIFT . . . . . . . . . . . . 51

4.5 Outcome for different image pairs with SIFT-LK . . . . . . . . . . 53

6.1 Properties of used image sequences . . . . . . . . . . . . . . . . . . 82

6.2 Results of automated target detection on image sequences . . . . . . 82

7.1 Questionnaire results. . . . . . . . . . . . . . . . . . . . . . . 94

8.1 Time comparison per colon segment . . . . . . . . . . . . . . 106

9.1 Image sequence properties. . . . . . . . . . . . . . . . . . . . . . . 112

9.2 Endoscope properties. . . . . . . . . . . . . . . . . . . . 113

9.3 Image sequence ICC and RMSE results . . . . . . . . . . . . . . 115

10.1 Measurements done for validation. . . . . . . . . . . . . . . . . . . 125 


\section{Introduction}

Unknown

Part of this chapter is adapted from: N. van der Stap, F. van der Heijden \& I.A.M.J. Broeders (2013). Challenges in navigational strategies for flexible endoscopy. In: Proceedings of the 27th International Congress and Exhibition on Computer Assisted Radiology and Surgery, CARS 2013, 26-29 jun 2013, Heidelberg, Germany. pp. 199201. Springer Verlag. ISSN 1861-6410.

This chapter will introduce the contents of this thesis. 


\subsection{Endoscopes}

Not too long ago, the phrase 'big surgeon, big cut' was commonly heard in surgical education. Young surgeons were advised to make an incision large enough to have adequate access to the operative field; they would need a good overview and enough space for their hands. Over the last few decades, starting in gynecology in the 1960s1970s, the trend has reversed. Patients benefit from small incisions: they have less pain, less wound infections and are able to resume normal life sooner [1]. Technology was developed that offered surgeons the possibility to reduce the dimensions of incisions. Elongated instruments with which they could operate without having their hands at the surgical site directly came to the market. Long tubes called 'trocars' with a small diameter (5-12 $\mathrm{mm}$ ) were developed to guide these instruments through the skin. The elongated instruments are equipped with tips that can cut, manipulate or otherwise interact with tissue, enabling a wide range of surgical procedures.

However, next to their hands, surgeons need their eyes at the surgical site. An interesting piece of technology was developed for this as well. Without endoscopes, so-called minimally invasive surgery would not be possible. The word 'endoscope' is derived from the ancient Greek words 'éndon', which means 'inner', or 'internal', and 'skopéo', which means 'to see'. Endoscopes are nowadays literally used 'to see the internal'. Only a few small incisions are made to enter a set of trocars, a rigid endoscope is inserted through one of them and small instruments go through the other trocars (see Figure 1.1 for an impression of this type of surgery).

Flexible endoscopes exist as well. They are long tubes with a camera at the end, or tip, whereas they can be controlled at the beginning. This configuration makes flexible endoscopes extremely suitable for inspection of, or interventions in, small cavities such as stomach, bowel or lungs. The direct view on the tissue provides information to the physician that has not been equaled by non-invasive imaging techniques (magnetic resonance imaging, computed tomography) to date. In addition, one instrument of a few millimeters can be inserted through the working channel, enabling the taking of tissue samples or even small therapeutic procedures.

Although the design of endoscopes has not changed significantly for decades, the digital age has brought advances to the field of endoscopy. Endoscope use led to the need for monitors in the operating room. Augmented reality in surgery, enabled by additional imaging information, is an emerging field of research. In flexible endoscopy, which was up till recently mainly used for inspection of organs, digitization led to automatic lesion inspection. One of the most notable examples is automated polyp detection in colonoscopy. Where an endoscopist needed to inspect every fold very carefully, it is now possible to receive an (audio)visual aid during colonic inspection.

However, the trend of reducing the invasiveness of surgical procedures has pushed surgery even further. Robot-assisted surgery currently is the state of the art [2], a fact that is emphasized by the current presence of more than 20 robotic surgical systems in the Netherlands only. In 2009, the University of Twente (UT) and mechatronics company Demcon started the robotization of flexible endoscopes with the aim to enable more complex minimally invasive procedures. Then, scar-less surgery through natural orifices (NOTES, natural orifice transluminal surgery) was an upcoming technique, but 
the difficult endoscope manipulation was a limiting factor in the development thereof. With the developed system, called 'Teleflex', Demcon and the University of Twente wanted to solve that problem.

In this thesis, all of the mentioned aspects are combined. Digital imaging technology is used to improve and automate navigation of robotized flexible endoscopes using the Teleflex system. The current chapter introduces some of the principles that are needed to understand the contents of the other chapters. First, the technological aspects of endoscopic surgery and flexible endoscopy are elaborated on. In the following, an overview of possible clinical applications is provided. Last, the objectives, contributions and structure of this thesis are outlined.

\subsection{Background}

An endoscope consists of a rigid or a flexible tube with a lens (system) and a charged coupled device (CCD) chip at the tip. Rigid endoscopes are controlled manually, whereas flexible endoscopes are equipped with turning knobs at a handle that can be used to bend the tip. This way, the endoscope can be maneuvered through the tortuous organs of the human body. The differences between these two types of endoscopes have consequences for the development of (image-guided) robotic platforms.

\subsubsection{Rigid endoscopes}

A rigid endoscope is manually controlled by a camera man and is stiff; not able to bend. This is the type of endoscope most commonly used in surgery. The rigidity provides the advantages of a straight line of sight to the surgical area and robustness against external forces. However, a disadvantage is the decoupling of the line of sight with respect to the working axis (Figure 1.1). In addition, the fulcrum at the position where the trocar enters the patient causes a non-intuitive side-effect: when the surgeon moves his hands to the left, the camera goes to the right and vice versa. Another disadvantage is that providing a stable view is difficult; small hand motions of the camera holder cause large view distortions.

\section{Robot-assisted surgery}

Robot-assisted surgery (RAS) has been introduced in 1994 with the introduction of robotic camera holders. These camera holders provided a stable view, since robots have the advantages of high precision, repeatability and endurance over human assistants $[3,4]$. The camera holders evolved into robotic surgical platforms further on in the 1990's [2]. These platforms have the capability of correcting the fulcrum effect and hand tremor by software adjustments, making performing endoscopic surgery intuitive again.

In CLS, the surgeon has direct control - and thus tactile feedback - over his instruments. These instruments are not always positioned on the same working axis as in open surgery, depending on patient position and the area of interest. Moreover, the surgeon has an indirect view on the operating field with the images of the camera on a distant monitor. The camera is controlled by an assistant and not always accurately 


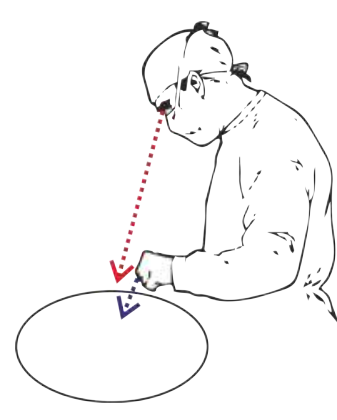

open surgery

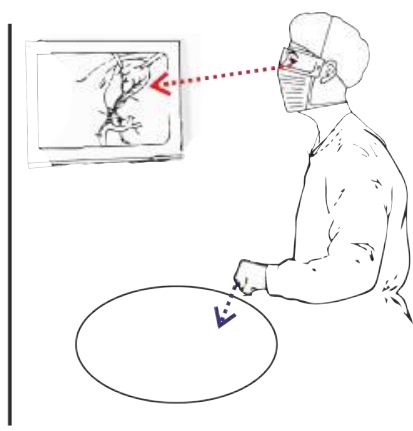

endoscopic surgery

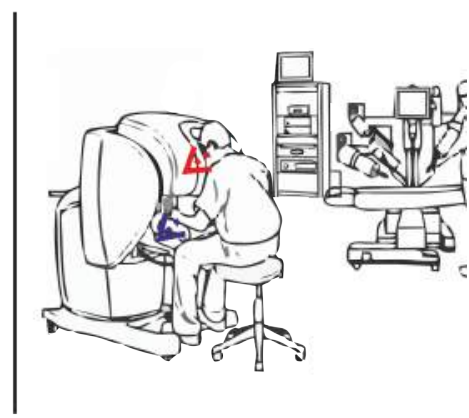

robot-assisted surgery

Figure 1.1: View axes (red) and working axes (blue) for open, endoscopic and robot-assisted surgery. In open and robot-assisted surgery, the axes are aligned, whereas in laparoscopic surgery they are decoupled.

positioned according to the surgeon's ideal visual axis [5]. In RAS, the working axis and visual axis have been restored, as has three-dimensional vision. The surgeon sits in a console while receiving surgery images right in front of his eyes, and is therefore isolated from the environment. The camera is held by the robot and the surgeon has to interrupt the surgery shortly to be able to control the camera.

Robot-assisted endoscopic surgery thus aligns the working axis and visual axis again and gives the surgeon the opportunity to control his own field of view. Most disadvantages of endoscopic surgery are diminished by the advent of this robot, and it provides many advantages in terms of intuitiveness and ergonomics [6]. Moreover, technologically speaking robotizing rigid endoscopes is relatively easy. The stiffness of the scope enables positioning predictions, and if the robot actuates a movement, the endoscope will end up very precisely at the desired position.

\section{Image-guided surgery}

Endoscopic surgery also gave rise to an indirect, digital view of the operating field. With this digitization of the images, new possibilities for surgical guidance opened up. Image processing can be used to track surgical fields of interest, or to provide the surgeon with extra information from preoperative images [7]. However, problems are still encountered in the development of these technologies. The question of clinical relevance is often asked, and technological problems are mainly tissue deformation-related [8]. Tissue deformation is caused by organ motion, patient motion and instrument motion, and it is extremely difficult to predict the resulting deformation robustly. The adoption of image-guided surgery therefore is, in contrast to robotic surgery, relatively low.

\subsubsection{Flexible endoscopes}

When the Teleflex project started in 2009, aim was to apply the technologies used for rigid endoscopes to flexible endoscopes. However, to reach that goal, a completely 
different approach was needed. Flexible endoscopes are also controlled manually, but remotely, using two large steering knobs at the handle (Figure 1.2, right). Most endoscopes have one working channel for instrument insertion, making inspection the endoscope's main feature. Still, the trend of minimizing surgery caused surgeons and endoscopists to experiment with larger and more complex interventions. The instruments therefore needed to handle higher forces and to provide more dexterity. Combined with the non-intuitive and non-ergonomic steering of these endoscopes, it was a logical step for multiple research groups to explore the robotization of flexible endoscopes $[9,10,11]$.

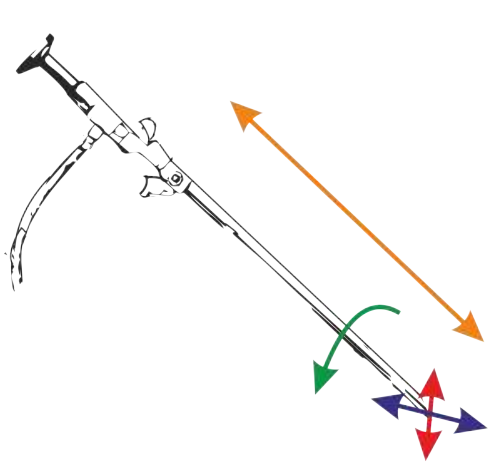

rigid endoscope

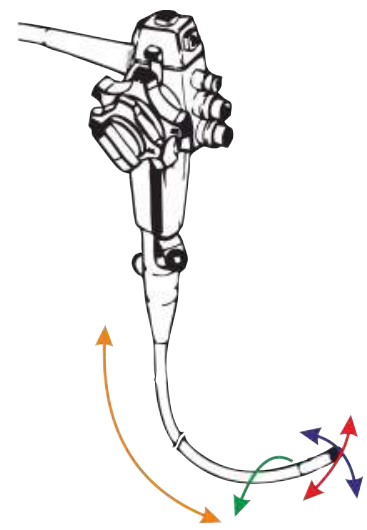

flexible endoscope

Figure 1.2: Degrees of freedom (DOFs) for rigid and flexible endoscopes. Note the curved movement directions for flexible endoscopes.

However, as can be understood from Figure 1.2, robotizing flexible endoscopes is not as straightforward as robotizing rigid ones. The flexibility of the endoscope and the tortuosity of the environment led to complex dynamical interactions. Controlling the tip of the endoscope is already a difficult task; more difficult than for rigid endoscopes because of the flexible directions of movement. The endoscope itself suffers from hysteresis, which means the response to a manipulation command is hard to predict. In addition, the flexibility makes the endoscope and its instruments extremely sensitive to external forces. The main problem when robotizing endoscopes is that these unpredictable factors cause an unknown tip and instrument position, making automating endoscope control very challenging.

\section{Image-guided surgery with flexible endoscopes}

Comparable to endoscopic surgery with rigid endoscopes, image-guided surgery with flexible endoscopes is also explored. However, the presence of the camera on flexible endoscopes provides another application. Since the camera is located at the tip, feedback from the images of the camera can be used to improve endoscope control. This feature comes in handy for the robotization of the flexible endoscope, because one of 
the main problems there is caused by the unknown tip position.

Previous work on this subject has been done [9]. One of the main recommendations from this work was to develop new image-based steering algorithms for the robotized flexible endoscope platform that are robust against illumination changes, artifacts and large motions. This is the objective of the current thesis.

Several research groups have tried to develop image-based flexible endoscope steering using the technique of dark region detection or lumen centralization (see Chapter 2). As can be seen in Figure 1.3, the center of the lumen (C) is the deepest area of the environment, and mostly appears as a darker area in the images. Depth or intensity information obtained from images helps to identify the deepest or darkest part of the image.

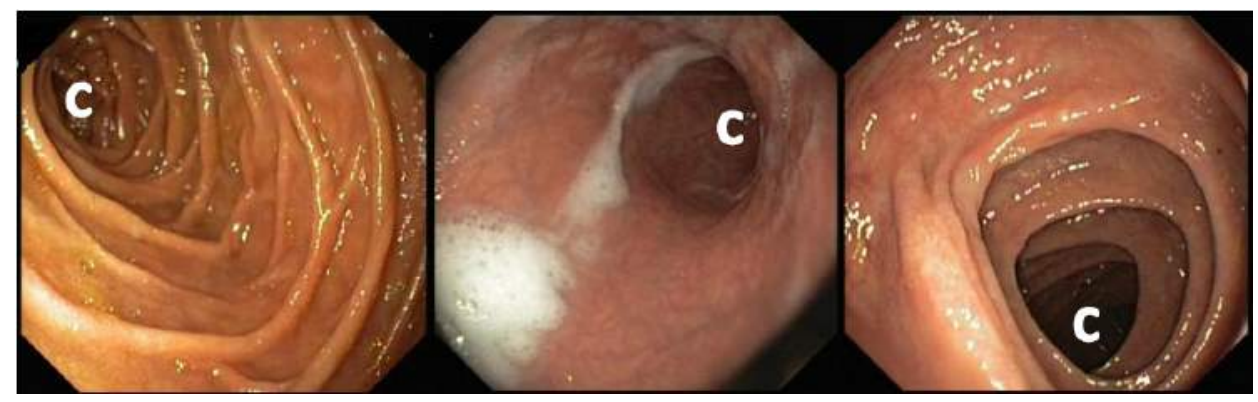

Figure 1.3: Images produced by flexible endoscopes depicting a variety of lumen views.

Endoscope steering is subsequently based on keeping the lumen center centralized in the image. It is assumed that by doing so, the endoscope will travel towards the center when the endoscope is inserted.

However, the lumen centralization approach implies the assumption that the endoscope always travels in the viewing direction of the camera. As the endoscope is flexible, this is not always the case (Figure 1.4). As will be demonstrated in the current thesis, next to a target direction we need a heading direction. Image-based steering algorithms can further be used during therapeutic interventions. The difference between flexible endoscopy in diagnostics and interventions will be explained in the next sections.

\subsubsection{Clinical applications of flexible endoscopes}

Flexible endoscopes are used in colorectal cancer screening as well as endoluminal interventions. Screening programs for colorectal cancer have started in several countries including the Netherlands $[12,13,14]$, and endoscopists are exploring the boundaries of complex interventions. Technological solutions may help to meet the increased demand for flexible endoscopic procedures by increasing the safety and efficiency of these procedures. 


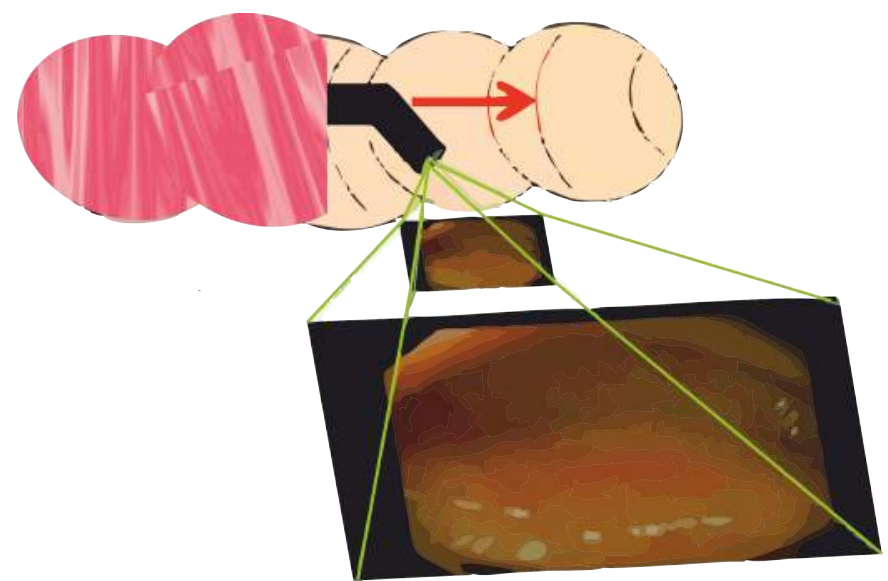

Figure 1.4: Situation in a colonoscopy procedure. The tip of the flexible endoscope is bent and the camera produces a wall view. Lumen centralization will lead to the conclusion that the tip direction has to be corrected. However, the endoscope is already traveling towards the lumen center and does not have to be corrected.

\section{Screening for colorectal cancer}

The direct view on the tissue as well as the tissue sampling possibility of flexible endoscopes makes them the technology of choice for colorectal cancer screening. During these diagnostic procedures, the endoscope is inserted as quickly as possible from the rectum to the cecum, the beginning of the colon in the human body. Then, the endoscope is retracted slowly and rotated continuously to inspect all the tissue in the colon for signs of disease. If such a sign is found, a biopsy can be taken to investigate the underlying pathology. If a relatively small lesion is found, it even can be resected on the spot using the single working channel of the endoscope. Especially these last two features provide endoscopy with a significant advantage over imaging or so-called 'capsule endoscopy', during which the patient swallows a camera pill [15].

Nonetheless, vital disadvantages of the endoscopes still drive the search for improving the technology [16]. The steering mechanism is non-intuitive and non-ergonomic, and it takes 100-300 procedures to reach full competency [17, 18]. If the steering mechanism would be more intuitive, the learning curve is expected to shorten as well. This way, less-trained personnel can be employed in the clinic, effectively reducing the discrepancy between supply and demand for diagnostic colonoscopies.

\section{Interventions}

In an attempt to prevent a larger surgical intervention, the flexible endoscope is more often used to remove increasingly large lesions from the gastrointestinal tract. Examples of procedures include endoscopic submucosal dissection (ESD), during which a lesion is lifted, circumcised and removed using the single instrument channel. Another example is the endoscopic mucosal resection, where a lesion is often taken out piece- 
wise. These procedures can easily take up to two hours to perform. The reason that even skilled endoscopists need such a long time is that controlling the camera and the instruments simultaneously is difficult for the user.

For 'colonoscopically unresectable polyps', sometimes a combination procedure using flexible and rigid endoscopes is employed [4]. As stated earlier, robotization is another option that is researched extensively. Both solutions indicate that the minimal invasive trend is irreversible, and technological improvements are needed to help it continue.

\subsection{Objective}

The main objective of this thesis is to develop and validate image-based methods for automated flexible endoscope navigation with a constant focus on clinical relevance and robustness against artifacts and tissue deformation.

Automating flexible endoscope navigation could lead to an increase in patient safety for endoluminal therapeutic procedures. Additionally, it may decrease the costs of diagnostic flexible endoscope procedures by shortening the learning curve and increasing the efficiency of insertion. Finally, the endoscopist may benefit from ergonomic advantages.

\subsection{Contributions}

Earlier attempts at automating flexible endoscope navigation have not (commercially) succeeded yet. It is assumed that this is mainly due to false premises about the dynamic interaction between the endoscope and the environment. In the current research, the following is contributed to the field:

- A novel approach to flexible endoscope navigation with respect to the environment during diagnostic procedures, consisting of:

- Adaptation of a motion estimation model to the flexible endoscopy application

- Methodology for application-specific yet objective comparison of optical flow techniques

- Validation of the motion estimation model in an in-vitro environment

- Development of a real-time functioning automated lumen centralization algorithm

- First user feedback and feasibility results on the implemented algorithm in the Teleflex system

- Development, implementation and validation of target locking functionality for therapeutic procedures with a robotized flexible endoscope system:

- Development and validation of a real-time functioning target tracking algorithm 
- Experimental validation of the implemented algorithm using the Teleflex system

\subsection{Outline}

The rest of this thesis consists of Chapters 2 - 9 in which the results of the conducted research within the project are presented. In Chapter 10, the findings are related to the previously mentioned research objectives, which concludes this thesis. In the next sections, a short chapter overview is provided.

\subsubsection{Chapter 2: Navigation approach}

In Chapter 2, a literature overview is given on visual navigation in flexible endoscopy. Two main approaches are identified: visual odometry (measuring the traveled path of the endoscope) and lumen centralization (determining the target direction of the endoscope). We found that combining the knowledge of the traveled path with the knowledge of the target location shows potential of solving the navigational problems in automating flexible endoscopy.

\subsubsection{Chapters 3-5: Heading direction estimation}

In Chapters 3 - 5, we investigate the feasibility of a new approach: optical-flow based endoscope steering. 'Optical flow' is the technique that calculates the displacement of image features between two images. From this displacement, the traveled path and camera motion can be derived (up to an unknown scale factor). If this camera motion is known, and the target position is known, a more accurate and robust navigation strategy for flexible endoscopes is within reach.

In this application field not only the endoscope, but also the environment is 'flexible' and unknown. This makes calculating camera motion from optical flow challenging. There is an unknown relation to what motion is present in the real world (3D) and what displacements this motion causes in the optical flow field (2D). It is known however, that when the camera makes a translational motion, the optical flow field produces a radially outward pattern. The center of this pattern, the Focus of Expansion (FOE), then corresponds to the current heading direction of the camera, as demonstrated in Chapter 3.

In Chapter 4, different optical flow algorithms were used to analyze image displacement on test sequences of varying difficulty. This was done to establish the best preprocessing steps and settings of each algorithm. The settings that led to the most accurate optical flow results were accepted as the standard settings of the algorithm for our application. Hereafter, the overall best performing optical flow algorithm could be determined.

The model that was adapted in Chapter 3 still does not explain what happens when the camera rotates. Therefore, the image sequences, produced during a simulated colonoscopic procedure, are recorded together with electromagnetic (EM) tracking data. From the EM data, the relation between 3D (translational and rotational) motion 
and the corresponding optical flow field can be obtained. The goal is to find the optimal optical flow algorithm to derive camera motion from the endoscopic images and quantify robustness and accuracy. Results of this research are described in Chapter 5 .

\subsubsection{Chapters 6-8: Lumen centralization}

Chapters 6 - 8 are dedicated to target direction determination, or lumen centralization. An assisting lumen centralization algorithm was developed and validated (Chapter 6). This algorithm performed well, and was insensitive to artifacts and motion blur compared to previous algorithms. Next, the developed algorithm was implemented in the system developed earlier by the UT and Demcon. Expert endoscopists and novice endoscope users were asked to perform a colonoscopy on a plastic model. Goal of this research was to establish the technical and clinical feasibility of the complete lumen centralization functionality. The user evaluation is described in Chapter 7 , whereas the technical feasibility is described in Chapter 8 .

\subsubsection{Chapters 9-10: Target locking}

As was mentioned in section 1.2.3, controlling the camera (which is located at the tip of the endoscope) and the instruments of a flexible endoscope simultaneously is difficult for the user. However, once camera motion is measurable by optical flow, and the system is remotely controllable, camera steering can be automated. The detected motion of the camera can be automatically corrected for by the robotic system. A custom-designed real-time tracking algorithm was validated as described in Chapter 9. In Chapter 10, the results of the algorithm implementation and complete system validation are elaborated on.

\subsection{References}

Please refer to the Bibliography at the end of this thesis. 
CHAPTER 2

\section{Towards automated visual flexible endoscope navigation}

Isaac Newton

Published as: N. van der Stap, F. van der Heijden \& I. A. M. J. Broeders (2013). Towards automated visual flexible endoscope navigation. Surgical Endoscopy, 27(10), 3539-47. ISSN 0930-2794. doi:10.1007/s00464-013-3003-7.

Reprinted with permission.

A literature study was done with the aim to list currently available solutions for automated, image-based flexible endoscope navigation. The solutions were all solving the navigation problem during the diagnostic phase of the endoscopic procedure. This chapter reports the results of that literature study. 


\subsection{Introduction}

Flexible endoscopes are used in a variety of clinical applications, both for diagnosis and therapy. Not much has changed in flexible endoscopy design for the past fifty years, apart from miniaturization of the cameras [19]. Flexible endoscopes come in various lengths and thicknesses, making them suitable for examining almost any hollow, tubelike structure in the human body [20]. Examples include the bowel, stomach, gall ducts, lungs, and even the salivary glands [21] and brain [22]. The most commonly performed procedures are oesophagogastroduodenoscopy (gastroscopy) and colonoscopy [20]. Generally, a flexible endoscope consists of a long, flexible tube with a light source with and a lens on the tip (Figure 2.1). A CMOS or CCD chip and a video processor are used to convert the light signal to an image. The chip is mostly localized directly at the tip.

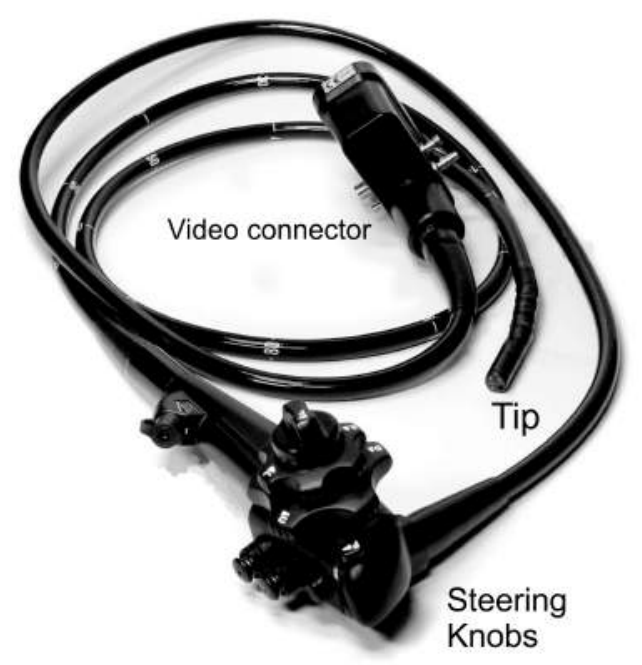

Figure 2.1: The general shape of a flexible endoscope.

The endoscope is inserted in the organ of choice, mostly through a natural orifice. The steering mechanism is generic, but the level of complexity of the environment differs between each organ, putting different requirements on the flexible endoscope. Many endoscopists consider endoscope steering mechanisms non-intuitive. Procedural challenges and physical complaints due to non-ergonomical design are common $[23,24,25]$.

Flexible endoscopes also are the technology of choice for Natural Orifice Transluminal Endoscopic Surgery (NOTES), one of the latest trends in minimally invasive surgery. Examples include the Anubis-scope, the R-scope, the Direct Drive system and the EndoSamurai [11]. Steering and control of the instruments are challenging in these devices. Additionally, screening programs for colorectal cancer require technological solutions to increase the efficiency of endoscopy procedures. Both an expansion of 
complexity in intraluminal interventions and an increasing demand for endoscopy procedures in general have moved scientists to research possibilities for automated steering of these endoscopes.

Robust navigation algorithm development could help in the automation of flexible endoscope steering. The steering can be subdivided in an actuator (the mechanical steering action) and a sensor part (steering control feedback or navigation). In this paper the focus will lie on developing a navigation tool for flexible endoscopes, which is the sensor part. To navigate in any environment, knowledge about the environment itself - where can I go without problems? - , about the current direction - where am I heading now? - , and about the target - where do I want to end up? - are indispensable. Information about the environment might be obtained using either prior knowledge of the anatomy or current images [8]. Assumptions based on pre-procedural data (prior knowledge from CT or MRI scans, for instance) only hold during the procedure itself if the organ(s) are stationary during this procedure. This is often not the case in the highly deformable organs investigated by flexible endoscopes. A bronchoscopy and a ventriculoscopy are examples of exceptions to this rule. Moreover, organ anatomy varies largely among patients, which means that even without considering organ elasticity, it would be very hard to develop a predictive model of these organs. One of the most important requirements for an automated steering technique therefore is: dealing with an unpredictable environment.

Sensing in flexible endoscope systems is often done using the images made by the endoscopy system. This technique is referred to as 'visual navigation'. In this review we aim to describe solutions for the flexible endoscope steering problem based on visual navigation. A subdivision of two main techniques can be made. The first one is based on keeping a certain target in the center of the image. Mostly, the center of the lumen is this target, so we will refer to this technique as lumen centralization. Visual odometry $[26,27]$ is an alternative technique for automated steering. This technique is based on automatic detection of (virtual) landmarks, and subsequently tracking them in the next image. A more elaborate explanation will be provided in the Results section.

\subsection{Materials and methods}

A systematic literature database search was performed in September 2012. Databases that are known for their medical and technological contents were selected. These were Scopus and Medline. Search terms were "flexible endoscopy' AND navigation" (11 results), "automat* AND 'flexible endoscopy"' (10 results) and "application AND 'flexible endoscope"' (114 results). The results were obtained from unrestricted searches over all literature for these words in the article content, title or abstract. The abstract, introduction and conclusion were read, unless exclusion criteria were satisfied within one of these sections. Papers were included if more than one of the following elements were present: full text in English, a flexible endoscopy procedure, an endoscopic platform using flexible endoscopes, an image-guided endoscope application or the presentation or testing of a steering mechanism that partly steers automatically. Of the papers that described a complete automated image-based endoscopic navigation system, the 
'cited by' function of Scopus was used as well (13 results).

\subsection{Results}

26 papers were ultimately included using the inclusion criteria. They were categorized in either lumen centralization or visual odometry and read for advantages and disadvantages of the described technique(s). The general theory about both approaches is explained first, before research details of lumen centralization and visual odometry will be discussed.

\subsubsection{Concepts in visual navigation for automated endoscope steer- ing}

Most navigation systems for flexible endoscopy use endoscope images as sensory input. In endoscope navigation, a distinction can be observed between the tip orientation and the tip (heading) direction (Figure 2.2).

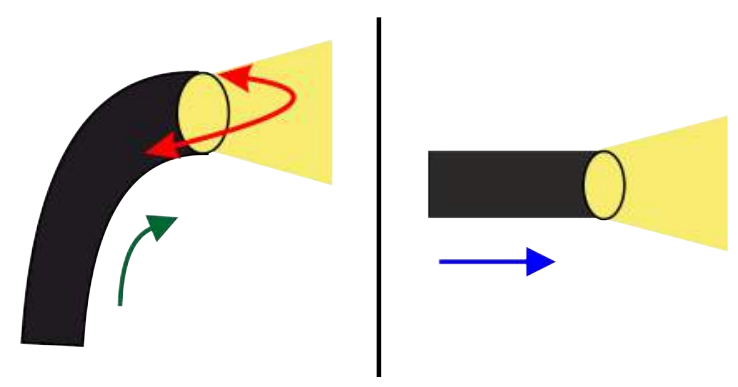

Figure 2.2: The difference between tip orientation and tip (heading) direction is that the first refers to the tip pose, and the latter to the tip motion.

The first refers to the actual pose of the tip and can be expressed in degrees curvature with respect to a reference. The latter (tip direction) refers to the direction in which the tip is traveling, or where the tip will end up if no steering action is undertaken, and can be expressed in image or Euclidian coordinates.

In lumen centralization, finding a target for an endoscope can be achieved with properties of the lumen shown on the images. The center of the lumen is the deepest area of the environment, and mostly appears as a darker area in the images. Depth and image intensity can be discriminated independently on images, which makes them suitable for target finding. Steering is based on keeping the target in the center of the image (Figure 2.3).

This way, the endoscope will travel towards the center when the endoscope is inserted. This technique is mainly used to determine the target and influence tip direction.

With visual odometry, motion between the images can be obtained. In the case of 


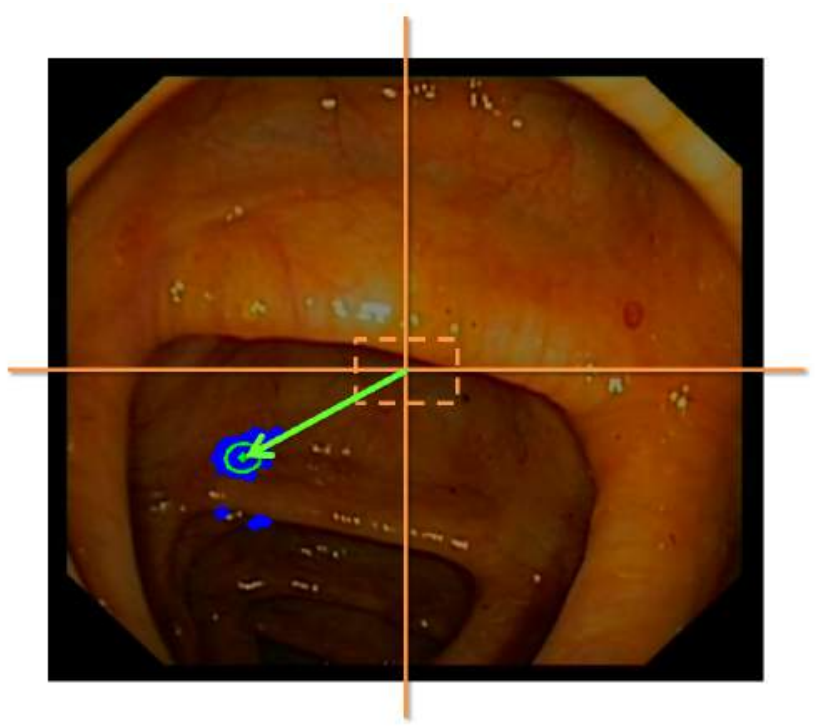

Figure 2.3: Detected lumen (irregular area) is corrected to the center (rectangle in the middle). The arrow indicates steering direction.

flexible endoscopy, this motion corresponds to camera displacement and therefore to endoscope displacement since the camera is located at the tip. Virtual landmarks not only provide environment information, but can be used to define a target as well using information about their location, relative distance or intensity properties for instance. Thus, this technique may be used to provide information about tip orientation and tip direction.

\subsubsection{Research in automating flexible endoscopy}

\section{Lumen centralization}

An automated navigation and advisory system for endoscopes based on lumen centralization was described in 1996 [28]. Dark region segmentation was used to find the central lumen area of the colon. Visible contours of the bowel wall were used as an indication for tip orientation. Dedicated hardware facilities were assembled to enable real-time endoscope steering. System reliability was demonstrated in anatomical (in vitro) models. No experiments using an in vivo model were described. The researchers from [28] published in 1999 [29] on additional techniques to support the dark regionbased lumen centralization. Chettaoui [30], Bricault [31], Reilink [32, 33], Zhiyun [34] and Zhen [35] all used a variety of methods to find the darkest region on endoscopy images. Chettaoui et al. [30] even described a way to differentiate the lumen from diverticula. Especially for fully automated steering systems for colonoscopes and gastroscopes, 
such distinctions may become very important. Automated detection of edges or contours that surround the central lumen area is another approach in the lumen centralization technique $[36,37,38]$.

The lumen center is not only the darkest, but also the deepest part in the image. Finding this part of the image without using dark region detection requires information about the depth of the visible scene. Several techniques are used in the literature to perform depth estimation within human organs. 'Depth estimation' is defined as calculating the three-dimensional (3D) relief or 3D reconstruction of the environment, using the monocular (one lens) camera images produced by the endoscope. Generally, a pixel close-by will appear brighter than a pixel that is far away. This allows calculating depths from a single image.

Structured light is an 'active method' to obtain depth information from a scene [39, 40]. The idea is to project a known pattern onto a surface, and to obtain images of this pattern using a camera. Pattern deformations are caused by depth irregularities in the surface. Analysis of these pattern deformations lead to an accurate and robust method for depth estimation. This technique is applied in industrial endoscopes, but was not found to be applied in clinical endoscopes. Zhang et al. [41] describe the essentials and possibilities of the technology very clearly.

'Shape from shading' is another depth estimation technique used in flexible endoscopy $[28,31,42,43]$. This technique is based on reflecting properties of the surface. In endoscopy, the light source and the observer are in the same plane. This property makes it possible to invert the reflectance equation and to reconstruct the 3D surface coordinates from this [44].

Disadvantages of lumen centralization Although research has shown that lumen detection is feasible for colonoscopic navigation, steering towards the target (the centralization) still poses difficulties. None of the mentioned techniques has successfully been applied in an in vivo situation. The main assumption in all techniques is that by centralizing the lumen center, the endoscope will travel the right path through the organ. Artifacts like residual organ or rinsing fluids cause difficulty in image interpretation. However, the lumen center is not always obtainable from an image. Moreover, multiple forces - intra-abdominal pressure from the patient, insertion pressure from the endoscopist and intrinsic force from the endoscope - influence the endoscope images by causing unpredictable motion. This motion in turn influences the baseline situation, which makes the main assumption false at times (Figures 2.3 and 2.4).

For an accurate result, the previous direction and movement need to be taken into account, just as the environment and information about tip orientation. These data may help estimate where the lumen center is located, even if it is not in the field of view. The endoscope can then be steered with this lumen center location 'in mind'.

Depth information about the environment would partly solve the lack of information in dark region segmentation. However, specular reflections, caused by reflecting properties of the mucosa (Figure 2.5), have a high pixel intensity. Depth calculations will therefore result in apparent reflections that are extremely close to the camera, while their real location lies on the organ wall. A solution to this problem is to apply preprocessing steps on the images, such as filtering $[40,45]$, but the exact tip orientation 


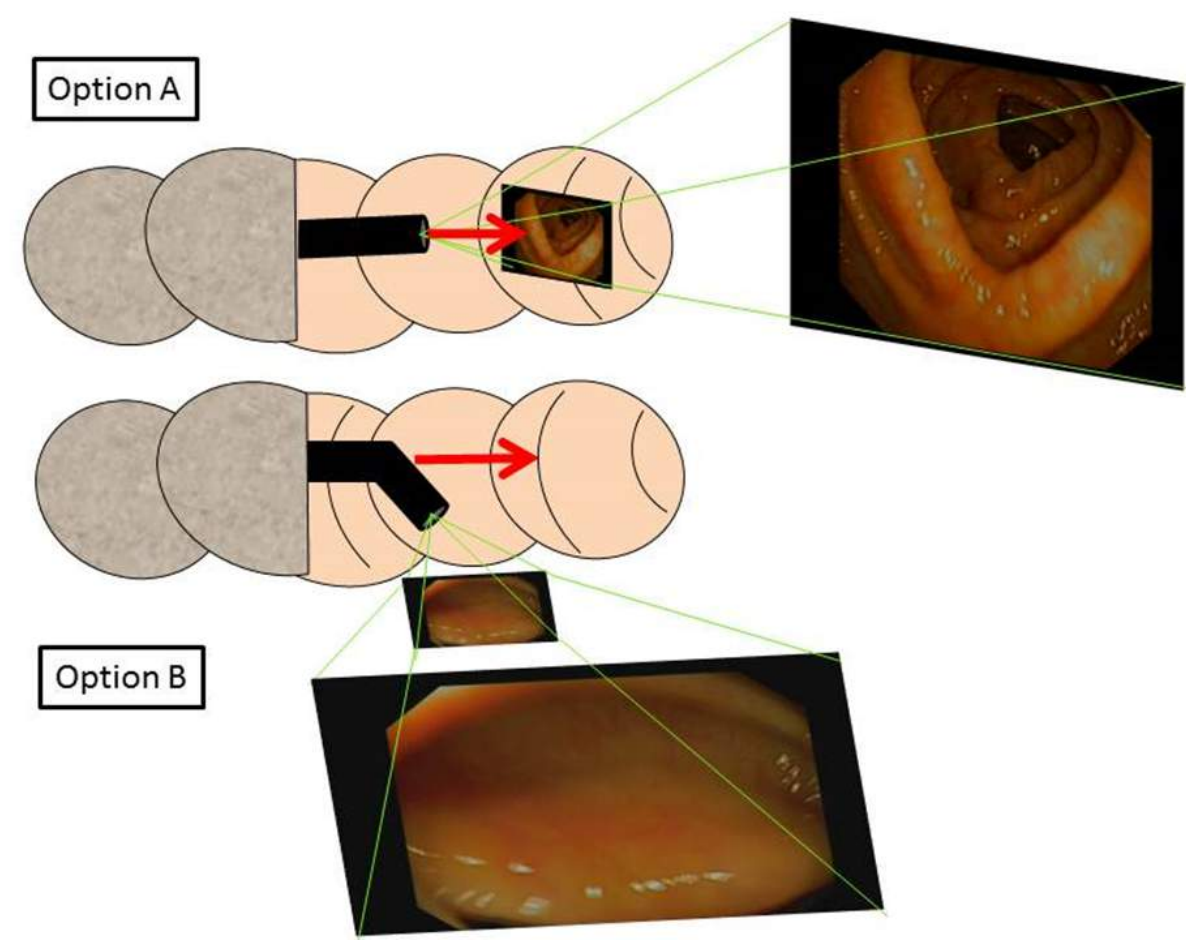

Figure 2.4: Schematic representation of flexible endoscopes in the colon. Two possible situations may occur $(\mathbf{A}, \mathbf{B})$ during which no steering motion is required. In lumen centralization techniques, no steering will take place in option $\mathbf{A}$, but a correctional steering motion will be initiated in option $\mathbf{B}$

remains unclear.

Shape from shading technology theoretically provides information about the environment, and possibly about tip orientation. Previous endoscope motion can be derived from this, which makes this an apparent ideal technique for visual endoscope navigation. However, the main problem in shape from shading technology is computer calculation time [42]. Additionally, some surfaces reflect the light unpredictably, specifically mucosa-covered surfaces, due to the presence of mucus and fluids [28]. This will make inverting the reflectance equation highly unpredictable.

\section{Visual odometry}

Visual odometry comprises the technique of (automatically) obtaining landmarks, or unique recognition points, and tracking them throughout an image sequence with the aim to derive position and orientation information. These landmarks, such as vascular junctions in the organ wall, are automatically found by the software. 'Optical flow' is the technique that calculates the displacement of these landmarks between two im- 

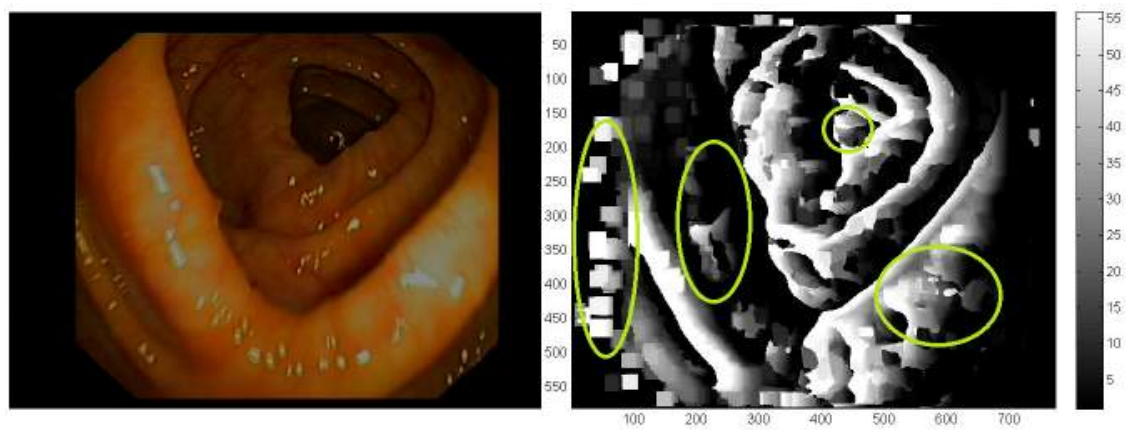

Figure 2.5: Example of depth profile from two images: left original (first) image, right depth image. It can be observed that boundaries and reflecting surfaces cause difficulty in depth estimation (ellipses)

ages and uses these displacements to calculate endoscope movement. The landmark displacements can be depicted as a field with arrows, 'vectors', indicating the direction and the length of the shift. Such a field is then called an 'optical flow field' (Figure 2.6).

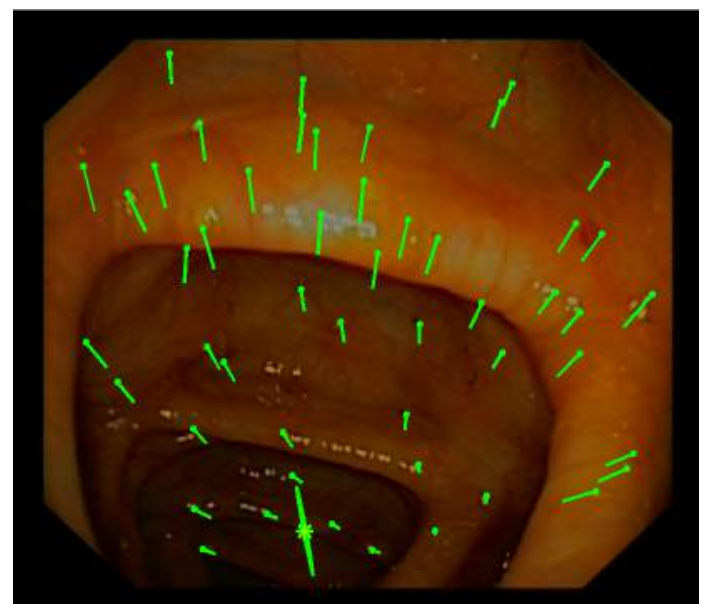

Figure 2.6: Optical flow field (with outliers). Often it is assumed that large arrows indicate nearby objects. As shown, the deeper part of the lumen displays large arrows as well and nearby parts display small arrows. Therefore, the size assumption will in this case lead to a lack in robustness of the steering algorithm.

The optical flow can be calculated in different manners, but in this paper, all different kinds of optical flow methodologies are referred to as 'optical flow' or 'optical flow calculation'. In many applications, optical flow is used to find the point from which 
all displacement vectors in the optical flow field seem to emerge, the so-called Focus of Expansion (FOE). This FOE corresponds to the current heading direction of the endoscope and can be obtained in any environment, as long as there are detectable displacements.

Mostly, both detection and tracking of landmarks are combined in one application. Between 1980 and 2011 many different ways to do this have been proposed. Previous research was done on applications in human colonoscopy images [45,46], in which was concluded that automated endoscope navigation was feasible. Tests of optical flow algorithms can be performed on simulated images, rendered by computers. Reilink et al. [32] roughly modeled the colon and used an optical flow algorithm to steer the tip of the endoscope towards the lumen center. They also implemented a steering algorithm and tested it on colon images from an endoscopy simulator (Accutouch, CAE healthcare, Montreal, Quebec, Canada) [33]. In the paper of Masson et al. [47], three different tracking algorithms are compared in a simulated setting. Deguchi et al. [48] discuss a technique to calculate the 3D shape reconstruction from endoscopic images. This method is called the Factorization Method [49], and is based on the assumption that the observed object remains constant, while the camera motion causes a detectable optical flow pattern. Points far away produce a displacement pattern with small vectors, while nearby points cause large vectors.

Deguchi, Mori and others [50] have extended the visual odometry technique by comparing bronchoscopy images to pre-procedural CT images. This way, the endoscopist not only is able to see the inside of the lungs, but also the surrounding anatomy and the exact current location of the endoscope. Note that bronchi form a relatively stable environment, which makes prior knowledge reliable. Their result seems promising and solves some of the problems that are encountered when using optical flow.

Disadvantages of visual odometry In visual odometry the same forces, mentioned in the 'Disadvantages of lumen centralization' section, still cause problems in image interpretation. However, a combination of knowledge about the traveled path and the environment may predict adequate progression of the endoscope, even if the images are not suitable for highly accurate analyses. This information can be obtained from reliable optical flow calculations, which means the right optical flow algorithm needs to be applied. To date, no systematic comparison of optical flow algorithms on human endoscopic images has been done to our knowledge.

Optical flow algorithms furthermore are very sensitive to illumination changes [47], motion blur and fluid artifacts, and computational time could be problematic in some algorithms. Specular reflections will have to be filtered out to diminish illumination changes. However, these reflections will only lead to falsely tracked landmarks in the neighborhood, which does not necessarily mean that the complete image is tracked falsely. Optical flow has the advantage of orientation information through tracking endoscope progression, which makes a predictive algorithm theoretically possible. 


\subsection{Discussion}

Aim of this paper is to describe the most promising approaches for flexible endoscope navigation from currently known research. Two fields of interest are identified: lumen centralization and visual odometry. These approaches have not led to commercially available steering systems yet. This is mainly due to vital technological problems that have not been solved.

Both approaches mostly face robustness issues, and computational time sometimes leads to a non-real-time solution. Other challenges exist; predictive organ models are hard to design due to highly deformable organs. In lumen centralization techniques, the lumen cannot be discerned in all images. Most lumen centralization techniques are very sensitive to illumination changes at the organ surfaces, more than optical flow algorithms. Other problems include image distortion due to residual organ or rinsing fluids. A last challenge is caused by the assumption in most research that organs are rigid tubes. Since most organs are in fact non-rigid, highly deformable tissues that collapse without internal counter-forces from air or fluids, this assumption leads to a lack of robustness in methods for image-based estimation of tip orientation (like contour extraction). Additionally, flaws in underlying navigational models for steering algorithms arise, since steering inside tubes is not similar to steering inside organs.

Hardware tools are needed to implement navigation algorithms on a flexible endoscope system. These tools will probably result in additional costs for hospitals. Therefore, accessory hardware tools to facilitate software solutions are generally considered a disadvantage. The upside of the accessory tools is that such systems hold potential for performing more complex procedures [11]. Adjusting the hardware of a system or building a completely new one may lead to another kind of automated flexible endoscopy system [51, 52]. However, the currently available systems are not ideal $[11,10]$. Yeung et al. [11] discuss all currently available flexible endoscopic multitasking platforms. With 'available' is meant: at least in an advanced prototype state. Multitasking platforms are specifically designed to perform highly complex intraluminal interventions or even complete NOTES procedures. These platforms tend to be technologically complex and most solutions require a complete new endoscope design, such as the Anubis endoscope (Karl Storz GmbH \& Co. KG, Tuttlingen, Germany) and the EndoSamurai (Olympus Corp., Tokyo, Japan). Of the discussed platforms, only two use conventional flexible endoscopes, which is suggested to be an easier and cheaper option. These platforms are the Direct Drive Endoscopic System (DDES, Boston Scientific, Natick, MA, USA) and the Incisionless Operating Platform (IOP, USGI Inc., San Clemente, CA, USA). Flexible endoscopy systems could theoretically even be extended to complete telemanipulation platforms, comparable to the Da Vinci surgical system (Intuitive Surgical, Inc., Sunnyvale, CA, USA). In telemanipulation platforms, the mechatronics that control the instruments are controlled by a computer. This computer can be programmed to add functionality to the instruments, such as automated navigation.

Despite the challenges mentioned, automating endoscope steering has potential. Automation of endoscope steering may lead to an easier endoscope introduction and consequently increase procedure efficiency. It holds potential to lower the costs of diagnostic and small therapeutic intraluminal procedures, and to increase patient safety. 
At the University of Twente a telemanipulation system for flexible endoscopes is being developed [46,53]. The primary goal is to develop a cockpit-like surgical platform for complex intraluminal interventions and improve current diagnostics and therapies. The idea behind the design is to make a low-cost, complete system that is suitable for current clinical practice. For most hardware, the proof-of-principle level is achieved. Additionally, software is being developed for intuitive insertion of the endoscope. Improvements in the steering model are realized by assuming a completely unpredictable environment - and not a rigid tube. A target lock, automatic loop detection and realtime positional feedback are among the future functionality of the system.

Additionally, therapeutic interventions could benefit from automated steering. Mori et al. [54] aim to real-time track the camera motion of a bronchoscope by utilizing preprocedural data in the form of CT images. This information can then be employed as a roadmap for an endoscopic navigation system. Performing procedures inside the bronchi and through the bronchial wall becomes easier, since exact localization of relevant lymph nodes and the point of interest is possible. Their method could possibly be extended to other organs, such as the colon.

Some advanced surgical procedures, like the peroral Heller myotomies (POEM), are already conducted with flexible endoscopes. To expand this field further, automated systems can be of advantage. Automated position control, improved visualization by image processing and an ergonomic user interface instead of steering knobs are all possibilities of telemanipulated flexible endoscopy systems. If these functionalities are indeed realized, surgical procedures through flexible endoscopes may form a superior method of minimally invasive surgery.

In conclusion, vision-based navigation for endoscope steering is widely investigated and likely to enter the clinic not far from now. The implementation of automated flexible endoscope steering possibly holds major advantages for physicians, patients, and extension of the flexible endoscopy field in general. Research is currently focused on developing low-cost hardware solutions and technologically robust steering algorithms, in which real-time implementation of visual navigation techniques will play a major role. Clinical applications are theoretically numerous and the first ones are expected within five years.

\subsection{Appendix}

Table 2.1: Content overview of the included papers

\begin{tabular}{lll}
$\begin{array}{l}\text { Paper } \\
\text { (first author, year) }\end{array}$ & Main inclusion detail(s) & Additional remarks \\
\hline Gillies, 1996 & $\begin{array}{l}\text { Development and testing } \\
\text { of an automated flexible } \\
\text { endoscopy steering algo- } \\
\text { rithm }\end{array}$ & $\begin{array}{l}\text { Based on lumen central- } \\
\text { Kwoh, } 1999\end{array}$ \\
$\begin{array}{l}\text { Additional techniques for } \\
\text { the platform described in } \\
\text { Gillies, } 1996\end{array}$ & Based on lumen central- \\
&
\end{tabular}


Table 2.1: Content overview of the included papers

\begin{tabular}{|c|c|c|}
\hline $\begin{array}{l}\text { Paper } \\
\text { (first author, year) }\end{array}$ & Main inclusion detail(s) & Additional remarks \\
\hline Chettaoui, 2006 & $\begin{array}{l}\text { Extraction and tracking } \\
\text { of the central lumen of } \\
\text { the colon from flexible } \\
\text { endoscopy images }\end{array}$ & $\begin{array}{l}\text { Lumen segmentation } \\
\text { technique }\end{array}$ \\
\hline Bricault, 1998 & $\begin{array}{l}\text { Registration of preopera- } \\
\text { tive CT scans to facilitate } \\
\text { computer-assisted trans- } \\
\text { bronchial biopsies: an } \\
\text { image-guided endoscope } \\
\text { application }\end{array}$ & $\begin{array}{l}\text { Lumen detection by } \\
\text { shape from shading } \\
\text { technology }\end{array}$ \\
\hline Reilink, 2010, 2011 & $\begin{array}{l}\text { In vitro testing of auto- } \\
\text { mated flexible endoscope } \\
\text { steering technologies }\end{array}$ & $\begin{array}{l}\text { Lumen centralization } \\
\text { based on center of grav- } \\
\text { ity detection and optical } \\
\text { flow analysis }\end{array}$ \\
\hline Zhiyun, 2000 & $\begin{array}{l}\text { Multiple image-guided en- } \\
\text { doscope applications us- } \\
\text { ing esophageal images }\end{array}$ & $\begin{array}{l}\text { Lumen segmentation } \\
\text { technique }\end{array}$ \\
\hline Zhen, 2006 & $\begin{array}{l}\text { Fully automated flexible } \\
\text { endoscopy platform; not } \\
\text { clinically tested }\end{array}$ & $\begin{array}{l}\text { Lumen segmentation by } \\
\text { auto-thresholding }\end{array}$ \\
\hline Krishnan, 1994 & $\begin{array}{l}\text { Lumen detection for } \\
\text { image-guided endoscopic } \\
\text { applications }\end{array}$ & $\begin{array}{l}\text { Lumen segmentation by } \\
\text { boundary extraction }\end{array}$ \\
\hline Tjoa, 2003 & $\begin{array}{llr}\text { Lumen detection for } \\
\text { clinical diagnosis in } \\
\text { colonoscopy }\end{array}$ & $\begin{array}{l}\text { Lumen segmentation by } \\
\text { boundary extraction }\end{array}$ \\
\hline Xia, 2003 & $\begin{array}{l}\text { Lumen detection for } \\
\text { computer-assisted diag- } \\
\text { nosis in colonoscopy }\end{array}$ & $\begin{array}{l}\text { Lumen extraction by } \\
\text { boundary detection }\end{array}$ \\
\hline Batlle, 1998 & $\begin{array}{l}\text { Review and applications } \\
\text { in coded structured light } \\
\text { with possible applications } \\
\text { in depth analysis in im- } \\
\text { ages }\end{array}$ & $\begin{array}{l}\text { No explicit application in } \\
\text { flexible endoscopy, but a } \\
\text { comprehensive overview } \\
\text { of the technique }\end{array}$ \\
\hline Salvi, 2004 & $\begin{array}{l}\text { Pattern codification for } \\
\text { structured light }\end{array}$ & $\begin{array}{l}\text { No explicit application in } \\
\text { flexible endoscopy, but } \\
\text { an overview of the tech- } \\
\text { nology }\end{array}$ \\
\hline
\end{tabular}


Table 2.1: Content overview of the included papers

\begin{tabular}{|c|c|c|}
\hline $\begin{array}{l}\text { Paper } \\
\text { (first author, year) }\end{array}$ & Main inclusion detail(s) & Additional remarks \\
\hline Zhang, 2005 & $\begin{array}{l}\text { Application of structured } \\
\text { light for surface recon- } \\
\text { struction in industrial flexi- } \\
\text { ble endoscopy }\end{array}$ & $\begin{array}{l}\text { Surface reconstruction } \\
\text { in flexible endoscopy } \\
\text { (depth estimation) }\end{array}$ \\
\hline Mekaouar, 2009 & $\begin{array}{l}3 D \text { reconstruction from } \\
\text { the inside of the colon } \\
\text { from } 2 D \text { images, used in } \\
\text { a motorized colonoscopy } \\
\text { system }\end{array}$ & $\begin{array}{l}\text { Surface reconstruction } \\
\text { using shape from shad- } \\
\text { ing technology (depth } \\
\text { estimation) }\end{array}$ \\
\hline Ciuti, 2012 & $\begin{array}{l}\text { Depth estimation with } \\
\text { shape from shading tech- } \\
\text { nology to finally be applied } \\
\text { in an endoscopic active } \\
\text { locomotion platform }\end{array}$ & $\begin{array}{l}\text { Surface reconstruction } \\
\text { using shape from shad- } \\
\text { ing technology (depth } \\
\text { estimation) }\end{array}$ \\
\hline Rashid, 1992 & $\begin{array}{l}\text { Original shape from shad- } \\
\text { ing explanation, develop- } \\
\text { ment and testing }\end{array}$ & $\begin{array}{l}\text { No explicit application in } \\
\text { flexible endoscopy, but } \\
\text { an overview of the tech- } \\
\text { nology }\end{array}$ \\
\hline $\begin{array}{l}\text { Van der Stap, } \\
\text { 2012a, -b }\end{array}$ & $\begin{array}{l}\text { The use of optical flow as } \\
\text { a navigation clue for flex- } \\
\text { ible endoscope automa- } \\
\text { tion }\end{array}$ & $\begin{array}{l}\text { Visual odometry in flexi- } \\
\text { ble endoscopy }\end{array}$ \\
\hline Masson, 2009 & $\begin{array}{l}\text { Comparison of three } \\
\text { tracking algorithms for } \\
\text { interventional flexible } \\
\text { endoscopy }\end{array}$ & $\begin{array}{l}\text { Visual odometry in flexi- } \\
\text { ble endoscopy }\end{array}$ \\
\hline Deguchi, 1994 & $\begin{array}{l}\text { 3D surface reconstruction } \\
\text { from endoscopic images } \\
\text { using the factorization } \\
\text { method }\end{array}$ & $\begin{array}{l}\text { Visual odometry in flexi- } \\
\text { ble endoscopy }\end{array}$ \\
\hline Poelman, 1997 & $\begin{array}{l}\text { Original posing of the } \\
\text { idea for the factorization } \\
\text { method }\end{array}$ & $\begin{array}{l}\text { No explicit application in } \\
\text { flexible endoscopy, but } \\
\text { an overview of the tech- } \\
\text { nology }\end{array}$ \\
\hline Deguchi, 2003 & $\begin{array}{l}\text { Motion tracking in flexible } \\
\text { endoscopy navigation }\end{array}$ & $\begin{array}{l}\text { Visual odometry in flexi- } \\
\text { ble endoscopy }\end{array}$ \\
\hline
\end{tabular}




\subsection{References}

Please refer to the Bibliography at the end of this thesis. 
CHAPTER 3

\section{The focus of expansion in automated flexible endoscope steering}

Lewis Carroll

Published as: N. van der Stap, R. Reilink, S. Misra, I.A.M.J. Broeders, \& F. van der Heijden (2012). The Use of the Focus of Expansion for Automated Steering of Flexible Endoscopes. In: 4th IEEE RAS \& EMBS Int. BioRob Conf., 24-27 June, 2012, Rome, Italy. pp. 13-18. ISSN 2155-1774. ISBN 978-1-4577-1199-2.

Copyright (C) 2012 IEEE

The images captured by the camera at the endoscope tip are used to find the heading direction of the endoscope. Comparing the current heading direction to the desired target direction in a computer algorithm is expected to allow automated steering of the endoscope. Heading direction determination is achieved using an estimation of the Focus of Expansion (FOE) from an optical flow field. The resulting heading direction is compared to results obtained manually by several human observers. From these experiments it becomes clear that the FOE can be used as a reliable estimator for heading direction in human colonoscopy images. 


\subsection{Introduction}

Flexible endoscopy can be performed to obtain a diagnosis and to perform small interventions in the human body without leaving scars. In screening for colorectal cancer [55], flexible endoscopy of the large bowel (colonoscopy) is performed to diagnose and, if necessary, remove lesions of the bowel wall. It is expected that the number of colonoscopies performed yearly in the Netherlands will increase by 60.000 after the start of the population screening program in 2013 [13]. This rise motivates hospitals to increase their colonoscopy capacity and efficiency drastically.

Currently, an endoscope driver (endoscopist) needs to perform a large number of procedures to reach the level of competence. Estimates of this number vary from 100 to 500 colonoscopic procedures over periods of 1 to 3 years $[17,56,57]$. Shortening the learning curve by facilitating the procedure would be one way to increase efficiency in colonoscopy departments. A second approach could be to have less-trained personnel perform the routine procedures, while leaving the more complicated ones to more highly trained gastroenterologists or surgeons. Finally, the introduction time of colonoscopes could be reduced to increase productivity. This research is aimed at a simplification of the steering mechanism of flexible endoscopes in order to bring about efficiency increases.

\subsubsection{The endoscope}

A flexible endoscope consists of a hand grip attached to a flexible tube with a camera, an illuminator, and one or more working channels $[19,58]$. The camera consists of a lens and a charged coupled device (CCD) or complementary metal oxide semiconductor (CMOS) chip. Manual control of flexible endoscopes is considered impractical and unintuitive [24]. The endoscopist folds one of his or her hands around the hand grip in order to steer the instrument. Two steering knobs on the hand piece can be turned to gradually bend the tip in the directions 2 and 3 (Figure 3.1). The tip of the endoscope has four degrees of freedom: longitudinal translation and rotation (represented by arrows 1 and 4), and two combined rotations/translations (arrows 2 and 3). A predominantly bimanual steering method frequently results from the limiting design of the device, because most hands are too small for single-handed steering. However, bimanual steering does not allow simultaneous insertion or retraction of the endoscope by the same person.

The impractical way of steering has motivated scientists to consider automated flexible endoscope steering. Gillies et al. [28] tried steering a flexible endoscope automatically using computer vision. Edge detection and the wall contour shape of the bowel were used to estimate depth and steer the endoscope. Although the steering itself was successful, the interpretation of images of the wall remained problematic. Also, many hardware adjustments were needed.

Reilink et al. [32] investigated image-guided flexible endoscope steering as well. They used an 'image intensity'-based method and an 'optical flow'-based method to steer the endoscope tip towards the center of the lumen (the cavity enclosed by the walls of the bowel). Optical flow (OF) refers to the vectors that represent two-dimensional 

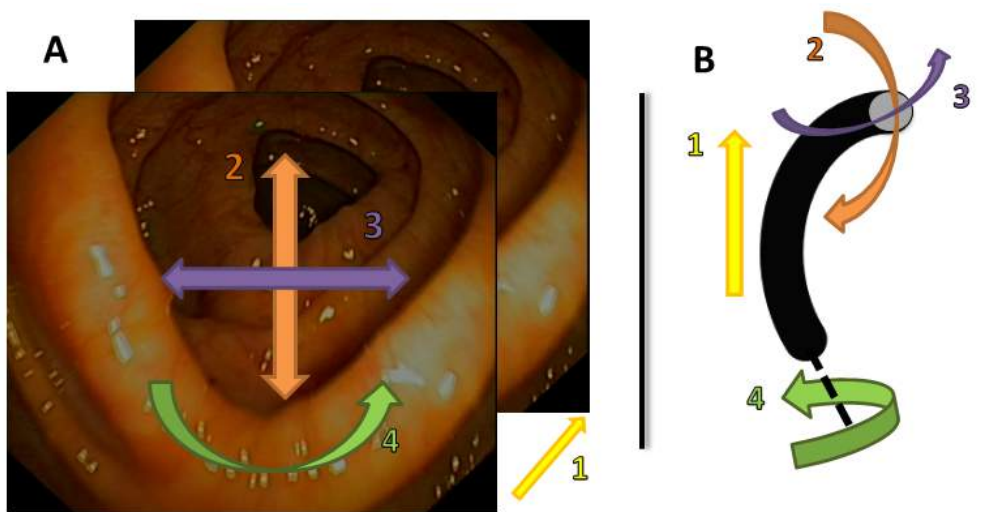

Figure 3.1: A) consecutive images of the human bowel. B) schematic endoscope. Possible degrees of freedom are depicted and numbered in both. The endoscope is inserted in direction 1. It can be rotated along the shaft (direction 4), and the tip can bend up/down (direction 2) and left/right (direction 3). Copyright (C) 2012 IEEE

(2D) displacements of (groups of) pixels in two consecutive images in the image plane. These pixel displacements are caused by the three-dimensional (3D) motion of scene and camera. If the camera is shifted in the longitudinal direction of the tube, objects that are far away will show small or even zero displacements in the image plane. Objects in a more lateral position or nearby will have larger displacements. Theoretically, steering away from the larger displacements will lead the endoscope towards the most distant point in space. The 'image intensity' algorithm proposed by Reilink et al. [32] is based on different areas of brightness in the image. The colon lumen is assumed to correspond to a dark area in the image. Steering the endoscope by keeping this dark area in the center of the image will lead to a centered path through the colon. Reilink et al. [10] conducted experiments on both simulated images and images from the inside of an anatomical model. The conclusion was that automatically steering a flexible endoscope was feasible, but more work was needed to design a robust system.

Other research on automated flexible endoscope steering focused mainly on lumen centralization of the tip. In [30, 47, 34], a variety of methods for lumen detection are proposed.

\subsubsection{The images}

The images produced by a colonoscope camera viewing the inside of the human colon (real images) are more difficult to use for automated steering than the images used by Reilink et al. [32]. Real images often suffer from motion blur, out-of-focus artifacts and even less texture on wall surfaces than in the anatomical model. Furthermore, in many images the lumen is not located in the field of view (Figure 3.2), so it is not enough to assume that centralization of the darkest region is enough to steer the colonoscope. 


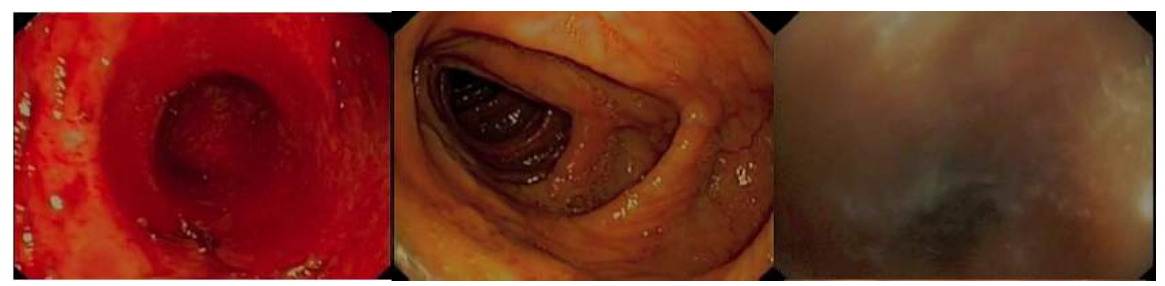

Figure 3.2: Examples of human colonoscopy images. The first two images consist of a lumen view of very different human colons. The third image (right) is an example of a blurred image, caused by irrigation to clean the lens. Copyright (c) 2012 IEEE

Although the objective of Mori et al. [59] slightly differed from ours, their approach was also to use optical flow. The detected features and displacement information were combined to reconstruct a 3D replica of the environment of the camera without the need for a lumen view. This led to the conclusion that a combination of dark region detection (DRD) with OF techniques might provide the necessary information to steer the endoscope automatically. However, the assumption that large displacements indicate nearby objects (Reilink et al., red.) is based on the premise that the tip only moves in the longitudinal direction in a corridor-like environment. Yet in flexible endoscopy, the tip movement and the environment are more complex (Figure 3.1). Optical flow displacements form a pattern of vectors that radially emerge from or point to a specific point, depending on the direction of movement. This point is called the focus of expansion (FOE) [60]. The distance of a displacement vector to this FOE also influences vector size, especially when sideward and forward motions are combined (Figure 3.3). This fact is not discussed in the other work on flexible endoscope navigation.

\subsubsection{Contribution and paper outline}

The current research is designed around the same driving unit that Reilink et al. [32] describe. The aim of this research is to develop a suitable computer vision algorithm to apply to human colonoscopy images. This algorithm can then be used to automatically steer flexible endoscopes. Two research objectives are defined in this paper. Firstly, OF and the FOE estimation are implemented in a mathematical model. Secondly, we test whether calculation of the OF and a corresponding FOE in human colonoscopic images is feasible.

In section 3.2 of this paper, both relevant terms and the theory that we developed are discussed. In section 3.3 we present an experimental set-up for evaluating FOE estimation in flexible endoscopy. The results in terms of the found accuracy of the FOE and its use in navigation will be given in section 3.4. Section 3.5 discusses these results, while in section 3.6 the conclusions of this paper are presented. 

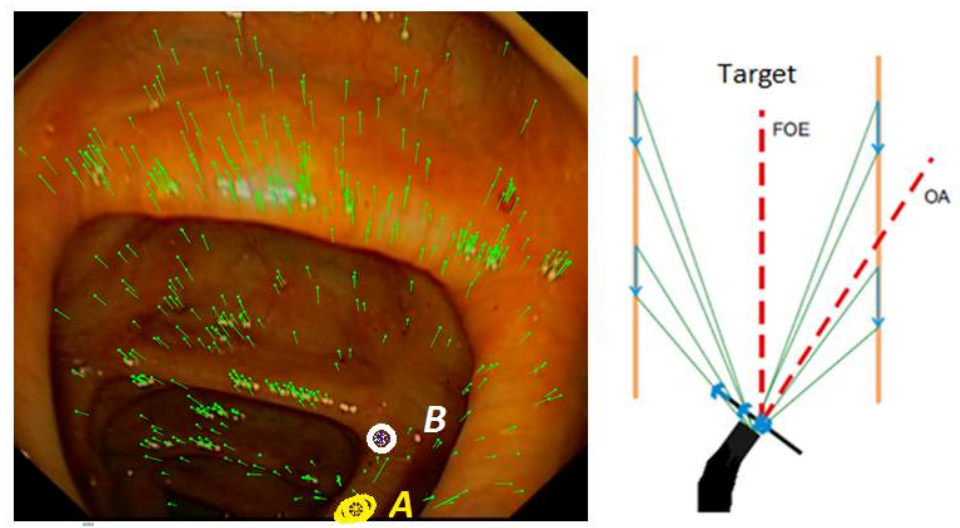

Figure 3.3: Example of calculated FOE (A) and manual FOE (B) within OF field in real image (left). On the right, a schematic figure of OF arrow origin (arrows) and FOE direction vs. target and tip direction $(\mathrm{OA})$ is depicted. Note that the target direction is not always along the optical

axis, which makes steering superfluous in this example case. Copyright (C) 2012 IEEE

\subsection{Endoscope navigation}

At least three parameters are needed to steer an endoscope (Figure 3.3): the direction in which the endoscope is currently heading (heading direction), the direction of the target (target direction) and the direction in which the tip is pointing (tip direction or Optical Axis (OA) as shown in Figure 3.3). These are all directions in 3D space, but they can conveniently be represented as 2D positions in the image plane.

It is known that the FOE (Figure 3.3) corresponds to the heading direction for translational movements [60], provided that the effect of angular velocities of the tip is negligible. We need to assess whether the FOE holds as a measure for heading direction in our case. Section 3.2.3 discusses this aspect in more detail.

The target direction is derived from the darkest region in the image. This assumes no other dark region exists. We observed that the lumen is not visible in all images. However, for now we assume that the darkest region is the most distant point in the colon, which subsequently makes it the target. In the case of the disease diverticulosis, bulges exist in the bowel wall that can be mistaken for the colon lumen when observed from the inside. At the moment, our algorithm has no way of coping with this situation, but a possible solution is proposed in Chettaoui et al. [30].

The tip direction corresponds to the optical axis and can be determined continuously by measuring steering knob position, as is performed by the driving unit. The control algorithm will be focused on keeping the FOE near the darkest region by correcting the tip direction when needed. This approach differs from other approaches because it prevents correctional steering in the situation where the endoscope is heading towards the lumen as a whole but the tip is pointing in another direction (Figure 3.3, right). 
The following three subsections accomplish the first research objective which was to explore the feasibility of using the FOE in a navigational model for flexible endoscopy. Firstly, subsection 3.2.1 describes the dark region detection in more detail. Subsection 3.2.2 then describes the calculation of the OF field and finally, in subsection 3.2.3, FOE estimation is discussed.

\subsubsection{Dark region detection}

Finding the lumen in an image is performed using DRD. The dark region is found recursively by searching the minimum of the low-pass-filtered intensity (gray value) image in an area around the previously found location. Before the algorithm can start, the lumen is indicated manually in the first image.

\subsubsection{Calculating optical flow}

Calculation of OF is commonly based on a block motion model. The common assumptions are that a) the OF within a small block (window) in the image plane is constant; and that b) the pixels within corresponding blocks in consecutive images do not change. The latter is often referred to as the constant brightness assumption. These assumptions are used in, for instance, the Lucas-Kanade tracker [61], but do not hold well in human endoscopy images. The Horn-Schunck method is based on the assumption that the OF vectors only change smoothly along the image plane [62]. This assumption also does not hold in human colon images, especially not in the vicinity of the FOE. Therefore, a different method of OF calculation is needed. Another problem comes in when trying to perform adequate feature detection in these images. Lack of texture and specular reflections cause problems detecting enough features with high reliability. As a result, the risk of mismatches is high.

We have searched for an existing technique that can detect blob-like features and at the same time has some kind of match verification between those features. The scale invariant feature transform (SIFT) point feature detector [63] fulfills both requirements. A set of candidate 2D image points to track is selected in each of two consecutive images. Matching is based on feature vectors that are extracted from the neighborhood of the detected points. The features of the current frame are matched to those of the previous frame and a displacement vector between them can then be determined. These vectors can be regarded as alternative OF vectors, and together they form the OF field.

\subsubsection{Estimating the focus of expansion}

Estimation of the FOE, denoted by $\mathbf{q}$, is achieved by means of weighed Least Square Estimation (LSE). For that, we need a linear observation model of each pair of matched points:

$$
\mathbf{z}=\mathbf{M q}+n
$$

where $\mathbf{z}$ is an observation, $\mathbf{M}$ is the measurement matrix, and $n$ is the measurement noise. Such a linear model is based on the fact that the FOE is found on the line that connects the two matching points. This is true in the case of a translational motion and 
a static scene. Thus, if $\mathbf{p}_{\mathbf{1}}$ is a point found in the first image, and $\mathbf{p}_{2}$ is the corresponding matching point in the second image, then $\mathbf{q}, \mathbf{p}_{1}$ and $\mathbf{p}_{2}$ are collinear. If $\mathbf{q}=\left[x_{q} y_{q}\right]^{T}$ then:

$$
z=a x_{q}+b y_{q} \text { for } a^{2}+b^{2}=1 .
$$

in which $z, a$, and $b$ follow from $\mathbf{p}_{1}$ and $\mathbf{p}_{2}$ using their collinearity. The definition of $\mathbf{M}=[a b]$ then results in eq. 3.1. The noise $n$ originates from the uncertainty of the point locations. The errors in the locations propagate through eq. 3.2 to $n$. eq. 3.2 provides us with the model for a single pair of matching points, but in reality there are $N$ pairs of matching points leading to $N$ equations resembling eq. 3.2. These equations can be stacked to yield:

$$
\mathbf{z}=\mathbf{H q}+\mathbf{n},
$$

where $\mathbf{H}$ is the $N$ times stacked version of $\mathbf{M}$ from eq. 3.2.

The noise vector $\mathbf{n}$ is quantified by its covariance matrix $\mathbf{C}_{\mathbf{n}}$. The matrix will be diagonal, assuming that errors in the point locations are independent. From the geometry of the problem (radial expansion originating in $\mathbf{q}$ ) we assume that the standard deviation of $\mathbf{n}$ is inversely proportional to the distance $d_{n}$ between $\mathbf{p}_{1}$ and $\mathbf{p}_{2}$, and proportional to the distance $D_{n}$ from the key point to $\mathbf{q}$. The covariance matrix then is described by:

$$
\mathrm{C}_{\mathbf{n}}=\operatorname{diag}\left(\sigma_{n}^{2} \frac{D_{n}^{2}}{d_{n}^{2}}\right)
$$

If we assume equal uncertainties for all measurements, and no prior knowledge on q, the Least Square Estimator $\hat{\mathbf{q}}_{\text {LSE }}(\mathrm{LSE},[64])$ is:

$$
\hat{\mathbf{q}}_{L S E}=\left(\mathbf{H}^{\mathrm{T}} \mathbf{H}\right)^{-1} \mathbf{H}^{T} \mathbf{z}
$$

If the uncertainties are unequal, according to eq. 3.4 the maximum likelihood estimator (MLE) $\hat{\mathbf{q}}_{\mathrm{MLE}}$ becomes:

$$
\hat{\mathbf{q}}_{\mathrm{MLE}}=\left(\mathrm{H}^{\mathrm{T}} \mathrm{C}_{\mathrm{n}}^{-1} \mathbf{H}\right)^{-1} \mathbf{H}^{T} \mathbf{C}_{\mathrm{n}}^{-1} \mathbf{z}
$$

which can be regarded as an LSE in which each measurement is weighed inversely proportional to its uncertainty. Note that the weighed LSE estimator cannot be used directly since knowledge of $\mathbf{q}$ is required to calculate $D_{n}$. This leads to an iterative approach in which first an estimate is obtained using eq. 3.5, of which the result then is used in eq. 3.4 to obtain an estimate that fits the requirements using eq. 3.6. In practice, a single pass of eq. 3.5 , followed by eq. 3.4 and eq. 3.6 suffices. In the next Section, the methods used for testing the FOE and OF field calculations are described.

\subsection{Experimental setup}

The second research objective revolves around the question: 'Is calculation of the OF and a corresponding FOE in human colonoscopy images feasible?'. We designed the experiment described below to find an answer. 


\subsubsection{Image acquisition}

The images used were acquired during human colonoscopy procedures. They were produced by a colonoscope camera (types CFQ-165 L and 160/180 AL of Olympus, Tokyo, Japan) and captured using an ADVC55 video capture device (GrassValley, San Francisco, CA, USA). Storage of images was on a laptop. From the stored movie files, a clip was selected from which completely red frames (wall views) were absent. This series was 76 frames in length.

\subsubsection{Processing}

To obtain as much information from the images as possible, preprocessing was performed. The main goal here was to find as many 2D image points to track (key points) as possible. The large black area that surrounded the image of interest, was excluded from processing by using a similarly shaped mask. The subsequent three main steps that were taken are described in the following subsections. In the flow charts, each preceding step is represented as a single block (Figures 3.4, 3.5 and 3.6).

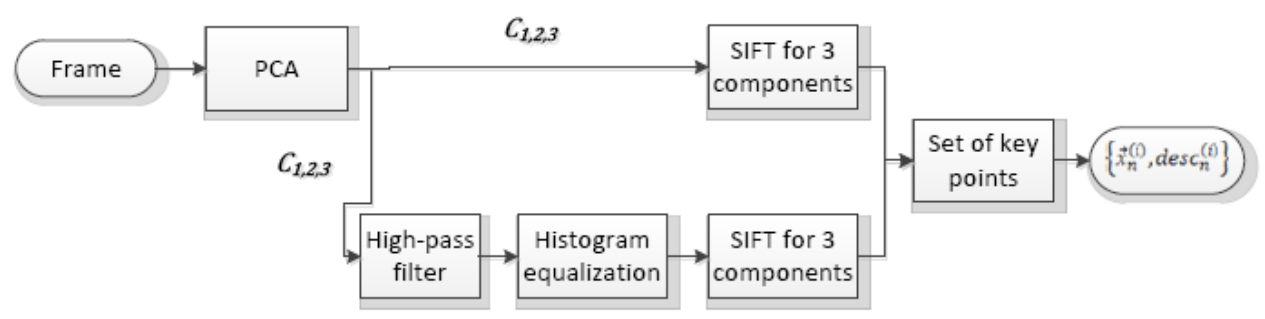

Figure 3.4: Flow chart describing steps in SIFT feature extraction. Copyright (c) 2012 IEEE

\section{SIFT key point extraction}

Two sequential color images were read. On both images, a Principal Components Analysis (PCA) [65] was performed $\left(\mathrm{C}_{1,2,3}\right)$ on the three color channels and SIFT key points were obtained. This PCA was performed, because previous (unpublished) tests showed that more key points were found when information from the three channels was obtained separately. In addition, on $\mathrm{C}_{1,2,3}$ feature enhancement in the form of highpass filtering followed by histogram equalization was applied to improve the texture even further. From the resulting three preprocessed components, the SIFT key points were obtained as well (Figure 3.4). All the key points found were pooled per image and the resulting two sets were used to find matches.

\section{SIFT key point matching}

The two sets of the sequential images frame $i$ and frame $i-1$ were compared. A match was sought using the similarity metric of the SIFT algorithm [63] (Figure 3.5). The features are $\vec{x}_{\text {number }}^{\text {frame }}$ and their properties are described by descriptor $\left\{\operatorname{desc}_{\text {number }}^{\text {frame }}\right\}$. 


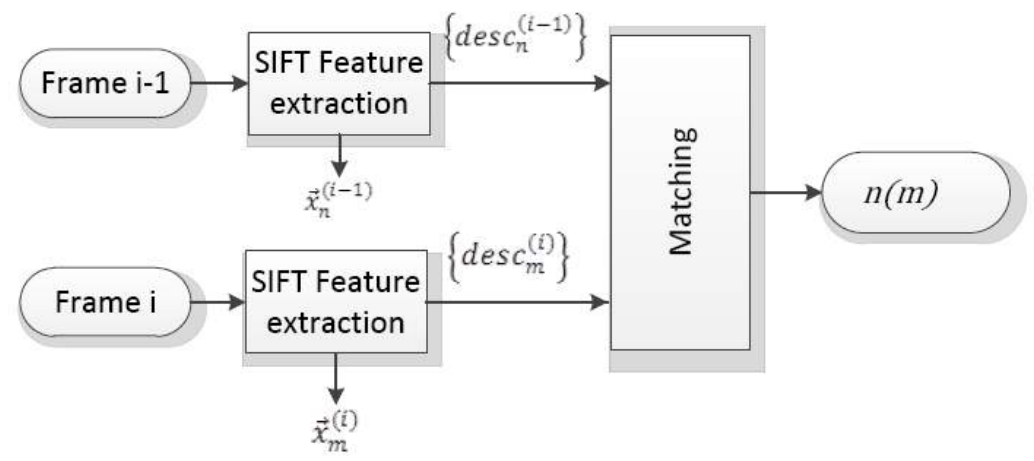

Figure 3.5: The set of SIFT key points of two consecutive frames is matched. Copyright (C) 2012 IEEE

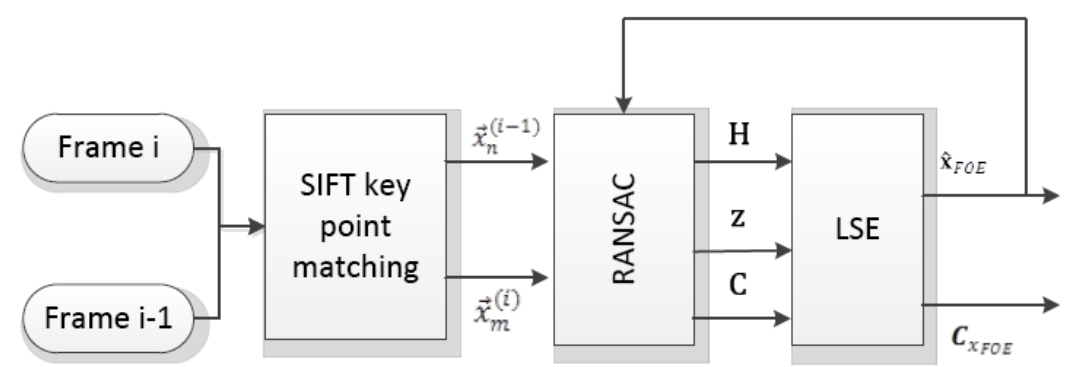

Figure 3.6: Final estimation using RANSAC and iteration. Copyright @ 2012 IEEE

This descriptor is used in the similarity metric and thus to match the key points from two different frames.

\section{Estimation}

To estimate the FOE robustly, the RANdom SAmple Consensus (RANSAC) algorithm was used [66] (Figure 3.6). This iterative algorithm estimates the FOE and accepts the estimation if enough inliers are found for the current model. Data obtained from RANSAC are the values for $\mathbf{H}, \mathbf{z}$, and the covariance matrix $\mathbf{C}$ from $\mathbf{H}$. The covariance matrix was used as a measure for the accuracy of $a$ and $b$ and for calculation of the MLE.

\subsubsection{Determining accuracy}

The automatically determined FOE location in each frame was compared to manually determined FOE locations by calculating the intraclass correlation (ICC) between the observers [67]. Independently of each other volunteers indicated the point where they 
thought the FOE was by clicking it in a window. This window iteratively showed two alternating sequential frames until a location was chosen by the volunteer.

\subsection{Experimental results}

\subsubsection{Feature detector performance}

The SIFT feature extraction algorithm was implemented according to [63]. Localization and matching of the key points was computationally intensive, and therefore analysis could not be done in real-time. Both the RANSAC and the estimation algorithm worked as expected. One of the parameters that could be set was the minimum number of inliers above which the estimation would be accepted. If RANSAC could not find a result within the acceptance threshold of this parameter, the set size was decreased. Again, this was done iteratively but only down to a minimum of $20 \%$ of the total set. If no result was found at this minimum threshold, then the frame was ignored. However, this situation did not occur during our experiments.

On average, 507 SIFT key points were matched per frame. The average number of inliers that was included by the RANSAC algorithm was 257 (50.7\%). The frame with the least found key points only showed 49 displacement vectors, 25 of which were inliers. This frame appeared to possess a lot of motion blur. The computational time for one frame was approximately 60 seconds.

\subsubsection{FOE estimator performance}

The FOE was indicated manually by three observers. When the manual information from the human observers is tested for consistency, the ICC is $88 \%$. The ICC for the observers and the automated algorithm was 89\% (Figure 3.7).

\subsection{Discussion}

SIFT key point detection is known to be a successful method of obtaining features from images that contain a lot of noise [63], and is better than, for instance, the Shi-Tomasi feature detector in finding blob-like features [68]. Human endoscopy images are not only inherently noisy, but are also lacking texture. Our preprocessing, consisting of PCA and a method resembling local histogram equalization, enhances image texture. This resulted in sufficient key points in each image which had a high enough matching rate. However, SIFT key point detection is also known for its computational cost, which is confirmed in this research as well. The algorithm currently is too computationally intensive and most of the computational time was spent in detecting and matching SIFT key points. For these operations, real-time implementations exist that use field programmable gate array (FPGA) or graphics processing unit (GPU) programming. In order to develop these, more detailed knowledge about the steering algorithm first needs to be acquired. Another, easier, approach could be to explore alternative feature detectors such as SURF [69] or FAST [70]. 

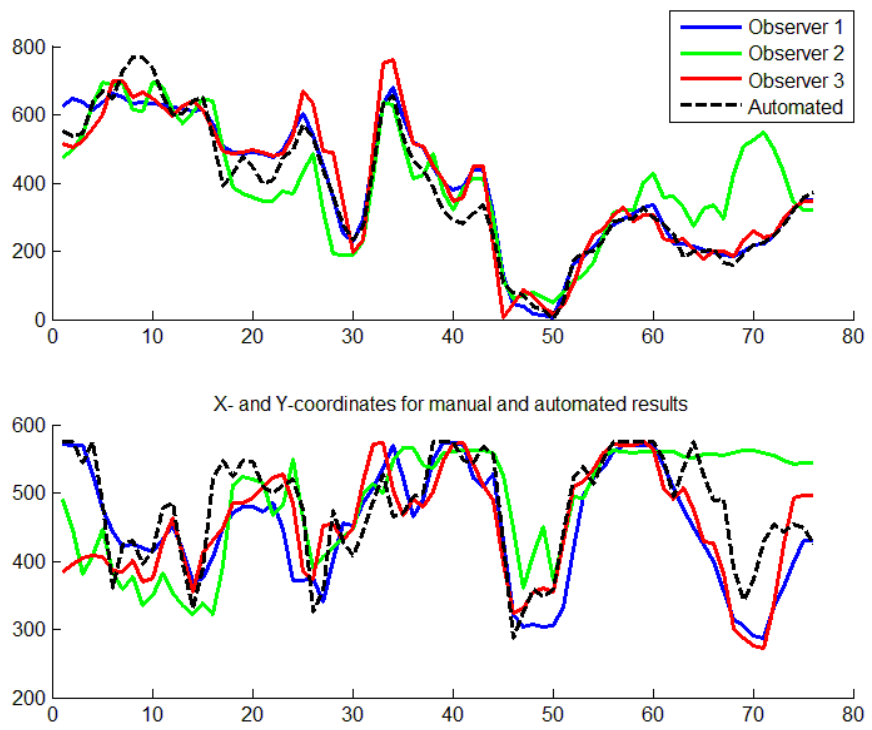

Figure 3.7: Results of manually indicated FOE by the two observers of group A and automated FOE detection plotted in one figure. The y-axis represents the pixel value of the coordinate. Copyright @ 2012 IEEE

The automated method of FOE determination has a good ICC compared to the manual FOE determination (89\%). This result indicates the high reliability of our automated FOE detection algorithm. However, we observed large differences between human observers who have a theoretical background in this area and those who do not. These differences make the ground truth less reliable, but it currently remains the best option there is.

Furthermore, results are given for a carefully selected image sequence. In human endoscopy, the tip touches the bowel wall often, which did not happen in our sequence. When there is wall contact, the produced image appears red and blurred, and key points will be hard to find. We need to either filter those images out in the final, robust, steering algorithm, or prevent wall contacts altogether.

Finally, the FOE is known to estimate heading direction in the translational direction [71]. During the experiments we also found a rotational component present in the camera movements of an endoscope. Nonetheless, rotational information can theoretically also be obtained from the images, as in the example described in [71]. 'Derotating' the image before FOE determination then becomes feasible and this in turn will make the final steering algorithm more accurate.

Several devices that could facilitate the colonoscopy procedure already exist. Most of them rely on some kind of inflatable balloon propulsion mechanism. These include the ColonoSight (Stryker Endoscopy, San Jose, CA, USA), its successor the ProtectiScope, the Aeroscope (GI-view, Ramat Gan, Israel) and the Endo-Ease system (Spirus 
Medical Inc., Stoughton, MA, USA). Caterpillar-like propulsion is applied in the Endotics system (Era Endoscopy S.r.l., Peccioli, Pi, Italy), and inverted sleeve propulsion can be found in the Invendoscope (Invendo Medical, Kissing, Germany). Although these propulsion techniques are innovative, the steering mechanisms are still mostly based on steering knobs on a hand grip that are used to control the tip. Our steering algorithm could probably benefit these devices. Additionally, the algorithm is generic and could be applied for endoscopy of other organs where navigation remains challenging. These include, but are not limited to, lungs, gall ducts and salivary glands.

\subsection{Conclusions and future work}

This study has shown that the use of the FOE for flexible endoscope navigation is feasible. The FOE can be used as a way of determining the heading direction of the endoscope mathematically, which can then be compared to a desired target direction in a steering algorithm. If these directions match, no steering movement is initiated, which keeps the number of movements to a minimum. This is the most important advantage of our approach: no unnecessary steering corrections.

Currently, it remains unknown what the influence is of the rotational component in the camera movements of the endoscope on our method. This will therefore be the subject of future work over the short term. Also, we will look into real-time improvement. Additionally, complete colonoscopy image sequences will be tested. Such sequences will consist of more images with little texture and more motion blur artifacts.

Future work will also need to determine feasibility in clinical practice. Image sequences of multiple patients will be evaluated in order to further improve the method. Over a longer term, motion estimation and the FOE will be included in a steering algorithm.

\subsection{References}

Please refer to the Bibliography at the end of this thesis. 
CHAPTER 4

\section{Comparing optical flow techniques in in-vivo images}

'The massive bulk of the earth does indeed shrink to insignificance in comparison with the size of the heavens'

Nicolaus Copernicus

A study assessing the performance of optical flow techniques using in-vivo endoscopy images.

For the heading direction determination as described in the previous chapter, a reliable optical flow field is needed. Optical flow algorithms are highly application-specific. This research aims to evaluate different OF algorithms using real endoscopic data. 


\subsection{Introduction}

Optical flow (OF) nowadays is a commonly used technique for vision-based navigation $[72,73]$. It basically entails computation of a displacement field by either solving the optical flow equation (OFE) or by matching distinctive features between two images. Navigation subtasks that have proven to be feasible using OF are, among others, visual odometry, distance estimation, obstacle avoidance and object tracking $[74,75,76]$. Optical flow basically entails the computation of feature or pixel displacements between two 2D images, in order to determine the 3D relative motion between objects [72]. This $3 \mathrm{D}$ relative motion may also be the camera motion with respect to the scene.

A navigation algorithm for a robotized flexible endoscopy system is being developed $[46,77]$. Flexible endoscopes are used for inspection and interventions in a variety of applications, from the industrial to the medical field. Our focus lies on the medical application range. The goal of developing a robotized flexible endoscopy system is to increase the efficiency of the endoscopic procedures by facilitating introduction and fixation of flexible endoscopes. During a standard endoscopic procedure, the endoscope is inserted up to the end of the organ of choice first, and diagnosis and therapy are conducted upon retraction. We think that the largest gain in efficiency can be obtained in the insertion phase of the endoscopic procedure, which is why our navigation algorithm will focus on endoscope insertion.

The OF algorithm is only part of the complete navigation algorithm. The contribution of OF in a navigation algorithm for flexible endoscopes will be estimation of camera motion [45]. However, one of the most mentioned disadvantages of OF-based navigation algorithms is the lack of robustness in (real-time) navigation performance [78]. Solutions to improve robustness in OF-based navigation include, but are not limited to: preprocessing of the images, outlier detection of the OF field, and improving feature detection by parameter optimization [73]. Several systematic comparisons between OF algorithms have been made earlier, such as [79, 80]. Baker et al. proposed a new systematic evaluation methodology for OF algorithms [81]. However, as McCane et al. stress, in performance evaluations of OF algorithms it is vital that real scenes are evaluated for a valid conclusion [82]. McCarthy et al. emphasize that vision-based algorithms need to be tested and compared in a manner that considers the context of the system [80]. OF-based navigation algorithms are highly application-specific, and need to be evaluated with regard to their ultimate application.

The contribution of the current work is twofold. First, a generic framework for optical flow algorithm comparison is proposed here. The need for such a framework stems on the one hand from the application-specificity of OF algorithms, and on the other hand from the lack of generality in current frameworks. The second contribution of this work is the testing of this framework for the application in flexible endoscope navigation. 


\subsection{Related work in endoscopy}

We conducted a literature review earlier regarding automated visual navigation algorithms for flexible endoscopes [83]. In the current paper, we will specifically discuss additional related work in which OF is used for endoscopic applications.

Mori et al. have employed optical flow on human bronchoscopy (endoscopy in the lungs) images for viewpoint and view direction estimation of a bronchoscope [59, 54]. Goal was to register this information on pre-procedural X-ray computed tomography (CT) images by solving for the epipolar constraint. If the viewpoint and view direction are known in real-time, an assistive system for diagnosis can be developed. While their results are promising, the optical flow computation method and whether it was compared to others was not disclosed.

Bell et al. did use different OF algorithms to learn the camera pose from the optical flow between images made by an endoscope $[84,85]$. Artificial Neural Networks were employed in a learning algorithm and investigated under differing illumination circumstances and using two separate image partitioning techniques. Similar to ours, their goal was to use the visual information as feedback in a closed-loop control algorithm for a robotic endoscope. Their findings support the use of OF-based camera pose estimation. However, the experimental setup was rather limited, and not representative for the real applications. Experiments were done on a plastic model of the colon. Plastic model images differ significantly from real images, especially in terms of texture, as can be seen in 4.1. It is therefore unknown what this technique will do in a more realistic environment.
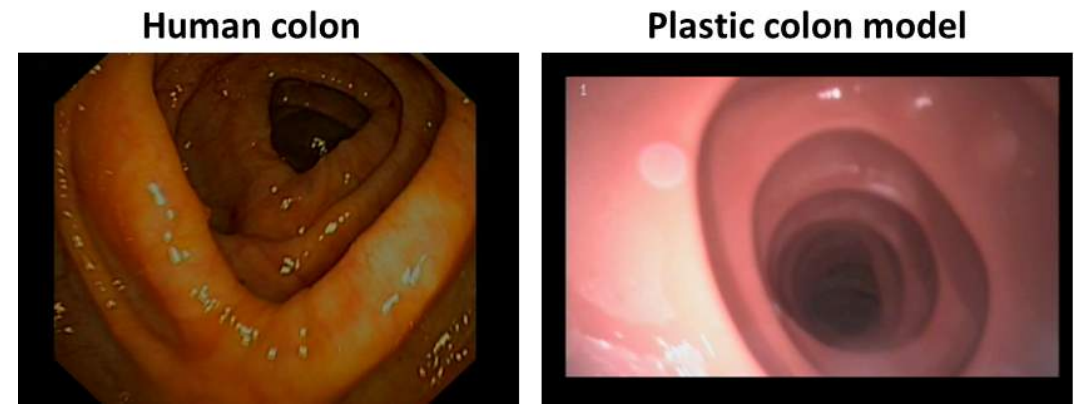

Figure 4.1: Images from a human colon (left) and a plastic colon model (right). Note the differences in texture, structure of the colon and illumination.

Optical flow is used on real images from the human body in other imaging systems, specifically in capsule endoscopy [86]. Aim of this research was to establish endoscope rotation from endoscopic images for 6 degrees of freedom (DOF) pose estimation, which works well according to the results. However, the choice of the OF algorithm was not based on a systematic analysis, but seemed to be prompted by several assumptions of which some are debatable. For instance, the use of SIFT was 


\begin{tabular}{l||ll}
$\begin{array}{l}\text { Image } \\
\text { pair }\end{array}$ & Properties & Category \\
\hline $\mathbf{1}$ & & \\
$\mathbf{2}$ & No artifacts, clean bowel & Easy \\
$\mathbf{3}$ & Nearby wall with high illumination & Hard \\
$\mathbf{4}$ & Fluid, motion blur & Medium-hard \\
$\mathbf{5}$ & Colitis, part blur & Medium \\
\hline
\end{tabular}

Table 4.1: Properties of image pairs.

ruled out because 'endoscopic images are smooth', which would complicate feature detection. Yet, SIFT is known for its blob-like feature tracking qualities (see Section 4.3), and in endoscopic images blobs may occur frequently. The smoothness of the scene therefore does not rule out the applicability of SIFT beforehand, especially since preprocessing might enhance the detailedness of the images when desired.

Further applications of OF in real images in the human body mostly include image mosaicking for better diagnostics and therapeutics [87]. Since these applications differ much from ours, we will not elaborate on this further.

The remainder of this paper will be structured as follows. In Section 4.3 our methods will be explained. The data selection is first described, followed by an elaboration on the preprocessing methods, the different algorithms and the postprocessing method. The framework for comparison and the other materials that were used are discussed next. In the Results section (Section 4.4), we will present our findings per optical flow algorithm. Finally, we will end with the Discussion and Conclusions.

\subsection{Approach}

\subsubsection{Data selection}

Different optical flow algorithms were used to find point correspondences between image pairs. Representative image pairs were obtained from real colonoscopy exams. Each image pair consisted of two images that were shifted with respect to each other. Colonoscopic images have a diversity of appearances depending on the state of the tissue (e.g., inflamed or not), and on the conditions during imaging (e.g. motion blur, possible wall view). Image pairs were selected in such a way, that these differing situations were well-covered. This formed the benchmark data set to test the OF algorithms. The image pairs were qualitatively categorized for later reference. The optimal parameter setting for each OF algorithm was established first, and next, the best algorithm was chosen.

In the Introduction (Section 4.1) we briefly stated that automated navigation will be implemented in endoscopic procedures. Of all the endoscopic procedures, the most difficult images to use for automation are produced during colonoscopies. Therefore, we are using human colonoscopy images to validate our results. The chosen test im- 
ages are displayed in Figure 4.2, details are listed in Table 4.1.

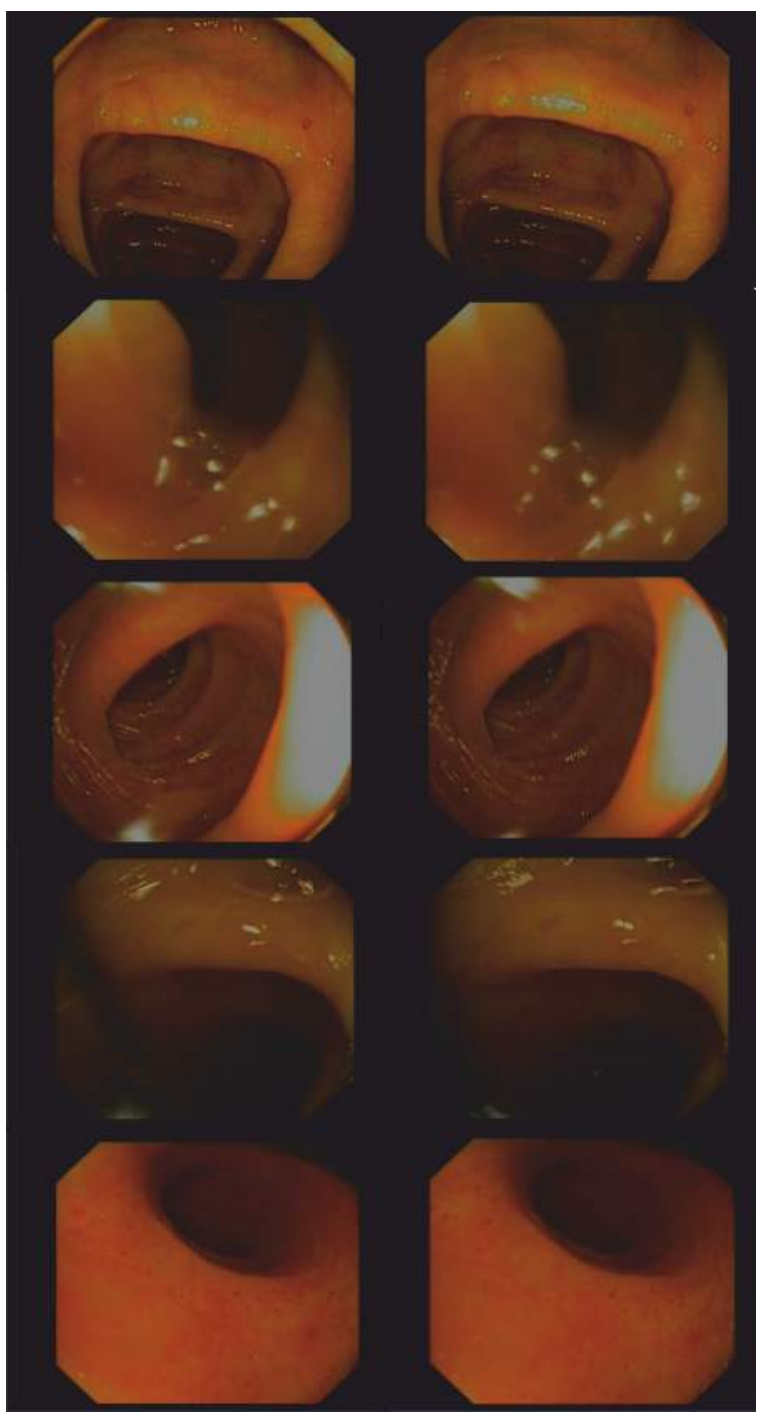

Figure 4.2: The used image pairs in the test data set.

\subsubsection{Preprocessing methods}

Optimal (pre-)processing and parameter settings need to be determined before an objective comparison can take place [88]. It has been shown that different filters influence navigation results from optical flow analysis greatly (e.g. [80]). In our implementation, 
preprocessing options can be changed for each algorithm. A Principal Component Analysis (PCA, [65]) can optionally be performed, comparable to [89]. This option is explored since some color channels might contain more textural information than others. A PCA helps to identify the most relevant information. In addition, histogram equalization is investigated for viability as a preprocessing option since differing illumination circumstances exist in the colon. In 4.3, an overview of the effect of preprocessing is provided.

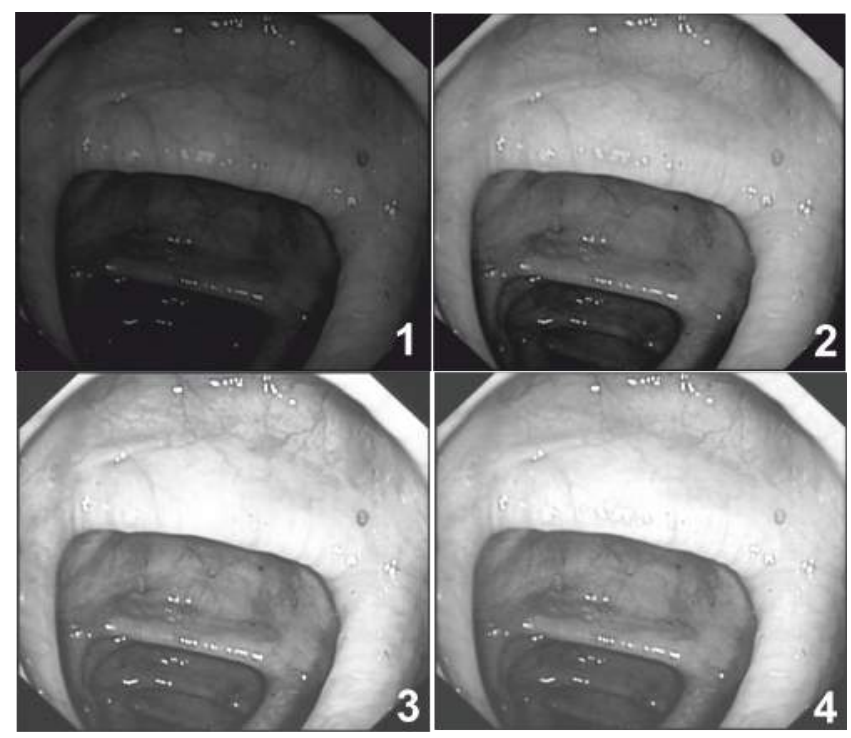

Figure 4.3: The effect of different preprocessing options on an example image. The top row is without histogram equalization, the bottom with. Option 2 and 4 are the ones where also a PCA was applied.

\subsubsection{Optical flow methods}

\section{Lucas-Kanade optical flow}

We selected three different OF algorithms that represent three classes of commonly used OF approaches. One approach is to minimize the Optical Flow Equation (OFE) using the constant brightness assumption. Let the image intensity be given by $I(x, y, t)$ for pixel positions $(x, y)$ over time $t$, and let a camera movement with respect to the environment after time $d t$ results in a pixel displacement $(d x, d y)$. If the objects in the environment are assumed constant, it can be stated that:

$$
\frac{\partial I}{\partial x} d x+\frac{\partial I}{\partial y} d y+\frac{\partial I}{\partial t} d t=0
$$


If we now define the pixel displacements by dividing by $d t$, so $u=\frac{d x}{d t}$ and $v=\frac{d y}{d t}$, the OFE becomes

$$
-\frac{\partial I}{\partial t}=\frac{\partial I}{\partial x} u+\frac{\partial I}{\partial y} v
$$

The OFE minimization method is best represented by the Lucas-Kanade algorithm [61], since it is fast, relatively simple and the most well-known. This algorithm has the aim to minimize the sum of squared error between the template and the image, resulting in a set of warps. Lucas-Kanade is known to suffer from robustness issues when large movements are present between the images. This problem is - partly - solved when using the pyramidal implementation of the algorithm [90]. Therefore, another addition is to implement this algorithm in a dense fashion, in which the optical flow is computed for each pixel of an image. This detailed computation should improve robustness. Subsequently, only the 500 most reliable OF results are taken into account to reduce the computational load. Reliability is established through minimizing the sum of squared differences (SSD) and dividing this by the minimum eigenvalue. In summary, the implementation works as follows:

1. Convolve both input images with a directional Gauss-filter with width $\sigma$ for the $x$ and $y$-directions to obtain $\frac{\partial I}{\partial x}$ and $\frac{\partial I}{\partial y}$

2. Subtract the first from the second image to obtain $\frac{\partial I}{\partial t}$

3. Obtain the matrix $A A=A^{\prime} A=\left[p_{1} p_{2} ; p_{2} p_{3}\right]$ by convolving $\frac{\partial I}{\partial x}^{2}, \frac{\partial I}{\partial y}^{2}$ and $\frac{\partial I}{\partial x} \frac{\partial I}{\partial y}$ with a Gauss-filter with width $\sigma$, resulting in the parameters $p_{1}, p_{3}$ and $p_{2}$, respectively.

4. Compute the vector $B=A p_{2}^{\prime}=[B 1, B 2]^{T}$ by convolving $\frac{\partial I}{\partial x} \frac{\partial I}{\partial t}$ and $\frac{\partial I}{\partial y} \frac{\partial I}{\partial t}$ with a Gauss filter, width $\sigma$.

5. Estimate $u$ and $v$ by solving consecutively:

$$
\begin{gathered}
D=\frac{1}{p_{1} p_{3}-p_{2}^{2}} \\
u=\left(p_{3} B 1-p_{2} B 2\right) D \\
v=\left(p_{1} B 2-p_{2} B 1\right) D
\end{gathered}
$$

6. Now compute the confidence levels of each flow vector by minimizing the SSD...

$$
S S D_{\min }=p_{1} u+p_{2} v-p_{3}^{2}
$$

7. ... and dividing by the minimum eigenvalue $\lambda \min$ :

$$
\begin{gathered}
L=\sqrt{p_{1}^{2}-2 p_{1} p_{3}+4 p_{2}^{2}+p_{3}^{2}} \\
\lambda_{\text {min }}=\frac{p_{1}+p_{3}-L}{2}
\end{gathered}
$$

8. The confidence level can now be computed for each pixel, and by sorting and taking the $N$ minimum values, the $N$ most reliable flow vectors are found.

Note that it is customary in almost every other paper on the subject to implement a Lucas-Kanade algorithm using Shi-Tomasi features (e.g. [84, 85, 86]), resulting in a sparse flow field and features less suited for medical image applications. 


\section{Horn-Schunck optical flow}

In addition, we chose a method based on a smoothness constraint, because navigating through the colon may be considered a 'smooth' motion. Solving the OFE using the smoothness constraint is done in the methods of Horn-Schunck [62] and Nagel [91]. The advantage of the method of Horn and Schunck over Nagel's method is that the used smoothness constraint is not depending on finite differences and thus more widely applicable. For the minimization of Horn-Schunck the following equation is used:

$$
\begin{aligned}
& E=\iint\left(I_{x} u+I_{y} v+I_{t}\right)^{2} d x d y+ \\
& \quad \alpha \iint\left\{\left(\frac{\partial u}{\partial x}\right)^{2}+\left(\frac{\partial u}{\partial y}\right)^{2}+\left(\frac{\partial v}{\partial x}\right)^{2}+\left(\frac{\partial v}{\partial y}\right)^{2}\right\} d x d y
\end{aligned}
$$

The Horn-Schunck algorithm is implemented in the original (dense) form as described in [62]. Because of the iterative nature of the algorithm, computation time can be impressive. To limit the number of flow vectors, and with that computation time, matches were chosen from a grid (every 5th pixel), resulting in a number of 744 flow vectors.

\section{SIFT feature-based approach}

A third approach is to use a point feature detection algorithm. If features are detected in two consecutive images, a displacement vector between them can be defined. Multiple displacement vectors then form a sparse optical flow field. SIFT, Scale-Invariant Feature Transform [63, 92], was chosen for its blob-like feature tracking qualities. SIFT is known to provide excellent results, but at high computational cost (e.g. [93, 94]). SURF [69] and ORB [95] are examples of less costly, SIFT-derived methods and may be used as alternatives. Since their results are slightly less accurate, these will only be considered if SIFT turns out to be the most effective method from the current research. The real-time requirement will be taken into account in a later stage of our research.

In the SIFT algorithm, features are detected and matched based on their mathematical properties stored in the descriptor [63]. The inherent property of SIFT features, that it matches blob-like features at different scales accurately, also leads to a computationally intensive algorithm. However, it is the feature matching step that takes up most of the computation time. Therefore we investigate an alternative algorithm that first finds SIFT key points in the first image, and then tracks these points using the Lucas-Kanade method in the second image. In our algorithm, one feature detector and one tracking method are chosen, and all of them can be combined. Table 4.2 provides an overview of the selected combinations and the parameter setting ranges that were tested during our experiments.

For the LK and HS options, a Gauss filter is employed (see section 4.3.3). The width of the peak of this filter is described by the parameter sigmaG. The pyramid level parameter simply represents the number of levels in the pyramid, as described by Bouguet et al. [90]. In the SIFT algorithm, a match between two features is among others determined by the so-called 'distratio'. This ratio is used to test the distance of the nearest neighbor to the second nearest neighbor. If this distance is larger than the 


\begin{tabular}{|c|c|c|c|c|}
\hline $\begin{array}{l}\text { Feature } \\
\text { selector }\end{array}$ & $\begin{array}{l}\text { Flow } \\
\text { method }\end{array}$ & Acronym & Parameters & Range of settings \\
\hline SIFT & $\begin{array}{l}\text { Lucas- } \\
\text { Kanade }\end{array}$ & SIFT-LK & $\begin{array}{l}\text { sigmaG, } \\
\text { pyramid } \\
\text { level }\end{array}$ & $\begin{array}{l}\text { sigmaG }=0.5: 1: 3.5 \\
\text { pyramid level }=2: 5\end{array}$ \\
\hline & Descriptor & SIFT & distRatio & distRatio = 0.3:0.1:1.0 \\
\hline \multirow[t]{2}{*}{$\begin{array}{l}\text { None } \\
\text { (dense) }\end{array}$} & $\begin{array}{l}\text { Horn- } \\
\text { Schunck }\end{array}$ & $\mathrm{H}-\mathrm{S}$ & $\begin{array}{l}\text { sigmaG, al- } \\
\text { pha, Ethres }\end{array}$ & $\begin{array}{l}\text { sigmaG }=0.5: 1: 3.5 \\
\text { alpha }=\left[\begin{array}{lll}0.1 & 1 & 10\end{array}\right] \\
\text { Ethres }=\left[\begin{array}{ll}1 e-1 & 1 e-3 \\
1 e-6\end{array}\right]\end{array}$ \\
\hline & $\begin{array}{l}\text { Lucas- } \\
\text { Kanade }\end{array}$ & LK & $\begin{array}{l}\text { sigmaG, } \\
\text { pyramid } \\
\text { level }\end{array}$ & $\begin{array}{l}\text { sigmaG }=0.5: 1: 3.5 \\
\text { level = 2:5 }\end{array}$ \\
\hline
\end{tabular}

Table 4.2: Possible combinations of feature detectors and flow methods, with their respective tested parameters.

distratio times the distance to the nearest neighbor, then the match is rejected. Alpha in the Horn-Schunck algorithm regulates the smoothness of the flow (larger values of alpha lead to a smoother flow). The Ethres is the minimum velocity difference.

\subsubsection{Framework for comparison of OF methods}

\section{Performance criterion}

In order to optimize the performance of the different algorithms a performance criterion is needed. Since the goal is navigation, the criterion should express the capability of the OF field to find the second pose of the camera relative to the first pose, i.e. the translation and the rotation. A possibility could be a criterion that expresses the ability of the OF field to find the focus of expansion [45]. The focus of expansion is a direct measure of the heading direction of the camera motion, and as such relevant. The downside of a measure that is derived from the focus of expansion is that it is only applicable to translational movements, and not to rotational movements.

Therefore, a better performance criterion would be one which is derived from the fundamental matrix that is associated with the image pairs. The fundamental matrix is a $3 \times 3$ matrix that in its turn is derived from the essential matrix. The essential matrix lays down the so-called epipolar geometry of the pose of one camera relative to the other, and as such encodes both the rotation and the translation of this relative pose (see 5 or $[73,96]$ for a more elaborate explanation). So, preferably, our performance measure would directly be related to the essential matrix. Unfortunately, the essential matrix cannot be obtained from the OF field without knowledge of the intrinsic parameters of the camera. The fundamental matrix expresses the epipolar geometry only in terms of pixel coordinates of one image in relation to pixel coordinates of the other image. It is therefore indirectly related to the relative rotation and translation of the two 
cameras, and it has the advantage that it can be estimated from the OF field directly via, for instance, the so-called eight point algorithm.

In the next section, we elaborate on the computation of the fundamental matrix and how comparison of these matrices can be used for optical flow algorithm comparison.

Fundamental matrix comparison Consider a 3D point $\mathbf{X}$, projected as $\mathbf{x}$ in image $I_{1}$, and in image $I_{2}$ as point $\mathbf{x}^{\prime}$. These two image points are represented in homogeneous coordinates, such that $\mathbf{x}=\left[u_{i}, v_{i}, 1\right]^{T}$ and $\mathbf{x}^{\prime}=\left[u_{i}^{\prime}, v_{i}^{\prime}, 1\right]^{T}$. The epipolar constraint is expressed using the fundamental matrix $\mathbf{F}$ as follows:

$$
\vec{x}^{\prime} \mathrm{F} \vec{x}=0
$$

F can be considered a correlation between the two images that can be computed from at least 8 corresponding image points [73]. When $\mathbf{F}$ is described as:

$$
\mathbf{F}=\left[\begin{array}{lll}
f_{1,1} & f_{1,2} & f_{1,3} \\
f_{2,1} & f_{2,2} & f_{2,3} \\
f_{3,1} & f_{3,2} & f_{3,3}
\end{array}\right]
$$

eq. 4.4 can be rewritten to:

$$
\left[\begin{array}{lllllllll}
u_{i} u_{i}^{\prime} & v_{i} u_{i}^{\prime} & u_{i}^{\prime} & u_{i} v_{i}^{\prime} & v_{i} v_{i}^{\prime} & v_{i}^{\prime} & u_{i} & v_{i} & 1
\end{array}\right] \mathbf{f}=0
$$

Here, we define:

$$
\mathbf{f}=\left[\begin{array}{lllllllll}
f_{1,1} & f_{1,2} & f_{1,3} & f_{2,1} & f_{2,2} & f_{2,3} & f_{3,1} & f_{3,2} & f_{3,3}
\end{array}\right] .
$$

Assuming we know $n$ pixel correspondences (or measurements), the matrix form of eq. 4.7 is given by:

$$
\left[\begin{array}{ccccccccc}
u_{1} u_{1}^{\prime} & v_{1} u_{1}^{\prime} & u_{1}^{\prime} & u_{1} v_{1}^{\prime} & v_{1} v_{1}^{\prime} & v_{1}^{\prime} & u_{1} & v_{1} & 1 \\
\vdots & \vdots & \vdots & \vdots & \vdots & \vdots & \vdots & \vdots & \vdots \\
u_{n} u_{n}^{\prime} & v_{n} u_{n}^{\prime} & u_{n}^{\prime} & u_{n} v_{n}^{\prime} & v_{n} v_{n}^{\prime} & v_{n}^{\prime} & u_{n} & v_{n} & 1
\end{array}\right] \mathbf{f}=\mathbf{U f}=0
$$

F can then be computed by solving eq. 4.8 using at least 8 point pairs. Further theoretical background and proofs are given in [73].

Ground truth The validation of this framework can be done by comparing OF results to the ground truth, which is the unknown 3D scene structure and the relative camera motion - unknown as well. In this research, we therefore regard manually matched point features $\mathbf{x}_{U}$ as the ground truth. For each image pair, on average 38 matching pixel locations were manually indicated (range 17-50, needed was a minimum number of 8, red.). The correspondence vector between the two pixel locations displayed the displacement of a pixel, and all correspondence vectors together resulted in a (sparse) optical flow field. An example result of the matches between images is displayed in Figure 4.4. 


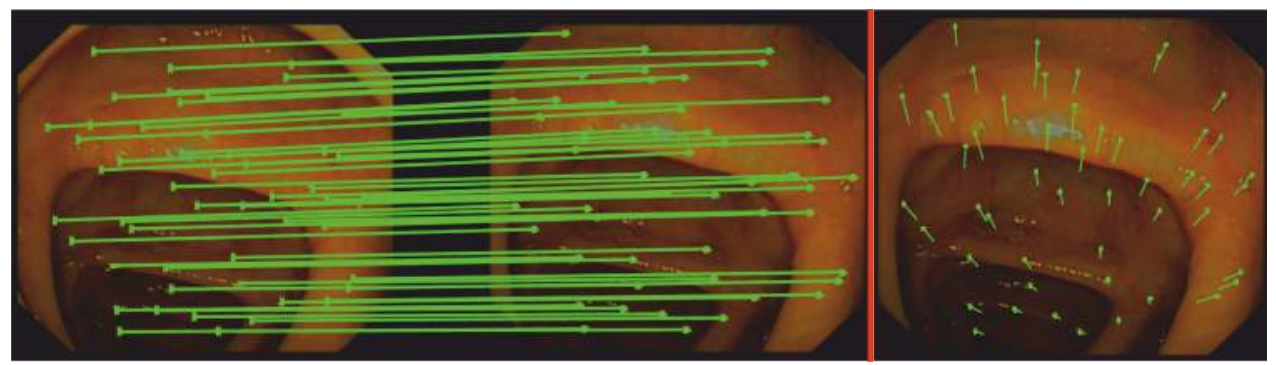

Figure 4.4: Manually matched point features in two shifted images (left) and represented as a vector field in the second image (right).

Performance comparison An objective comparison of performance can only be realized if the resulting fundamental matrix from the ground truth can be objectively compared to the fundamental matrix resulting from the automatically matched point features (or optical flow results).

Basically, when comparing all different OF techniques with the different parameter options to the chosen ground truth, we are looking for a minimum difference between the obtained fundamental matrix $\mathbf{F}_{2}$ and the ground truth fundamental matrix $\mathbf{F}_{1}$. This minimum difference can be found by the measure of Laveau that Zhang summarized in [97]. The computation works as follows:

1. Choose a random point $\mathbf{m}$ from the corresponding point pairs.

2. Compute the epipolar line of $\mathbf{m}$ in the second image using $\mathbf{F}_{1}$ through $\mathbf{F}_{1} \mathbf{m}$.

3. Check if the epipolar line intersects the second image. If not, return to $\mathbf{1}$.

4. Choose a random point $\mathbf{m}^{\prime}$ on the epipolar line. $\mathbf{m}$ and $\mathbf{m}^{\prime}$ correspond with respect to $\mathbf{F}_{1}$ (Figure 4.5).

5. Compute the epipolar line of $\mathbf{m}$ in the second image $\left(\mathbf{F}_{2} \mathbf{m}\right)$ and then determine the distance $\mathbf{d}_{1}^{\prime}$ between this line and point $\mathbf{m}^{\prime}$.

6. Compute the epipolar line of $\mathbf{m}^{\prime}$ in the first image, i.e. $\mathbf{F}_{2}^{\mathrm{T}} \mathbf{m}^{\prime}$. Determine the distance between this line and point $\mathbf{m}$ (distance $\mathbf{d}_{1}$ ).

7. Switch $\mathbf{F}_{\mathbf{1}}$ and $\mathbf{F}_{\mathbf{2}}$ and go through $\mathbf{2}$. to $\mathbf{6}$. again. These steps result in $\mathbf{d}_{\mathbf{2}}$ and $\mathbf{d}_{\mathbf{2}}^{\prime}$.

8. Iterate the steps above until $N$ iterations are completed.

9. The average distances of all $d$ 's expresses the difference between the two fundamental matrices.

The parameter settings resulting in the minimal distances between $\mathbf{F}_{\mathbf{1}}$ and $\mathbf{F}_{\mathbf{2}}$ were chosen for each algorithm. The algorithm resulting in the minimal distance was considered the best one.

Postprocessing The fundamental matrix is computed from all flow vectors, but outlying vectors influence the result heavily. The OF uncertainty measure does make sure that a certain weight is assigned to certain OF vectors, but this does not remove completely unreliable flow vectors. Therefore, post-processing is introduced with the aim 

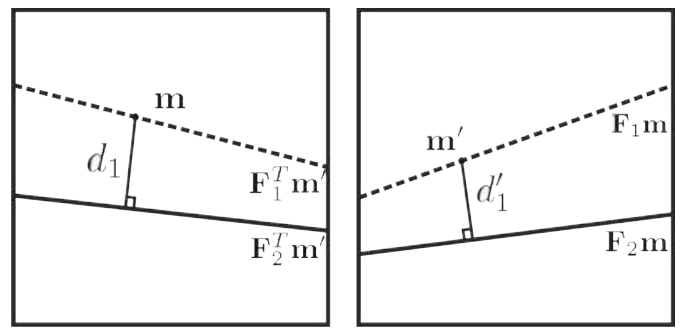

Figure 4.5: Schematic representation of how the distances between $\mathbf{F}_{1}$ and $\mathbf{F}_{2}$ can be interpreted.

to remove the outlying vectors, although it will increase computational time. For outlier detection in the sparse method, the RANSAC algorithm was used [66].

RANSAC implementation Outliers were detected in an iterative fashion by first initializing the fundamental matrix $\mathbf{F}$. Then, eight point pairs were randomly taken from the data set and the fundamental matrix $\mathbf{f}$ was computed from them by using the normalized 8-point algorithm as described in Section 4.3.4. The consensus of $\mathbf{f}$ was computed (the fraction of pairs falling into the category 'inlier' using this specific $\mathbf{f}$ ). If the fitness of $\mathbf{f}$ exceeded that of $\mathbf{F}$, $\mathbf{f}$ was accepted as the new $\mathbf{F}$. This was done at most 2000 times per $\mathbf{F}$ computation (less if a solution was found earlier).

\subsubsection{Algorithm selection - evaluation criteria}

All image pairs were manually annotated and automatically analyzed using the described pre-processing options, OF algorithms and post-processing options. The results from these automated analysis were evaluated to choose the best combination of methods and settings based on the following criteria:

- Visual inspection of the OF results.

- All results from the Horn-Schunck flow method were inferior to those of the other methods. Therefore, this algorithm was not analyzed further (see section 4.5).

- The resemblance of $\mathbf{F}_{1}$ to $\mathbf{F}_{2}$, which is measured by the distance between them.

- The ratio between the number of inlying matches and the total number of key points

- The percentage of frames for which a successful fundamental matrix estimation could be done (i.e. enough inliers present in images)

- Computational effort with the real-time application in mind; the number of seconds per computation

\subsubsection{Materials}

Images were acquired using an Olympus CF180 colonoscope with an Olympus CV180 Evis Exera II video processor. This type of colonoscope produces $576 \times 768$ pixel im- 
ages with a chosen frame rate of 25 frames per second. It has a field of view of $170^{\circ}$ and a field depth of 3 to $100 \mathrm{~mm}$. All algorithms were implemented using Matlab R2014b (The Mathworks Inc., Natick, MA, USA).

\subsection{Results}

\subsubsection{Lucas-Kanade parameter optimization}

\begin{tabular}{|c|c|c|c|c|}
\hline $\begin{array}{l}\text { Image } \\
\text { Pair }\end{array}$ & $\begin{array}{l}\text { Succes } \\
\text { (\%) }\end{array}$ & $\begin{array}{l}\text { Median } \\
\text { inliers } \\
\text { (IQR) }\end{array}$ & $\begin{array}{l}\text { Minimum } \\
\text { distance } \\
\text { (mean) }\end{array}$ & $\begin{array}{l}\text { Correspon- } \\
\text { ding parame- } \\
\text { ters }\end{array}$ \\
\hline 1 & 83.3 & $9(1)$ & $\begin{array}{l}36.75 \\
(164.01)\end{array}$ & $\begin{array}{l}\text { sigmaG }=1.5 \\
\text { level = } 5\end{array}$ \\
\hline 2 & 72.9 & $9(3)$ & $\begin{array}{l}63.19 \\
(157.63)\end{array}$ & $\begin{array}{l}\text { sigmaG }=1.5 \\
\text { level =3 }\end{array}$ \\
\hline 3 & 82.3 & $12(5.8)$ & $\begin{array}{l}40.92 \\
(141.91)\end{array}$ & $\begin{array}{l}\text { sigmaG }=2.5 \\
\text { level }=2\end{array}$ \\
\hline 4 & 81.3 & $9(2)$ & $\begin{array}{l}59.61 \\
(150.86)\end{array}$ & $\begin{array}{l}\text { sigmaG }=0.5 \\
\text { level }=4\end{array}$ \\
\hline 5 & 88.5 & $9(2)$ & $\begin{array}{l}35.97 \\
(149.93)\end{array}$ & $\begin{array}{l}\text { sigmaG }=0.5 \\
\text { level }=2\end{array}$ \\
\hline
\end{tabular}

Table 4.3: LK optical flow outcome for all image sequences. IQR = InterQuartile Range. Note that the number of matches in this OF method always equals 500 , since we select the 500 most reliable features.

Optimal parameter settings needed to be found for the width of the Gauss peak (sig$\mathrm{maG}$ ) and the number of levels of the pyramid. In terms of outcome, the best sigmaG is 1.3 on average (Table 4.3), although the value for sigmaG does not influence the outcome heavily (Figure 4.6, left). The best level setting appears to be around 3. This is not the maximum possible number of levels, which helps in computational time. It was shown that the higher the level, the higher the computational load (Figure 4.6, right).

The best preprocessing option was 3 or 4 (Figure 4.7). This means histogram equalization has added value, but the added value of a one-channel PCA is probably limited. Since the additional computation of a PCA does take time and the plot looks a little more promising, preprocessing option 3 is chosen (histogram equalization without PCA).

\subsubsection{SIFT parameter optimization}

The distance ratio within which a feature pair would be considered a match (distRatio) needed to be set. A distRatio value of 0.8 would be resulting in the smallest distance 


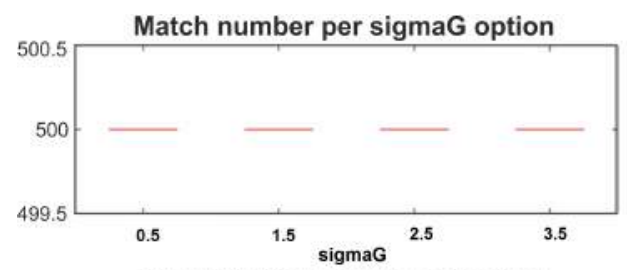

Inlier number per sigmaG option

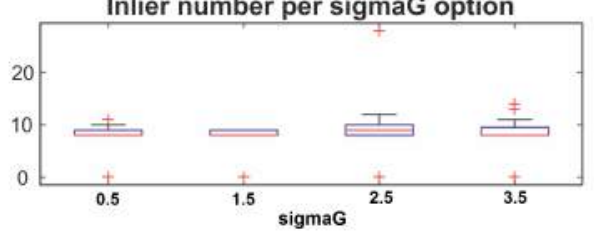

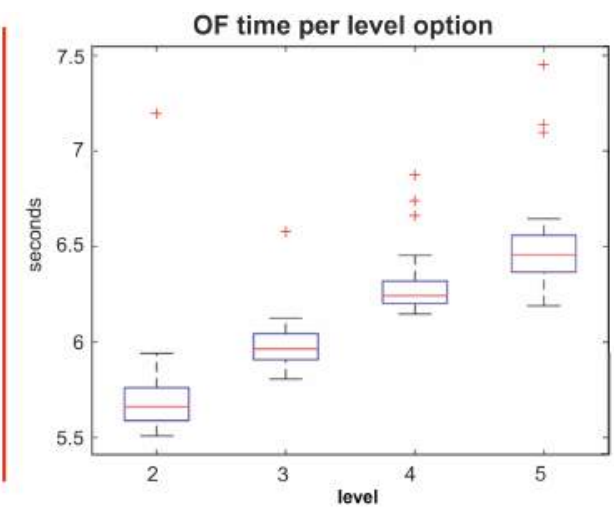

Figure 4.6: Left: the number of matches and inliers per sigma option. Note that the number of matches is fixed at 500 again. Right: optical flow computation times sorted by level (seconds).

NB: algorithm implementation was not optimized for speed. Example shown are resulting times from image pair 1 .
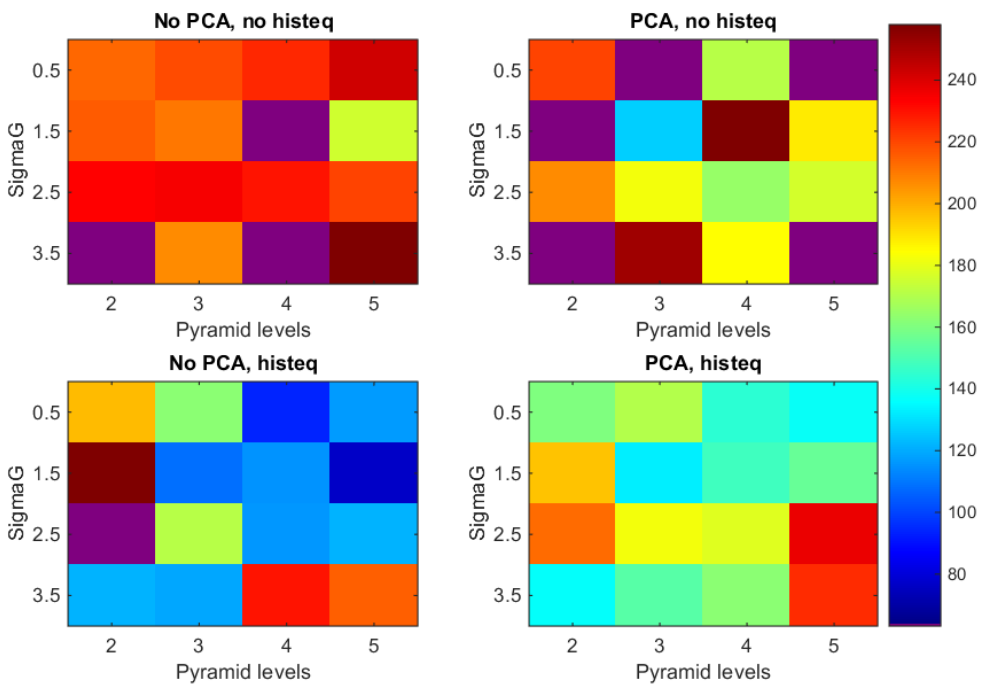

Figure 4.7: Color plots showing the distances between fundamental matrices per preprocessing setting for the LK algorithm. The parameters vary along the axes. The blue values are the minimal (and desirable) values. Example shown are resulting distances from image pair 2, for which preprocessing options 3 and 4 are the most blue. Note that purple represents a value of 0 , which means no outcome was possible. 


\begin{tabular}{|c|c|c|c|c|c|}
\hline $\begin{array}{l}\text { Image } \\
\text { Pair }\end{array}$ & $\begin{array}{l}\text { Succes } \\
(\%)\end{array}$ & $\begin{array}{l}\text { Median } \\
\text { matches } \\
\text { (IQR) }\end{array}$ & $\begin{array}{l}\text { Median } \\
\text { inliers } \\
\text { (IQR) }\end{array}$ & $\begin{array}{l}\text { Minimum } \\
\text { distance } \\
\text { (mean) }\end{array}$ & $\begin{array}{l}\text { Correspon- } \\
\text { ding parame- } \\
\text { ters }\end{array}$ \\
\hline 1 & 60.4 & $36(28)$ & $11(2)$ & $\begin{array}{l}40.14 \\
(172.16)\end{array}$ & $\begin{array}{l}\text { sigmaG }=3.5 \\
\text { distRatio }=0.9\end{array}$ \\
\hline 2 & 14.6 & $20(6)$ & $9(1)$ & $\begin{array}{l}88.52 \\
(142.28)\end{array}$ & $\begin{array}{l}\text { sigmaG }=3.5 \\
\text { distRatio }=0.7\end{array}$ \\
\hline 3 & 62.5 & $50(86)$ & $11(4)$ & $\begin{array}{l}28.16 \\
(134.29)\end{array}$ & $\begin{array}{l}\text { sigmaG }=2.0 \\
\text { distRatio }=0.8\end{array}$ \\
\hline 4 & 19.8 & $22(9)$ & $12(5)$ & $\begin{array}{l}43.4422 \\
(106.45)\end{array}$ & $\begin{array}{l}\text { sigmaG }=1.5 \\
\text { distRatio }=0.8\end{array}$ \\
\hline 5 & 33.3 & $44(44.5)$ & $10(1)$ & $\begin{array}{l}88.84 \\
(148.59)\end{array}$ & $\begin{array}{l}\text { sigmaG }=2.5 \\
\text { distRatio }=0.9\end{array}$ \\
\hline
\end{tabular}

Table 4.4: SIFT optical flow outcome for all image pairs. IQR = InterQuartile Range.

4.9 becomes clear that the higher this ratio, the better the outcome. Since some selection in features still is desirable to limit the number outliers, the optimal setting for the distance ratio is chosen to be 0.9 . Figure 4.9, depicting results from a representative image pair, shows that the number of matches is varying greatly between the different settings in distRatio. Nonetheless, the number of inlying matches is more or less constant, as are the distances between the fundamental matrices (Figure 4.10, right).

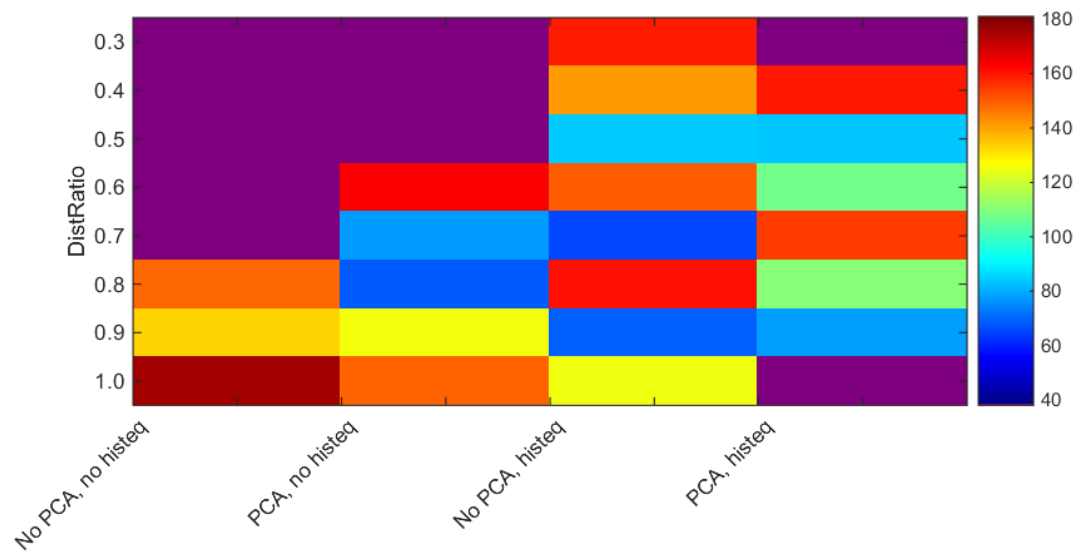

Figure 4.8: Distances displayed in color scale for preprocessing options and distRatio settings in the SIFT algorithm. The blue values are minimal, and therefore better. Note that the purple areas are a distance of 0 , which means no outcome was possible. Example shown from image pair 3. 

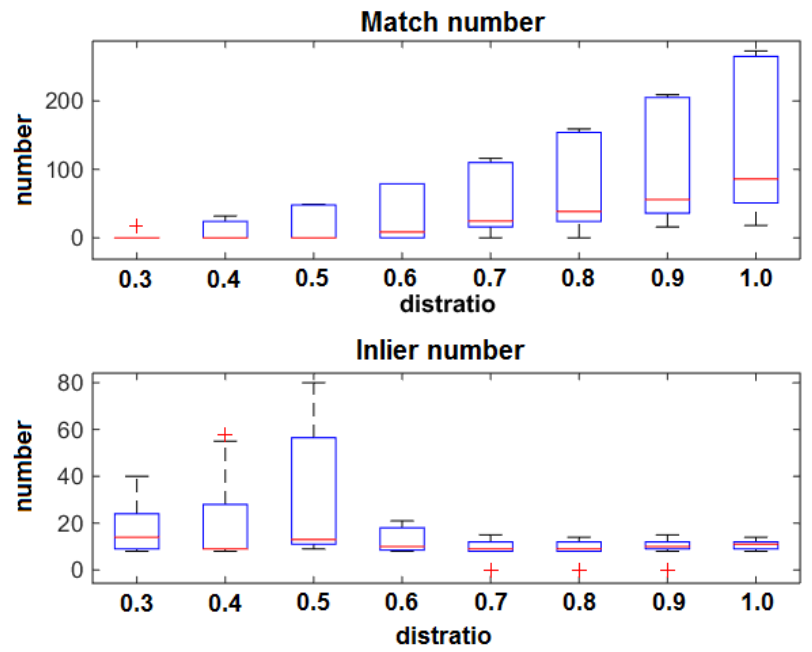

Figure 4.9: Boxplot showing the spread of the matches and inlier numbers per distRatio option for the SIFT algorithm. It shows that the higher the distRatio, the more matches are present, but the number of inliers remains relatively constant. Example shown from image pair 3.

The best preprocessing options were option 3 and 4, (Figure 4.8), since these resulted in the minimal outcome. This means histogram equalization has a positive effect on the SIFT algorithm outcome, but adding a one-channel PCA does not make an enormous difference. Since a PCA means an additional computation step, preprocessing option 3 was chosen (only histogram equalization). Mean computational time was relatively constant(Figure 4.10 , left).

\subsubsection{SIFT - Lucas-Kanade parameter optimization}

Similar to the Lucas-Kanade optimization, sigmaG and level options needed to be set here. From the results (Table 4.5), it appears that sigmaG $=2.7$ is a good value, and the best number of levels is again 3 . The value for sigmaG again does not change the outcome significantly.

The preprocessing does affect outcome greatly. Especially the ratio between number of matches and inliers depends on preprocessing (Figure 4.11). This is however not reflected in the distance color plot of Figure 4.12, in which option 1 scores visibly worse than the others, while it resulted in the highest number of inliers. The best preprocessing option was determined similar to the previous two OF algorithms. For the SIFT-LK algorithm the best results were obtained with histogram equalization and a one-channel PCA (option 4, Figure 4.12). 

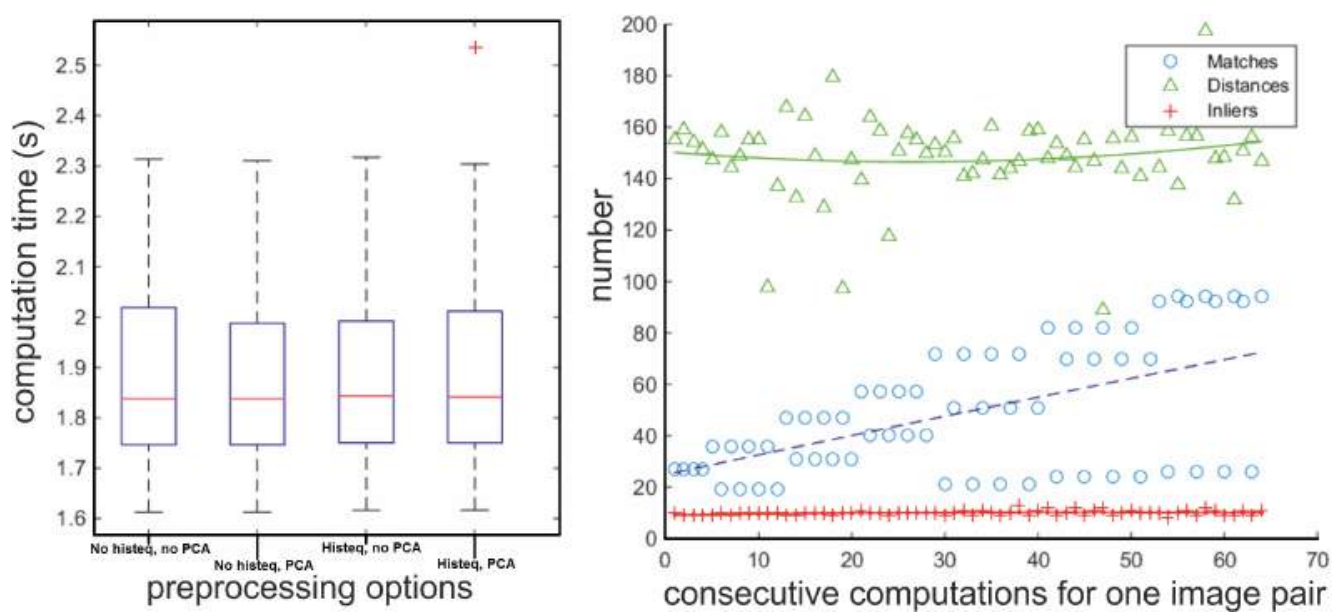

Figure 4.10: Left: an example boxplot of differences in computational time. It is shown that the mean and spread are relatively constant, independent from preprocessing settings. Example shown from image pair 1. Right: SIFT outcome in terms of matches, inliers and distances between fundamental matrices. Example shown from image pair 5 .

\begin{tabular}{|c|c|c|c|c|c|}
\hline $\begin{array}{l}\text { Image } \\
\text { Pair }\end{array}$ & $\begin{array}{l}\text { Succes } \\
(\%)\end{array}$ & $\begin{array}{l}\text { Median } \\
\text { matches } \\
\text { (IQR) }\end{array}$ & $\begin{array}{l}\text { Median } \\
\text { inliers } \\
\text { (IQR) }\end{array}$ & $\begin{array}{l}\text { Minimum } \\
\text { distance } \\
\text { (mean) }\end{array}$ & $\begin{array}{l}\text { Correspon- } \\
\text { ding parame- } \\
\text { ters }\end{array}$ \\
\hline 1 & 100 & $381(159)$ & $12(2.5)$ & $\begin{array}{l}127.53 \\
(183.18)\end{array}$ & $\begin{array}{l}\text { sigmaG }=2.5 \\
\text { level }=2\end{array}$ \\
\hline 2 & 86.5 & $114(26)$ & $10(2)$ & $\begin{array}{l}108.29 \\
(181.51)\end{array}$ & $\begin{array}{l}\text { sigmaG }=3.5 \\
\text { level }=3\end{array}$ \\
\hline 3 & 97.9 & $\begin{array}{l}395 \\
(592)\end{array}$ & $13(4)$ & $\begin{array}{l}32.16 \\
(150.18)\end{array}$ & $\begin{array}{l}\text { sigmaG }=2.5 \\
\text { level }=5\end{array}$ \\
\hline 4 & 90.6 & 191 (137) & $10(2)$ & $\begin{array}{l}70.12 \\
(175.72)\end{array}$ & $\begin{array}{l}\text { sigmaG }=2.5 \\
\text { level }=3\end{array}$ \\
\hline 5 & 81.3 & 75 (236) & $10(2)$ & $\begin{array}{l}70.12 \\
(175.72)\end{array}$ & $\begin{array}{l}\text { sigmaG }=2.5 \\
\text { level }=2\end{array}$ \\
\hline
\end{tabular}

Table 4.5: SIFT-LK optical flow outcome for all image pairs. IQR = InterQuartile Range.

\subsection{Discussion}

Although optical flow algorithms have existed for a long time, the best method for comparison still gives rise to discussion [79]. Benchmark data sets are a common approach for comparison [81], but as stated in the Introduction, optical flow performance is highly application-specific. Aim of the current work was to establish a generic framework for 


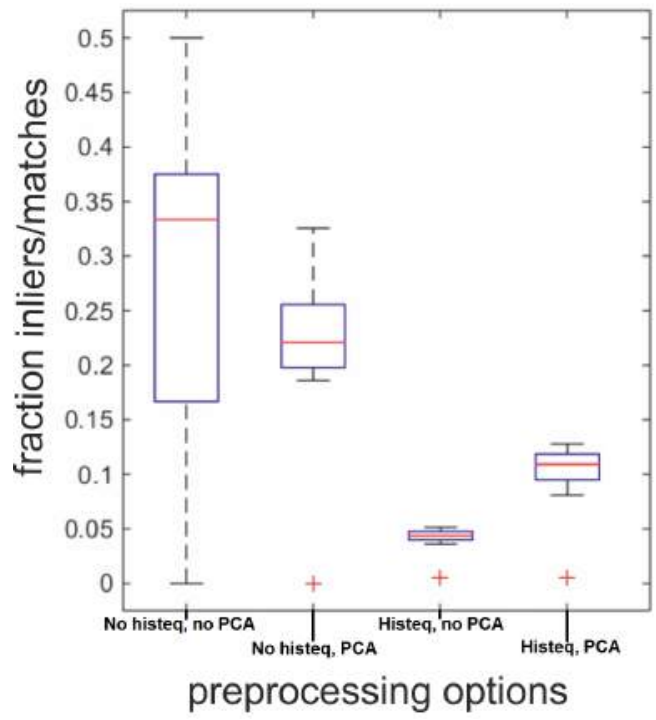

Figure 4.11: Fraction between matches and inliers for SIFT-LK. Example shown from image pair 5.
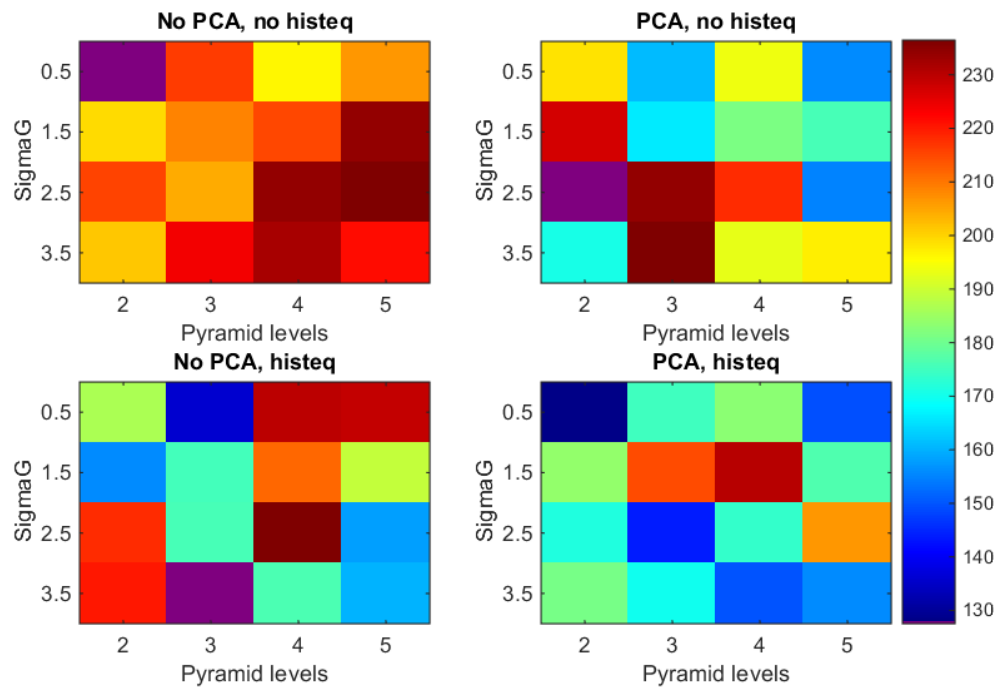

Figure 4.12: Fundamental matrix distances in color plot visualization for the SIFT-LK algorithm. Example shown from image pair 1. 
application-specific optical flow algorithm comparison and to test this framework for the application of flexible endoscope navigation. The proposed framework was a combination of earlier work by others, but has never been used in the context as presented in this paper. Main contribution was the use of fundamental matrix comparison to evaluate optical flow performance.

In general, the framework worked well. The implementation of the proposed framework is relatively straightforward, which is an advantage if others want to use it. Overall, the resulting distances between the fundamental matrices were larger than expected and than the distances found in [97]. This is probably due to the different nature of the images that were used. In [97], real-world images of high quality depicting a static environment were employed. The in-vivo images used in the current research often lack texture, and color and intensity differences can be very similar over multiple images. In addition, artifacts such as fluids, smoke or instruments in the field of view, or motion blur due to fast endoscope movements complicate automated image analysis further.

The parameter optimization and comparison of OF algorithms could be done using the proposed framework. Initially, the Horn-Schunck method was selected reasoning that the motion through human organs was a smooth one. Although this may be true for an average of the total motion, for the situation as observed frame-by-frame this is certainly not the case. Large changes in direction, even within parts of images, exist, which probably caused the Horn-Schunck method to deliver inferior results. In addition, the computational time can become steep fairly quickly, since the method is iterative until it converges to a certain error threshold. Computational time is thus unpredictable, and this method is therefore not deemed suitable for real-time applications.

The smallest fundamental matrix distances were found using the LK algorithm. SIFT had a better match-to-inlier ratio. The succes frame percentage was highest using the SIFT-LK combination. Since the LK algorithm, depending on the implementation, will also be the one with the lowest computational load, this algorithm is considered the best one from the current experiments and for our application. For the algorithms using SIFT features, the best results were obtained the same preprocessing settings which demonstrates consistency in the analysis framework. This furthermore is logical since preprocessing influences feature properties and thus detection performance.

An important tool for algorithm parameter optimization and comparison were the color plots. Two observations can be made regarding these plots. The color purple represents a value of 0 , which means no outcome was possible. When this was the case, it meant that too little inliers were present in the OF field to compute a fundamental matrix. The second observation is that the colors not always form a gradual changing color field. We think the main reasons for this are at the one hand the discrete scale at which parameters were tested, and on the other hand the postprocessing method and the OF robustness. During postprocessing the RANSAC algorithm was applied, which influences the ultimate inlying vectors and therefore, the resulting fundamental matrix. If no postprocessing is used, the reliability of the fundamental matrix estimation is compromised. However, the random element of this method introduces a risk for inconsistent results. We have performed each analysis three times and subsequently 
taken the average to decrease this risk.

The OF algorithms employed in this research are all feature- or pixel-based. A different approach is a region-based method such as block matching [98]. Instead of computing the displacement of a single pixel or feature, the displacement of a region is computed. This region can be established in many ways, a discussion of which falls outside the scope of this paper. However, region-based methods are more sensitive to illumination changes and object occlusions. Unfortunately, both effects are very common in the images used in our application. Another risk that exists for these techniques is a high computational time.

\subsection{Conclusion}

Optical flow algorithms are often compared using a set of benchmark images. Although these comparisons give a good impression of things like computational effort, the quality of the displacement estimation is highly application-specific. An easy-toimplement framework for generic optical flow algorithm comparison was proposed here, which can be employed to establish optical flow performance for every application. In addition, as demonstrated in the current paper, the framework can be employed for parameter optimization within a single optical flow algorithm. Although no regionbased approach was tested here, it is expected that the framework will work in this case, too. However, the fundamental matrix estimation will have to be adapted then.

\subsection{References}

Please refer to the Bibliography at the end of this thesis. 
CHAPTER 5

\section{Navigation in endoscopy: investigation of an optical flow model}

Albert Einstein

Research conducted as a follow-up from the research described in Chapter 3.

This chapter contains the results of an extensive study conducted to investigate the optical flow/focus of expansion applicability in endoscope navigation. It is an evolution of the work described in Chapter 3. Experiments were performed in the lab using different endoscopic environments (i.e. plastic models and animal tissue) and an electromagnetic tracking setup. 


\subsection{Introduction}

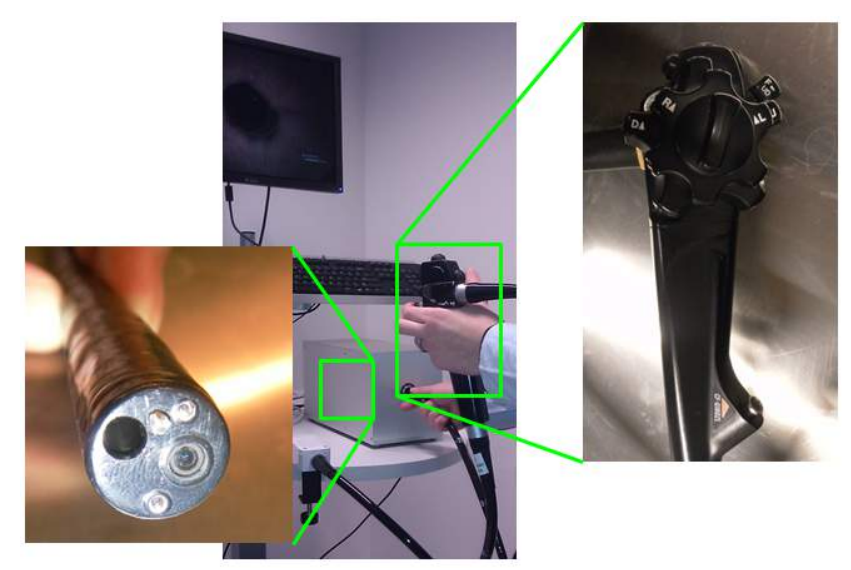

Figure 5.1: Endoscopy where handle and endoscope tip are enlarged to show the steering knobs and camera location.

Flexible endoscopic procedures ('endoscopies') currently are commonly performed medical procedures with the aim of inspecting and/or treating an organ internally using an endoscope (Figure 5.1). Such procedures may provide additional information about the tissue that cannot be seen on magnetic resonance (MR) or computed tomography (CT) images (e.g. tissue color under visible light), but also offer the advantage of being able to directly treat a patient in some cases. Mostly, these procedures are performed on lungs (bronchoscopy), stomach (gastroscopy) or large bowel (colonoscopy) [19]. Typically, an endoscope is inserted until a generally known endpoint is reached. During this insertion, the organ may be inflated if it was collapsed (as is the case in gastroscopy and colonoscopy). When the end-point is reached, the endoscope is retracted slowly, giving the medical doctor the opportunity to inspect the tissue carefully. Especially in colonoscopy often lesions are encountered that could be predecessors of cancerous lesions. In that case, the tissue can directly be taken out for examination (biopsy) or even be removed completely (e.g. polypectomy). The latter completely prevents the cancer from developing and thus is a major contributing factor in the prevention and treatment of colorectal cancer.

If flexible endoscopes could be tele-manipulated or robotized, introducing more degrees of freedom (DOFs) to the user, this could offer the user the opportunity to treat increasingly bigger lesions, which will prevent chemotherapy, major surgery or worse in patients with increasingly dangerous tumors $[99,100,52]$. However, no commercially available automated flexible endoscope system exists to date [11].

Automated flexible endoscopy systems should be effective, cheap and easy to use. Especially this last requirement enables a more effective employment of less-trained personnel, for instance by performing endoscope insertion. A new system must not 
interrupt current clinical workflow significantly. If these requirements are not met, hospitals and doctors may never be persuaded to start using such a new system, and this is probably why no commercial systems are available yet.

We have a prototype system available that does meet many requirements. It can be used on every currently commercially available flexible endoscope system, which makes it easy to adopt $[32,33]$. The tip of the flexible endoscope is rotated by the motors on the turning knobs, while the user is still able to insert the shaft manually, ensuring user-friendliness. Controlling the motors can be done effectively using a joystick [53], but also (semi-)automatically using computer vision [46, 101, 102].

The premise of the current research is that the introduction of vision-based navigation in a robotized flexible endoscopy system can facilitate the use of such a system greatly. However, finding and testing a suitable navigation approach is not straightforward. In this chapter an approach is presented that in our opinion will navigate robotized endoscopes accurately and safely. This approach is an evolution of the work in [46]. The hypothesis about this navigation approach is tested in different experiments resembling flexible endoscopy procedures. The automated navigation results are compared to electromagnetic (EM) positioning and orientation data to evaluate the feasibility of this model.

\subsubsection{Related work in endoscope navigation}

Most research in image-based flexible endoscope navigation is focused on steering the tip of the endoscope. In general, the aim and approach of earlier research is to provide real-time, vision-based information for use in the closed-loop control of flexible endoscopes. The approach is predominantly focused on a technique called lumen centralization (overview provided in [83]). The medical term 'lumen' refers to the inside of a tubular organ structure. The deepest or darkest area on the inside of the human organ of interest is automatically detected and centered in previously described techniques. However, the tortuosity of the colon and the influence of manipulating an endoscope through it cause a highly unpredictable environment, in which the lumen is not always visible.

When observing endoscopic procedures in more detail it is seen that endoscope steering is not influenced by the distal tip alone. Insertion, retraction or rotation of the complete shaft can be applied by the endoscopist from outside the patient as well, as was pointed out by Chen et al. [103], among others. These external movements make automating endoscope navigation particularly challenging. Predictive organ modeling using preoperative images is an ineffective navigation approach for the same reasons (organ tortuosity, external disturbances).

Something that might be useful in endoscope navigation automation is information about camera motion, or egomotion of the camera. Endoscope images can be used to perform motion tracking of the camera, which is at the tip of the endoscope. Since it provides continuous updates on the environmental situation, no predictive modeling is required. If the previous path and/or camera pose are known, it becomes easier to correct the movements.

A well-known technique for egomotion estimation from images is optical flow (OF), 
used to estimate displacement between two images through pixel tracking $[83,79]$. This technique has been applied for medical use earlier. Mori et al. [59] use OF in human bronchoscopy images for camera position tracking. Subsequently, their goal is to use the endoscopic camera position to render the estimated virtual environment at that point. The environment estimation is performed using information from preprocedural CT images. The aim is to provide the endoscopist with a feature that is comparable to satellite navigation in a car: a road map for the procedure with respect to the current position. That navigation situation differs significantly from ours. The gastrointestinal tract is much more tortuous than the lungs. Pre-procedural images such as MR or CT images are of little use in our situation. The navigation situation for us more closely resembles a 'Simultaneous Localization and Mapping Problem' (SLAM) [104]. However, the tortuous (= dynamic) environment makes traditional SLAM approaches unfeasible. Instead, we focus on optimizing the visual feedback in the closed-loop control of a robotized flexible endoscope.

Mori et al. [59] used the focus of expansion (FOE) for direction estimation. In two consecutive images from a moving camera, the OF vectors (feature or pixel displacement vectors) may seem to diverge from a single point (see also Figure 3.3). This point is called the FOE. Mori et al. assume that the center of the smallest OF vectors is the FOE location. This may work for a perfectly tubular environment, but the configuration of the OF field is determined in general by objects within the field of view. Objects that are closer to the camera produce larger displacement vectors. In a colon for instance, folds can be closer to the camera and in this way influence the OF field. Small vectors merely indicate further away objects, and do not necessarily reflect the position of an FOE.

Bell et al. [84] used Artificial Neural Networks (ANN) and different OF algorithms to learn the camera pose from the optical flow between images of the gastrointestinal tract. They compared different illumination settings (white light and narrow band) and different image partitioning techniques (lumen-centered and grid-based) to determine the optimal algorithm. A major conclusion of their research is that optical flow-based camera pose estimation is a feasible technique. However, experiments were performed on a plastic model of the colon, which differs significantly in terms of image feature extraction from real tissue images. Our aim is to use a more generic approach for endoscope navigation and also test it using real tissue.

\subsubsection{Visual feedback in endoscope navigation}

To describe the endoscopic tip navigation, a local $(x, y, z)$-coordinate system with its origin at the endoscope tip will be used. Since the navigation is image-based, it is most natural to have this coordinate system coincide with the coordinate system of the camera (see also Figure 5.1). That is, the $z$-direction is pointing in the direction of the optical axis. If the camera is perfectly aligned within the endoscope, this direction equals the endoscope tip direction.

We may presume that the image provides sufficient information to find a 2D target position in the image. In fact, in the next chapters, we will demonstrate an accurate target detection method. This 2D image position implicitly defines the 3D target direc- 
tion relative to the body coordinate system. However, current tip motion also provides visual feedback for the robotic control loop. Therefore, we need a reliable motion estimation method for the endoscopic tip.

The motion of the tip is defined by a linear velocity vector describing translation and an angular velocity vector describing rotation. Both vectors are expressed in the local coordinate system of the tip. The direction of the translation vector is called the heading direction (Figure 5.2A). A full reconstruction of the path of the tip involves finding both the linear and angular velocity vectors from two consecutive frames. This information should then be used to control the tip such that a) the translational vector points towards the direction of the target (Figure 5.2B), and b) the tip direction also points in that direction.
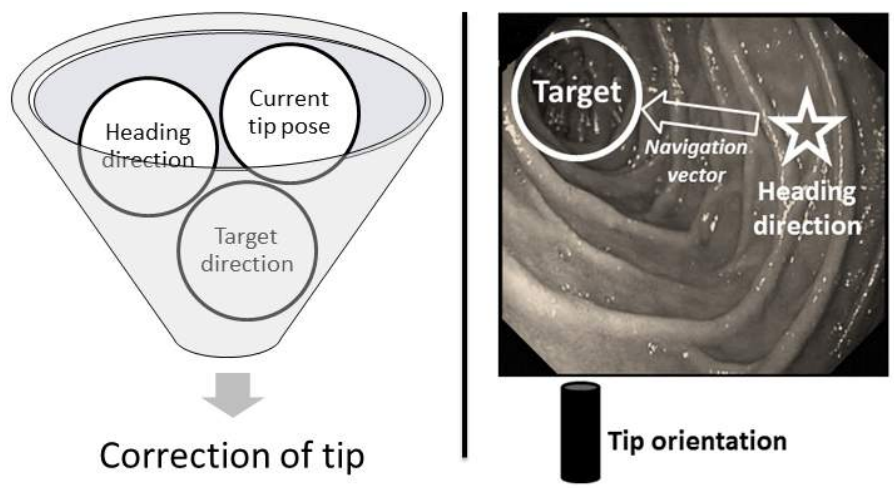

Figure 5.2: Schematic tip correction in automated flexible endoscope steering from the image point of view. The goal is to estimate camera heading direction $(\mathbf{A})$ and then correct this direction towards the target direction $(\mathbf{B})$.

In view of the limited controllability of the system, it is questionable whether goals a) and b) are attainable at the same time. Since the first goal is to reach the target, goal a) should be prioritized. If for simplicity's sake we neglect the angular motion, then finding the heading direction boils down to finding the FOE [46]. As said before, the FOE is a parameter resulting from the OF field. The FOE can also be regarded a derivation of the so-called epipolar geometry theory [73]. Under the constraint of a translational motion, the 2D FOE has the same coordinates in both images, and in epipolar geometry this point is called the epipole.

The idea of using epipolar geometry for analyzing the properties of the FOE is appealing since it means rotational motion estimation can be done. The 3D position of objects in a scene can be reconstructed from the epipolar geometry between images. Assuming that object positions are constant and the camera is moving, the displacement and the orientation of one camera pose relative to the other camera pose can be reconstructed from this geometry as well.

In the current research we use real tissue images to investigate a navigational 
model for flexible endoscope navigation based on the earlier used FOE [46]. The main question to answer is: how useful exactly are the FOE and the epipolar geometry in endoscopic images for flexible endoscope navigation?

\subsection{Methods}

In this section, first the theoretical model we used will be described. Next, the experimental setup and desired analyses are discussed.

\subsubsection{Model description}

The model for flexible endoscope navigation is based on the translation and rotation of the camera between two images. Let us regard the camera pose at time $t_{2}$ relative to the camera pose at time $t_{1}$. The vector ${ }^{1} \mathbf{t}_{2}$ is the translated position of the camera origin at $t_{2}$ expressed in the camera coordinate frame at $t_{1}$; the rotation matrix ${ }^{1} \mathbf{R}_{\mathbf{2}}$ contains the rotated base vectors of the camera coordinates at time $t_{2}$ expressed in the camera coordinates at $t_{1}$. For the estimation of camera motion, corresponding point features need to be found in the images taken at time $t_{1}$ and $t_{2}$. Assuming that the objects in the scene remain constant, the 3D camera displacement and rotation can be determined from the 2D displacements of these detected point features. This detection and localization is done in the images that the camera has made (OF calculation using the Lucas-Kanade method, see Chapter 4).

\section{The epipolar constraint}

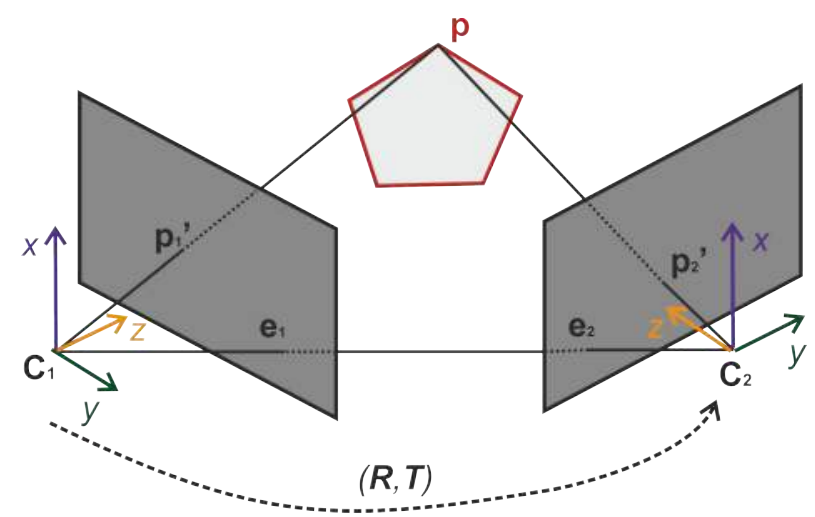

Figure 5.3: Representation of theoretical geometry between two images depicting the same object(s). $\mathbf{C}_{1}$ and $\mathbf{C}_{2}$ represent two camera coordinate frames. $p$ is a point on an object in the scene.

Consider two images of the same scene taken from two different camera origins, $\mathbf{C}_{\mathbf{1}}$

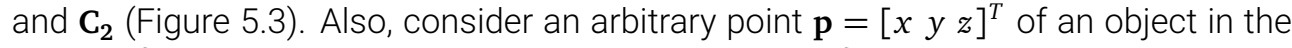
scene. If we represent this point $\mathbf{p}$ in the two camera frames by $\mathbf{p}_{\mathbf{1}}$ and $\mathbf{p}_{\mathbf{2}}$, then the 
following relation exists:

$$
\mathrm{p}_{2}={ }^{2} \mathrm{R}_{1}\left(\mathrm{p}_{1}-{ }^{1} \mathrm{t}_{2}\right)
$$

with ${ }^{2} \mathbf{R}_{\mathbf{1}}={ }^{1} \mathbf{R}_{\mathbf{2}}{ }^{T}$. As shown in Figure 5.3, the two camera origins $\mathbf{C}_{1}$ and $\mathbf{C}_{\mathbf{2}}$ define a $2 \mathrm{D}$ plane in the 3D space with the point $\mathbf{p}$. Therefore, the three vectors $\mathbf{p}_{1},{ }^{1} \mathbf{t}_{2}$, and $\mathbf{p}_{1}-{ }^{1} \mathbf{t}_{\mathbf{2}}$ are co-planar. As ${ }^{1} \mathbf{t}_{\mathbf{2}} \times \mathbf{p}_{\mathbf{1}}$ is a vector that is orthogonal to the plane, this co-planarity condition can be expressed as:

$$
\left(\mathbf{p}_{1}-{ }^{1} \mathbf{t}_{2}\right)^{T}\left({ }^{1} \mathbf{t}_{2} \times \mathbf{p}_{1}\right)=0
$$

Substitution of eq. 5.1 yields:

$$
\left({ }^{1} \mathbf{R}_{2} \mathbf{p}_{2}\right)^{T}\left({ }^{1} \mathbf{t}_{2} \times \mathbf{p}_{1}\right)=0
$$

When we define the matrix $\mathbf{T}$ :

$$
\mathbf{T}=\left[\begin{array}{ccc}
0 & -t_{x} & t_{y} \\
t_{z} & 0 & -t_{x} \\
-t_{y} & t_{x} & 0
\end{array}\right] \text { with }{ }^{1} \mathbf{t}_{2}=\left[\begin{array}{c}
t_{x} \\
t_{y} \\
t_{z}
\end{array}\right]
$$

the coplanarity condition can be rewritten in:

$$
\mathrm{p}_{2}^{T}\left({ }^{2} \mathbf{R}_{1} \mathbf{T}\right) \mathrm{p}_{1}=0
$$

or:

$$
\mathbf{p}_{\mathbf{2}}{ }^{T} \mathbf{E} \mathbf{p}_{\mathbf{1}}=0
$$

The matrix $\mathbf{E}={ }^{2} \mathbf{R}_{\mathbf{1}} \mathbf{T} \in \mathbb{R}^{3 \times 3}$ is called the essential matrix. Note that if $\mathbf{p}_{\mathbf{1}}^{\prime}$ and $\mathbf{p}_{2}^{\prime}$ are the projections of the point $\mathbf{p}$ on the two image planes (Figure 5.3), eq. 5.6 also holds for these projections:

$$
\mathbf{p}_{2}^{\prime T} \mathbf{E} \mathbf{p}_{1}^{\prime}=0
$$

By definition, the epipole is the image position at which the line between the two camera frame origins intersects that image (Figure 5.3). The line $\mathbf{l}_{1}$ between $\mathbf{p}_{1}^{\prime}$ and the epipole of the first image, $\mathbf{e}_{\mathbf{1}}$, is associated with the essential matrix $\mathbf{E}$ as follows:

$$
l_{1} \sim \mathrm{E}^{\mathrm{T}} \mathrm{p}_{2}^{\prime}
$$

where $\sim$ represents equality up to a scale factor.

In short, if a projection in one image is known, the projection in the other image must satisfy eq. 5.7. As such the essential matrix constrains the correspondence between the two projections. Moreover, the essential matrix encapsulates the relative position and orientation between the two positions from which the images were made. Therefore, if from a number of corresponding projections in the images (Figure 5.4) the essential matrix can be found, the first pose of the camera relative to the other pose can be deduced.

During endoscopy, multiple images are formed over time $t$ in an image plane parallel to the endoscope tip surface (Figure 5.4). If a fictitious point $\mathbf{p}=\left[\begin{array}{lll}x & y & z\end{array}\right]^{T}$ is 


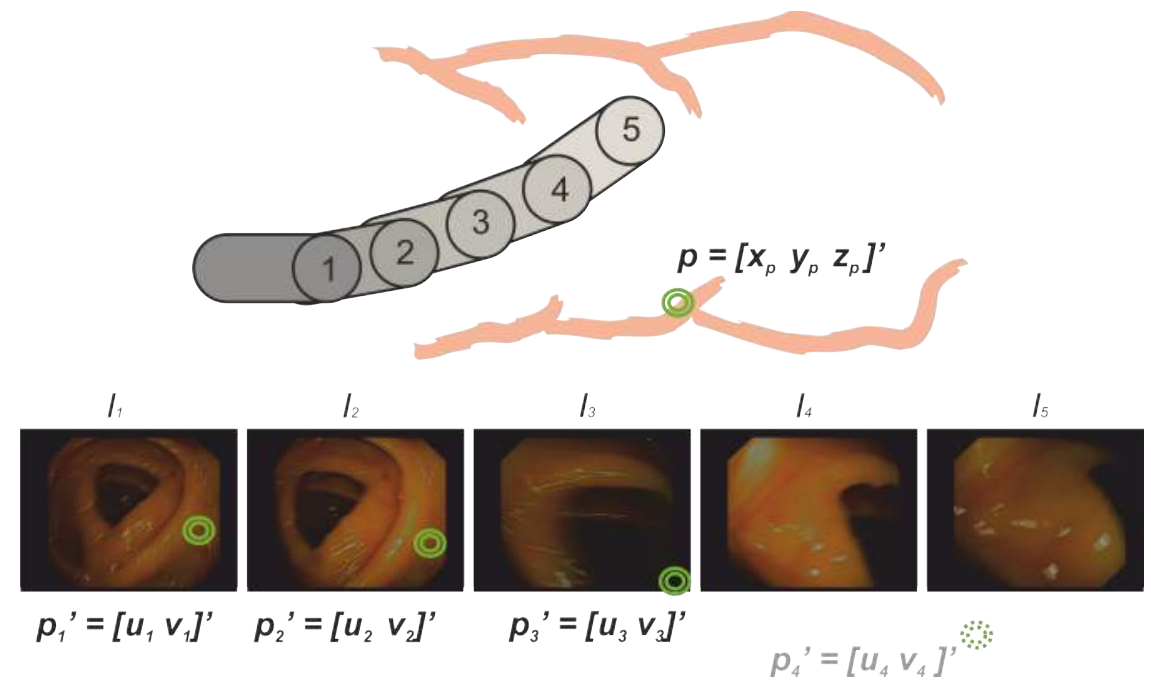

Figure 5.4: Schematic visualization of point feature displacement in images. In the top half, the colon side view with a forward moving endoscope is depicted. Point $\mathbf{p}$ is a point in the colon. When the endoscope moves, projected point $\mathbf{p}^{\prime}$ also shifts in the image. From multiple of these point displacements camera displacement can be deduced.

observed by an endoscope, the projection $\mathbf{p}_{\mathbf{t}}^{\prime}=\left[u_{t} v_{t} f\right]$ of this point in image $I_{t}$ can be tracked in the image $I_{t+1}$ (Figure 5.4). The displacement of the projected point between $I_{t}$ and $I_{t+1}$ is represented by a displacement vector $\mathbf{d}=\mathbf{p}_{\mathbf{t}}^{\prime}-\mathbf{p}_{\mathbf{t}+1}^{\prime}$. Detection and tracking of more 3D points will lead to a field of displacement vectors, or an OF field. These displacements are used in the epipolar constraint to solve the essential matrix (section 5.2.1).

\section{Finding the essential matrix}

Up to now the discussion was restricted to 3D camera coordinates. What we actually have are measurement results in pixel coordinates (row and column indices). Camera calibration is needed to connect the two coordinate systems (see Appendix A). In short, this means an object with known dimensions is recorded using a camera. From the images the object dimensions are estimated, too, and the discrepancy between the ground truth and the measurements can be attributed to camera properties. Lens distortion also influences the projection of 3D objects on a 2D image plane. The camera properties are stored in the camera matrix $\mathbf{K}$ and possible distortion factors are corrected for (for more information on the subject, see $[105,96,73])$.

The relation between $\mathbf{p}$ in real-world coordinates (denoted by ${ }^{w} \mathbf{p}$ ) and the projected $\mathbf{p}^{\prime}$ in pixel coordinates (denoted by ${ }^{c} \mathbf{u}$ to indicate different units) for $I_{t}$ is given by:

$$
{ }^{c} \mathbf{u}=\mathbf{K}\left[\begin{array}{ll}
\mathbf{I}_{3 \times 3} & \mathbf{0}_{3 \times 1}
\end{array}\right]{ }^{t} \mathbf{H}_{\mathbf{w}}{ }^{w} \mathbf{p}
$$

${ }^{w} \mathbf{p}$ and ${ }^{c} \mathbf{u}$ are both in homogeneous coordinates. ${ }^{c} \mathbf{H}_{w}$ is the rigid body transformation 
from the world frame to the camera frame. The intrinsic parameter matrix or calibration matrix $\mathbf{K}$ is built up as follows:

$$
\mathbf{K}=\left[\begin{array}{ccc}
f_{x} & s_{\theta} & o_{x} \\
0 & f_{y} & o_{y} \\
0 & 0 & 1
\end{array}\right]
$$

Here, $f_{x}$ and $f_{y}$ stand for the focal length expressed in pixel sizes in the $x$ - and $y$ direction. $o_{x}$ or $o_{y}$ are the $x$ - and $y$-coordinates (column and row indices) of the principal point in the image. Skewness of the pixel grid is described by the skew value $s_{\theta}$. Presuming that the grid is orthogonal, this value is often set to 0 .

The epipolar constraint can be cast into pixel coordinates by using the relation ${ }^{c} \mathbf{u}=$ $\mathbf{K}^{c} \mathbf{p}^{\prime}$, which means ${ }^{c} \mathbf{p}^{\prime}=\mathbf{K}^{-1} \mathbf{u}$. When substituting that in eq. 5.7 and generalizing time $\mathbf{1}$ to $t$ and time $\mathbf{2}$ to $t+1$ we obtain:

$$
\left(\mathbf{K}^{-1 c} \mathbf{u}_{\mathbf{t}+1}\right)^{T} \mathbf{E}\left(\mathbf{K}^{-1}{ }^{c} \mathbf{u}_{\mathbf{t}}\right)=0
$$

or:

$$
{ }^{c} \mathbf{u}_{\mathbf{t}+\mathbf{1}}{ }^{T} \mathbf{K}^{-T} \mathbf{E} \mathbf{K}^{-1}{ }^{c} \mathbf{u}_{\mathbf{t}}=0
$$

By introduction of the so-called fundamental matrix, $\mathbf{F}=\mathbf{K}^{-T} \mathbf{E} \mathbf{K}^{-1}$, as was previously used in Chapter 4, the constraint simplifies to:

$$
{ }^{c} \mathbf{u}_{\mathbf{t}+1}{ }^{T} \mathbf{F}^{c} \mathbf{u}_{\mathbf{t}}=0
$$

$\mathbf{F}$ can be computed from the displacement vectors of the OF field. Since $\mathbf{F}$ has 8 independent elements (homogeneous coordinates), 8 displacement vectors suffice to set up a system of linear equations to solve $\mathbf{F}$. Together with the relation $\mathbf{E}=\mathbf{K}^{T} \mathbf{F} \mathbf{K}$, this procedure gives us the essential matrix.

\section{Pose recovery}

Once the essential matrix is obtained, the question remains as to how to use this for camera pose estimation exactly. Skew symmetric matrices have the general property that following a singular value decomposition (SVD), two of their singular values are equal and the third equals zero [73]. Recall that $\mathbf{E}={ }^{\mathbf{t}+1} \mathbf{R}_{\mathbf{t}} \mathbf{T}$ (eqs. 5.5 and 5.6), with ${ }^{\mathbf{t}+1} \mathbf{R}_{\mathbf{t}}$ an orthonormal matrix and $\mathbf{T}$ a skew symmetric matrix. The singular values of $\mathbf{E}$ therefore have the described properties. Furthermore, since $\mathbf{E}$ is invariant under scaling by a constant, we scale $\mathbf{E}$ such that its nonzero singular values equal 1 . Hence, we can write the SVD as follows: $\mathbf{E}=\mathbf{U} \operatorname{diag}(1,1,0) \mathbf{V}^{T}$. Let us define a skew symmetric matrix $\overline{\mathbf{T}}$ and an orthonormal matrix $\overline{\mathbf{R}}$ as:

$$
\overline{\mathbf{T}}=\left[\begin{array}{ccc}
0 & -1 & 0 \\
1 & 0 & 0 \\
0 & 0 & 0
\end{array}\right] \quad \text { and } \quad \overline{\mathbf{R}}=\left[\begin{array}{ccc}
0 & 1 & 0 \\
-1 & 0 & 0 \\
0 & 0 & 1
\end{array}\right]
$$


it follows that $\overline{\mathbf{R}} \overline{\mathbf{T}}=\operatorname{diag}(1,1,0)$. Camera pose can then be recovered as follows:

$$
\begin{aligned}
& \mathbf{T}=\mathbf{V} \overline{\mathbf{T}} \mathbf{V}^{T} \text { and }{ }^{\mathbf{t}+\mathbf{1}} \mathbf{R}_{\mathbf{t}}=\mathbf{U} \overline{\mathbf{R}} \mathbf{V}^{T} \\
& \mathbf{T}=\mathbf{V} \overline{\mathbf{T}} \mathbf{V}^{T} \text { and }{ }^{\mathbf{t}+1} \mathbf{R}_{\mathbf{t}}=\mathbf{U} \overline{\mathbf{R}}^{T} \mathbf{V}^{T} \\
& \mathbf{T}=-\mathbf{V} \overline{\mathbf{T}} \mathbf{V}^{T} \text { and }{ }^{\mathbf{t}+1} \mathbf{R}_{\mathbf{t}}=\mathbf{U} \overline{\mathbf{R}} \mathbf{V}^{T} \\
& \mathbf{T}=-\mathbf{V} \overline{\mathbf{T}} \mathbf{V}^{T} \text { and }{ }^{\mathbf{t}+\mathbf{1}} \mathbf{R}_{\mathbf{t}}=\mathbf{U} \overline{\mathbf{R}}^{T} \mathbf{V}^{T}
\end{aligned}
$$

The translation vector ${ }^{\mathbf{t}} \mathbf{t}_{\mathbf{t}+\mathbf{1}}$ is easily extracted from $\mathbf{T}$ (see eq. 5.4). However, due to the scaling invariance of the essential matrix, the length of the translation vector remains unsolved and is set to unit length, i.e. $\left\|^{\mathbf{t}} \mathbf{t}_{\mathbf{t}+\mathbf{1}}\right\|=1$. Under this ambiguity, there are still four possible solutions as indicated in eq. 5.15. These ambiguities stem from the fact that from a mathematical point of view, the projection of a camera can occur from the front ( $F$ ) as well as from the back (B) of that camera. Hence, with two cameras, there are four possible solutions: FF, FB, BF, and BB. Physically, the only appropriate solution (FF) can be chosen by constraining the depth to be positive and the reconstructed points to be visible from the two camera view points.

\subsubsection{Experimental validation}

Main goal of the validation experiments was to compare the automatically computed displacement using the model described above to the ground truth obtained from electromagnetic $(E M)$ tracking measurements. Figure 5.5 depicts the complete experimental setup that was used for the validation experiments. The true camera positions are

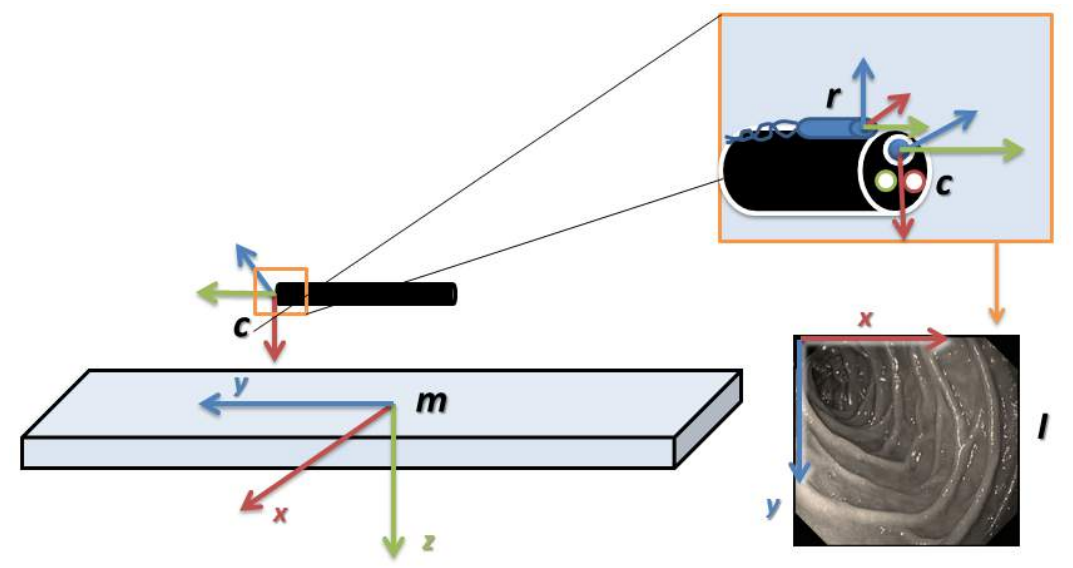

Figure 5.5: Experimental setup, schematic overview. The magnetic beacon ( $\mathbf{m}$ ) records the electromagnetic reference sensor position $(\mathbf{r})$. This sensor is attached to the camera $(\mathbf{c})$, which obtains the images $(I)$.

measured at the tip of the endoscope by $\mathbf{r}$. This sensor was manually attached to 
the endoscope. A rotation of a few degrees, or a translation of a few millimeters with regard to the camera lens could make a large difference in the final results. The computed displacements from the OF are expressed in the coordinate frame of the camera, which is why the sensor position needs to be expressed in the same frame.

The intrinsic camera properties are needed to estimate the relative position of the sensor (e) with respect to the camera (c) as well. The estimation can be obtained by computing the $4 \times 4$ homogeneous transformation matrix ${ }^{\mathrm{r}} \mathbf{H}_{\mathbf{c}}$ [106]. Transformation matrices between all coordinate frames were used to obtain this result, as displayed in Figure 5.6.

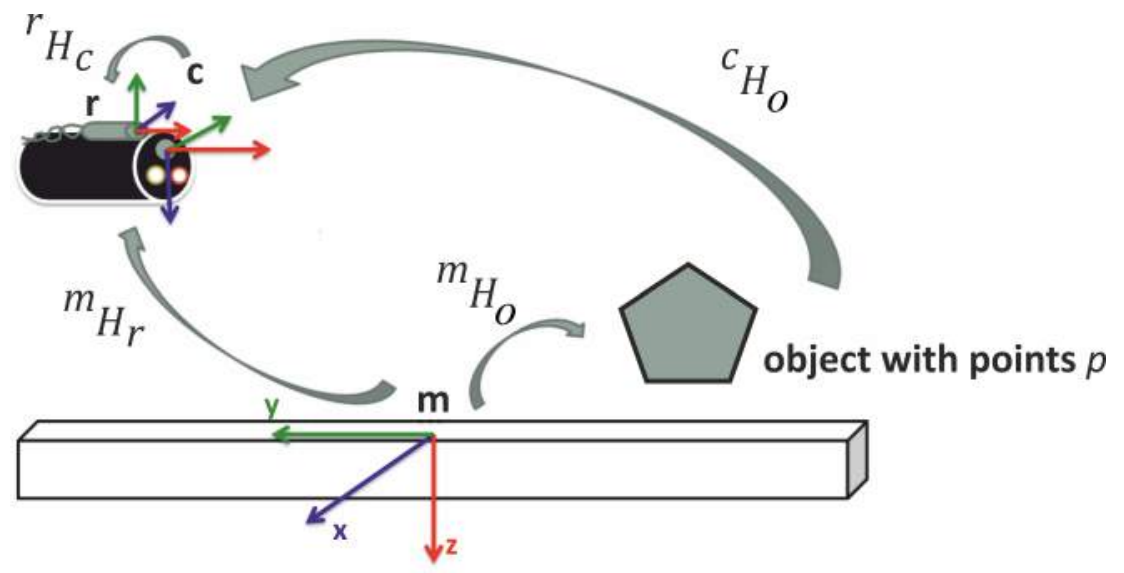

Figure 5.6: Transformation matrices and arrows to indicate the coordinate frame origin locations. The points $\mathbf{p}$ lie on the object $\mathbf{o}$, but are obtained from the image as $\mathbf{p}^{\prime}$, as described in section Model description.

Let ${ }^{\mathbf{m}} \mathbf{p}$ be the points of object $\mathbf{o}$ in 3D coordinates, expressed in coordinate frame $\mathbf{m}$ measured with respect to magnetic beacon $\mathbf{m}$. Assuming a rigid transformation, the matrix ${ }^{{ }^{m}} \mathbf{H}_{\mathbf{o}}$ can be used to describe the transformation of all points from within the object frame o to the magnetic beacon frame $\mathbf{m}$ :

$$
{ }^{m_{1}} \mathbf{H}_{\mathbf{o}}=\left[\begin{array}{cc}
\mathrm{m}^{\mathrm{R}} \mathbf{R}_{\mathrm{o}} & \mathrm{m}_{\mathbf{t}_{\mathrm{o}}} \\
\mathbf{0}_{3} & 1
\end{array}\right]
$$

in which ${ }^{\mathbf{m}} \mathbf{R}_{\mathbf{o}}$ is the $3 \times 3$ rotation matrix between the origins of frames $\mathbf{m}$ and $\mathbf{o}$, and $\mathbf{m}_{\mathbf{t}_{\mathbf{o}}}$ the $3 \times 1$ translation vector. The points in ${ }^{\mathbf{m}} \mathbf{p}$ are the features obtained from the OF algorithm, expressed in frame $m$ instead of the camera frame $c$, and can thus be used as a ground truth.

The position of the camera $\mathbf{c}$ is recorded with the reference sensor $\mathbf{r}$ (Figure 5.5). ${ }^{\mathbf{c}} \mathbf{R}_{\mathbf{o}}$ and ${ }^{{ }^{\mathbf{t}_{\mathbf{o}}}}$ represent the rotation matrix and translation vector of $\mathbf{c}$ with respect to $\mathbf{o}$, and are computed from the OF using the described model. These can in turn be used to compute ${ }^{{ }^{c} \mathbf{H}_{\mathbf{o}}}$ in a similar fashion as shown in eq. 5.16. However, the (fixed) transformation between the reference sensor and the camera is needed to compare the 
measured ground truth using $\mathbf{r}$ to the measured camera pose with the essential matrix. Therefore, during camera calibration, the positions of points ${ }^{\mathrm{m}} \mathbf{p}$ on the object $\mathbf{o}$ are measured once using an EM-tracked pointer, resulting in the matrix ${ }^{\circ} \mathbf{H}_{\mathbf{m}}={ }^{\mathbf{m}} \mathbf{H}_{\mathbf{o}}{ }^{T}$. The transformation matrix between the sensor frame $\mathbf{r}$, expressed in camera coordinates (c) is then computed from:

$$
{ }^{\mathrm{c}} \mathrm{H}_{\mathrm{r}}={ }^{\mathrm{c}} \mathrm{H}_{\mathrm{o}}{ }^{\mathrm{o}} \mathrm{H}_{\mathrm{m}}{ }^{\mathrm{m}} \mathrm{H}_{\mathrm{r}}
$$

The transformation matrix between $\mathbf{m}$ and $\mathbf{r},{ }^{{ }^{m}} \mathbf{H}_{\mathbf{r}}$, is obtained from the measured positions by $\mathbf{r}$.

${ }^{\mathrm{c}} \mathbf{H}_{\mathrm{r}}$ can now be used to express the measured ground truth in camera coordinates. As explained in the previous section, the projected point displacements are expressed in the camera frame as well to compute the camera pose ${ }^{\mathrm{c}} \mathbf{C}_{\mathrm{OF}}={ }^{\mathbf{t}+1} \mathbf{R}_{\mathbf{t}}{ }^{\mathbf{c}} \mathbf{p}+{ }^{\mathbf{t}+1} \mathbf{t}_{\mathbf{t}}$. This resulting pose is compared to the measurements from the EM tracker, which now also reflect camera pose since:

$$
{ }^{\mathrm{c}} \mathrm{C}_{\mathrm{EM}}={ }^{\mathrm{c}} \mathbf{R}_{\mathbf{r}}{ }^{\mathrm{c}} \mathbf{p}+{ }^{{ }^{c} \mathbf{t}_{\mathbf{r}}}
$$

If the navigation model is accurate, the following equation will hold:

$$
{ }^{\mathrm{c}} \mathrm{C}_{\mathrm{EM}} \triangleq{ }^{\mathrm{c}} \mathrm{C}_{\mathrm{OF}}
$$

It is expected that an error exists between the ground truth and the measurements, and that ${ }^{\mathrm{c}} \mathrm{C}_{\mathrm{EM}} \triangleq{ }^{\mathrm{c}} \mathrm{C}_{\mathrm{OF}}$ will not hold as such. The error of the pose estimation is therefore determined by the remaining rotation and translation between the measured camera pose of the EM system and the estimated camera pose by the epipolar geometry computation. These can be obtained through:

$$
\begin{aligned}
\Delta \mathbf{R}_{\mathrm{C}} & ={ }^{\mathrm{c}} \mathbf{R}_{\mathrm{EM}}{ }^{\mathrm{c}} \mathbf{R}_{\mathrm{OF}}^{\mathrm{T}} \\
\Delta \mathbf{T}_{\mathrm{C}} & ={ }^{\mathrm{c}} \mathbf{T}_{\mathrm{EM}}-\Delta \mathbf{R}_{\mathrm{C}}{ }^{\mathrm{c}} \mathbf{T}_{\mathrm{OF}}
\end{aligned}
$$

\section{Experimental setup}

The simulated colon environment either was a perspex tube (Figure 5.7), a plastic anatomical, a porcine or a bovine model (Figures 5.8 and 5.9). The perspex tube was chosen for the high level of controllability of the ground truth. If unexpected results would rise, an easy check could be done to see if the results were due to faulty measurements or had another cause. The plastic and animal bowel models were assessed for resemblance to the human gastrointestinal tract. The movements were performed by the same medical researcher for all experiments. All experiments started with the camera calibration (Figures 5.7A-B and 5.9A). For the perspex tube, the porcine model and the bovine model, we obtained the same displacements: straight forward, straight backwards, bending the tip up and down, bending the tip left and right, and rotating the endoscope along its shaft. To make sure the endoscope could be introduced properly, the tissue was fixated as shown in 5.9B, and insufflation was used.

\section{Materials}

For these experiments, an EM tracking system was used (NDI Aurora V2, Northern Digital Inc., Waterloo, Ontario, Canada) with a table-top (perspex setup) or a planar (tissue setup) field generator and a 6D reference sensor and 6D pointer. We were careful to 


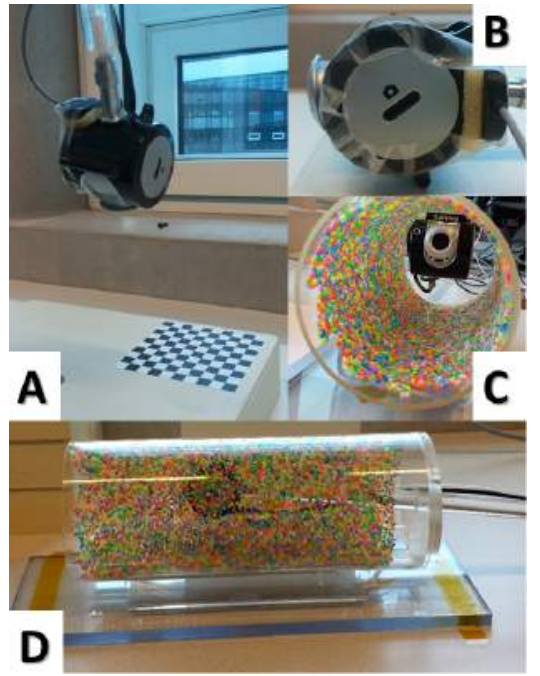

Figure 5.7: Experimental setup in which is shown how the equipment was positioned and how the measurements were obtained.

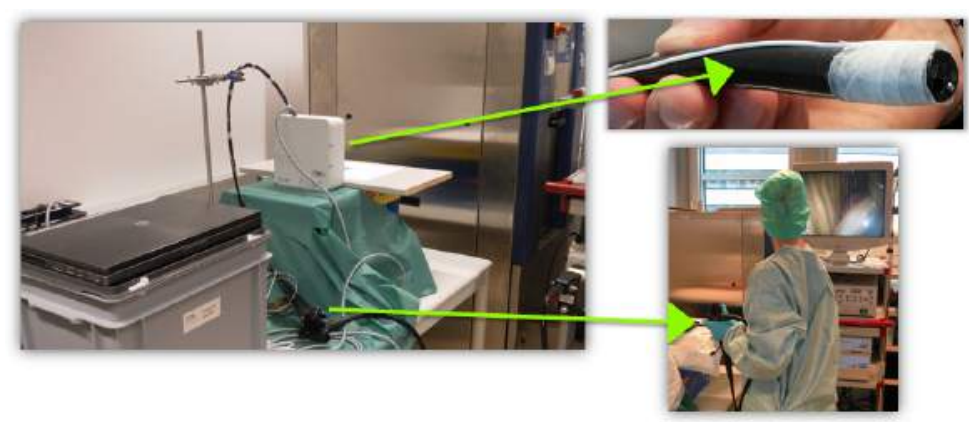

Figure 5.8: Experimental setup in which the equipment is shown. The measurements were obtained by a different person than the person performing the endoscopic movements.

use as little metal near the setups as possible (e.g. no metal frame under the table). For the perspex setup, the used camera was a Philips webcam SPC900nc/00 with PixelPlus. The perspex rod with the camera mount was custom-made. Note that in the picture still a metal rod was used as a camera mount, this was changed to perspex for the reported experiments. The used endoscope was a standard Olympus CF180 colonoscope connected to an Olympus CV-180 Evis Exera II video processor. A standard laptop was used for the analyses (HP Elitebook 8560w, 64 bit Windows 7, 4GB DDR3 RAM) with MATLAB 2014b (The Mathworks Inc., Natick, MA, USA). The used an- 


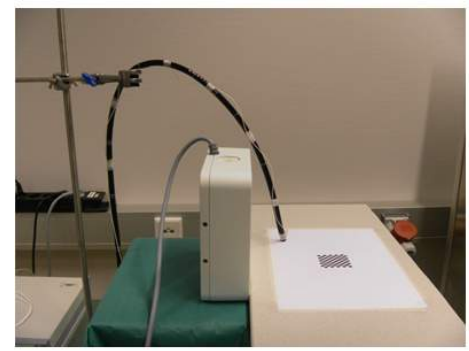

A

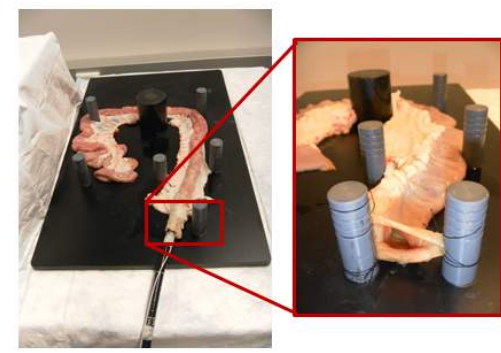

B

Figure 5.9: Experimental setup for the camera calibration with the checker board (A). In $\mathbf{B}$, the fixation of the colon model and the endoscope position is shown.

imal bowel tissues were obtained following both ethical and sanitary standards and laws in the Netherlands.

\subsection{Results}

The calibration procedure was successful in all experiments (Figure 5.10). Computa-

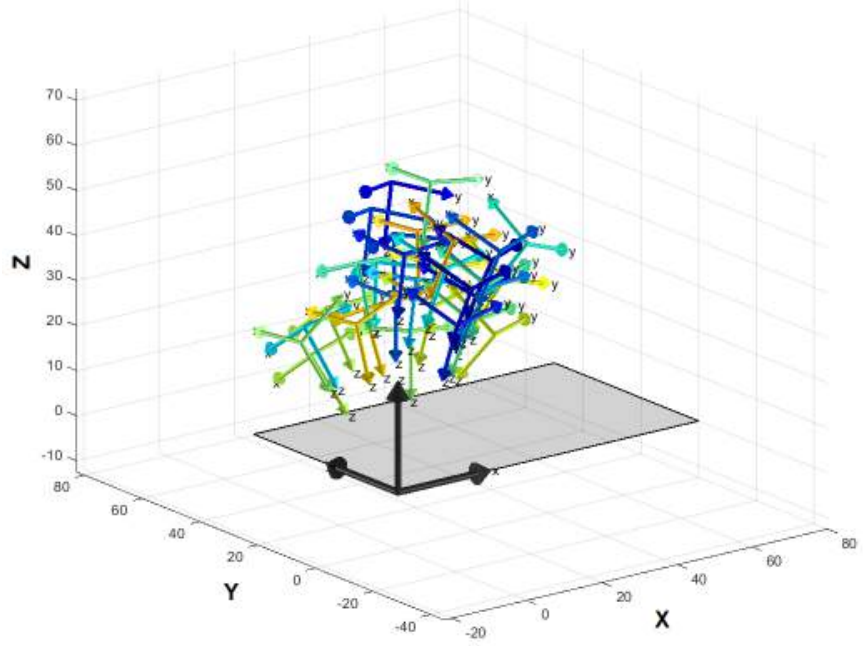

Figure 5.10: The coordinate frames of the cameras at all positions where calibration images were taken. Frame positions are reprojected in the world frame. Note that the cameras point with the $z$-axis, the axis we defined as the optical axis, to the checker board. 
tion of ${ }^{c} \mathbf{H}_{\mathbf{r}}$ succeeded with a mean translational and angular error of $6.2 \mathrm{~mm}$ (root mean square error) and 8 degrees, respectively.

The performed motions were well-discernible from the EM tracking data (Figure 5.11). When a straight path was performed, the EM tracking showed a long, stretchedout path. The apparent bends in the path are small; the $x$ and $y$ axes ranges are 2-5 $\mathrm{cm}$.
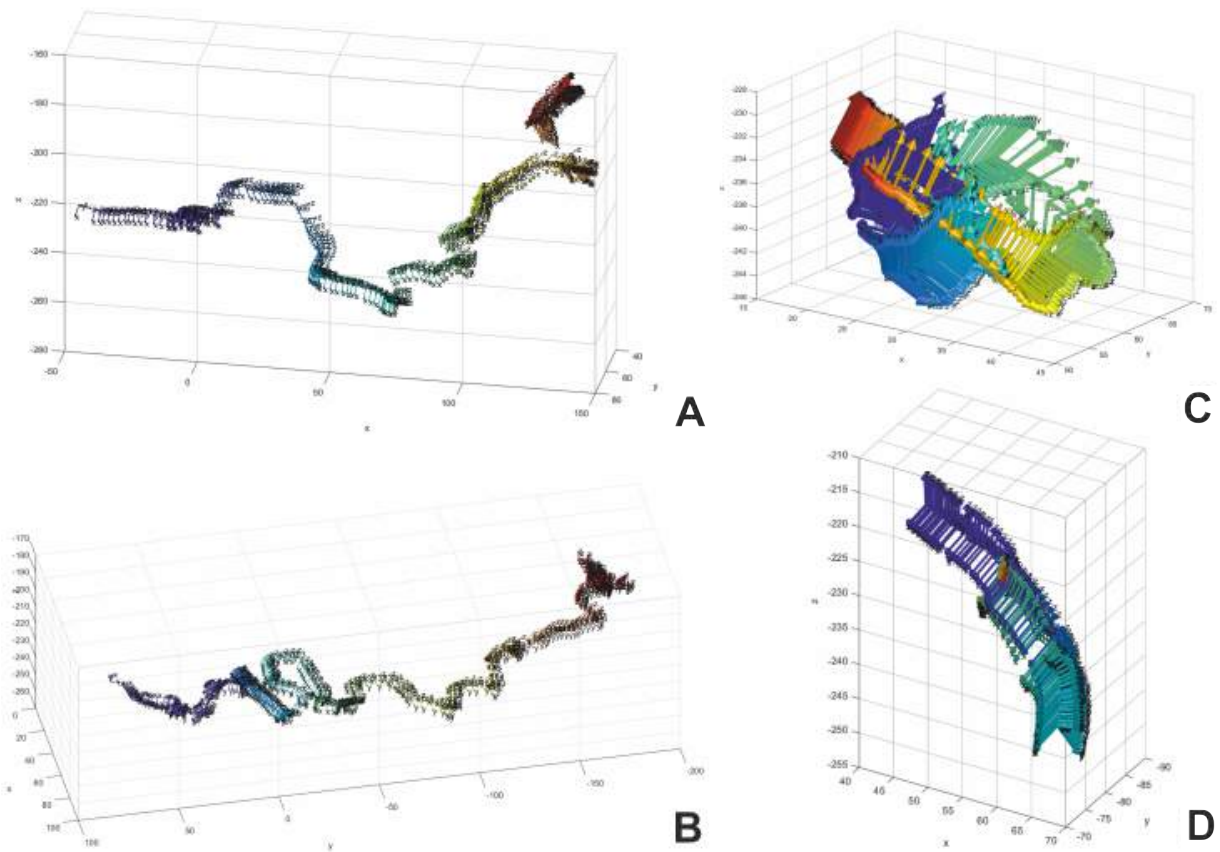

Figure 5.11: The motion path of the endoscope is well-discernible from the EM tracking data. The axis labels are too small, but it is the general motion that is important here. Figures $\mathbf{A}$ and

B show the straight paths in porcine and bovine tissue, respectively. $\mathbf{C}$ shows a rotational motion along the z-axis, in $\mathbf{D}$ the endoscope is looking up and down.

Optical flow could be estimated in all different environments except for the anatomical model (Figure 5.12). This was due to a lack of texture in the images. FOE and epipolar geometry subsequently could not be estimated from that model.

A FOE computation alone led to inconsistent results (Figure 5.13). We expected a smooth signal that would correspond to the EM outcome. Instead, the outcome is noisy and the FOE 'shoots' over the images. This was consistent over all the modalities.

The epipolar geometry computation was performed on a perspex tube measurements, because these measurements showed most consistent. In addition, the ground truth motion was very well-controllable. A measurement that only produced a smooth translation forward (z-axis), and no rotation was analyzed (Figure 5.14) What can be 


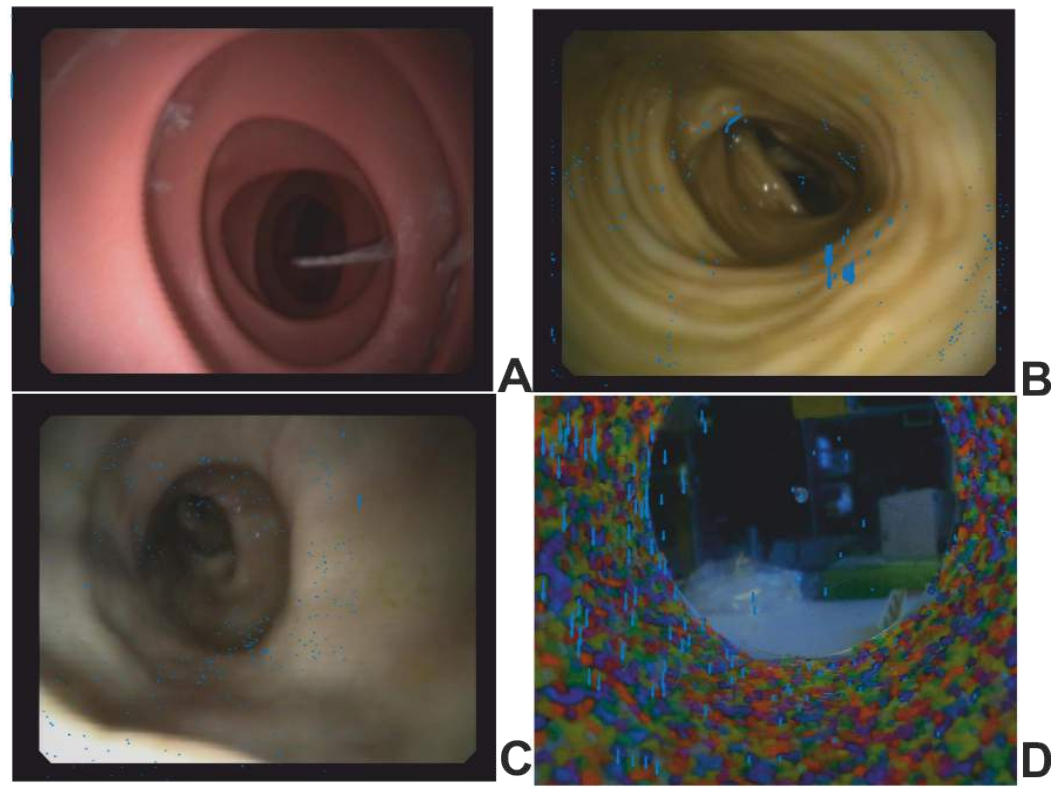

Figure 5.12: Example results of all environment modalities. OF vectors are depicted as blue arrows. Note that in the plastic anatomical model (A) no vectors were found except along the left edge. Images $\mathbf{B}, \mathbf{C}$ and $\mathbf{D}$ depict the bovine, porcine and perspex models.

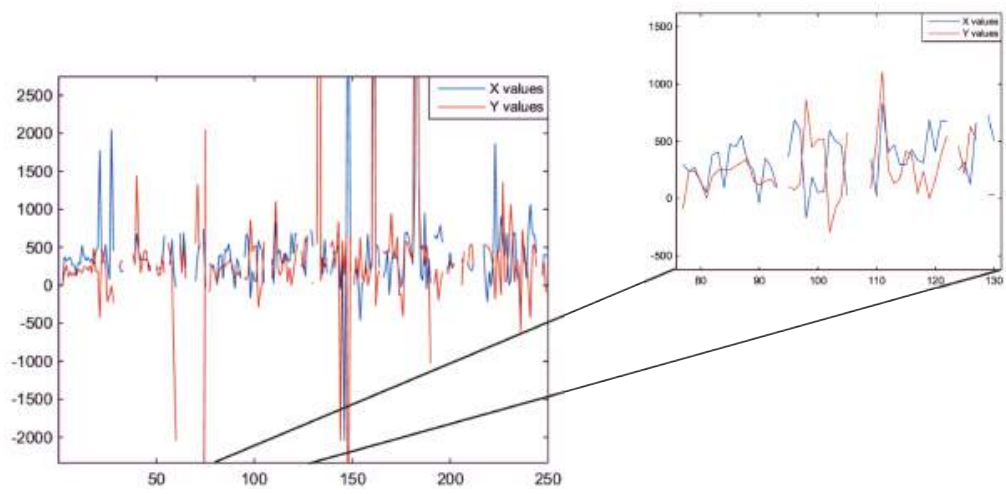

Figure 5.13: Example result of an X,Y FOE graph in pixels (straight measurement, porcine model). It can be seen that the values differ greatly between images (zoomed part). In addition, large outliers are present. This can be explained by a parallel flow field, which would cause the FOE to adopt coordinates that are infinite. The missing values are caused by OF results from which a FOE could not be estimated. 
seen from the figure is that especially the rotation results suffer from the same noisy measurements as the FOE results. The rotation matrix we expected would show no rotation, i.e. the a $3 \times 3$ identity matrix. The variance in rotation results for the visual estimation is again high. The environment modality (plastic, porcine, bovine) did not make a difference in this outcome. Since it is obvious from the images that the ground truth does not resemble the estimation, we have not performed an exact error computation.
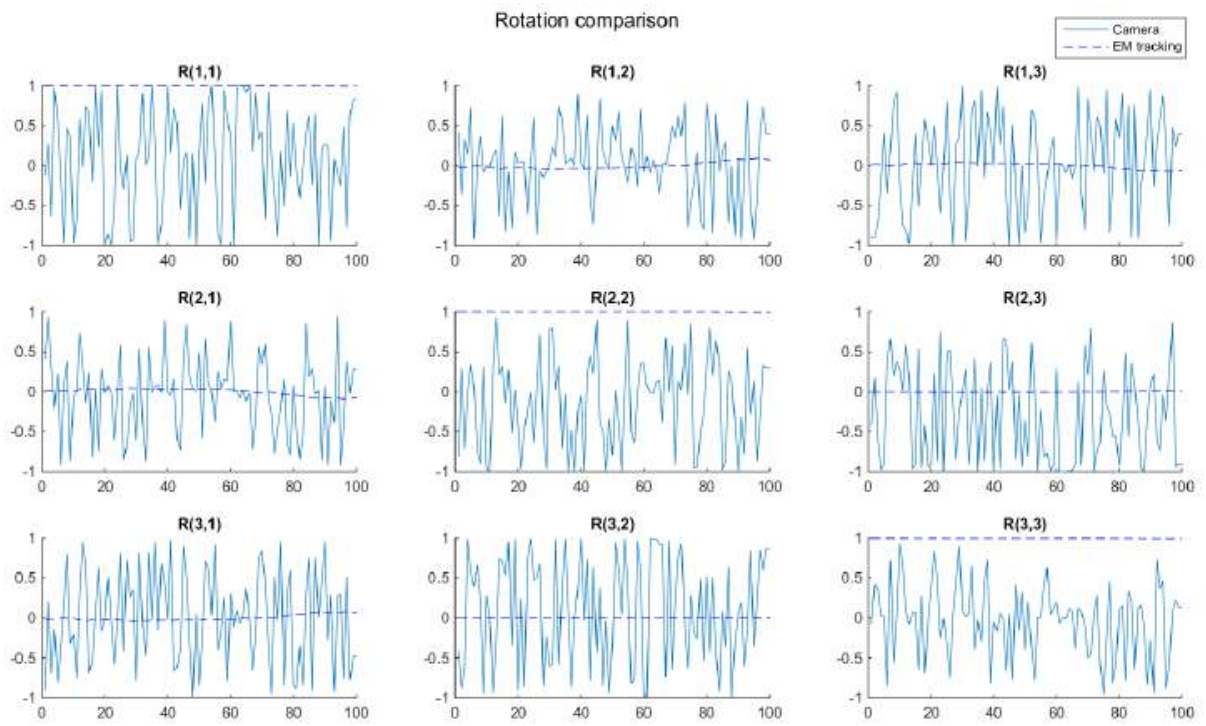

Figure 5.14: Example result of a translational measurement in the perspex tube. Each graph shows the value of the respective element in the rotation matrix. Note that the EM results indeed show no rotation, but that the visual results again show high variance.

\subsection{Discussion}

This chapter was a continuance of the work in Chapters 3 and 4 . The goal was to determine camera motion from endoscopic images. Earlier, we have used the FOE as a measure for this in a feasibility study. The FOE is a derived measure from the epipolar geometry between images. Using this geometry, the 3D displacement of objects in a scene can be reconstructed. Interestingly, when assuming that object positions are constant and the camera is moving, camera pose can be reconstructed from this geometry as well. The measurements were performed on real tissue to produce the most realistic images in terms of texture, which is an important image feature for OF computation. However, we also used a perspex tube and a plastic anatomic model to be able to explain possible deviations from our expectations. 
The porcine model was shown to resemble the human gastrointestinal tract best. The plastic anatomical model lacked texture, an image feature that is vital to compute camera motion from images. We would therefore recommend to always use real animal tissue if a vision algorithm ultimately needs to work on tissue images.

The camera calibration procedure and the EM data analysis performed as expected. The endoscope motion could be well-reconstructed from the measurements. However, the camera motion that was estimated from the images - either by FOE or epipolar geometry - did not represent the true motion. We have identified two possible causes for these results:

1. The speed of the endoscope in combination with the vicinity of the objects in the scene caused more motion blur effects than anticipated and therefore an inaccurate optical flow measurement.

2. A heading direction estimation as we proposed here can only be done between two camera frames, which leads to a maximum of 24 measurements per second. The visual measurements were probably undersampled.

Motion blur causes the projections of objects in the image to smooth out. Since the assumption is that the environment is locally static and camera motion causes the optical flow, the estimation of the environment needs to be performed accurately. The smoothing of objects in an image leads to extreme differences between two images, causing the visual tracking to fail.

Even if the motion blur effects could be alleviated, even in manual FOE results large shifts occur ([46]). To use information on camera motion or pose in a robotic feedback loop, more measurements per second will benefit the stability of the system and a higher number of estimations is therefore recommended.

In addition, the computational cost of the current approach is relatively high. On average, one frame took 6.5 seconds to compute. It is possible to make this system work in real-time, but this will also increase system costs. Great care should then be taken to still comply with the requirements for a clinical endoscopic navigation system that were named in the Introduction.

\subsection{Conclusion}

The aim of this research was to investigate the feasibility of the FOE or epipolar geometry reconstruction as a measure for endoscope heading direction estimation. The resulting motion estimation from the camera suffers from a high variance. In addition, the computational time was questionable. At this point, the described approach is deemed unsuitable for application in a robotized endoscope system. However, imageguided endoscope navigation in itself is a valid idea, which will be shown in the next chapters of this thesis.

\subsection{References}

Please refer to the bibliography at the end of this thesis. 


\section{Validation of an automated lumen detection algorithm}

John F. Kennedy

Published as: N. van der Stap, C.H. Slump, I.A.M.J. Broeders, \& F. van der Heijden (2014). Image-based navigation for a robotized flexible endoscope. In: 1st Int. Workshop on CARE, 14-18 Sept 2014, Boston, MA, USA. pp. 77-87. Lecture Notes in Computer Science 8899. Springer International Publishing. ISSN 0302-9743. ISBN 9783-319-13409-3.

Reprinted with permission.

Especially during high-throughput procedures, such as a colonoscopy, image-based navigation support algorithms could improve procedure turnaround and ergonomics for the endoscopist. In this study, we have developed and implemented a navigation algorithm that is based on image classification followed by dark region segmentation. Robustness and accuracy were evaluated on real images obtained from human colonoscopy exams. The developed target detection algorithm provided accurate results and is thought to provide reliable assistance in the clinic. 


\subsection{Introduction}

Robotizing flexible endoscopes has potential advantages for routine medical diagnostic as well as for complex therapeutic interventions [107]. Flexible endoscopes are 1.8m long flexible tubes, controlled by steering knobs and with a lens system and CCD or CMOS chip in the tip. This configuration makes them suitable for minimally invasive procedures. Demand for these kinds of procedures is growing with the trends of more preventive medicine and of reducing the amount of scarring due to surgical procedures. The design of flexible endoscopes has sufficed for decades, but the increased demand now leads to a need for more efficient procedures in terms of turnaround. Additionally, one instrument with two Degrees of Freedom (DOF) that protrudes from the tip does not meet the dexterity that is required for complex interventions. Control of the endoscope is far from intuitive; high physical workload and injuries among endoscopists have been reported (e.g. [23]). A telemanipulation system for current

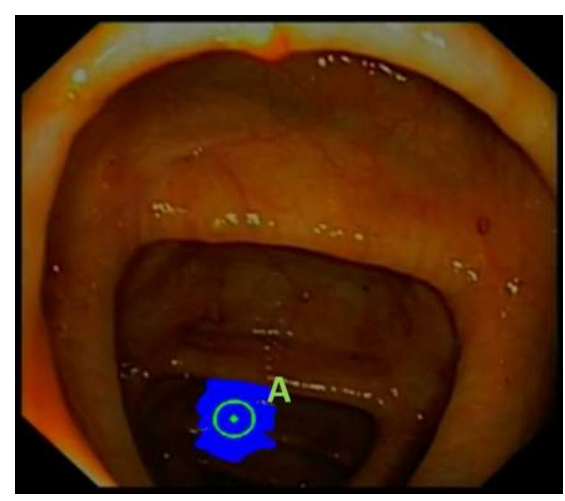

Figure 6.1: Example of dark region segmentation (target direction determination) in a real colonoscopy image.

flexible endoscopes has been developed [53]. This system, called TeleFlex, aims at improving procedure efficiency and reducing endoscopist workload at relatively low cost. Image-based navigation and control of the robotic system may help to improve the intuitiveness of the system and reduce procedure time [83]. During routine diagnostic 'high-throughput' procedures, specifically colonoscopies (endoscopy of the colon), the endoscope is first fully inserted in the colon and then retracted slowly to interpret possible lesions. The retraction phase has a recommended duration of six minutes [19] while the mean insertion phase of a colonoscope is 10 to 20 minutes [17], so efficiency improvement can be achieved during the insertion phase.

Centralization of the target direction by automatic endoscope tip correction is thought to lead to a smooth insertion (e.g. [28]), but this application has never been brought into clinical practice. The target direction in images of the colon can easily be found by segmentation of the darkest region in the image (Figure 6.1, point A). This region in most cases represents the deepest part of the inside of the organ (lumen) and thus 
corresponds to the target direction for steering. The center of this region will be called 'target' from now on.

A problem with image-based navigation in colonoscopy is that the lumen often is not visible. This situation occurs when the tip of the endoscope is (almost) touching the wall of the colon, a so-called 'red-out'. In addition, fluids like blood or water frequently occlude the field of view and influence image analysis. Bubbles and motion blur are other artifacts (see subsection 6.2.1) that occur often during these kinds of exams.

At our department an attempt was made to develop image-based endoscope navigation earlier [32]. The center of gravity (CoG) of the image was used as the target area towards which the endoscope tip was steered. While the algorithm performs well on a plastic model of the esophagus and stomach, in real colonoscopic image sequences it suffers from the artifacts mentioned above and it drifts quickly.

Probably one of the main reasons automated navigation algorithms for colonoscopy are currently not working in a clinical setting is that the colon is too unpredictable for complete autonomous navigation. During colonoscopy, the colon is insufflated with air, which changes the diameter and folds of the colon significantly. In addition, the endoscope itself causes a considerable shape change of the colon, limiting the use of pre-procedural imaging information (e.g. CT images). Therefore, we have chosen for a semi-autonomous approach of endoscope navigation and have designed and realized a support system. The navigation algorithm that was developed for this system was tested on real colonoscopy images. Main requirements for such an algorithm are that it should be able to perform robustly, accurately and in real-time in clinical practice. The focus in our design lies on clinical applicability. Goal of this study is to evaluate the accuracy and robustness of the designed algorithm.

\subsection{Materials and methods}

A target centralization algorithm was designed for and implemented in a robotized flexible endoscopy system. Two main requirements were imposed on system functionality. First, the complete system needs to work in real-time without significant hardware requirements to become applicable in a clinical setting. Second, the endoscopist needs to be able to overrule the algorithm instantaneously and the complete functionality needs to be intuitive.

In the case of Teleflex, control of the system (Figure 6.2) can either be established through remote user input (e.g. joystick or touch pad device), or through the imagebased navigation algorithm. This algorithm's main task is to detect the target of the endoscope through image analysis. The navigation algorithm should not be influenced by frequently occurring artifacts like lumen occlusion, fluids on the lens or bubbles in the field of view.

In this section, first the system will be described in more detail (subsections 6.2.1 and 6.2.2), and then the workings of the algorithm (subsection 6.2.3). Subsection 6.2.4 contains the details of the analyses we have performed. 


\subsubsection{Materials}

Endoscope systems typically produce RGB image sequences ranging from 25 to 30 frames per second of varying resolution, depending on vendor and model. In the case of this experiment, an Olympus CF180 colonoscope was used to record 576x768 pixel images with 25 frames per second. This endoscope has a field of view of 170 degrees and a field depth of 3 to $100 \mathrm{~mm}$. An endoscope can be advanced up to tens of $\mathrm{mms}$ per frame in some areas of the colon, making motion blur artifacts in images relatively common.

\subsubsection{System design}

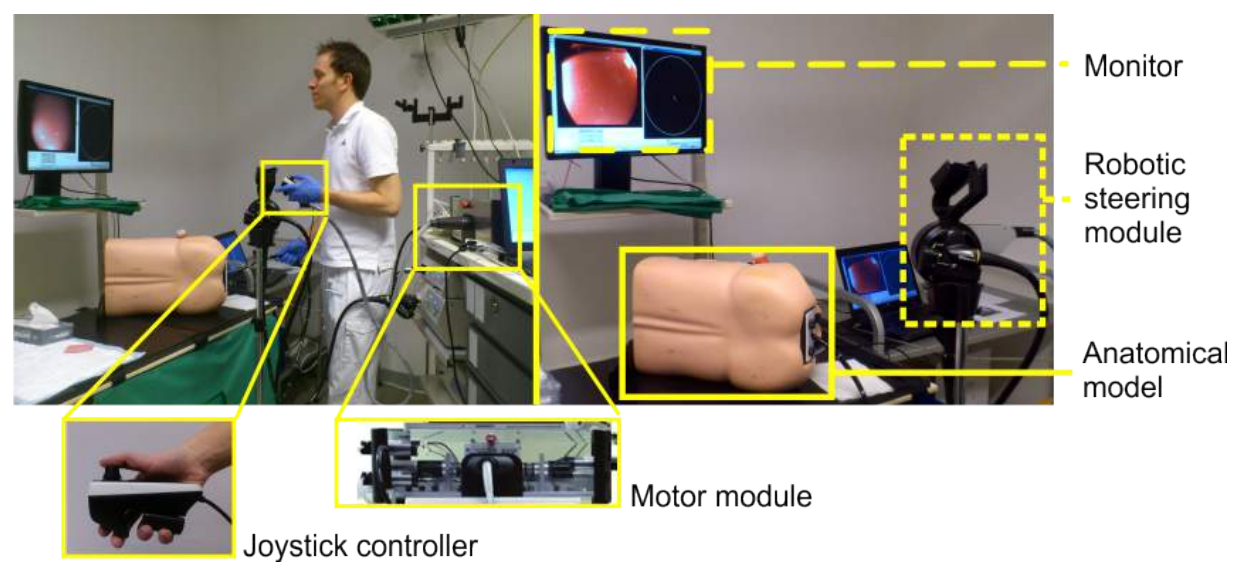

Figure 6.2: Overview of the experimental setup. Left: an endoscopist working with the system using a joystick controller. The navigation algorithm is enabled with a button press. The anatomical model is used to represent a patient. The robotic steering module is clicked on a conventional flexible endoscope and motors in the motor module turn the endoscopic steering knobs, using input from the joystick or the navigation algorithm.

The complete system setup and control is displayed in Figures 6.2 and 6.3. Only the tip of the endoscope is controlled by the steering knobs, and consequently the robotized system only influences tip motion in the currently used setup. The computer that is used for navigation and control is a standard laptop.

The user can enable the automated steering by keeping a button on the interface pressed. If the button is pressed, the navigation algorithm computes a steer command by finding the target direction. The algorithm can be overruled by using the joystick or by releasing the button. If the algorithm is enabled, the system attempts to centralize the target, i.e. turn the motors in the motor unit to rotate the endoscope's steering knobs until the tip is in the correct position. However, constraints are imposed in the movements of the motors to prevent instability of the system. Therefore it is possible that the tip position is not reached before the next target location update (analysis 


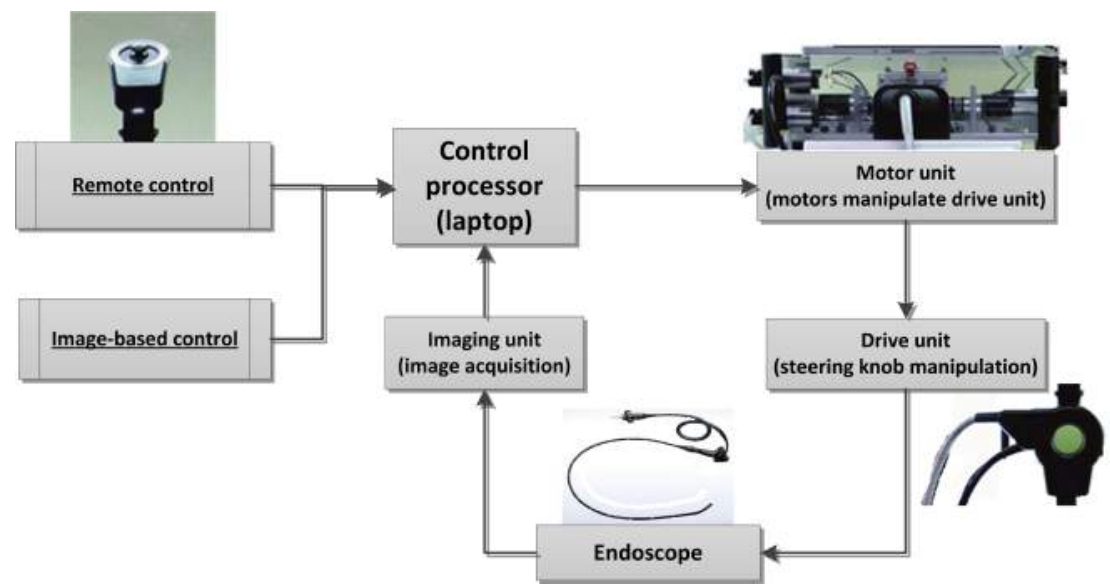

Figure 6.3: Flowchart of components in the robotized endoscopy system. Two types of control input can be discerned: remote (user) controlled steering and image-based steering. The motor unit controls the endoscopic steering knobs through the drive unit that is attached at the endoscope handle. Images are acquired from the video processor of the endoscope system.

of the next image). Nonetheless, by using previous, reliable information, the target ultimately will be reached with the navigation algorithm.

\subsubsection{Navigation algorithm}

Detection and correction of all possible artifacts separately is thought to lead to considerable computational effort and to still not lead to a robust solution for endoscope navigation because of the unpredictable environment. However, not in every frame a new target needs to be determined. Directional changes during colonoscopy occur gradually, which means previous accurate information can be used in the current frame. Yet, determining accuracy of target direction estimation automatically is not straightforward. Dark region segmentation leads to a large spread in results for many possible accuracy features. Therefore, we have designed an image classifier based on image variance and entropy that will predict whether a frame contains reliable information and thus whether the segmentation can be expected to be reliable (Figure 6.4). If it is expected that the information is unreliable, the algorithm will re-initialize by computing the Center of Gravity ( $\mathrm{CoG}$ ) and using this as input location for the Gauss filter.

Let $i$ be the frame index of an image sequence $f_{i}(\mathbf{x})$ with $\mathbf{x}$ the 2D pixel positions, and let $\mathbf{y}(i)$ be the 2D position of the center of the lumen. $\hat{\mathbf{y}}(i)$ is the estimate of $\mathbf{y}(i)$. The algorithm is initialized in the first frame of the sequence $(i=1)$ by the CoG computation described in [32]. This provides the estimate $\hat{\mathbf{y}}(1)$. All other frames of the sequence are processed according to: 


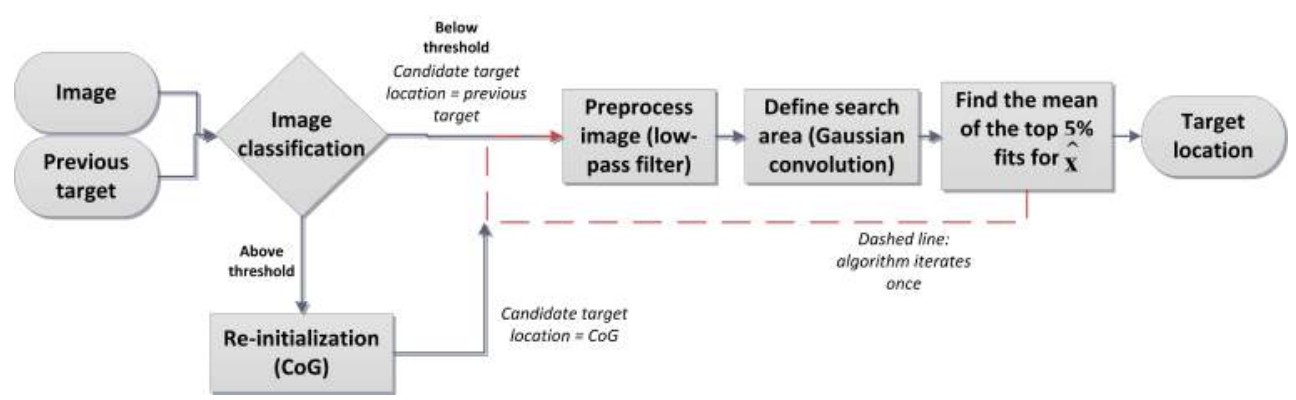

Figure 6.4: Stepwise approach of the algorithm with an image and the previous target as inputs. If the image is ruled 'uninformative', it does not resemble the previous frame at all. Therefore, previous target information is then discarded and the COG is recomputed. This location will then be used as candidate location for the target.

1. Invert and low-pass filter the frame:

$$
g(\mathbf{x})=\left(1-f_{i}(\mathbf{x})\right) * h(\mathbf{x})
$$

2. Window the result at the previously found position:

$$
g_{\text {win }}(\mathbf{x})=w(\mathbf{x}-\mathbf{y}(i)) g(\mathbf{x})
$$

3. Estimate the lumen at the position of the global maximum:

$$
\hat{\mathbf{y}}(i)=\arg \max g_{\text {win }}(\mathbf{x})
$$

Gaussian low pass filtering is applied to suppress the influence of noise. The inversion of the frame turns the search for a minimum into a search for a maximum. The window function is a Gaussian with the peak located at the previously estimated lumen position. So, it restricts the search area to a vicinity of that previous position, and also biases the current estimate towards this previous one. If a frame is classified as 'uninformative' (see below), the sequence is regarded as terminated, and the algorithm is re-initialized with the following frame.

\section{Image classifier}

Rationale behind the image classifier is that wall view frames, or red-outs, contain the least information. These frames are recognized from three features: image variance after high- and low-pass filtering and image entropy. We have established a training data set consisting of two classes: wall view images and other images. These images were obtained from colonoscopy exams in several patients. The classifier was then designed as follows. Suppose the data is organized in a three-dimensional (3D) vector z: high-pass filtered variance, low-pass filtered variance and entropy of two classes, $\omega_{1}$ and $\omega_{2}$, Differentiation between the classes occurs by transforming the data into a log-likelihood ratio $\Lambda$, defined as:

$$
\Lambda=\log \frac{p\left(\mathbf{z} \mid \omega_{1}\right)}{p\left(\mathbf{z} \mid \omega_{2}\right)}
$$


with $p\left(\mathbf{z} \mid \omega_{k}\right)$ the likelihood function [64]. Under the assumptions of normally distributed data sets with equal covariance matrices, $\Lambda$ becomes a linear mapping of $\mathbf{z}$ :

$$
\Lambda=\mathbf{a}^{T} \mathbf{z}+b
$$

The likelihood ratio $\Lambda$, conditioned on the two classes, are two Gaussians with equal variance $V$ and means $\mu_{1}=-\frac{1}{2} V$ and $\mu_{2}=\frac{1}{2} V$, respectively. If the densities are normalized with respect to variance, the distance between the means becomes $\frac{\mu_{2}-\mu_{1}}{\sqrt{V}}=$ $\frac{V}{\sqrt{V}}=\sqrt{V}$. This normalized distance is called the discrimination of the classifier. A value above 4 makes the classifier reliable [64].

\subsubsection{Experiments}

For the complete algorithm to work properly, the classifier had to be trained first. 265 images of colonoscopy exams were collected; 172 were labeled in class uninformative and the remaining 93 were labeled informative. A Linear Bayes Normal Classifier mapping was applied from the training set using the 'PRtools' toolbox [108] in Matlab R2011b (The Mathworks Inc., Natick, MA, USA). The mean values of the three features for both classes, $3 \times 1$ vectors $\mathbf{m}_{1}$ and $\mathbf{m}_{2}$, and the covariance $C$ result from this mapping. For the log likelihood ratio $\Lambda$ then:

$$
\mathbf{a}=\mathbf{C}^{-1}\left(\mathbf{m}_{1}-\mathbf{m}_{2}\right) \text {, and } b=-\frac{1}{2}\left(\mathbf{m}_{1}+\mathbf{m}_{2}\right)^{\prime} \mathbf{a} .
$$

After the classifier was trained successfully, it was implemented in the algorithm. Next, the algorithm was tested for robustness on five image sequences from human colonoscopy exams. In Table 6.1, properties of the test image sequences are listed. We have categorized the sequences in Easy, Medium or Hard. We expect the sequence of category 'Hard' to provide poorer results than the sequence of category 'Easy'. For Medium (Hard) better results are expected than for (Medium) Hard.

For each sequence, the steering target was manually annotated per frame. This target was defined as 'the point to which a human user would steer the endoscope'. The algorithm resulted in several measurement parameters for each sequence. The target location was detected and compared to the manual results using intra-class correlation analysis (ICC, $[45,67])$. The ICC is the main measure to determine accuracy of the algorithm. Additionally, the number of useful frames according to our classifier provides information on the quality of the frames in a sequence. This is expected to clarify whether a poor result in target direction over a sequence is due to frame quality or has another cause and therefore provides information on algorithm robustness as well.

\subsection{Results}

The best result was obtained with $\sigma$, the peak width of the Gaussian window function, set at 3. The discrimination of our classifier then was 6.8 using all three features, making it excellent. Because of the excellent result a feature reduction was attempted. 


\begin{tabular}{c||lcl} 
Sequence & Artifact description & No. of frames & Category \\
\hline $\mathbf{1}$ & No artifacts, clean bowel & 77 & Easy \\
$\mathbf{2}$ & $\begin{array}{l}\text { Bubbles, motion blur, wall } \\
\text { contact }\end{array}$ & 100 & Hard \\
$\mathbf{3}$ & $\begin{array}{l}\text { Out of focus, motion blur, } \\
\text { wall contact }\end{array}$ & 174 & (Medium) Hard \\
$\mathbf{4}$ & Motion blur & 31 & Medium (Hard) \\
$\mathbf{5}$ & Colitis (red, no folds in the & 175 & Medium \\
& wall) & &
\end{tabular}

Table 6.1: Properties and categorization of the test set of image sequences.

With the two best features, the maximum discrimination was 6.1. Yet, the error rate then still was $1.5 \%$ due to not fully normally distributed data. To minimize the error rate all three features were kept and the classifier was implemented in the navigation algorithm.

Figure 6.5 displays plots of target location in $X$ and $Y$ coordinates for a good result with many target location changes (A) and the poorest result (B) in terms of accuracy. In Table 6.2, accuracy and robustness results are summarized. In the top graphs of Figure 6.5 can be seen that automatic target detection follows manually annotated locations really well. The dashed vertical lines indicate areas for which $\Lambda \mathbf{z}$ (zRes, bottom graphs) was far below the threshold, shown for reference as a horizontal line. In the top graphs it is seen that for these regions, the automatic detection corresponds poorly to the manual gold standard.

\begin{tabular}{c||ccl} 
Sequence & ICC in \% & No. of frames & $\begin{array}{l}\text { No. of useful } \\
\text { frames }(\% \text { of total) }\end{array}$ \\
\hline $\mathbf{1}$ & 98.8 & 77 & $64(83.12)$ \\
$\mathbf{2}$ & 89.4 & 100 & $72(72)$ \\
$\mathbf{3}$ & 97.1 & 174 & $131(75.29)$ \\
$\mathbf{4}$ & 95.4 & 31 & $25(80.65)$ \\
$\mathbf{5}$ & 84.7 & 175 & $6(3.43)$
\end{tabular}

Table 6.2: Results of robustness and accuracy of the target detection.

From the results in Table 6.2 can be seen that the ICC between the results of our algorithm and the manual results is high with an average of $93 \%$ (range $84.7 \%$ - 98.8\%). A high overall ICC corresponds to a high number of useful frames according to the classifier. The best result indeed was obtained with sequence 1 , which we categorized as 'Easy'. The poorest result came from sequence 5, which we categorized as 'Medium'.

Sequence 5 only contained $6(3.43 \%)$ useful frames, leading to the question whether the classifier worked properly in this sequence. In Figure 6.6, a representative image 

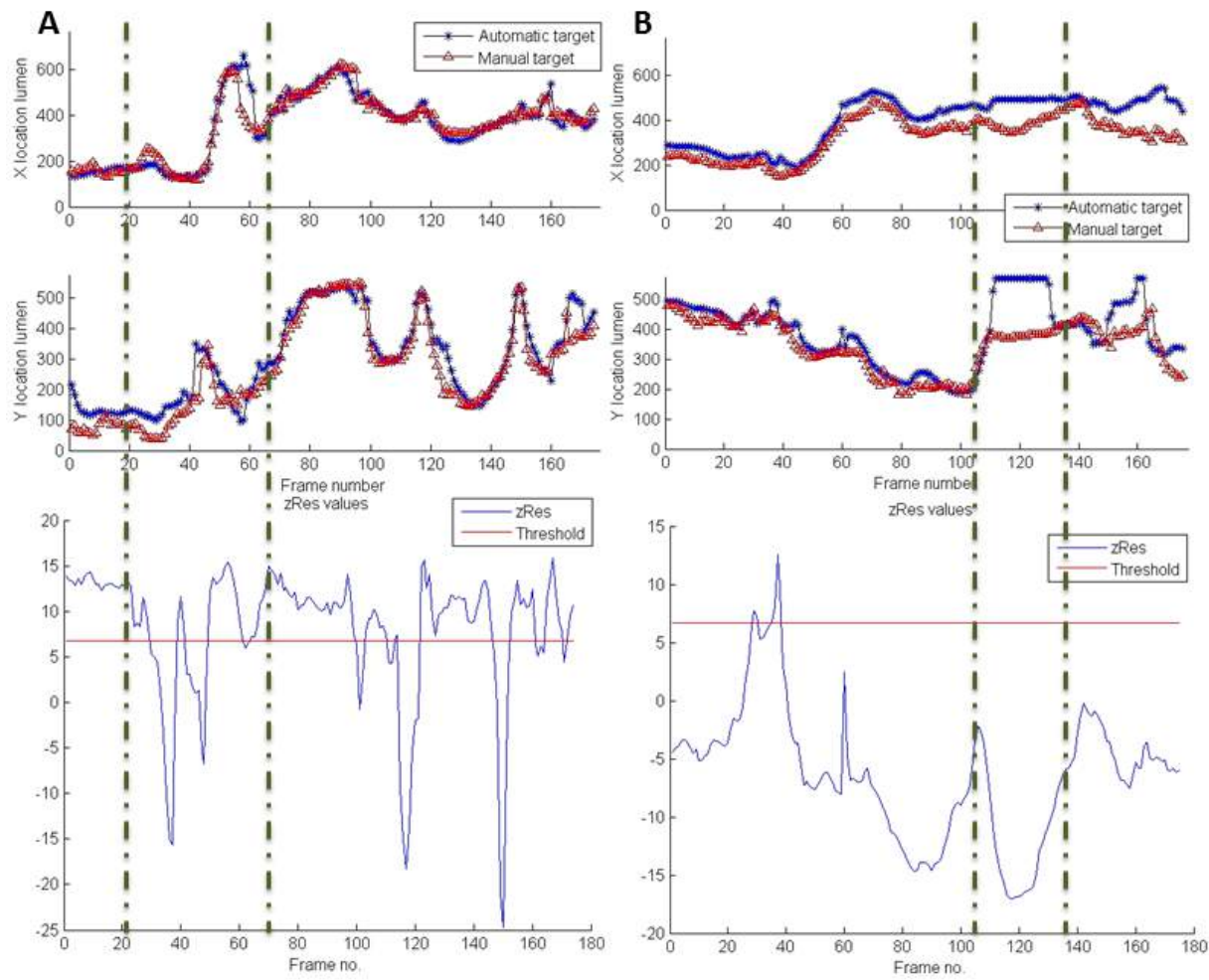

Figure 6.5: The top graphs illustrate the target location results in $\mathrm{x}$ (top) and $\mathrm{y}$ (bottom) coordinates in pixels. The bottom graphs display the log likelihood of $z$, resulting from the classifier. Plots A correspond to sequence 3 , plots B to sequence 5 . The dashed vertical lines show areas where automatic and manual target locations do not correspond very well. In the bottom graphs, low zRes values can be seen in these areas.

from this sequence is displayed. The colon of this patient was inflamed, causing a red appearance without the usual characteristic folds in the wall. The variance and entropy of these images thus deviate from images of patients without this disease, which is why the classifier ruled so many images as 'uninformative'. On the one hand, these frames do contain relevant information and should thus not be classified as such. However, from the results of the target localization algorithm can be concluded that a reliable target is difficult to find in images from this sequence. From a navigation standpoint, the classification is therefore still correct. 


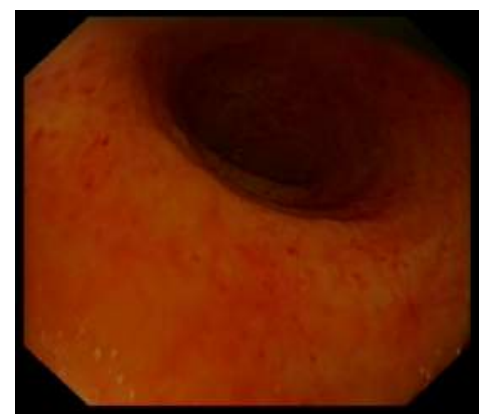

Figure 6.6: Representative image from image sequence 5. The characteristic folds in the wall are missing and the colon wall is unusually vascularized and red. These features influence the classifier result heavily.

\subsection{Conclusion}

In terms of ergonomics and procedure efficiency, clinical relevance of robotized flexible endoscopy is beyond question. Once a robotized system is being considered, imagebased navigation is a logical addition. In this research, an assistive algorithm for endoscope insertion was developed, implemented and evaluated for accuracy and robustness in real human colonoscopy images.

The algorithm was based on dark region segmentation after image classification. Earlier research has been done in the field of dark region segmentation in endoscopy. Chettaoui et al. [30] developed an algorithm that distinguishes the normal lumen from so-called 'diverticulas', bulges in the colonic wall that can be mistaken for lumen. Because the algorithm we developed takes the mean of the $5 \%$ darkest regions, and furthermore because the location is updated 25 times per second, we do not expect significant influence in control performance in case of diverticulas. Moreover, our algorithm is meant as an assistive navigation tool, which means the endoscopist can turn it off at any moment.

Zhiyun describes an extensive analysis on lumen boundary extraction [34]. In section 6.2 was stated that the main requirement of an endoscopic navigation algorithm is real-time, intuitive functionality. The method proposed by Zhiyun is complicated and computationally intensive, making it not very applicable to clinical practice.

In the experiments it was found that the developed target centralization algorithm always detected a suitable target or lumen direction, with an ICC with manual annotation of the target of $93 \%$. The categorization we established beforehand was not predictive for accuracy of the results, but the image classifier that was developed was an excellent predictor of images in which a lumen could be detected reliably. In the sequence from a patient with an inflamed colon, the classifier ruled many frames as 'uninformative', making the algorithm less accurate. However, an ICC of $84.7 \%$ was still obtained in this case. It is therefore expected that this kind of patients can still 
be treated using the described system. The algorithm further works in real-time on standard equipment, making it suitable for current clinical practice.

Although these results are promising, future work will be needed to improve navigation further. Possible improvements are to incorporate heading direction information [45]. Furthermore, user feedback on the system's functionality during experiments in a realistic test environment will be obtained to adapt to clinical practice and increase possible acceptance in the clinic. Finally, clinical relevance of the complete robotized endoscopy system needs to be established. Therefore, the first patient study has started and our goal is to include 32 patients.

\subsection{References}

Please refer to the bibliography at the end of this thesis. 


\title{
Automated lumen centralization for colonoscopy: user evaluation
}

\author{
'There are three principal means of \\ acquiring knowledge... observation, \\ reflection, and experimentation. \\ Observation collects facts; \\ reflection combines them; \\ experimentation verifies the result \\ of that combination.'
}

Denis Diderot

Published as: Hendrikus J.M. Pullens, Nanda van der Stap, Esther D. Rozeboom, Matthijs P. Schwartz, Ferdi van der Heijden, Martijn G.H. van Oijen, Peter D. Siersema, Ivo A.M.J. Broeders. 'Colonoscopy with robotic steering and automated lumen centralization: a feasibility study in a colon model'. DOI 10.1055/s-0034-1392550. ISSN 0013-726X. Endoscopy. Published online: 2015.

Reprinted with permission.

This chapter contains the clinical validation of expert and novice experiments with the robotic flexible endoscopy setup to which Automated Lumen Centralization (ALC) was added. Experiments were performed in an anatomic model. 


\subsection{Introduction}

With the initiation of population screening for colorectal cancer, there has been a steady increase in the demand for colonoscopy in many countries [109, 14]. Physicians entering colonoscopy training programs face extensive learning curves and must perform several hundred colonoscopic procedures before being able to intubate the cecum in the vast majority of patients $[110,111]$. This difficulty can at least partly be attributed to the nonintuitive steering mechanism of flexible endoscopes. Furthermore, musculoskeletal complaints due to the non-ergonomic design of the colonoscopy setup have been reported to occur in up to $50 \%$ of endoscopists [112]. Colonoscopes with a more intuitive and ergonomic steering mechanism might reduce learning curves and improve the efficiency of colonoscopic interventions, subsequently increasing colonoscopic capacity.

Robotics have the potential to overcome the challenges encountered in endoscope control. Technical studies on the use of robotic steering in gastrointestinal endoscopy have, however, yielded diverse results [99, 113, 33]. During both introduction and withdrawal of the colonoscope, it is important that the tip of the colonoscope be oriented in a manner that optimizes overview of the colonic anatomy. Robotic control of flexible endoscopes allows automated visual flexible endoscope navigation. This can help to keep the tip of the scope oriented in the colonic lumen.

We introduced a robotic platform with the option of automated lumen centralization (ALC) to assist the endoscopist in steering the endoscope tip and performed a randomized, crossover pilot study with a colonmodel to evaluate the feasibility of colonoscopy with robotic steering and ALC (RS-ALC).

\subsection{Participants and methods}

\subsubsection{Study population}

Both expert endoscopists and novices participated in the study. The expert endoscopists were gastroenterologists from two hospitals (Meander Medical Center, Amersfoort, the Netherlands, and University Medical Center, Utrecht, the Netherlands), all with individual experience of more than 2000 colonoscopies. The novices were students of technical medicine at the University of Twente, Enschede, the Netherlands. All had basic knowledge of gastrointestinal anatomy and pathophysiology, and all knew the technical principles of gastrointestinal endoscopy. None of the novices had any experience in performing endoscopy.

\subsubsection{Study design}

All participants performed colonoscopies on a physical colon model with both conventional steering and RS-ALC. We used a crossover design in which participants were randomized to which of the two modalities they were to use first. Before testing, each participant received both verbal and written instructions on the goals of the study, the colon model, and RS-ALC. Novices also received instructions on conventional steering. 
Expert endoscopists had 5 minutes in which to become familiar with the colon model while using the conventional steering method and 20 minutes to practice with the robotic setup before the study started. Noviceswere granted 10 minutes to get used to the colon model and 20 minutes to practice each modality. Participants were instructed to have the endoscope reach the cecum as fast as possible. Withdrawal time was set at 6 minutes. During testing, we allowed no additional instructions. Participants who were not able to reach the cecum with one of the steering modalities were excluded.

After completion of the tests, the participants filled out a questionnaire with dichotomous questions on their subjective evaluation of the new platform.

\subsubsection{Colon model with simulated polyps}

All procedures were performed with an Olympus Exera II CLV-180 endoscopy platform and colonoscope (Olympus, Tokyo, Japan). We used the Kyoto Kagaku Colonoscope Training Model (Kyoto Kagaku Co. Ltd, Kyoto, Japan), which is a physical colon model consisting of a life-size plastic torso with a synthetic colon inside (Figure 7.5). The colon is threaded through rubber rings that are attached to the torso, either directly or with springs. The colon was configured into standard cases, according to the layout guides provided by the manufacturer.

Expert endoscopists used case 2 of the colon model to perform the tests, which is one of the cases that has previously been validated for assessing colonoscope insertion skills [114]. During pretesting of the platform setup for feasibility, none of the novices was able to reach the cecum with either modality while using this case. Therefore, novices performed the tests on case 1 , which is easier.

We manually applied 21 foam fabric simulated polyps, varying in size, throughout the colon in a distribution similar to that reported by Gralnek et al. [115]. The novices used a shorter part of the synthetic colon for case 1, so the simulated polyps were redistributed to obtain the same distribution per colonic segment as in case 2 (Figure 7.1). The participants were blinded with regard to the number of simulated polyps and the fact that they performed both procedures on the same case of the colon model.

\subsubsection{Robotic steering with automated lumen centralization}

When RS-ALC was used, the angulation wheels of the endoscope were connected to a remote drive unit and placed in a docking station (Figure 7.6) [53]. The user steered the tip by means of a joystick controller (Figure 7.2). To compensate for the lack of tactile feedback from the angulation wheels, a feedback circle was shown on screen to indicate to the participant in which direction and to what extent the tip of the colonoscope was bent [116].

ALC consisted of a software algorithm that identifies the darkest pixels in the image [77]. The darkest region in the image usually corresponds to the middle of the colonic lumen, which is the target area for the colonoscope. On screen, a small circle continuously depicted the target as detected by the ALC algorithm. When the position of the circle corresponded to the actual target, the participant could actively decide to let the platform steer the scope to center this point in the endoscopic image. This was 


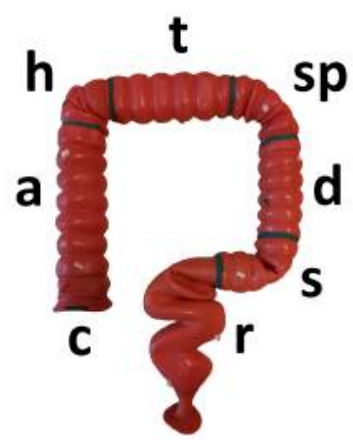

case 1

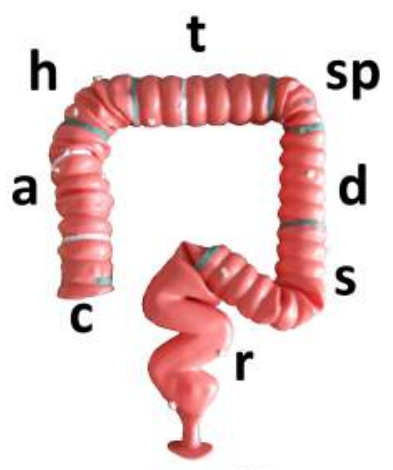

case 2

Figure 7.1: The cases of the colon model with distribution of the simulated polyps: c, cecum; a, ascending colon (3 simulated polyps); h, hepatic flexure (3 polyps); t, transverse colon (2 polyps); sp, splenic flexure (3 polyps); d, descending colon (2 polyps); s, sigmoid colon (4 polyps); r, rectum (4 polyps).

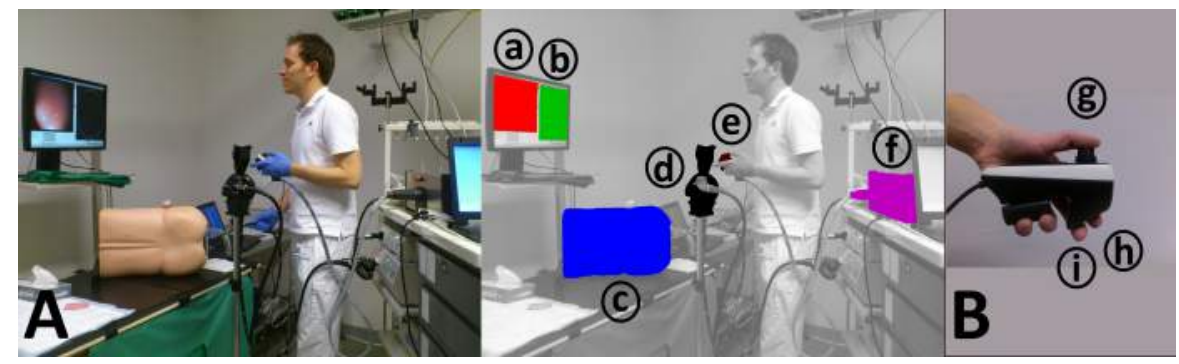

Figure 7.2: a Setup of the experiment: a, endoscopic image; b, visual feedback circle; c, Kyoto Kagaku Colonoscope Training Model; $d$, docking station; e, joystick controller; $f$, motor unit. $b$ Details of the joystick controller: g, joystick; h, button 1; i, button 2 .

done by pressing and holding button 1 on the joystick controller. Releasing the button immediately stopped the platform from steering the tip.

All tests were performed single-handedly, with the joystick controller or conventional colonoscope steering mechanism in one hand and the shaft of the colonoscope in the other. With both modalities, the colonoscope could be torqued as usual.

\subsubsection{Study end points}

Study end points were the cecal intubation time, number of detected simulated polyps during endoscope withdrawal, and participants' subjective evaluation of the new platform. 


\subsubsection{Statistical analysis}

We performed data analysis with SPSS version 22 (IBM, Armonk, New York, USA). For both participant groups, we used Wilcoxon's signed rank test to compare the median cecal intubation times and numbers of detected simulated polyps achieved with the robotic method and the conventional method. We used Fisher's exact test to compare the median "on" times of the two participant groups for the ALC option. Categorical variables were also compared with Fisher's exact test. All tests were two-tailed. We considered a $P$ value of less than 0.05 to be statistically significant. Because this was a pilot study, no power calculation was performed beforehand.

\subsection{Results}

A total of 8 expert endoscopists ( 7 men, median age 47 years [interquartile range (IQR) 42.25-56.25]) and 12 novices ( 3 men, median age 21.5 years [IQR 20-22]) participated in the study. We excluded the data for 2 novices from further analysis, one of whom failed to reach the cecum during colonoscopy with conventional steering (randomized to RSALC first) and one of whom failed to complete the first procedure without additional instructions (randomized to start with conventional steering).

Novices required a shorter time to intubate the cecum with RS-ALC (median 8 minutes [min] 56 seconds [s], IQR 6min 46s-16min 34s) than with conventional steering (median 11 min 47s, IQR 8min $19 \mathrm{~s}-15 \mathrm{~min} 33 \mathrm{~s}, P=0.65$ ) (Figure 7.3). The intubation time of expert endoscopists was shorter with conventional steering (median $2 \mathrm{~min} 9 \mathrm{~s}$, IQR 1min 13s-7min 28s) than with RS-ALC (median 13min 1s, IQR 5min 9s-16min 54s, $P=0.12$ ). The intubation times did not differ between the randomization groups in either the experts or the novices.

Novices detected more polyps with RS-ALC than with conventional steering (median 88.1\% [18.5/21], IQR 79.8\%-95.2\% vs. median 78.6\% [16.5/21], IQR 75.0\%-91.7\%, $P=0.17)$, whereas expert endoscopists found significantly fewer polyps with RS-ALC than with conventional steering (median 69.0\% [14.5/21], IQR 61.0\%-75.0\% vs. median 80.9\% [17.0/21], IQR 76.2\%-85.7\%, $P=0.03$ ) (Figure 7.4).

During endoscopy with robotic steering, we found no significant difference in regard to the median percentage of overall time during colonoscopy that the ALC option was switched on between the expert endoscopists (7.3\%, IQR 3.3\%-13.6\%) and the novices (2.6\%, IQR 2.4\%-4.1\%) $(P=0.153)$.

Table 7.1 shows the results of the post-procedural interviews regarding RS-ALC. All novices and four expertswere generally positive about RS-ALC. All participants but one agreed that RS-ALC makes performing colonoscopy easier for novices, but not for experienced endoscopists. All novices thought that RS-ALC made introducing the colonoscope easier and performing colonoscopy overall faster in comparison with conventional steering. 


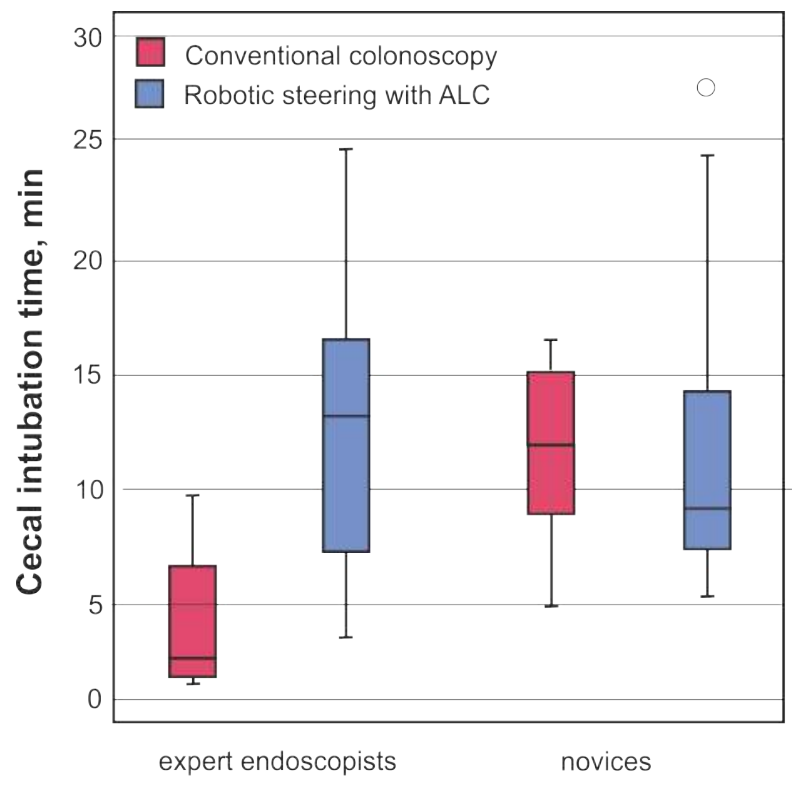

Participants

Figure 7.3: Box and whisker plot with cecal intubation times for expert endoscopists and novices using conventional colonoscopy or robotic steering with automated lumen centralization (ALC).

\subsection{Discussion}

This colon model study shows that the use of RS-ALC is feasible in both expert endoscopists and novices. Post-procedure interviews indicated that RS-ALC was considered most appropriate for novices.

Overall, the proportion of simulated polyps detected by the participants in our study during colonoscopy with conventional steering was larger than the $52.9 \%$ of simulated polyps detected by the expert endoscopists in the in vitro colonoscopy study by Gralnek et al. [115]. We based the location of the simulated polyps in our colon model on the model used by Gralnek et al., but instead of metallic beads, we used simulated polyps made of foam fabric. These may have been easier to identify.

Allemann et al. and Zhang et al. previously evaluated the performance of a motorized conventional endoscope with a joystick interface $[99,113]$. Their results were somewhat disappointing, possibly owing to the fixed position of the endoscope in their experimental setup, which limited maneuverability and proprioceptive feedback. Reilink et al. reported no significant difference between cecal intubation time with conventional steering and intubation time with an intuitive interface when novices were allowed to perform simulated colonoscopy [33]. The design of our platform is different from that of previous studies as it still allowed manual handling of the shaft of the 


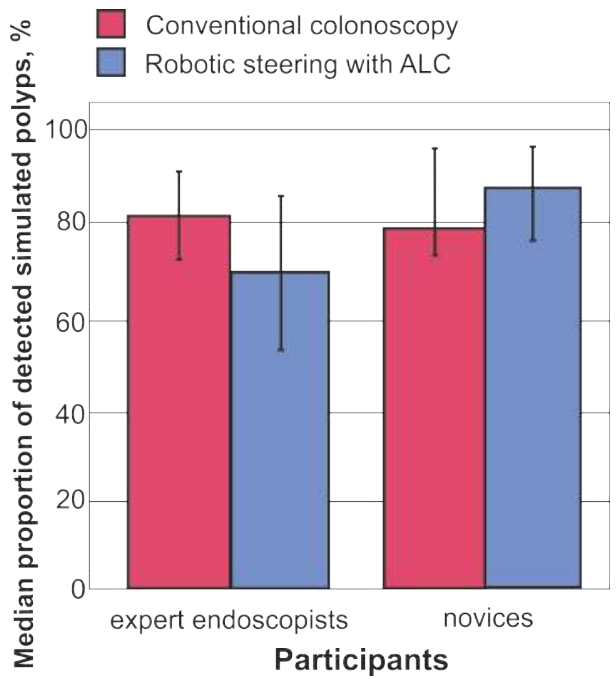

Figure 7.4: Bar graph with median polyp detection rates for expert endoscopists and novices using conventional colonoscopy or robotic steering with automated lumen centralization (ALC). A rate of $100 \%$ equals detection of 21 simulated polyps. Error bars indicate $95 \%$ confidence interval.

endoscope. Therefore, our platform is more comparable with normal clinical practice.

The strengths of this study are the randomized, crossover design, which prevented the influence of a learning effect with the colon model. The novices in our study were well matched and comparable with fellows starting training in gastrointestinal endoscopy. Furthermore, we used a colon model that several cases had previously validated [114].

A potential drawback of this study is that the time to practice on the colon modelwas short. Arguably, 20 minutes of practice is too short, especially for noviceswho are completely endoscopy naive. This is probably also reflected in the fact that the participants had the ALC option turned on during only a small proportion of the total colonoscopy time. Participants were asked to combine many different cognitive and motor tasks that were new to them. Pressing and holding an additional button may have been too much to ask. The current experimental setup might therefore not be the optimal way to evaluate the intuitiveness of the ALC option.

Nonetheless, considering that this was a feasibility study and as such not designed and powered to detect significant differences between the different modalities and participant groups, the results are promising. A direct comparison between RS-ALC and conventional steering in the hands of expert endoscopists was not possible because of the fact that they had already had considerable experience in conventional steering, whereas the ALC platform was entirely new to them. All expert endoscopists were, however, able to reach the cecum with RS-ALC. Finally, no conclusions can be drawn regarding the longevity of the finding that RS-ALC appears to be easier and more in- 


\begin{tabular}{|c|c|c|c|}
\hline Statement & $\begin{array}{l}\text { Agrees with state } \\
\text { Expert endosco- } \\
\text { pists }(n=8), n(\%)\end{array}$ & $\begin{array}{l}\text { Novices } \\
(n=10), n(\%)\end{array}$ & $P$ value* \\
\hline \multicolumn{4}{|l|}{ Colonoscopy with RS-ALC... } \\
\hline $\begin{array}{l}\text {...makes introduction of the } \\
\text { scope easier. }\end{array}$ & $3(37.5)$ & $10(100.0)$ & 0.007 \\
\hline $\begin{array}{l}\text {...makes performing endo- } \\
\text { scopy faster. }\end{array}$ & $2(25.0)$ & $10(100.0)$ & 0.002 \\
\hline $\begin{array}{l}\text {...is more intuitive than con- } \\
\text { ventional colonoscopy. }\end{array}$ & $4(50.0)$ & $9(90.0)$ & 0.118 \\
\hline $\begin{array}{l}\text {...makes performing en- } \\
\text { doscopy easier for novices. }\end{array}$ & $7(87.5)$ & $10(100.0)$ & 0.444 \\
\hline $\begin{array}{l}\text {...makes performing en- } \\
\text { doscopy easier for experts. }\end{array}$ & $1(12.5)$ & $1(10.0)$ & 1.00 \\
\hline $\begin{array}{l}\text { I am positive about this plat- } \\
\text { form. }\end{array}$ & $4(50.0)$ & $10(100.0)$ & 0.023 \\
\hline $\begin{array}{l}\text { I see a potential role for this } \\
\text { platform in clinical use. }\end{array}$ & $5(62.5)$ & $8(80.0)$ & 0.608 \\
\hline
\end{tabular}

Table 7.1: Subjective evaluation of experience with and future use of robotic steering with automated lumen centralization (RS-ALC).

tuitive for endoscopy-naive novices because each participant performed only one examination with each modality.

Our study shows the potential use of RS-ALC, especially in inexperienced endoscopists. The possible additional value of the current platform would best be indicated by the learning curve of endoscopy-naive novices. For endoscopists in training, this platform might be attractive because it can be used as a click-on system with existing and readily available endoscopy equipment. Before the platform is introduced into clinical practice, however, further studies are required. The next step could be a randomized trial evaluating the learning curves of fellows in gastroenterology using either the conventional steering mechanism or RS-ALC at the start of training. The primary end point in such a study should be the cecal intubation time.

In conclusion, performing colonoscopywith RS-ALC is technically feasible. Its main advantages appear to be its intuitiveness for inexperienced endoscopists, whose evaluation in our study regarding the currently presented platform was unequivocally positive.

\subsection{Acknowledgements}

The authors would like to thank all the expert and novice endoscopists for their participation in this study. 


\subsection{References}

Please refer to the bibliography at the end of this thesis.

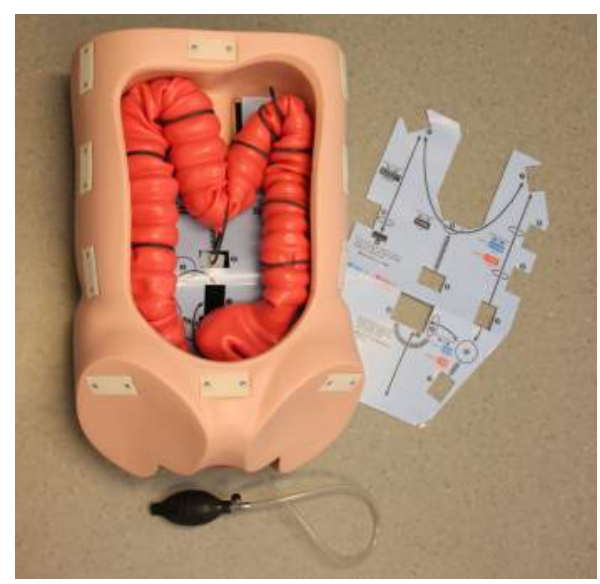

Figure 7.5: The Kyoto Kagaku Colonoscope Training Model with an example of a layout guide for standard cases, as provided by the manufacturer.

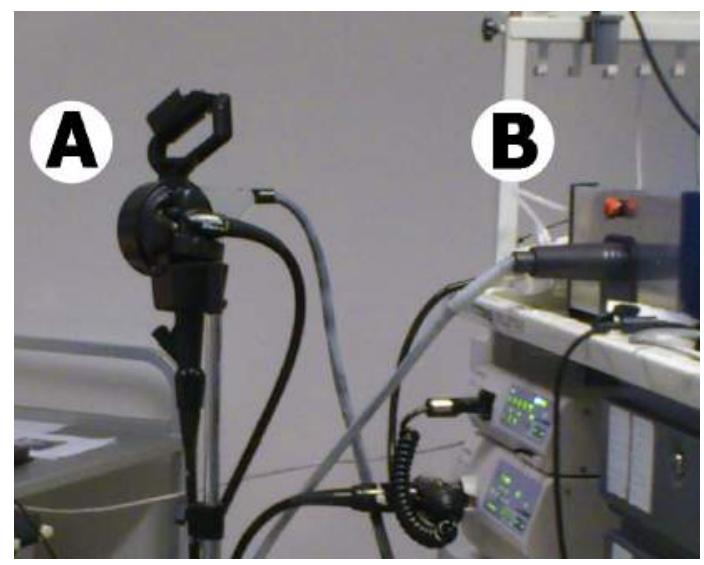

Figure 7.6: Close-up view of the steering mechanism of the robotic steering with automated lumen centralization (RS-ALC) platform. The angulation wheels of the colonoscope are placed in a docking station and connected with the mobile drive unit, which is connected to the motor unit and the joystick controller. The steering wheels of the colonoscope can easily be placed in the docking station by means of a click-on system. A, mobile drive unit; B, motor unit. 
CHAPTER 8

\section{Technical Validation of Automated Lumen Centralization}

Ronald Reagan

Published as: N. van der Stap, E.D. Rozeboom, H.J.M. Pullens, F. van der Heijden, I.A.M.J. Broeders. 'Feasibility of Automated Target Centralization in Colonoscopy'. International Journal of Computer-Assisted Radiology and Surgery (IJCARS, ISSN 1861-6429), 8 October 2015 (online). DOI: 10.1007/s11548-015-1301-3

This chapter contains the technological validation of Automated Lumen Centralization (ALC), implemented in the robotic flexible endoscopy setup 'Teleflex'. Experiments were performed in an anatomic model. 


\subsection{Introduction}

Colorectal cancer has one of the highest incidences of all cancers in the Western world [117]. Colonoscopy, inspection of the colon with an endoscope (Figure 2.1), is a vital tool in the screening procedure for colorectal cancer. It is a useful next step after a positive Fecal Occult Blood Test (FOBT), which is the first step in national screening programs in many European countries [118]. In the Netherlands a national population screening program was started in 2014. This program is expected to increase the number of colonoscopies by 70.000 yearly [23]. Currently, approximately 190.000 colonoscopies are performed each year, which implies an increase in demand of over $35 \%[119,13]$. Controlling the endoscope is difficult to learn; starting endoscopists require a learning curve of 100-300 procedures to reach competency in colonoscopy [18]. Colonoscopes are steered using two large steering knobs (Figure 2.1) that steer the tip using Bowden cables. Only the tip of the colonoscope $(+/-8 \mathrm{~cm})$ can thus be controlled actively, the flexible shaft of the endoscope follows passively [19]. This 60-year old non-ergonomic control section also causes physical complaints [120], a consequence that reduces the colonoscopic capacity while demand rises. With our research, we aim to improve intuitiveness and ergonomics of the endoscope. We are focusing on colonoscopy because of the clear demand.

A typical colonoscopy procedure consists of a retrograde insertion phase and a retraction phase. The insertion is done as quickly as possible until the beginning of the organ, the cecum, is reached. Retraction has a recommended duration of at least six minutes [121]. During this phase, the colon wall is inspected for anomalies which are removed if necessary $[19,122]$. Commonly found anomalies are so-called 'polyps', uncontrolled growth of the mucosa on the colon wall. Due to a minimal retraction time, procedure efficiency and colonoscopy capacity can only be improved by shortening the insertion phase. An easier control mechanism is expected to make endoscope insertion faster and the learning curve shorter.

Robotic systems with intuitive controllers such as a remote joystick have been shown to reduce the experienced workload and improve control intuitiveness for endoscopists [116]. Image-based navigation may help to improve intuitiveness of robotic systems even further [83]. During the insertion phase, image-based navigation could be useful in finding the target direction and steering towards it automatically. The colon is visible with a colonoscope as a tubular, folded structure. The target of the colonoscope almost always is the deepest visible area, often corresponding to the center of the lumen. This area usually presents as the darkest area in the endoscopic images, which is a useful feature for image-based navigation.

Central lumen detection for automatic endoscope steering has been investigated before [36, 38, 34, 123, 28]. Automated endoscope steering was reviewed as well [83]. Most of the research in this area focuses on segmenting the central lumen area as accurately as possible. Although accurate central lumen detection in colonoscopic images is technologically feasible, none of the mentioned systems to our knowledge are clinically accepted or even tested for clinical applicability.

All previous techniques are based on the assumption that by centralizing the lumen, the colonoscope will travel the right path through the colon. Complicating factors 
herein are image artifacts, such as fluids or bubbles on the lens, which make images hard to interpret. The lens may also be pressed against the colon wall, causing a 'redout' or 'wall view'. Additionally, the camera can be moved substantially between frames, causing motion blur artifacts [36]. These complicating factors and artifacts have impeded successful implementation of this technique up to now. Moreover, centralizing the lumen is not always desired by the endoscopist. Sometimes, maneuvers using the colonic wall are performed on purpose to advance the endoscope further $[124,125]$.

We have developed and evaluated a new algorithm for colonoscopy steering based on dark region centralization [20]. This algorithm is implemented in an assisting fashion, and predicts whether images will contain useful information. The prediction diminishes the influence of artifacts. This algorithm was adapted to be implemented in a robotized flexible endoscopy system called Teleflex $[53,126]$. The vision-based functionality is meant to assist during procedures, and can be actively turned off and on by the endoscopist.

The aim of the current study is to evaluate the assisting automated lumen centralization algorithm in terms of technical feasibility in a clinical setting. Clinical feasibility means that the system enables colonoscopy that is at least as efficient and effective as the conventional method (non-inferiority), but this was reported on elsewhere [102]. Technical feasibility is defined as the system's performance during colonoscopy and includes user feedback on system functionality. However, the emphasis of this study is on the technical performance of the robotic system as a whole.

\subsection{Materials}

Experts in endoscopy and inexperienced participants performed colonoscopy on a simulated colon model using either the conventional endoscopic steering method (turning the steering knobs), or the robotized setup (Figure 8.1).

\subsubsection{Robotic setup with target centralization algorithm}

There were three main requirements for control of the complete system. First, real-time functionality was essential. The procedure needed to be executable without the endoscopist having to wait for visual feedback from the system. Second, the endoscopist needed to be able to overrule the algorithm instantaneously at any point. Third, the complete functionality of the system needed to be intuitive, which means it should be easily learned and implemented in clinical practice. In our system, control of the tip in the robotized setup could either be established through remote user input (e.g. a joystick device), or through the image-based navigation algorithm.

An algorithm was developed with these requirements in mind and implemented in a robotized endoscopy system [53]. In the robotized endoscope setup the tip of the endoscope is controlled through telemanipulation using a joystick controller (Figure 8.2). This interface has been validated before $[116,127,128]$. If a designated button was pressed and held on the joystick controller (arrow in Figure 8.2), steer commands were generated by the navigation algorithm. The user thus had to actively choose if the steering would be controlled by the algorithm. For both types of input commands 


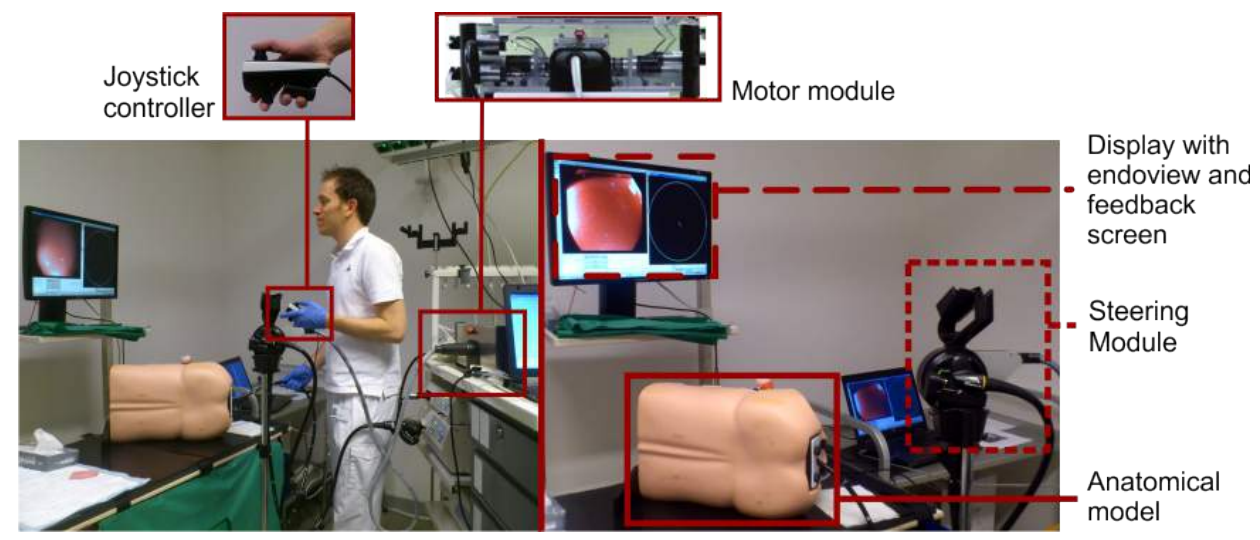

Figure 8.1: The robotized experimental setup for this study. The endoscopist looks at the display and controls the endoscope with the joystick to perform a colonoscopy on the anatomical model. The robotic parts were removed during conventional steering.

the same control loop was passed in real-time (Figure 8.3).

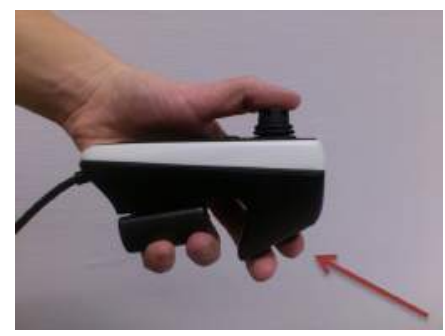

Figure 8.2: The button to enable automated navigation could be pressed by the forefinger.

The robotic setup consisted of a conventional flexible endoscope with the handle linked to the steering module $[53,127]$. The handle and the steering module were suspended on a custom-designed holder. The tip of the endoscope could then be manipulated through motor control, enabled by the joystick controller and a standard laptop, while shaft insertion and rotation were done manually.

The assisting navigation algorithm's main task was to detect the target of the endoscope, which was the lumen center, through image analysis. It used information from previous images to estimate the target location and corrected with current image information (Figure 8.4). Let $f_{i}(\mathbf{y})$ be an image sequence with frame index $i$ and pixel positions $\mathbf{y}$. The estimate of the pixel position of the lumen center $\mathbf{x}(i)$ of frame $i$ will be called $\mathbf{x}(i) . \mathbf{x}(i)$ is obtained following the CoG computation in [32]. All other frames are processed as displayed in the flowchart (Figure 8.4). To suppress noise influence, Gaussian low-pass filtering is applied, and subsequently the image is inverted. Then, 


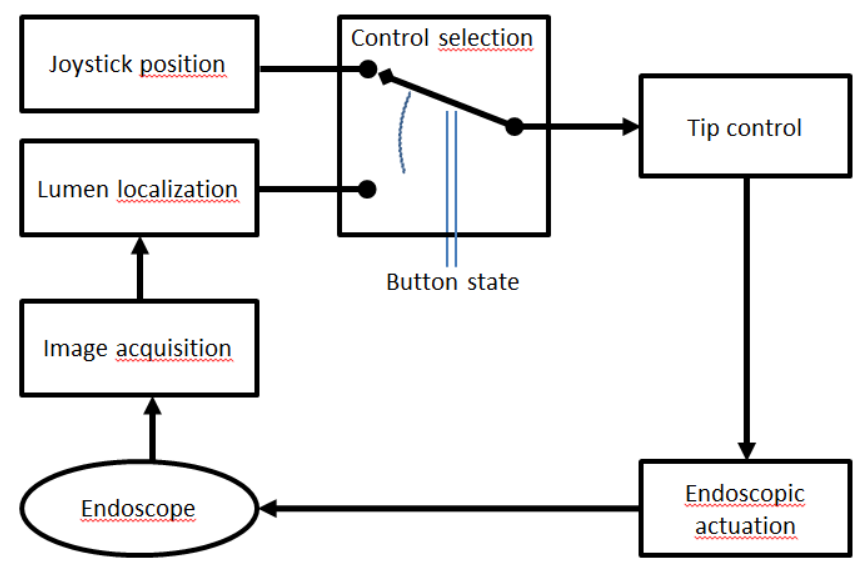

Figure 8.3: Control loop of the robotic flexible endoscope system that was used in this study.

a maximum needs to be found instead of a minimum. The Gaussian convolution ensures windowing the maximum towards the previously estimated target position. Iteration was applied to increase the bias the Gaussian convolution causes, meaning larger shifts of the target between frames still resulted in accurate target estimation. Performance of the algorithm was evaluated in a previous study using human colonoscopy images [77].

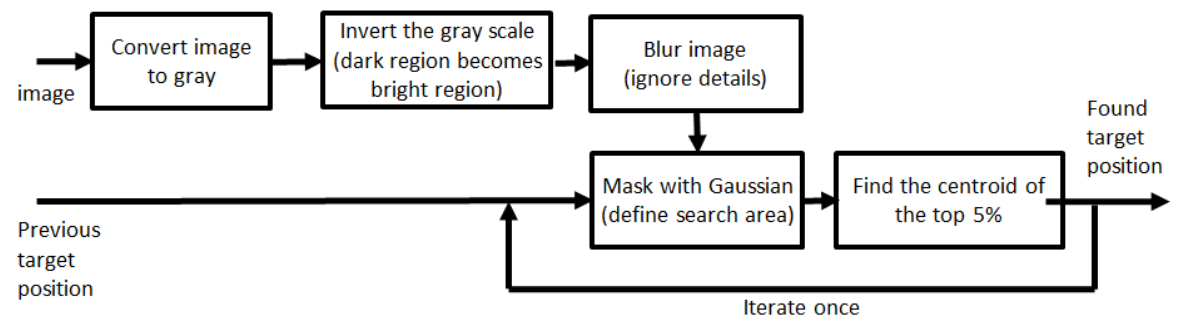

Figure 8.4: Flowchart of algorithm steps. The current image and the previous target are used as input to find the current target location.

Participants were asked to perform a procedure on a plastic, earlier validated, anatomical model (Kyoto Kagaku, Kyoto, Japan) [114]. For each configuration, 21 foam 'polyps' were applied on the inside of the colon. The polyps corresponded in size and location to the polyps described in [115]. 


\subsection{Methods}

Eight expert endoscopists (each performed $>1000$ endoscopic procedures) and ten inexperienced technical medicine students (without experience in endoscopy but with knowledge of anatomy, physiology and pathology of the colon and abdomen (novices)) performed a simulated colonoscopy on the plastic model of the colon. The participants were asked to perform a colonoscopy twice: once using the conventional steering knobs, once using the robotic setup with the assisting target centralization function (Automated Target Centralization, ATC). The order in which they performed the procedures was randomized: half of the participants started with the robotic method, and half of the participants with the conventional method. They were asked to intubate the endoscope as fast as possible, and retract in six minutes while inspecting the bowel wall for lesions. Afterward, participants were asked for their subjective opinion by means of a questionnaire.

Novices performed a colonoscopy in the simplest colon configuration (case 1, Figure 8.5). Experts performed the experiments on a more complicated configuration because of their experience with the colonoscopy procedure itself (case 2, validated in [114]). This distinction was made after pretesting both groups. Novices were not able to complete case 2 with any of the two modalities, while experts were unrealistically fast in completing case 1 . This did not hinder study evaluation because the performance was not compared between groups, but between modalities. The polyps inside the model were to be detected upon retraction of the endoscope to determine the competence in anomaly detection.

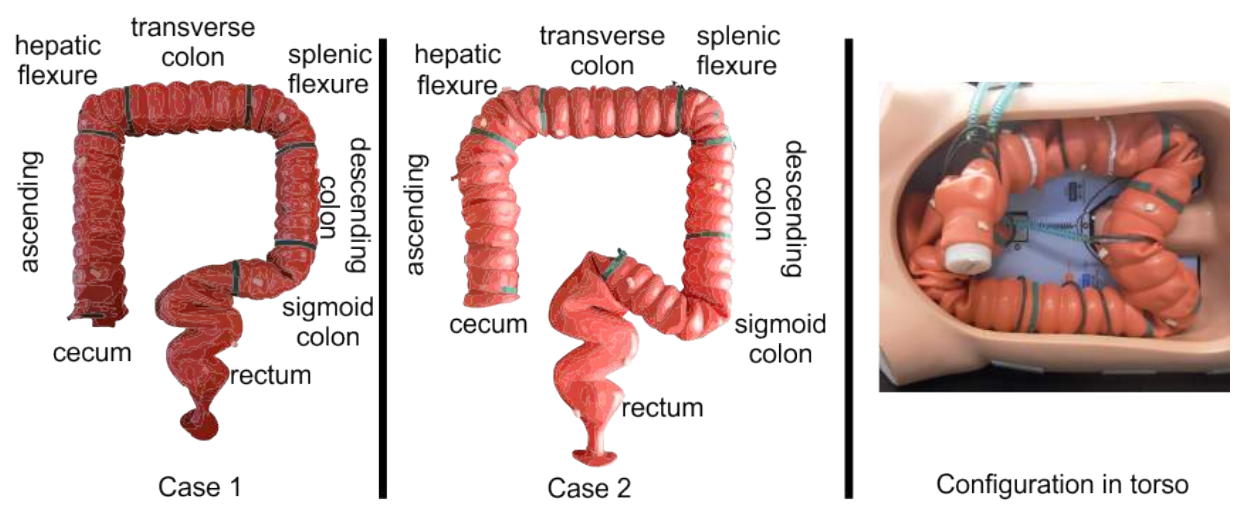

Figure 8.5: Case configurations 1 (left, novices) and 2 (middle, experts). Anatomical names for colon segments are also illustrated per case. Segments are bordered by the green lines to ensure logging consistency between experiments. On the right, the configuration inside the torso during the experiments is shown. This configuration was invisible to participants.

Participants continuously received visual feedback of the endoscopic image and feedback of the tip bending state when using the robotic setup, as depicted in Figure 
8.6. In the left pane (A), a small white circle depicts the target found by the algorithm in the endoscopic image. When using the robotic setup, the participant could choose to press a button and activate ATC if the position of this circle corresponded to the desired steering direction. This circle was always visible, even during the conventional colonoscopic procedures. The right pane (B) shows the amount and direction of tip bending, currently illustrating a tip that is bent halfway downward.

All participants used the same Olympus CF180 colonoscope connected to an Olympus CV-180 Evis Exera II video processor. This type of colonoscope produces $576 \times 768$ pixel images at 25 frames per second. It has a field of view of $170^{\circ}$ and a field depth of 3 to $100 \mathrm{~mm}$. The ATC algorithm was implemented in Python 2.7 [129] using OpenCV [130] on a standard Windows laptop (Dell Probook 6560b). Analyses were done using IBM SPSS Statistics 20 (IBM, Armonk, NY, USA) and Matlab R2011b (The Mathworks Inc., Natick, MA, USA).

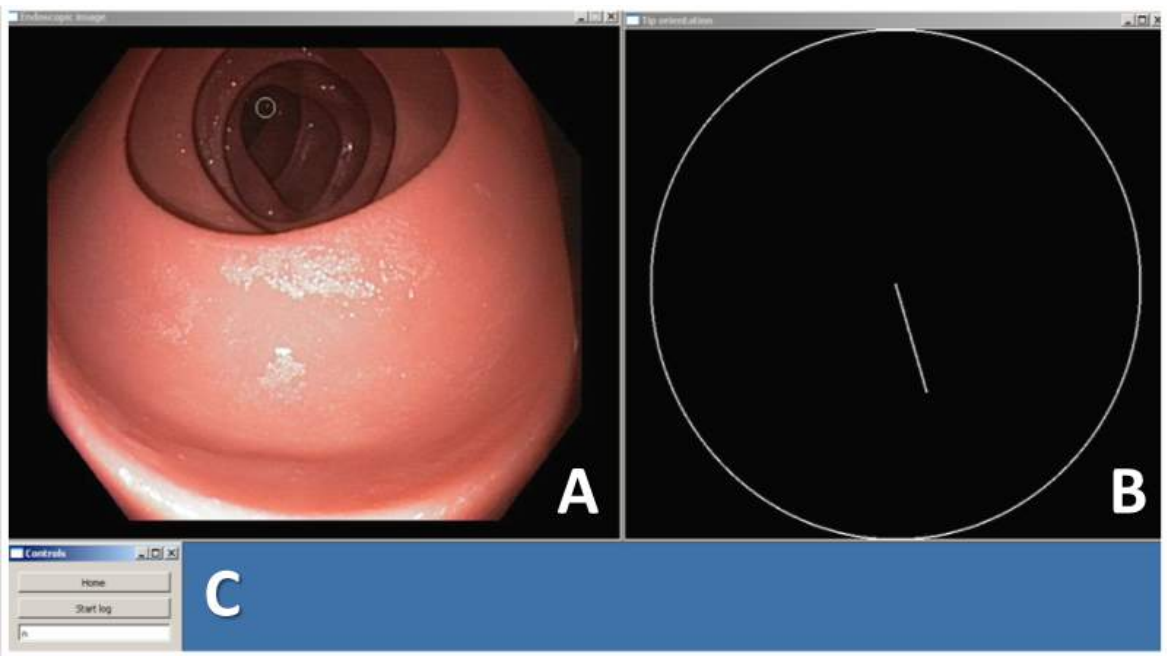

Figure 8.6: Screenshot of a representative situation during the procedure. In the endoview (A) a small white circle indicates the target position in the image. The tip bending diagram shows the current amount and direction of tip bending (B). A small window, needed for logging and changing settings during the procedure, was continuously present but could be ignored by the test participants (C).

\subsubsection{Evaluation parameters and statistical analysis}

Main evaluation parameters were: the percentage of time using ATC (ATC use, \% of total), the time ATC was on (TO, number of times), the 'on'-time per period (DUR, in frames) and subjective user feedback for technical feasibility.

Technical feasibility focuses on the use of the system and can be compared between the two participant groups. The colon was divided into seven segments (Figure 
8.5) and transition of the endoscope from one segment to the other was timed. This was done to enable parameter evaluation per segment. The earlier clinical evaluation was done to compare the different colonoscopy methods, and therefore the comparison was made within the two participant groups.

It was expected that the ATC would predominantly be used in the longer, straight segments of the colon. In these areas the view on the lumen is optimal which would mean the algorithm and the user should agree. We also hypothesized that if ATC would be turned 'on', the introduction would be faster than without centralization.

Overall statistical evaluation was done, if relevant, using a Mann-Whitney U-test with a significance level $p$ of 0.05 . In some cases, the Mann-Whitney U-test could not be used since there was no variance homogeneity. Those cases are clearly indicated in the Results Section, together with the test that was used and the significance level.

\subsection{Results}

The technical feasibility results of the system are described below. For completeness, a summary of the clinical results is provided as well.

\subsubsection{Technical feasibility: use of the system}

ATC was turned on by the novices for a median of $4.2 \%$ of the time during insertion and $0.3 \%$ during retraction. Experts turned ATC on for $4.0 \%$ of the time during insertion and $11.6 \%$ during retraction. The number of times ATC was turned on was 77 times per procedure for the experts and 59 for the novices (Figure 8.7).

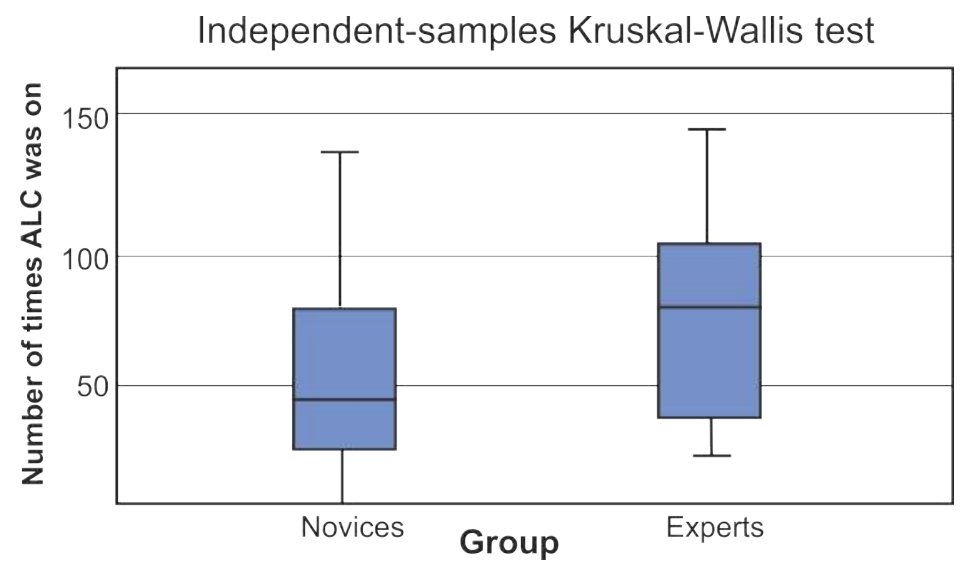

Figure 8.7: Number of times that automatic lumen centralization was turned on ( $y$-axis) per group (x-axis). Note that a Kruskal-Wallis test was used (significance level $p$ of 0.05 ).

The mean duration of ATC use (DUR) was significantly longer in the expert group than in the novices group $(p<0.001)$. Median DUR was 12 frames for experts and 7 frames 
for novices (Figure 8.8).
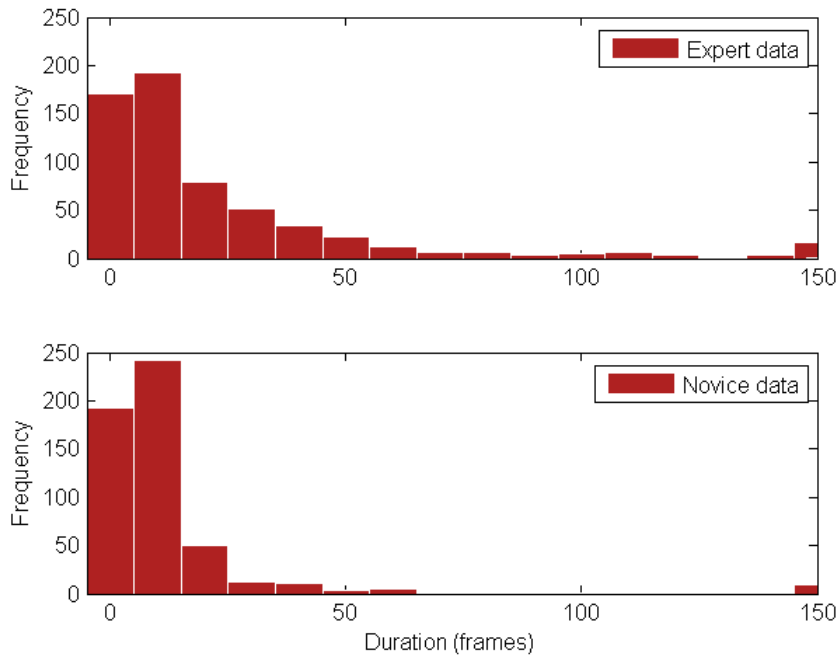

Figure 8.8: Histogram showing mean duration of ATC use (DUR) for experts (top) and novices (bottom).

\section{Subjective questionnaire results}

All novices and three experts thought that endoscope insertion was easier with the robotic system with ATC. However, at least four experts indicated that they expect additional value of the ATC functionality during retraction. The easy rotation of the endoscope tip with the joystick, used for colon wall lesion inspection, became even easier when the tip could be centralized automatically, so when ATC was enabled. Almost all users agreed that the robotic system would make performing a colonoscopy easier for novices (10 novices, 7 experts). 50\% of the experts and all novices were positive about the platform. An additional finding (not in the questionnaire) was that during the experiments some test persons indicated that they 'followed the white circle' while inserting the endoscope during the conventional procedures.

\subsubsection{Clinical feasibility: efficiency and effectiveness}

All included participants reached the cecum using both steering methods. Novices showed no significant differences in time to cecum (TTC) between using conventional (median 11 minutes ( $\mathrm{min}) 47$ seconds (s), with Q1 - Q3 8min 19s - 15min 33s) or robotic control (median 8min 56s, Q1-Q3 6min 46s - 16 min 34s, $p=0.65$ ). Experts showed a trend towards a faster introduction using the conventional method (median $2 \mathrm{~min} 9 \mathrm{~s}$, Q1-Q3 1min 13s - 7min 28s) than with the robotic method (median 13min 1s, Q1-Q3 $5 \min 9 \mathrm{~s}-16 \min 54 \mathrm{~s}, p=0.12$ ). 


\begin{tabular}{c||lcl} 
Segment (insertion) & $\begin{array}{l}\text { Median } \\
(\mathbf{s})\end{array}$ & $\begin{array}{l}\text { robotic } \\
\text { Median } \begin{array}{c}\text { con- } \\
\text { ventional (s) }\end{array}\end{array}$ & $\begin{array}{l}\text { Significance } \\
\text { level }(p)\end{array}$ \\
\hline \multicolumn{4}{c}{ NOVICES } \\
\hline Descending colon & $\mathbf{1 6 . 1 2}$ & 19.66 \\
\hline \multicolumn{4}{c}{ EXPERTS } \\
\hline Rectum & 87.14 & $\mathbf{3 3 . 4 0}$ & 0.04 \\
Sigmoid colon & 103.68 & $\mathbf{3 4 . 4 8}$ & 0.02 \\
Transverse colon & 225.48 & $\mathbf{2 1 . 7 2}$ & 0.02 \\
Hepatic flexure & 16.68 & $\mathbf{3 . 0 8}$ & 0.03 \\
Ascending colon & 14.80 & $\mathbf{4 . 4 8}$ & 0.01 \\
\hline
\end{tabular}

Table 8.1: Time comparison per colon segment (only significantly differing segments listed, lowest value formatted bold).

The significant results of the time analyses per segment are listed in Table 8.1. Novices were significantly faster during insertion through the descending colon with the robotic setup and assisting algorithm. Experts were significantly faster in many segments using the conventional method, but not in the descending colon and the splenic flexure. Novices found slightly more polyps (88.1\%, Q1-Q3 79.8 - 95.2\%) with the robotic method compared to using the conventional system (78.6\%, Q1-Q3 75.0 $91.7 \%$, non-significant). Experts found significantly more polyps using the conventional method with a median detection rate of 81.0\% (Q1-Q3 76.2 - 85.7\%) against 69.0\% (Q1Q3 61.0 - 75.0\%, $p=0.02$ ) when using the robotic method with ATC. The retraction times of the experts were within the range of $3.42 \mathrm{~min}-6.15 \mathrm{~min}$. One outlier was present, which was an expert using the conventional setup (> $8.5 \mathrm{~min}$ ). For novices, this range was $3.83 \mathrm{~min}-7.64 \mathrm{~min}$.

\subsection{Discussion}

In this study we evaluated the technical feasibility of an assisting automated lumen centralization algorithm implemented in a robotized colonoscopy setup. Our hypothesis was that the ATC would work in real-time in the robotized system it was implemented on, that it would predominantly be used in straight segments of the colon and that it would make endoscope introduction faster.

Clinically, we showed that novices were at least as efficient with the robotic system as with the conventional one, with a trend towards faster introduction. A significant difference was shown in a straight segment of the colon (descending colon). However, because of the low ATC use percentage, it is uncertain if this faster time is solely due to ATC use. Expert colonoscopists are fully trained using a conventional endoscope, and therefore it was expected that they are faster (TTC) using the conventional method. However, in both straight and curved segments, the robotic setup also obtained equal results compared to the conventional setup in terms of clinical efficiency. From this, 
we expect performance with the new system to improve with more training.

The system was developed to support endoscope insertion, and was expected to be easier and faster in this part of the procedure. Interestingly however, experts used ATC almost $12 \%$ of the time during the retraction phase of the procedure. When asked for their opinion, all expert users and some of the novices indicated that they experienced real additional value of the ATC functionality during retraction. It was considered more intuitive to centralize the lumen with the robotic system during the wall inspection for possible lesions than with the conventional system. Furthermore, all users indicated to see added value of the system for novice users, which is considered an excellent result.

Study results also revealed interesting comments on the visual cue that indicated the automatically found target direction. Several novice and expert users not only deemed the little white circle helpful, but stated they were following the cue even when they were not using the ATC function. This implies that the added functionality of the ATC algorithm may be partly established by visual assistance, and partly by autonomous correction of the tip.
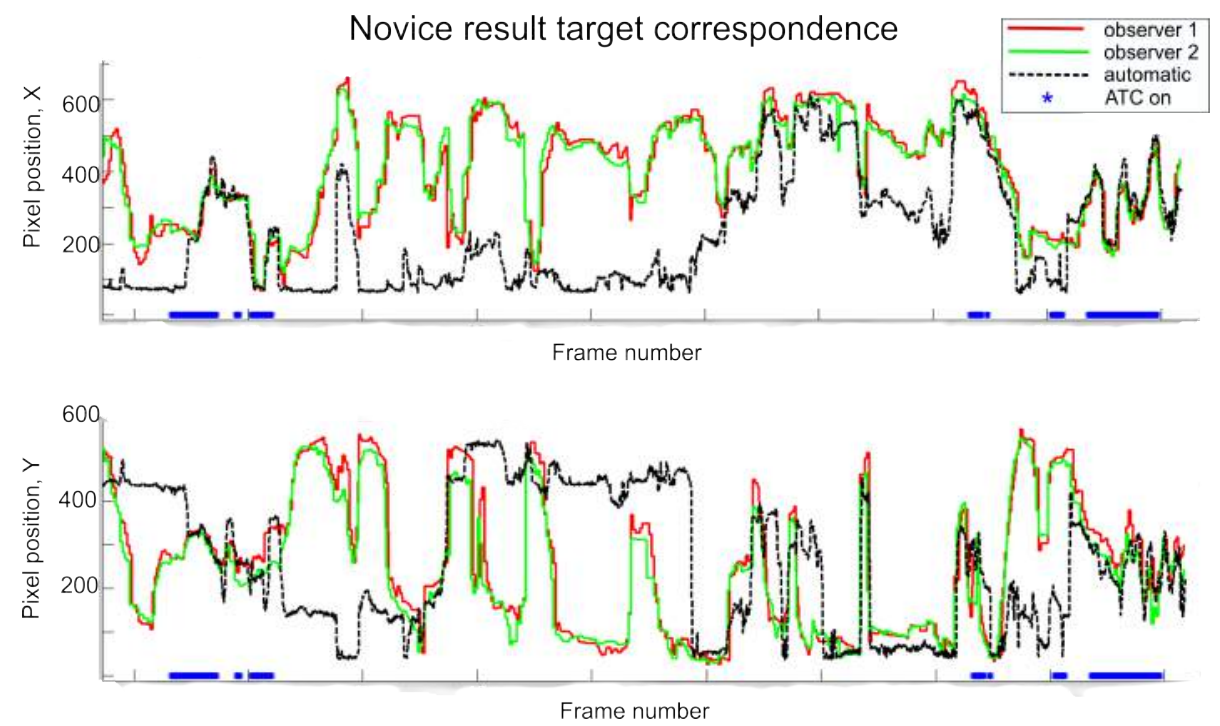

Figure 8.9: Example graph showing $X$ (top) and $Y$ (bottom) pixel coordinates of the found target in the image. The dashed line indicates the automatically found target, the others show the manually indicated target. The straight lines at the bottom of each graph indicate periods that ATC was turned on.

Experts not only used automatic lumen centralization more often (although nonsignificant), but also for a longer period of time $(p<0.001$, Figure 8.7 and Figure 8.8). Yet, the numbers overall are lower than expected. The low percentage of time ATC was used could theoretically be due to the low level of agreement of the users with 
the target location of the central lumen. Therefore, we compared the automatically found target location to the location that medical experts would steer the endoscope. Figure 8.9 illustrates the $X$ - and $Y$-coordinates of the found targets during part of a novices' introduction phase. The solid lines indicate manual indication of the target by expert observers, the dashed line shows the automatic result. If ATC is turned on the lines correspond well. However, there also are regions where the lines correspond well and ATC is not turned on. Therefore we think that lack of training with the system causes the low use of ATC, which should therefore be improved in a next study. The fact that the ATC is not always 'right' can be due to a number of factors, including the fact that the medical doctor would not always steer towards the central lumen. This is the reason the ATC functionality is designed in an assisting fashion.

Measuring clinical effectiveness was done through the polyp detection rate. Novices found an equal average amount of polyps in both modalities; experts found significantly more polyps using the conventional system. However, this measure was suffering from confounding factors, such as differing retraction times. We think that clinical effectiveness therefore cannot be established reliably from this outcome measure.

In terms of technical feasibility, the algorithm functions well and has helpful assisting functionality. A next study should be optimized specifically on the amount of training users need to perform equally with the new system and the conventional one.

\subsection{Conclusion}

In conclusion, the ATC algorithm combined with the Teleflex robotized endoscope setup works well in an experimental setup that closely resembles the clinical environment. The non-inferiority of this system is shown for novices, although ATC use was lower than expected. The relatively extensive use of ATC during the retraction phase of the procedure suggests an interesting added value of the system during this phase.

The next steps in this research include adjusting the system according to user feedback and an elaborate study on clinical effectiveness using thoroughly trained participants. The developed algorithm was tested in a colonoscopy simulation environment, but was not specifically designed for this. The algorithm can therefore be applied in any robotic flexible endoscopy system that is used in diagnosis of tubular organs.

\subsection{Acknowledgements}

We would like to thank all the test persons who voluntarily participated in this study: the students of technical medicine and the gastroenterologists. In addition, we thank the endoscopic department of the Meander Medical Center for their kind cooperation by lending materials and workspace.

\subsection{References}

Please refer to the bibliography at the end of this thesis. 


\title{
A real-time target tracking algorithm for robotized endoscopy
}

David Lynch

\begin{abstract}
Published as: Nanda van der Stap, Luuk Voskuilen, Guido de Jong, Hendrikus J.M. Pullens, Matthijs P. Schwartz, Ivo A.M.J. Broeders, \& Ferdi van der Heijden (2015). A Real-Time Target Tracking Algorithm for a Robotic Flexible Endoscopy Platform. 2nd workshop on CARE, MICCAI 2015, Munich, Germany.
\end{abstract}

Complex endoscopic interventions require a new generation of devices and instruments. A robotic platform for flexible endoscopy through telemanipulation was developed to meet this demand. The concept of telemanipulation allows the development of software for computer-aided surgery. Intelligent navigation such as automated target centralization could assist the endoscopist during procedures. A real-time algorithm was designed for tracking a target region that is of specific interest for the surgeon. Therefore, the physician needs to indicate the region to be tracked, which then will be centralized (locked). The goal of this research is to investigate the robustness and accuracy of the tracking algorithm during endoscopic interventions. 


\subsection{Introduction}

A trend towards minimizing the invasiveness of surgical procedures exists. Instead of open surgery, endoscopic or keyhole surgery is more often performed. Endoscopic surgery, where rigid cameras or endoscopes are used, decreases the amount of scarring and blood loss in the patient, leading to less pain and faster recovery times. With the transition from open to endoscopic surgery, flexible endoscopes are looked at for more complex procedures as well. Originally, only diagnostics and small therapeutic interventions (the removal of polyps in the colon for example) were performed with flexible endoscopes. Nowadays, increasingly large tumors are being removed, with the aim to spare the patient from a more invasive surgical procedure.

However, flexible endoscopes have vital drawbacks that make them difficult to handle, especially during complex procedures [16]. Endoscope handling is not intuitive and far from ergonomic. The instruments that can be inserted through the working channel of the endoscope are not capable of triangulation. Mostly, only one instrument channel is present, causing many instrument changes and a significant loss of dexterity.

Robotization is thought to improve the handling properties and dexterity of flexible endoscopes. Additionally, navigation can be automated (e.g. [45, 77, 131]). Our research is specifically aimed at automating endoscope navigation and fixation during interventions. Ultimate aim is to use image-based control to correct the endoscope tip once the endoscope is at the intervention site. The endoscopist can indicate a focus area manually, and the system described here will track this area continuously in the image. The tracked area will be kept in the center of the screen as much as possible, resulting in a so-called 'target lock'. The target should be centralized despite movement of the endoscope or the environment.

Others have investigated re-targeting of endoscopes. A useful application is for instance the optical biopsy, a visualization of cellular structures using optical instruments in the working channel of an endoscope. SLAM (Simultaneous Localization and Mapping) techniques combined with probe tracking, video manifolds for the patientspecific clustering of images and epipolar geometry recovery are examples of solutions for the retargeting problem in this application $[132,133,134,135]$. Chu et al. describe a flexible tip for a rigid endoscope and target tracking, but it is unclear which tracking approach they use [136].

We are interested in developing a clinically successful target locking system for robotized flexible endoscopy. This leads to the following requirements:

1. Real clinical added value: the procedure of interest should not be hindered or take longer due to the automation.

2. Robust to low texture frames, large movements, varying illumination conditions, instrument interference and occlusions (fluids, tissue deformation).

3. Accurate enough to enable small tip corrections in the order of a few millimeters.

4. Real-time functionality, such that the endoscopist can direct all his/her attention towards the surgical target instead of controlling the camera.

5. Easy correction functionality: if the target changes, for instance if tissue is taken from it, it should be easy to re-localize the target region. 
Feedback for the control will be obtained from the images by visual motion tracking and correcting for this. Motion tracking has been employed in flexible endoscopy [45]. A key issue is robustness and accuracy of tracking, implying an accurate outlier detection mechanism. Visual motion tracking is challenging due to the nature of the images (often low in texture and suffering from the artifacts named above in 2.). There is a trade-off between accuracy and computational effort with feature tracking algorithms. For our application, the algorithm must run fast enough to accurately correct the tip before the endoscopic image has changed too much.

The contribution of this paper lies in the real-time, accurate, feature-based target tracking aimed at optimal system performance with real clinical value. Therefore, real clinical data is used for evaluation. The described application is developed for the robotized flexible endoscopy system Teleflex [53], but minor changes can make it suitable for other robotized endoscopes.

\subsection{Materials and methods}

System requirements were established in close collaboration with several expert endoscopists. A thorough clinical evaluation among the endoscopists led to the conclusion that Routine colonoscopies, ERCPs and EMRs (Endoscopic Mucosal Resections) were the interventions most likely to benefit from a target lock. These procedures are known for their clinical complexity with respect to specific sub-interventions. The most difficult part during an ERCP is the insertion of a probe in Vater's papilla. Once the papilla is in the proper position in the view, the endoscope should remain fixed so that the probe can be manipulated properly. Similar situations were indicated for colonoscopies and EMRs. Twelve image sequences were selected and contained the various sub-interventions of interest for the target lock (Table 9.1). A sub-intervention was estimated to last for 4 seconds on average; this is therefore the length of the sequences. An exception forms the papilla insertion during an ERCP. Therefore, the length of sequence 3 was doubled.

To use images as control feedback, the bandwidth of the motion should be an order of magnitude less than the sample frequency. The Nyquist frequency for $25 \mathrm{fps}$ is 12.5 $\mathrm{Hz}$. For control purposes, the rule of thumb is to have a 5-10 times higher sample frequency than the motion bandwidth. In this case, most motions have a frequency of $0-5 \mathrm{~Hz}$. With an effective frame rate (sample frequency) of 24-25 fps this requirement was met in our system.

Real-time optimization can be done by cropping or down-scaling of the images, frame-skipping, code- and platform-optimizations or heterogeneous computing. The latter will result in the most significant improvement without data loss. To enable heterogeneous computing, the feature tracking algorithms were implemented using OpenCV with OpenCL.

Image sequences had a resolution of $768 \times 576$ and a frame rate of 25 frames per second (fps). All results were generated using a HP Elitebook 8570 w mobile workstation running on a 64 bit operating system (Windows 8.1) with an Intel Core i7-3630QM processor, 8GB DDR3 RAM and an AMD FirePro M4000 graphics card. Programming 


\begin{tabular}{|c|c|c|c|c|}
\hline Number & Procedure & Intervention & Target & Disturbing factor \\
\hline 1 & Colonoscopy & Polypectomy & Polyp & Coarse movements \\
\hline 2 & Colonoscopy & Polypectomy & Polyp & $\begin{array}{l}\text { Poor illumination; Occlu- } \\
\text { sions }\end{array}$ \\
\hline 3 & ERCP & Cannulation & $\begin{array}{l}\text { Vater's } \\
\text { ampulla }\end{array}$ & $\begin{array}{l}\text { Target near edge of the } \\
\text { FOV; Occlusions }\end{array}$ \\
\hline 4 & ERCP & Sphincterotomy & $\begin{array}{l}\text { Vater's } \\
\text { ampulla }\end{array}$ & $\begin{array}{l}\text { Target near edge of FOV; } \\
\text { Smoke; Occlusions }\end{array}$ \\
\hline 5 & EMR & Injection & $\begin{array}{l}\text { Primary } \\
\text { tumor }\end{array}$ & 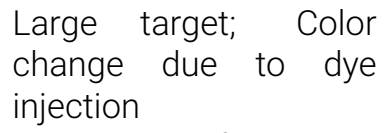 \\
\hline 6 & EMR & APC & $\begin{array}{l}\text { Residual } \\
\text { lesion }\end{array}$ & $\begin{array}{l}\text { Large area of removed } \\
\text { mucosa; Small target; } \\
\text { Sparks }\end{array}$ \\
\hline 7 & Colonoscopy & Injection & Polyp & Color change due to dye \\
\hline 8 & Colonoscopy & Polypectomy & Polyp & Large polyp; Dirt on lens \\
\hline 9 & ERCP & Cannulation & $\begin{array}{l}\text { Vater's } \\
\text { ampulla }\end{array}$ & Endoscope motion \\
\hline 10 & ERCP & Stent removal & $\begin{array}{l}\text { Vater's } \\
\text { ampulla }\end{array}$ & $\begin{array}{l}\text { Multiple instruments in } \\
\text { view }\end{array}$ \\
\hline 11 & EMR & $\begin{array}{l}\text { Partial resec- } \\
\text { tion }\end{array}$ & $\begin{array}{l}\text { Primary } \\
\text { tumor }\end{array}$ & $\begin{array}{l}\text { Large target; Coarse } \\
\text { movements; Dye injec- } \\
\text { tion }\end{array}$ \\
\hline 12 & Colonoscopy & APC & Polyp & $\begin{array}{l}\text { Sparks; Bubbles; Small } \\
\text { target }\end{array}$ \\
\hline
\end{tabular}

Table 9.1: Image sequence properties. APC: Argon Plasma Coagulation. Note: in all sequences, instruments are present in the field of view (FOV).

was done using Microsoft Visual Studio Express 2013, with libraries from OpenCV 2.4.9 and OpenCL 1.1. A colonoscope, an ERCP-scope and a pediatric colonoscope (for EMR) were used to record the procedures. The properties of each of them are listed in Table 9.2 .

\subsubsection{Algorithm}

For accurate and robust feature tracking, SIFT (Scale Invariant Feature Transform [92]) will be suitable, because blob-like features are abundantly present in the image sequences that were used (Figure 9.1). However, detecting and matching these features takes considerable computational effort. For our real-time application, we therefore chose to use SURF features (Speeded-Up Robust Features [69]). These are nearly as accurate as SIFT features, but decrease computational effort considerably [137]. As stated in the Introduction, an accurate outlier detection mechanism is key to robust system performance. In the original SURF algorithm brute force matching was se- 


\begin{tabular}{l||ll} 
Procedure & Endoscope & Properties \\
\hline Colonoscopy & Olympus CF-H180AL & FOV: 170; DoF: 2-100 mm; Length: \\
& & $1680 \mathrm{~mm}$. \\
ERCP & Olympus TJF-160VR & FOV: $100^{\circ} ;$ DoF: $5-60 \mathrm{~mm}$; Length: \\
& & $1240 \mathrm{~mm}$. \\
EMR & Olympus PCF-PH190L & FOV: $140^{\circ} ;$ DoF: 2-100 mm; Length: \\
& & $1680 \mathrm{~mm}$. \\
\hline
\end{tabular}

Table 9.2: Properties of each endoscope used to record the image sequences. FOV: Field of View. DoF: Depth of Field. DoV: Direction of View.

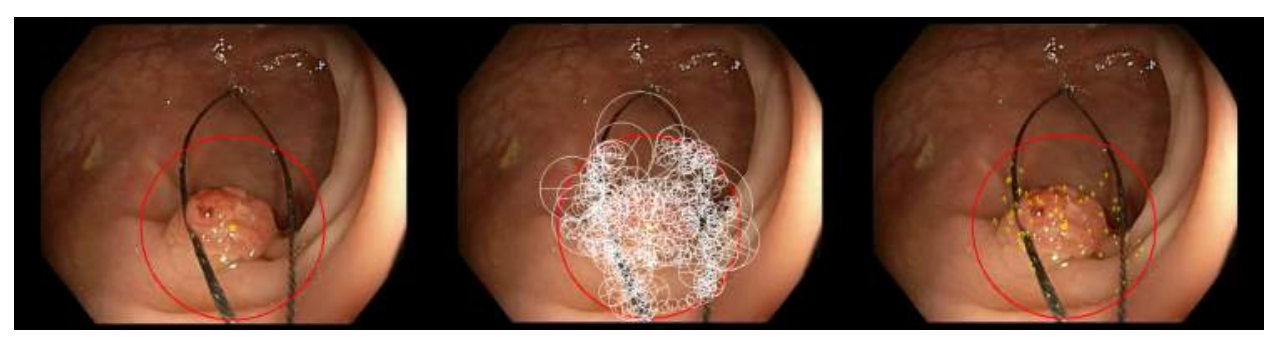

Figure 9.1: Example image of polypectomy with polyp and instrument present. Left: scene with ROI indication. Middle: SURF features (indicated as white circles). Right: final motion vectors (indicated as arrows).

lected for accuracy reasons. In an attempt to reduce computational time and improve reliability of the matches, we added the second-to-nearest-neighbor (SNN) distance ratio check, as proposed by Lowe [92]. To increase the number of features, even in low-textured areas, preprocessing (grayscale conversion and histogram equalization) was applied on the images. Outlier removal based on vector magnitude was added to the algorithm to improve robustness. The complete algorithm works as follows:

1. Initialization:

(a) Acquire and visualize the first image, reference image $I_{r e f}$.

(b) Select the target ROI in $I_{r e f}$ : a circle with position $\mathbf{x}_{r e f}$ and radius $R$.

(c) Set $\mathbf{x}_{\text {target }}=\mathbf{x}_{\text {ref }}$.

(d) $\left\{\mathbf{y}_{\text {ref }}(n), \mathbf{f}_{\text {ref }}(n)\right\}=$ get_surf_features $\left(I_{\text {ref }}, \mathbf{x}_{r e f}\right)$.

2. Acquire current image $I_{\text {cur }}$.

3. $\left\{\mathbf{y}_{\text {cur }}(m), \mathbf{f}_{\text {cur }}(m)\right\}=$ get_surf_features $\left(I_{\text {cur }}, \mathbf{x}_{\text {target }}\right)$.

4. Match $\left\{\mathbf{f}_{r e f}(n)\right\}$ to $\left\{\mathbf{f}_{\text {cur }}(m)\right\}$ with SNN distance ratio check, yielding matched indices $\{n(k), m(k)\}$, with $k=1, \ldots, K$. 
5. Get displacement vectors $\left\{\mathbf{d}(k)=\mathbf{y}_{\text {cur }}(m(k))-\mathbf{y}_{\text {ref }}(n(k))\right\}$

6. Remove outliers. Condition: $\|\mathbf{d}(k)\|>2 *$ median $(\{\|\mathbf{d}(k)\|\})$.

7. $\mathbf{x}_{\text {target }}=\mathbf{x}_{\text {ref }}+$ mean $(\{\mathbf{d}(k)\})$.

8. Repeat till end from 2.

Procedure $\{\mathbf{y}(n), \mathbf{f}(n)\}=$ get_surf_features $(I, \mathbf{x})$

1. Convert image $I$ from RGB to grayscale.

2. Apply histogram equalization to $I$ to increase feature number.

3. Detect SURF key point positions $\{\mathbf{y}(n)\}$ and key point descriptors $\{\mathbf{f}(n)\}$.

\subsubsection{Analysis}

Targets to be tracked (Table 9.1) were manually annotated throughout the image sequences by an expert interventional endoscopist (> 2000 endoscopies). The automatically found location was compared for accuracy to the manual results using intra-class correlation analysis (ICC, [67]). Tracking error was given by the Root Mean Square Error (RMSE) of the distance in pixels between the two targets. Computational times were recorded to measure real-time performance of the system. Robustness was measured by counting the number of feature matches and inlying matches per frame.

\subsection{Results}

In all sequences, the manually indicated target could be tracked with an high accuracy of 91.6\% (Q1-Q3 77.7\% - 99.0\%, see Table 9.3). Sequence 7 had the best tracking results with a correlation of $99.9 \%$ and a RMSE of 9.4 pixels. Median tracking error was 38.9 pixels (Q1 - Q3: 28.7 - 76.3, see Table 9.3). The algorithm proves robust against smoke, fluid and instrument interference, color changes, occlusions and poor illumination. Sequences that suffered from these artifacts nonetheless led to the best results (Figure 9.2). The text boxes and show that large motions and motion blur are the cause for the biggest tracking errors.

The median number of matches and inliers per frame was at most 239 and 73, respectively (Table 9.3). Note that the lowest number of matches and inliers correspond to the lowest ICC. Our matching approach was more accurate and slightly faster than the original brute force matching, with an average of $43.79( \pm 4.37)$ ms against 48.55 $( \pm 4.33)$ ms per frame. We have uploaded the image sequences in the additional conference materials to illustrate the disturbing factors more clearly.

\subsection{Discussion}

In this study, the accuracy and robustness of a real-time tracking algorithm for automated target centralization in a robotized flexible endoscopy system was evaluated. 


\begin{tabular}{c||llll} 
Sequence & ICC (\%) & $\begin{array}{l}\text { Median } \\
\text { matches }\end{array}$ & $\begin{array}{l}\text { Median } \\
\text { inliers }\end{array}$ & RMSE (pixels) \\
\hline $\mathbf{1}$ & 81.9 & 77 & 34 & 149.1 \\
$\mathbf{2}$ & 89.4 & 165 & 56 & 32.4 \\
$\mathbf{3}$ & 99.7 & 154 & 67 & 25.5 \\
$\mathbf{4}$ & 98.8 & 123 & 32 & 34.2 \\
$\mathbf{5}$ & 96.6 & 201 & 73 & 27.4 \\
$\mathbf{6}$ & 93.8 & 239 & 73 & 28.0 \\
$\mathbf{7}$ & 99.9 & 184 & 62 & 9.4 \\
$\mathbf{8}$ & 73.6 & 205 & 61 & 116.0 \\
$\mathbf{9}$ & 99.8 & 88 & 32 & 16.6 \\
$\mathbf{1 0}$ & 75.7 & 236 & 38 & 43.7 \\
$\mathbf{1 1}$ & 78.4 & 131 & 24 & 123.0 \\
$\mathbf{1 2}$ & 63.5 & 223 & 58 & 57.1 \\
\hline
\end{tabular}

Table 9.3: Results per image sequence.
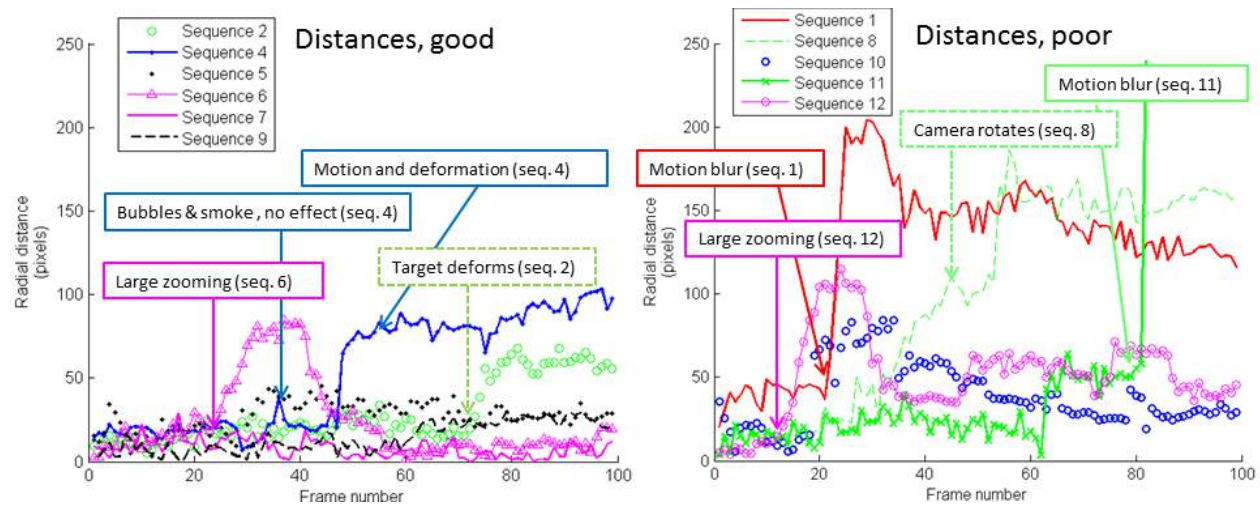

Figure 9.2: Left: Sequences with best ICC and smallest RMSE. Graphs show distance in pixels between the center of the two (automatic and annotated) targets. The text boxes explain the larger shifts. Right: Sequences with the poorest outcome. All tracking errors are caused by large motions.

This algorithm can be used for a variety of interventions. Here, we evaluated the algorithm using six image sequences of three different interventions. The achieved median accuracy was $91.6 \%$, which is an excellent result.

Robustness of the algorithm was shown by the continuous ability to track the target throughout the sequences, independent from procedure or tissue type, although several disturbing factors were present (Table 9.1). Inlying vectors mostly remained present and tracking was kept accurate, even with occlusions, color and illumination changes, surgical instruments, smoke and fluids present. Large and fast movements 
still form a problem; this caused most errors. If such an error occurred, the tracking was disturbed. For longer tracking periods of the same region this means reinitialization of the algorithm in its current form is sometimes necessary. However, we expect robustness to be improved with system implementation (see below).

Computations took a mean total time of $43.79 \mathrm{~ms}$ per frame. The algorithm could theoretically track the target every $\frac{1 \mathrm{sec}}{25 \mathrm{frames}}=40 \mathrm{~ms}$. However, when using a newer computer with a better CPU (Intel Core i5-3570K) and a better GPU (AMD Sapphire TriX R9 290), computational time was below $40 \mathrm{~ms}$ and real-time system performance was ensured.

A limitation of this research is that zooming motion is assumed to be absent, although small zooming motions were present in the used sequences. Therefore, our current focus is on the implementation of the algorithm in the robotic system, complete with zooming functionality.

Current research further includes optimizing the robotic control based on the feedback that is generated from this algorithm. When combining the algorithm with the robotic control, large tracking errors (such as these over 100 pixels) will be diminished by the integral action that is present in the robotic controller. This action effectively smoothes the feedback signal because of the limited displacement possibility of the motors within a certain time frame. If this smoothing will not be enough to obtain the desired robustness, smart filtering with which previous information (key frames) is employed will be added to the system.

Finally, we will focus on establishing clinical relevance and patient safety. The algorithms are integrated in the Teleflex system, which is currently being evaluated in a phase II clinical trial, and we expect good results from this evaluation.

\subsection{Conclusion}

A target lock was designed for complex flexible endoscopic interventions. The algorithm performed accurately, robustly and worked in real-time. Intelligent navigation in robotized systems could assist the endoscopist during complex and time-consuming procedures. Clinical added value for the patient still needs to be objectively evaluated, but preliminary evaluation results seem promising.

\subsection{References}

Please refer to the bibliography at the end of this thesis. 


\title{
A camera stabilization system for flexible endoscopic interventions
}

\author{
'He had them as spellbound as a \\ room full of Ewoks listening to \\ C-3PO'
}

Cory Doctorow

Preliminary results of the implementation and experimental validation of the system presented in Chapter 9.

This chapter contains the technological in-vitro evaluation results of the implemented target lock described in Chapter 9. The evaluation was performed using a KUKA arm and an artificial target and can be seen as a proof of concept of the system. The system as a whole is referred to as a camera stabilization system. 


\subsection{Introduction}

Robotizing endoscopes and using image-based navigation is currently researched in different forms (e.g. $[138,11])$. Two main approaches can be discerned. Either a completely new endoscopic system including a robotized endoscope is being developed (e.g. [139]), or currently used endoscopes in the clinic are robotized with robotic addons (e.g. $[83,53])$. The advantages of the former are that undesired properties of current flexible endoscopes, such as the difficulty to control them due to the flexibility, are avoided. However, this approach also leads to more expensive systems that are unlikely to be adopted quickly in medical practice. The second approach will lead to a cheaper system which can easily be incorporated in the current clinical workflow.

A disadvantage of this approach is that it is technologically more challenging. However, fulfilling the clinical need is key to developing successful technology in this area of research, and we believe that robotizing currently used flexible endoscopes is more advantageous here. Clinical needs in this area can be categorized in needs for diagnostics and needs for interventional endoscopy. We have proposed solutions for diagnostic flexible endoscopy earlier $[53,45,102]$. In the current research, we focus on unsolved clinical needs during flexible endoscopic interventions.

Problems that are currently encountered during interventions with flexible endoscopes are mainly related to the difficult steering mechanism. Using the turning knobs on the handle of the endoscope, the tip can be controlled (recall Figure 2.1 or Figure 5.1). However, since the camera is located at the tip and the instruments emerge from the working channel at the same tip, two complications arise:

1. The camera and instruments are parallel with respect to each other;

2. If the camera moves, the instrument moves, and vice versa.

A solution for the first problem was found in robotization of the instruments, as demonstrated for instance in $[53,9]$. A possible solution for the second problem may be found in automated stabilization of the endoscope. If stabilization is automated, the endoscopist will have his or her hands free for instrument control. Endoscope stabilization can be achieved through image-based control. Image-based control has the advantage of not only being adaptable to unpredictable environments, but also of being able to handle different tissue structures.

The camera of the endoscope is located at the tip, so if the camera motion is compensated for, the endoscope is stabilized. To develop a stabilization system, the motion of the camera with respect to the structure of interest needs to be automatically tracked. However, organ deformation, endoscope flexibility and environmental factors such as smoke and fluids may cause errors in the tracking and centralizing of the anatomical target. Other researchers have focused on developing a tracking algorithm before. However, either the goal was not to implement the tracking algorithm in a robotic system (e.g. [140, 141, 142]), or a rigid endoscope was used instead of a flexible one (e.g. [143]).

We have described a tracking algorithm earlier, specifically suited for our application [101]. This algorithm is able to accurately track camera motion during different 


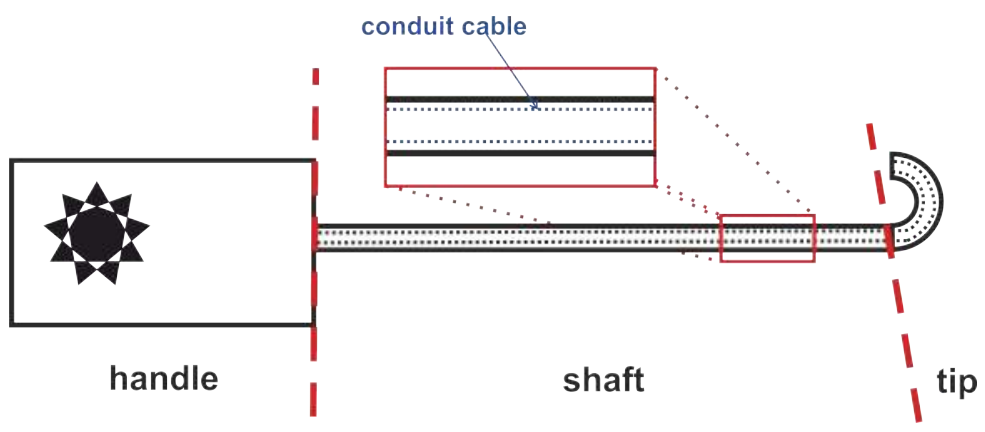

Figure 10.1: The endoscope is schematically divided in three parts to explain the working mechanism. In the handle, turning knobs cause conduit cable displacement. The cables in the shaft run through a plastic sheet. The tip is the endoscope part that can be controlled by the robotic system.

in-vivo interventions in real-time. Although accurate tracking forms the basis for camera stabilization, only with accurate control can such a system be successful. In the current chapter, we describe a proof of concept for the complete camera stabilization system for flexible endoscopic interventions. We implemented the described tracking algorithm on a robotized flexible endoscopy platform and evaluated its performance.

\subsubsection{System requirements}

During therapeutic interventions the endoscopist needs to have immediate feedback of the circumstances at the endoscope tip site. The endoscope needs to respond directly. Real-time functionality of the system and compensation for undesired flexible behavior are therefore vital. 'Real-time' is defined here as a system that provides feedback to the endoscopist fast enough to not limit the workflow. In practice, the workflow speed depends on task complexity (which influences reaction time of the surgeon) [144] as well as possible motor response to a visual stimulus [145]. About task complexity no quantitative requirement is possible since this differs per task. However, a qualitative requirement here is that the system may not change the current clinical workflow unacceptably.

Regarding the motor response a quantitative requirement is possible. The minimum time a visual stimulus needs to be visible to be able to respond to it, is shown to be $100 \mathrm{~ms}$ [145]. This means in our system, a refresh rate of the target positioning information for the endoscopist of 10 times per second is required.

Furthermore, the tip control needs to be accurate enough to enable small corrections during therapeutic interventions. A second requirement is that adjustments of a few millimeters need to be made reliably during these procedures. Why this requirement does not have a straightforward solution will be explained in the next section. 


\subsubsection{Background and related work}

For accurate tip control some knowledge is needed about dynamical behavior of endoscopes. Endoscopes are cable-driven manipulators, which are actuated by conduit cables that run through plastic tubes or sheets (Figure 10.1). One of the biggest problems all researchers encounter when trying to robotically control endoscopes, is the complex non-linear dynamical behavior they exhibit. A setup was designed earlier for visual position measurements of multiple endoscope tips (submitted work, [146]). The setup is shown in Figure 10.2. In the experiments performed with this setup, the angles of the steering knobs at the endoscope handle were increased back and forth using the electro motors. The difference between motor displacement and actual tip displacement subsequently resulted in figures comparable to Figure 10.3.

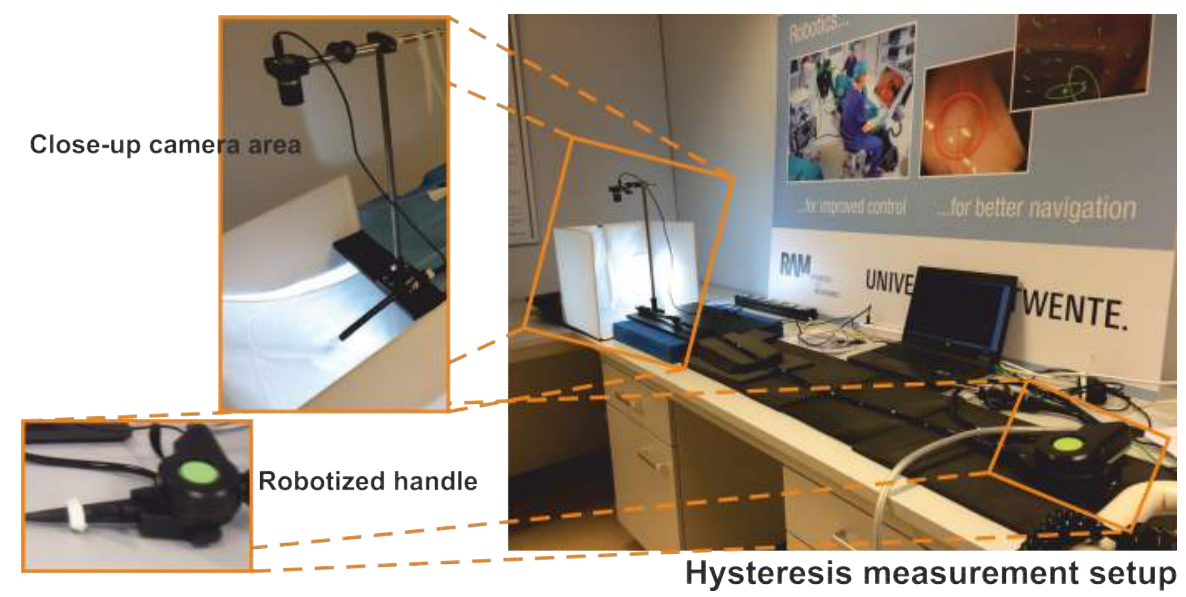

Figure 10.2: The hysteresis in the endoscope was measured using the robotic system, a camera on the outside of the tip and a quantification algorithm based on vision techniques.

The results of the experiments showed that hysteresis is present in the endoscopic motion. Hysteresis shows when the wheels on the control handle are changed in the opposite direction from where they were previously going (plateau values at the top and bottom of the graph), but also occurs when the endoscope lies straight and has to 'start' moving (middle plateau values). The causes for this phenomenon likely are slackness of the conduit cables, as well as internal friction forces. The amount of slackness depends on the (amount of) use, age or handling of the endoscope, and can only be changed by maintenance by the manufacturer.

The conventional approach to solve problems with hysteresis is to perform an exact hysteresis identification and subsequently try to compensate for it using feedforward compensation on the control $[147,148,131,149]$. This is also why others have tried to quantify the nonlinear effects (e.g. [150, 151, 152]). Most endoscope models are based on the main assumptions of no cable slack (pretension) or no external disturbances being present. Moreover, even if these effects are taken into account, an identification 


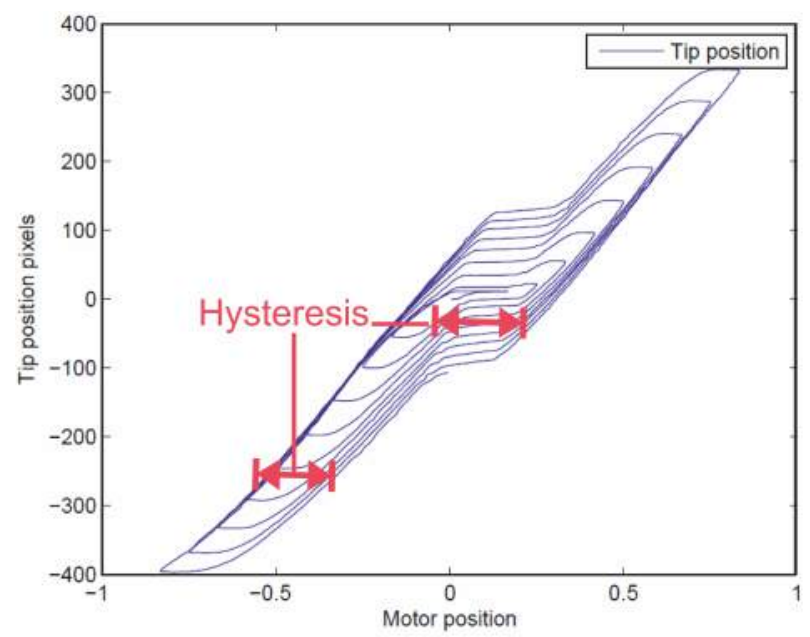

Figure 10.3: Resulting plot from position measurements. When the motor position and the tip position are plotted against each other, hysteresis shows.

will have to be done before every procedure in which the endoscope is used for this approach to work in practice. The measurements with our setup showed hysteresis of endoscopes changes per endoscope and over time for each individual endoscope [146]. The reason we found nonlinear effects that are more unpredictable than are described by others is probably that we used real endoscopes and not a custom-made measurement setup. Performing an exact hysteresis identification procedure before each endoscopy is not a feasible scenario within the clinical workflow and therefore this solution does not fit our requirements.

Predicting the hysteresis based on other sensor information, such as motor position and camera movement, is a possible solution for our system. In [147], hysteresis is also predicted using camera displacement information. In addition, the authors employ 'physiological motion rejection' to stabilize the camera. They use measurements from the artificial ventilation equipment as input for the motion prediction. If the prediction does not match the camera motion, the error is attributed to hysteresis effects. Unfortunately in our application patients are not ventilated, which means we cannot employ this information or the assumption of a periodical and constant motion. In addition, we question the need for breathing motion rejection in our application. First, many surgical areas of interest are not or in very small amount subject to breathing motion disturbance. Second, the authors use the images as an input for the hysteresis estimation after breathing motion correction. Yet, as the authors state in [147], 'physiological motions and self-motion of the endoscope can hardly be separated' using their technique, introducing a source of error. We think it is necessary to make the endoscope follow the target tissue regardless of the nature of the present movement. The robustness of the tracking algorithm is therefore the key to success. 
In addition, the motor position in combination with a kinematic model of the endoscope then can predict the hysteresis of the endoscope. Instead of identifying the hysteresis exactly, we thus start by predicting the hysteresis assuming general parameters and always apply a compensation for it when the direction of the endoscope changes or when the tip changes from the straight to the bent position.

\subsubsection{Contributions}

In the current chapter the main contribution is the proof of concept of a flexible endoscopic camera stabilization system. This system works in real-time and does not disturb clinical workflow severely.

\subsection{Methods and materials}

Although the idea of creating a virtual link between an endoscope tip and an anatomical target is not new, the current approach to solve this problem is. Novelty mostly lies in the application to currently used flexible endoscopes and in the algorithm's implementation within the Teleflex robotic system [53].

In the remainder of this section, first the approach to accurate tip control will be described (section: Hysteresis prediction). This is followed by the description of the experimental evaluation of the system.

\subsubsection{Hysteresis prediction}

A system requirement is that tip control needs to achieve a few millimeters accuracy. Therefore, a basic hysteresis prediction was designed and implemented as a feedforward compensation. The compensation is based on a relatively simple model following from Figure 10.1. The conduit cables that steer the endoscope tip go through the flexible shaft. The discrepancy between motor position and expected tip position (hysteresis) is modeled to be caused by Coulomb friction along the cables, as well as the compliance of the cables themselves. In addition, friction is modeled to exist between the bending segments in the tip.

From Figure 10.4, obtained through the earlier described measurements, can be seen that performing an hysteresis measurement with the tip straight (Figure 10.4A) differs significantly from one with the tip bent (Figure 10.4B). A bent tip means that the tip is constantly bent in one direction (one cable is pulled) when the other cable is pulled and loosened to move the tip in the other direction. From these results we concluded that friction between the cables must also be present, and this was therefore implemented in the model.

The hysteresis in the middle area of the graphs is still unaccounted for. In our model we assumed this hysteresis is due to the material memory present in the outside wrapping of the tip. Many polymers have a 'stress/strain hysteresis' property, which means that energy is lost when the material is stretched out and it will not fully return to its original stiffness. The tip is therefore modeled as a rotational spring with a stiffness that depends on the previous maximum bending angle of the tip. 

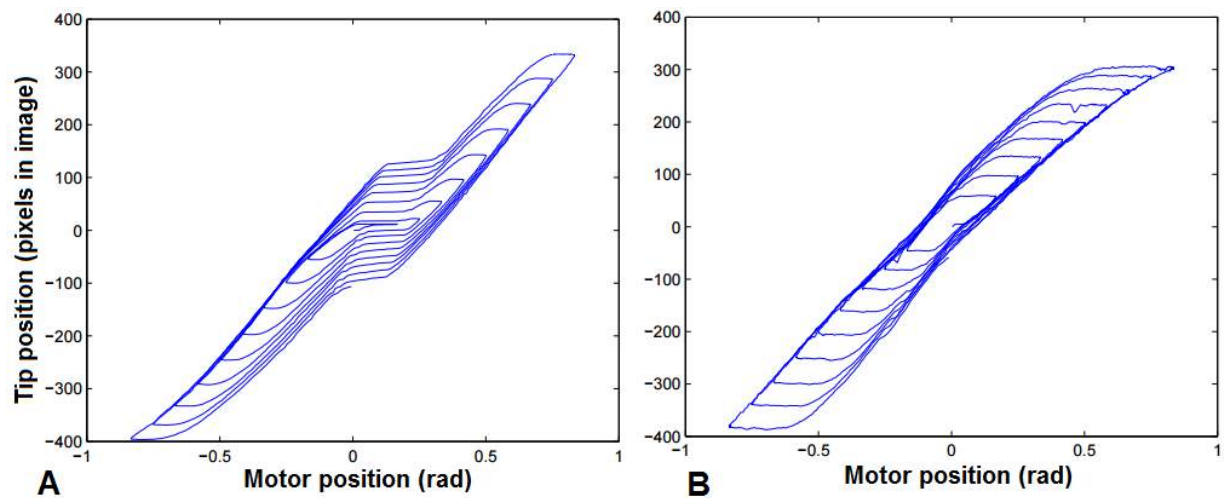

Figure 10.4: A: Measurement with straight tip. B: tip bent in one direction, pulled in the other. Observe that the deadband is almost zero, and the width of the hysteresis differs.

\subsubsection{Experimental evaluation method and materials}

Goal of the experimental evaluation was to track the motion of the endoscope tip externally and relate this to the also externally measured target motion. Optical tracking was used in the form of the OptiTrack system (Optitrack, NaturalPoint Inc., Corvallis, OR, USA). This system employs small spherical reflectors to accurately assess an object's position (Figure 10.5C). Custom-made reflector bearers were designed using rapid prototyping techniques (Figure 10.5A and B).
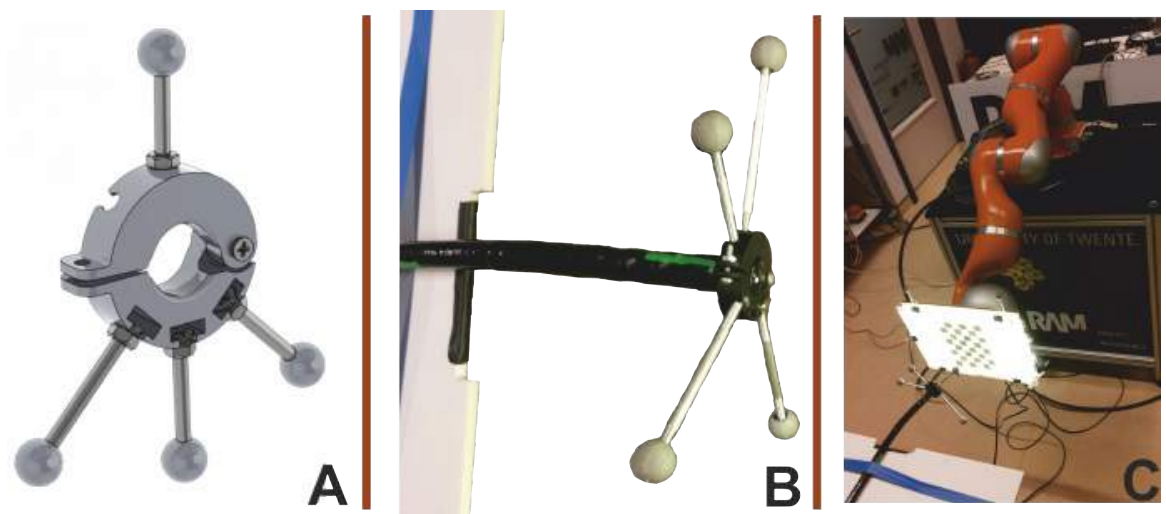

Figure 10.5: An optical tracking system with reflecting spheres is used for accurate endoscope positioning. A \& B: The custom-made reflector holder, schematic and on the endoscope. C: The reflectors in the complete setup.

In addition, we attached an artificial target to a KUKA lightweight robotic arm (Fig- 
ure 10.5C). This way, the true motion of the target with respect to the world was known from the encoders in the arm. The OptiTrack measurements of the subsequent endoscope movements then provided insight in the system response as a whole. Frontal illumination of the target led to specular reflections on the paper which influenced the tracking performance. Therefore, the target was placed in front of a LED-system which as a whole was stabilized by the robotic arm (Figure 10.6).

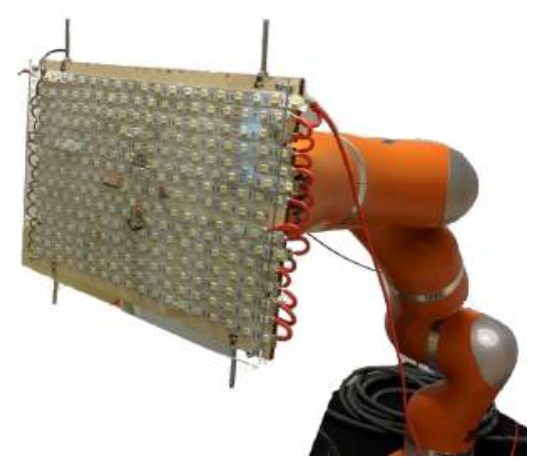

Figure 10.6: A target bearer was designed with which the target could be illuminated from the rear, so specular reflection artifacts were prevented.

\section{Materials}

For the experiments a flexible colonoscope (CF-H180AL, Olympus Corporation, Tokyo, Japan) connected to a video processor (Evis Exera II, Olympus Corporation, Tokyo, Japan) were used for image acquisition. The image-based control was performed on a HP EliteBook 8570w (Windows 8) using Python 2.4 and OpenCV 2.7.

\section{Analysis and measurements}

The goal of the measurements was to evaluate the tracking and control performance of the system. For tracking performance evaluation, the tracked target by the camera was compared to the ground truth motion of the target as performed by the robotic arm.

The comparison was established as follows. Let the coordinate frame of the reflector bearer on the tip of the endoscope be $\psi_{r}$, the coordinate frame of the reflector bearers on the target plate $\psi_{t}$ and let the camera frame be $\psi_{c}$. The motion of the target with respect to and expressed in the camera frame (and detected by the camera) needs to correspond to the motion of the target with respect to the reflector bearers, since these are observed by the OptiTrack system (and not the camera itself). The transformation between the reflector bearers and the camera (i.e. $\psi_{\mathbf{c}} \mathbf{H}_{\psi_{\mathbf{r}^{\prime}}}, \psi_{\mathbf{c}} \mathbf{H}_{\psi_{\mathbf{t}}}$ and $\psi_{\mathrm{t}} \mathbf{H}_{\psi_{\mathrm{r}}}$ ) needs to be known to be able to compare them:

$$
\psi_{\mathrm{c}} \mathbf{H}_{\psi_{\mathrm{t}}}^{\psi_{\mathrm{c}}} \cong \psi_{\mathrm{c}} \mathbf{H}_{\psi_{\mathrm{t}}}^{\psi_{\mathrm{o}}}
$$




\begin{tabular}{l||lllll} 
No. & Movement & Direction & Speed & Zoom & Tip control \\
\hline $\mathbf{1}$ & Periodical & Horizontal & Increasing & Off & Off \\
$\mathbf{2}$ & Periodical & Vertical & Increasing & Off & Off \\
$\mathbf{3}$ & Periodical & Circular & Increasing & Off & Off \\
$\mathbf{4}$ & Periodical & Horizontal & Constant & Off & On \\
$\mathbf{5}$ & Step & Horizontal & Constant & Off & On \\
\hline
\end{tabular}

Table 10.1: Properties of the different measurements that were done to evaluate system performance.

Here, $\psi_{\mathbf{c}} \mathbf{H}_{\psi_{\mathrm{t}}}^{\psi_{\mathrm{c}}}$ is the configuration of frame $\psi_{t}$ with respect to $\psi_{c}$, expressed in $\psi_{c}$. The same goes for $\psi_{\mathbf{c}} \mathbf{H}_{\psi_{\mathbf{t}}}^{\psi_{\mathrm{o}}}$, but then it is expressed in the OptiTrack coordinate frame $\psi_{0}$.

A camera calibration procedure was performed to obtain the tracked target pixel points in 3D camera coordinates. Following [105], the target points $\mathbf{x}_{\psi_{\mathbf{t}}}$ in homogeneous coordinates can be expressed in $\psi_{\mathbf{c}}$ using:

$$
\hat{\mathbf{x}}_{\psi_{c}}=\mathbf{K}^{-1} \hat{\mathbf{x}}_{\psi_{t}}
$$

$\mathbf{K}$ is the camera matrix, containing intrinsic camera properties such as the principal point. From this, the transformation $\psi_{\mathbf{c}} \mathbf{H}_{\psi_{\mathrm{t}}}^{\psi_{\mathrm{c}}}$ can be obtained using the dimensional knowledge of the checker board that was used during calibration. The transformation $\psi_{\mathrm{c}} \mathbf{H}_{\psi_{\mathrm{t}}}^{\psi_{\mathrm{o}}}$ is computed through:

$$
\psi_{\mathrm{c}} \mathbf{H}_{\psi_{\mathrm{t}}}^{\psi_{\mathrm{o}}}=\psi_{\mathrm{c}} \mathbf{H}_{\psi_{\mathrm{r}}} \psi_{\mathrm{r}} \mathbf{H}_{\psi_{\mathrm{t}}}
$$

Both $\psi_{\mathrm{c}} \mathbf{H}_{\psi_{\mathrm{r}}}$ and ${ }_{\mathrm{r}} \mathbf{H}_{\psi_{\mathrm{t}}}$ are measured by the OptiTrack system through the reflectors on the target and the endoscope tip.

Different measurements with different aims were done using the described setup (Table 10.1). We were interested in the outcome for two main end points: the tracking performance and the hysteresis compensation performance. To evaluate the tracking performance, the endoscope was measured lying straight and no active robotic control of the steering knobs was turned on. The target then moved with respect to the image center, and the physical target displacement was tracked and compared to the image tracking results.

For hysteresis compensation performance evaluation, the endoscope was put in a straight or a bent position under angle $\omega$. The target plate was then translated perpendicular to the endoscope tip (Figure 10.7). The target was selected to be on $\frac{1}{4}$ of the image width and $\frac{1}{2}$ of the image height. The correcting motion of the system can be considered a step response here. Parameters of interest were accuracy of the system response and the system latency.

The next part of the measurements consisted of complicating the target motion and measuring system response for multiple effects. The robotic arm was programmed to move the target plate circular, which should result in situations in which the system 


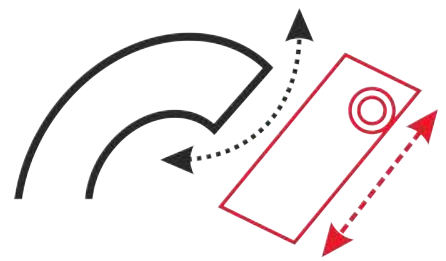

Bent tip measurement

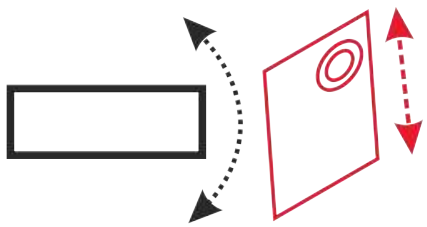

Straight tip measurement

Figure 10.7: Hysteresis compensation evaluation was done with the endoscope tip bent and straight. The target plate was moved perpendicular to the endoscope tip.

has difficulty with performance (robustness of tracking, accuracy of hysteresis compensation).

\subsection{Results}

\subsubsection{Hysteresis compensation}

Simulations were done to check whether the assumptions in our model were realistic. First, the simulated motor position was plotted against the simulated tip position upon a sinusoidal input (Figure 10.8). The positions of the deadband and hysteresis corresponds to the earlier performed measurements in real endoscopes (recall Figure 10.3).

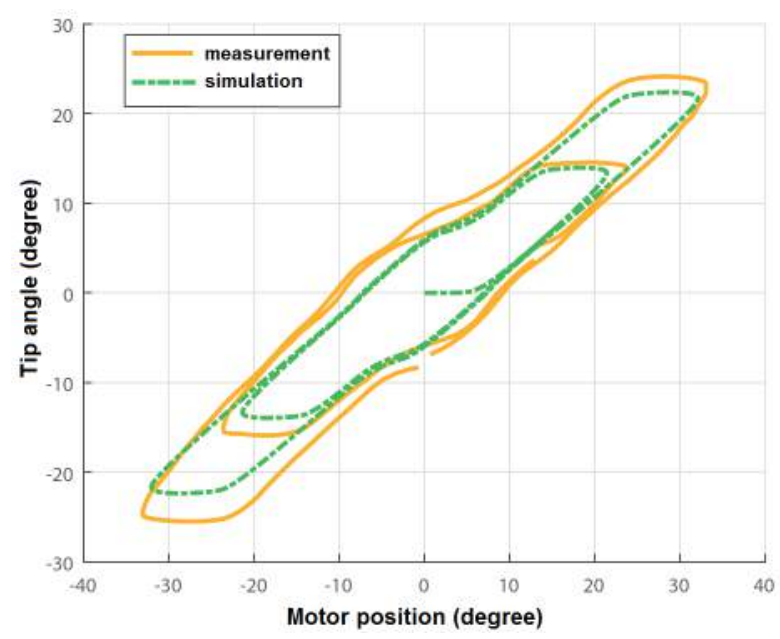

Figure 10.8: Simulation result with one cable bending over one axis. 
A simulation and a measurement were performed. The target was given an offset and the endoscope should be corrected for this offset (Figure 10.9). The 'offset' here is the distance between the target and the center of the image in pixels. The shape of both graphs corresponds, but the size differs. Note that the measurement graph contains plateaus that are missing in the simulation. This is due to the robotic arm stopping before repeating the motion in the measurement setup.
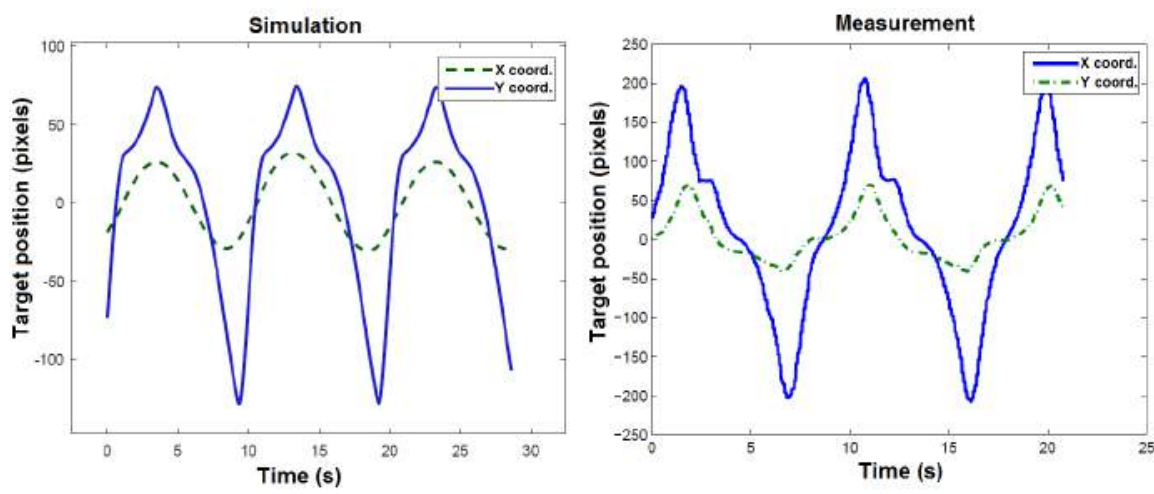

Figure 10.9: The target plate was moved perpendicular to the endoscope tip. The small plateaus in the measurements are due to stopping of the robot arm before initiating a new movement. This was not simulated.

Next, the model was compared to the measurements with a bent tip (Figure 10.10). The solid lines in the figure demonstrate measurement results, once with the tip bent and once with the tip straight. The dashed lines depict simulation results, and these follow the same pattern. Note that in this case an accurately tuned endoscope was used, which is why the middle plateau is almost indiscernible. When the hysteresis compensation control was turned on, the target offset was decreased further than with the control turned off (Figure 10.11).

\section{Latency}

The throughput of the camera stabilization system was between 17 and 22 frames per second (fps). This is well above the earlier stated requirement of a minimum target position refresh rate of 10 times per second. However, from the hysteresis compensation results (Figure 10.11) it becomes clear that latency is present in the control loop. The latency is probably due to buffering in the Olympus video processor. This is deduced from the fact that approximately 7 frames were lost in our setup, and the other data transmissions hubs in the system could not have caused more than a loss of one frame in total. 


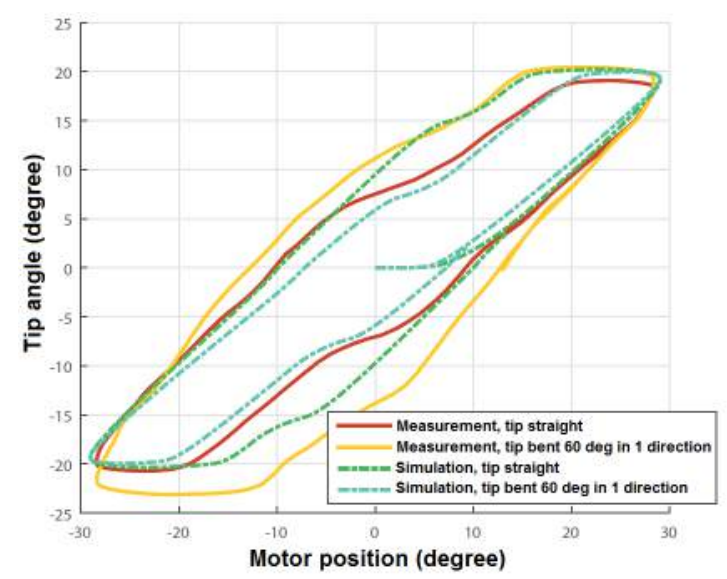

Figure 10.10: Simulation and measurement with straight tip and tip bent in one direction. Observe that the middle plateau shifts and the width of the hysteresis differs.

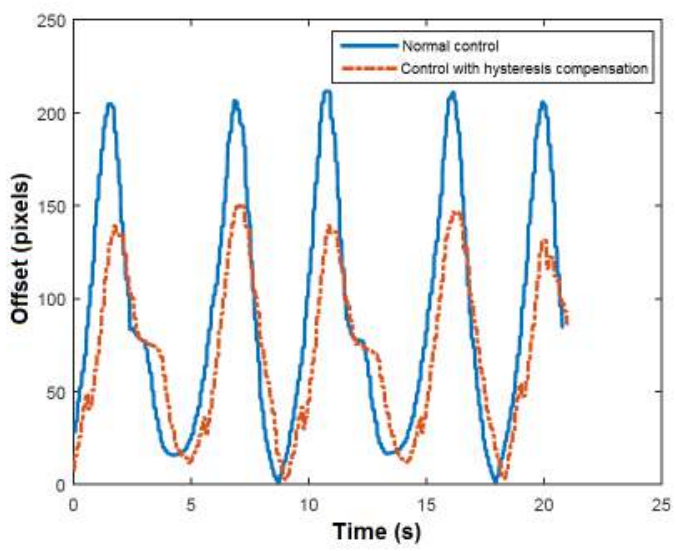

Figure 10.11: The hysteresis compensation was turned on in the red graph. Note that the ultimate offset is smaller.

\subsubsection{Tracking performance}

The produced camera motion is compared to the tracked target in the image (Figures 10.12 and 10.13). The target plate motion was horizontally or vertically perpendicular to the endoscope tip and the speed was gradually increased, but the motion was not actively corrected. From the figures can be seen that drift occurs if the motion becomes too fast, which is to be expected since motion blur will then impair the performance of the tracking algorithm. The camera motion itself is stable and reproducible. 


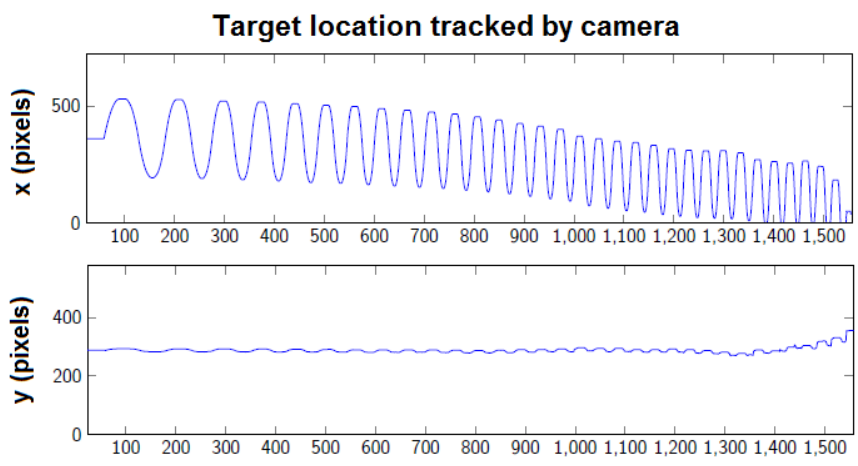

Target location tracked on target plate
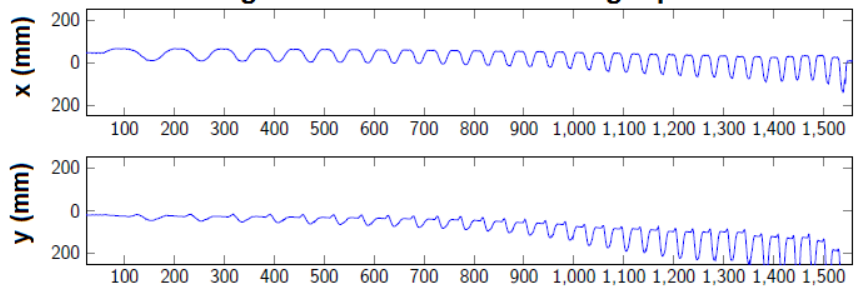

Distance from initialized position

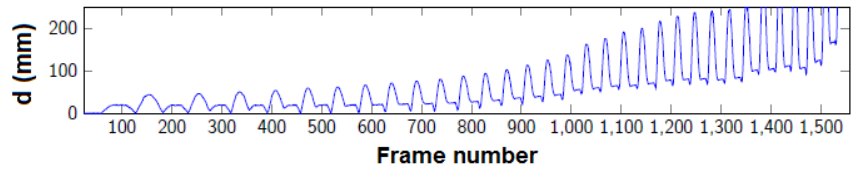

Figure 10.12: The target plate was moved perpendicular to the endoscope tip in a horizontal line. The speed of this motion was gradually increased. The distance from the initial target position should be 0 , because this means that the initial target is re-tracked. However, it can be seen that a drift starts to occur and the distance keeps increasing. Note that the robotic control was not turned on, so the motion is not compensated for.

The tracking algorithm also performed well during the more complex circular motion (Figure 10.14). The peaks are indicating moments where the OptiTrack reflectors were occluded and the and therefore causing a large deviation.

The overall average distance in real world coordinates between the target that was computed through the tracking algorithm, and the target that was considered the ground truth was found to be 25.5( \pm 28.7$) \mathrm{mm}$.

\subsection{Discussion}

In this chapter, a novel approach to a camera stabilization system of flexible endoscopes is presented that is suitable for clinical implementation. The system performed 

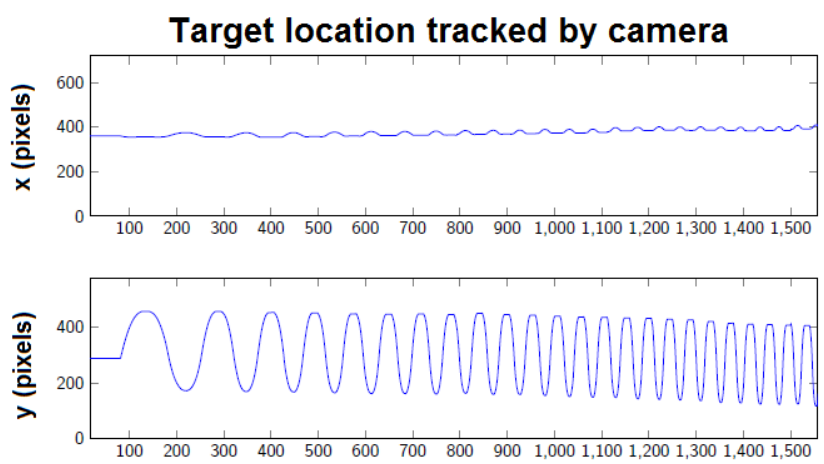

Target location tracked on target plate
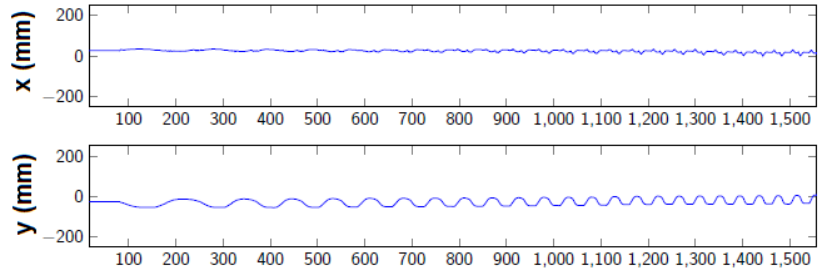

Distance from initialized position

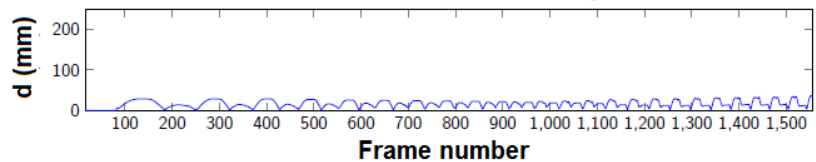

Figure 10.13: The target plate was moved perpendicular to the endoscope tip in a vertical line.

The speed of this motion was gradually increased. Figure similar to Figure 10.12.

in real-time. No true validation measurements were done yet (i.e. solid quantifications and error measures were not used), because some improvements in the system need to be made. These will be discussed in more detail in the rest of this section. This research therefore needs to be regarded as a stepping stone for future research. Nonetheless, from the preliminary evaluation, the system was shown to be feasible and may in the future facilitate complex procedures with conventional flexible endoscopes.

The experimental results for the hysteresis compensation look promising, but also show that improvements in the control and the setup are needed. In general, the system responded as expected, but a remaining offset of 150 pixels is still relatively large (the resolution of the images was $768 \times 576$ pixels). Since the dynamical behavior in the simulations does correspond to the measurement results, we think the current approach to the hysteresis compensation is good, but the parameters for the compensation need to be thoroughly optimized for better system performance.

A latency in the system was present and identified to be approximately 7 frames. 
Target location on board
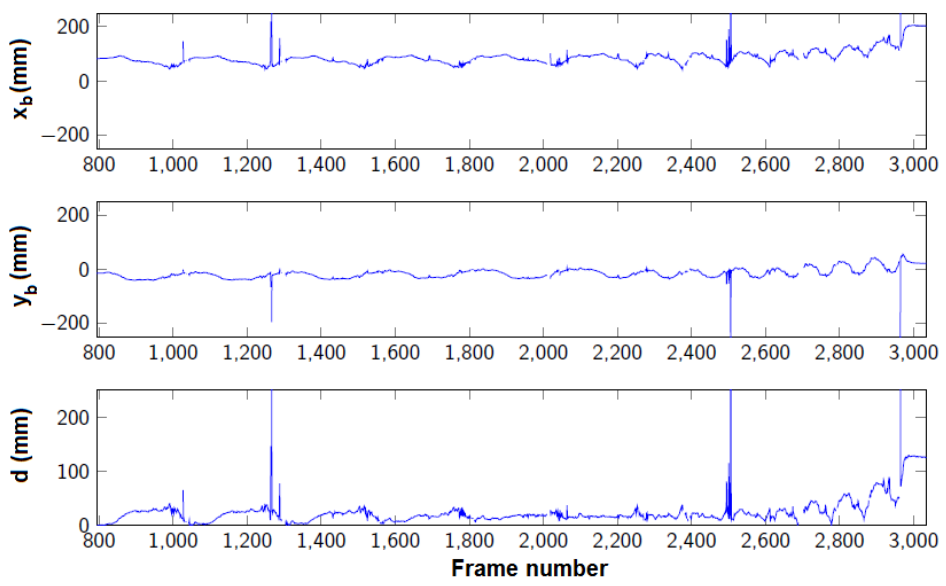

Figure 10.14: The target plate was moved perpendicular to the endoscope tip in a circular motion. The speed of this motion was gradually increased. It can be seen that here, too, there is a threshold in speed after which the target gets lost.

To compensate for system latency, the current implementation makes sure that upon frame acquisition, if a frame is directly available, the next frame is acquired. A directly available frame means that frame was already there, so new information can only be obtained from the next one. If this happened, the next frame was therefore acquired. This implementation caused good system performance. However, although the latency in the system may now have been compensated for, a latency-free system would of course be more desirable. To achieve such a system, some code and implementation optimization may help, but the main cause for latency is likely the currently used video processor.

The tracking results in itself were shown to be robust up to a threshold above which the speed became too high. The exact threshold value depends on the application (camera and environment influence this threshold), and the amount of drift that is acceptable therein. A lack of robustness against high speeds is only solvable if additional sensors (e.g. accelerometers) are added to the endoscope tip for enhanced position and orientation measurements.

In terms of accuracy we measured a mean distance of $25 \mathrm{~mm}$ between the ground truth and the tracked target. This is relatively large, considering the environment the endoscope is normally operated in has a size of a few millimeters. However, we think this inaccuracy may largely be due to the design of the experimental setup, and specifically the scale at which experiments were performed. The endoscope is designed for small motions in a small space. The currently used setup was several measures larger, and accuracy validation should be done in an experiment that resembles the scale during endoscopic therapy better. That said, the currently used setup did provide a good 
insight in system behavior in general. The physical interactions of the system are clarified now, but no reliable quantitative results can be obtained in terms of accuracy.

A last recommendation for future research involves the endoscopic cable adjustment. At the time of the experiments, all the endoscopes that were available were recently tuned by the manufacturer. This means the cable slackness was nullified to improve tip response. Although the upside was that hysteresis was decreased, the downside was that we could not reliably test our compensation mechanism. It is vital to measure this mechanism thoroughly, since hysteresis correction can be dangerous. The hysteresis is compensated for by letting the motors move to the expected end position really fast. However, if the estimation is inaccurate, this fast motion may lead to overshoot and/or patient damage.

\subsection{Conclusion}

The design of the tracking and control of an endoscope stabilization system was described, and the preliminary evaluation provides a basis for future research. Although the system works in real time and is compatible with the current clinical workflow, some issues with the system and the experimental setup need to be overcome. The main recommendations are to use a testing environment that resembles reality better in terms of dimensions and to perform a more thorough parameter optimization for the hysteresis compensation mechanism.

The target tracking was robust, but high-speed target displacements cause motion blur and this in turn causes the tracking algorithm to fail. Although no quantitative conclusions are possible in terms of accuracy, we expect the tracking to perform well during the application we have in mind (complex flexible endoscopic interventions). However, there still is a long way before clinical implementation is feasible.

\subsection{References}

Please refer to the bibliography at the end of this thesis. 
CHAPTER 11

\section{Conclusions}

Malcolm Forbes

This chapter contains the conclusions and final remarks of this dissertation. 


\subsection{Summary of findings and conclusions}

There is no doubt that endoscope control currently is non-ergonomic and non-intuitive. Robotization of the steering is one way to facilitate endoscope control, but imageguidance can help the endoscopist do what he currently can not. The main objective of the described work was to develop and validate image-based methods for automated flexible endoscope navigation. This currently is an active field of research, which means others have tried to achieve comparable goals. The main advantage of the approach presented here over other existing approaches was the continuous focus on clinical relevance. The findings and the conclusions drawn from them are summarized here, and recommendations for future work are provided.

This thesis can roughly be divided in two main subjects: automated navigation during endoscope diagnostics and endoscope stabilization during interventions. The conclusions will therefore be presented separately.

\subsubsection{Flexible endoscope navigation automation}

The main goal of this part of the thesis was to develop automated methods for easy endoscope introduction. By controlling the tip steering of the endoscope, it is expected that learning to perform an endoscopy will become easier. In automated control, a correctional steering motion is made each time the current heading direction deviates from the target direction. The direction of the endoscope can be obtained by using the images the endoscope makes. Determining camera motion means determining endoscope motion: after all, the endoscope camera is located at the tip. Estimating camera motion was done before using the projected object displacements in consecutive images which can be obtained from the optical flow. This approach was now also used in endoscopy, as described in Chapter 3. The projected object displacements may be derivable from a pixel displacement field, but a model is needed to translate the found projected displacement to an actual 3D motion. The Focus of Expansion is shown in Chapter 3 to be a useful navigational clue for optical flow interpretation from endoscopic images. The manually indicated heading direction in image sequences corresponded well with the automated results that were obtained with the novel motion estimation model.

However, the Focus of Expansion model only provides accurate information under the assumption of translational motion. Since rotational motion is present, too, a motion reconstruction can best be obtained from the complete epipolar geometry that can be obtained from the optical flow field between images. As shown in Chapter 5, it is theoretically possible to reconstruct the rotation and translation of the camera under certain conditions. One of the conditions is that enough optical flow displacement vectors need to be present. Endoscopic images often lack texture and suffer from artifacts, two properties that complicate optical flow estimation. The results therefore depend heavily on the method of optical flow estimation. A selection of different methods that were deemed suitable for our application, resulting in both dense and sparse flow fields, was therefore implemented and evaluated in Chapter 4. From this evaluation, the Lucas-Kanade dense optical flow was chosen to be the best method in the 
case of flexible endoscopy. Moreover, a novel methodology for systematic optical flow performance comparison was designed for this evaluation. This methodology is, as described in Chapter 4 as well, easy to implement and can be used for every application that requires optical flow. However, if a region-based displacement tracking method is employed, the fundamental matrix estimation will differ, which means the methodology needs to be adjusted accordingly. The overall motion estimation approach that was presented in Chapter 5 is currently deemed too unreliable for an application in flexible endoscopy navigation (see also the recommendations on this subject).

Assuming that the right optical flow method can be found and a suitable motion model is chosen, it is possible to estimate endoscopic heading direction from endoscopic images. This heading direction needs to be compared to the desired or target direction. The deepest or darkest area in endoscopic images was investigated before for its use in automated endoscope navigation. However, the approach always was fully autonomous navigation by centralizing the target region. When an endoscopist performs an endoscopy, the endoscope is often inserted based on the known heading direction or based on pre-procedural knowledge. The endoscope tip therefore should only be corrected if the current heading direction of the endoscope deviates from the target direction. With this objective, we validated and implemented a novel and realtime lumen detection algorithm (Chapter 6). The heading direction information was not obtained automatically, but by the endoscopist himself. This way, accuracy of the heading direction estimation was ensured. The estimated target direction was displayed in the endoscopic view through a small white circle. If the endoscopist then wanted to correct the current heading direction towards the estimated target direction, this could be achieved by means of a button press. The feasibility of this navigation approach was now determined in two ways:

1. Expert and novice users were asked to perform a simulated colonoscopy with and without the system and filled out a questionnaire (Chapter 7)

2. During the colonoscopies the procedure times and phases were registered, as well as the use of the automated functionality (Chapter 8)

Especially novice users were enthusiastic about the system. They found the automated colonoscopy to be easier and more intuitive. Expert users saw clinical potential for the system. They did not find the new system easier, but could imagine that it would be easier to learn colonoscopy with this system for novice users. Some improvements in system functionality and experimental setup were suggested as well. The feedback screen that was now used to provide information about tip flexion/bending was unclear for some people. The system was designed for an improved endoscope insertion, but experts saw additional value during colonic inspection, so when the endoscope is retracted. Overall use of the system however was limited. The navigational guidance needed to be turned on actively by keeping a button pressed, and this only was done during a few per cent of the time in the procedure. The main reason for this probably was the limited time that participants received to practice with the system. Because this was a feasibility pilot, the choice was made to grant the participants only a familiarization period right before the experiment. In hindsight, this period probably was too limited, and possibly multiple training sessions would have improved the use of the 
navigation guidance. However, one of the study objectives was to assess intuitiveness of the system, and this could not have been done with fully trained participants.

\subsubsection{Flexible endoscope stabilization}

The second part of this thesis was more focused on endoscope usage during interventions. Because of the limited dexterity an endoscopist has during interventions, mostly caused by the long and flexible instruments with limited degrees of freedom, technological assistance may improve endoscopic interventions. An observation was that during endoscopic interventions, at least one person was needed to fixate the endoscope and another person to operate the endoscopic instrument(s).

With the robotized endoscopy system, it was theoretically possible to fixate the endoscope robotically. It would be better however, to fixate the endoscope on a region of interest that the endoscopist can indicate at any point during the procedure. This way, camera control still is a task of the endoscopist but is also flexible enough for the continuously changing environment that these procedures take place in. The software that was developed earlier and described in Chapters 3-5 can be employed to realize exactly such a funtionality. The general idea is to not only measure camera motion with respect to the environment, but correct for it at the same time. The main requirements for such a system then became accurate motion tracking and real-time performance. Between these two features unfortunately a trade-off exists. Nonetheless, a suitable algorithm was developed, as described in Chapter 9 . This algorithm was robust against artifacts caused by smoke, fluids or instruments in the field of view. The developed algorithm does have some difficulty handling large motions.

Experimental validation of the implemented algorithm using the Teleflex system showed that the tracking algorithm performed accurately. The fast motion robustness issue was taken into account in the implementation of the robotic control. The experiments provided a proof of concept for the system, but especially in the robotic control many things need to be improved before it is suitable for clinical practice.

\subsubsection{Overall conclusion}

In conclusion, we can state that image-based endoscope navigation is not only feasible, it also shows potential for extended system functionality during diagnostics as well as interventions. Novices indicate that a robotized system with automated navigation is easier to use than the conventional endoscope system. Examples of extended functionality include the navigation guidance during retraction in diagnostic endoscopy, and the camera stabilization during interventional endoscopy. Naturally, the software applications that were developed during the course of this research require a robotic endoscopy system to be present first. Nonetheless, the future for safe, efficient and complex endoscopies using an image-guided, robotized system looks promising. 


\subsection{Recommendations}

All in all, one of the conclusions after four years of research still is that endoscopy will ultimately benefit from robotization and/or image guidance. A recommendation for endoscopists therefore is to be open to new systems and methods. A recommendation for engineers or endoscope producers is to provide solutions that endoscopists find good enough.

More specific recommendations that can be directly derived from this thesis are categorized in the two parts of this thesis and are discussed accordingly below. Some recommendations are subject-independent and are provided in the last section of this chapter.

\subsubsection{Flexible endoscope navigation automation}

Although major steps were taken already, as described in this thesis, automated flexible endoscope navigation can be improved. Finding the current heading direction of the endoscope proved difficult. It is recommended to find a better motion estimation model, possibly with the use of artificial neural network learning, as Bell et al. tried [85]. Key is here to find a model that is more reliable with 'difficult' images to diminish the number of highly deviating motions. Therefore, the testing environment need to be given careful thought. Real tissue images differ significantly from plastic model images. In addition, the ultimate clinical application and workflow need to be leading for choices in system development. If a system is going to work, it needs to fulfill certain requirements (Chapter 5), and developmental choices need to be made accordingly.

A problem with the used model for camera motion estimation in this thesis was the large variance that existed in the estimation. After all, the motion data will be used as robotic control input and large motion variance of the robot is not desired. A better motion estimation model might solve this problem, but another approach could be to filter the estimated outcome. If the input for the robotic control is smoothened beforehand, good results may be achieved as well, and this approach will probably take less time.

Finding the target direction can be done reliably already, as described in chapters 6 through 8 . However, to truly evaluate an assisting navigation algorithm among endusers, a more elaborate study will be necessary. Not only a larger participant group needs to be used then, but participants have also proven to need more time for system familiarization. Since system familiarity influences the results substantively, it is highly recommended to pay close attention to this detail when setting up the study.

\subsubsection{Flexible endoscope stabilization}

The idea of flexible endoscope stabilization is an appealing one, but much more work will have to be done on this subject. From Chapter 9, the major recommendation was to improve algorithm robustness against large motions. A work-around for this problem was demonstrated in Chapter 10 by ignoring the unreliable data. Ideally of course, a more robust, but also a faster algorithm is desired. However, code optimization and hardware programming may be of enough help here already. The stabilization system 
as it was currently presented must be regarded as a proof of concept.

The prototype as employed in Chapter 10 is useful for in vitro evaluation of the system, but for clinical evaluation other steps need to be taken as well. In the limited setup that was available, only recently serviced endoscopes were tested. However, the hysteresis and deadband vary greatly between endoscopes, hospitals and points in time. Therefore, it is recommended to test a large range of endoscopes, from different hospitals, too, before concluding on system functioning.

Next to the choice of endoscope, the choice of testing environment needs to be carefully made. The results in Chapter 4 show that this type of image-based navigation is highly application-specific. The test environment therefore needs to resemble the clinical context as much as possible. This means not only the images need to be representative, but also the people using the endoscopes and the maneuvers they make with them. The ultimate test would be in an in vivo environment, but ethical issues need to be considered well and a thorough risk analysis needs to be carried out. However, before starting true clinical evaluation, an investigation into the possible clinical applications is recommended. Adding a robotic system to endoscopic procedures will only be beneficial if:

1. The procedure already is time-consuming

2. The procedure currently has other unacceptable consequences

3. The procedure can not be performed at all due to technological limitations

Identifying suitable procedures is key to developing this technology into a clinical success.

\subsubsection{General recommendations}

This thesis is an example of applied technological research with a continuous focus on in this case clinical relevance. In the course of the research presented here, much feedback was gathered on the system, but also on the approach towards developing it. The majority of opinions on this approach was very positive. The main recommendation for future research is therefore to always and continuously involve multiple end-users in the development of new technologies. The new technology always needs to improve the current practice and the only way to ensure it will is asking the current practitioners. Spending time with end-users prevents researchers from wandering off in a direction that may be technologically interesting, but is practically useless. Moreover, it provides inspiration for new projects and in the end, researchers are always seeking inspiration. 


\section{Camera calibration using magnetic tracking}

This appendix contains a camera calibration method for any camera to which an electromagnetic tracking sensor is attached. This work has been compiled over the years and can be used in many applications. It forms the basis for the analyses done with endoscopic cameras in chapters 5 and 10 of this thesis. 


\section{Introduction}

A generic calibration procedure for a camera is described, with an example using a webcam and a magnetic tracking system. The aim of a general camera calibration is mostly to establish the geometrical and optical properties of the camera with respect to the image and/or the world.

First, the data acquisition process will be explained with the aim to make it easily reproducible. Second, an example is provided which can be used as the basis for data analysis. The example shows a possible experimental validation setup for visual motion estimation. The described approach can be applied to any setup in which a camera and another position measurement system (e.g. OptiTrack) are present.

\section{Part I: Data acquisition}

The camera calibration procedure is described below in a manual-like manner. For an elaborate yet understandable theoretical background on the subject, we would like to refer the reader to [96].

\section{Experimental setup}

The experimental setup is designed for a validation experiment of motion estimation algorithms. This validation is established by first conducting a generic camera calibration, and then estimating camera motion from the images. A second position measurement system is employed to validate the outcome of the motion estimation algorithm. In this case, the second modality is the electromagnetic NDI Aurora tracking system.

\section{Materials}

- NDI Aurora V2:

- Field Generator Table Top

- System Control Unit

- Sensor Interface Unit

- NDI Sensors:

* 6D reference sensor

* 6D pointer tool

- Cables (USB/power)

- Camera (in this case: Philips Webcam SPC900nc/00 with PixelPlus)

- Computer (in this case: a HP Elitebook 8560w) with:

- NDI toolbox

- MATLAB

* Camera calibration toolbox (pre-installed after Matlab 2013b)

- Optionally: driver to read images from camera

- Perspex rod for camera fixation and control 
- Tape for sensor fixation

- Table (without metal frame)

- Checkerboard with marked origin

\section{Methods}

Data acquisition is done using the $\mathrm{m}$-file 'calib_acquire. $\mathrm{m}$ ', designed for MATLAB. Some sub-functions are needed for the script to work properly, specifically the 'ndi_ $\langle\ldots\rangle$. m' functions. All software can be obtained by contacting the author.

The system should be setup as follows. Put the NDI Field Generator on a table and connect the necessary cables according to the manual. Put the checker board on top of the Generator, but make sure the board is within the measurement field (optionally, use the included NDI Track software for this). If not done yet, connect the laptop and the camera. Make sure the images can be read and recorded. Sometimes it is necessary to turn off the built-in webcam. Also check the sharpness of the image, and adjust the camera (lens) if necessary. The experimental configuration of the different parts of the system and their respective coordinate frames is indicated in Figure A.1.

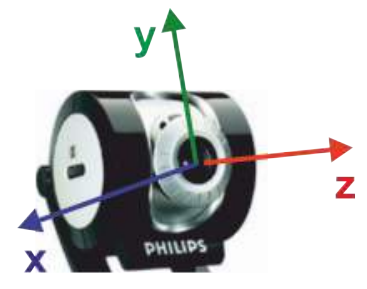

camera

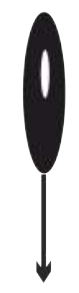

pointer

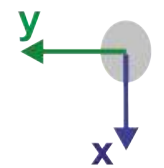

reference sensor

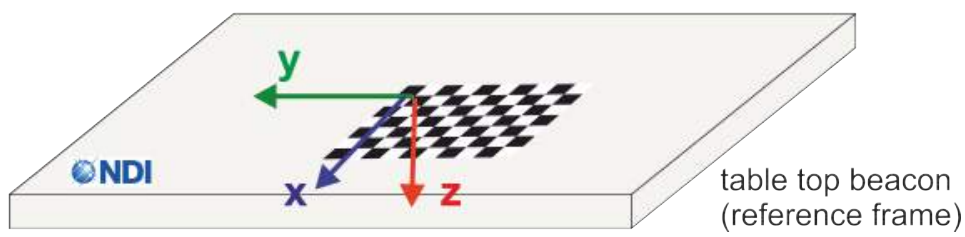

Figure A.1: Configuration of the calibration setup with the different coordinate frames and their orientations.

The calibration data acquisition consists of two parts: obtaining checker board images with a camera equipped with an electromagnetic (EM) tracking sensor, and obtaining the exact corner point position of the checker board using a magnetically tracked pointer.

NB: The positioning data output from the NDI system is given in Euler angles. Spin$\mathrm{Cal}$. $\mathrm{m}$ can be used to transform data from any geometrical description (Euler angles, quaternions, rotation matrices) to any other. However, pay close attention to the order in which the Euler angles need to be defined. The Euler Angle configuration of the NDI 
system is following the ' $Z Y X$ ' standard. If rotation matrices are chosen to represent orientation data, pay attention to the multiplication definition. In SpinCalc.m, premultiplication of a vector with the rotation matrix is assumed. For the data acquisition in this manual, this means that the rotation matrices resulting from SpinCalc.m need to be transposed (=inverted).

Camera data acquisition First, attach a magnetic tracking sensor to the camera in the desired orientation. In this case a reference sensor was used, with the Z-axis of the sensor in the direction of the optical axis of the camera. Check whether the camera sensor is still in range of the field generator. Do not move the checker board during these measurements!

Make sure all the inputs of the m-file match the used checker board dimensions, and that the right amount of calibration images will be taken. The number of necessary images depends on image quality, but should be between 20 and 25. Now, start taking the checker board pictures with the camera, as shown in Figure A.2.

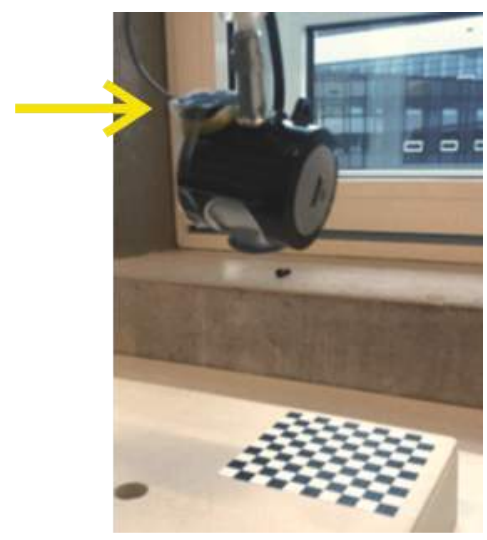

Figure A.2: Camera position during acquisition of checker board calibration data. Attached EM sensor indicated by arrow.

The acquisition script will show the resulting images and ask if they are satisfactory. If ' $\mathrm{n}$ ' + Enter' is typed, the image is ignored and a new image can be made. Otherwise, the image, the position data $(m P(:, i))$ and the orientation data $(m Q(:, i))$ are stored in the indicated folder sdir. The script pauses after $n$ acceptable images are recorded. The corner point locations can now be indicated. Again, do not move the checker board!

Corner point location acquisition Switch the NDI input connector from the reference sensor connector to the pointer sensor connector. This should not give any errors if you do not turn anything else off. Now, press any key and indicate the corners of the checker board. Follow the pattern as shown in Figure A.3.

For the most accurate result, do not change the orientation of the pointer. The 


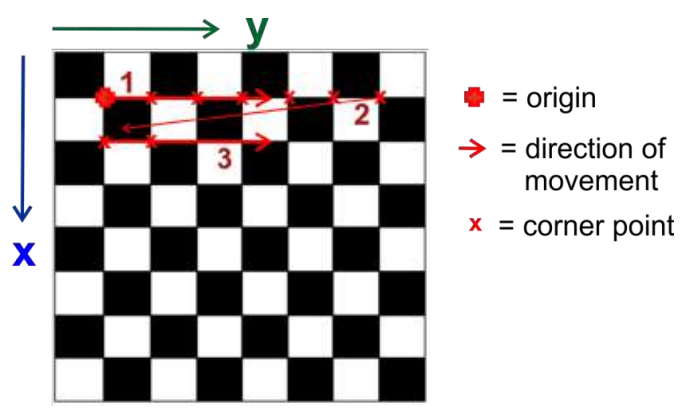

Figure A.3: Pattern of corner point indication during acquisition. Note that the origin is equal to $\left[\begin{array}{ll}X & Y\end{array}\right]=\left[\begin{array}{ll}0 & 0\end{array}\right]$.

calibration gives the best results if the pointer is held in the orientation of Figure A.1. After all the corners are indicated the resulting pattern will be shown. The calibration data acquisition is now finished.

\section{Part II: Data analysis}

Two types of camera parameters can be extracted: intrinsic and extrinsic parameters. Intrinsic camera parameters are independent of camera motion and fixed, such as the focal length and the lens distortion. Extrinsic parameters are obtained using the known checker board dimensions describe the camera pose in 3D world coordinates. Camera calibration needs to be performed only once before a camera is used for measurements assuming no lens repositioning is done.

\section{Intrinsic parameters}

The intrinsic camera parameters are obtained by the camera calibration toolbox. From Matlab 2013 onward it is built-in in the Computer Vision Toolbox, but for other Matlab users it can be downloaded for free online [105]. A full manual of how to use this toolbox is provided online as well. The only required input for the toolbox are the produced checkerboard images and the number and dimensions (in millimeters) of the checkerboard squares. This is all explained in detail on the website. Output is a calibration data set of which the most relevant part is the calibration matrix ' $\mathrm{KK}$ '.

The intrinsic parameter camera matrix or calibration matrix $K$, can be defined as follows:

$$
K=\left[\begin{array}{ccc}
f s_{x} & s_{\theta} & o_{x} \\
0 & f s_{y} & o_{y} \\
0 & 0 & 1
\end{array}\right]
$$

Here, $f$ stands for the focal length, $s_{x}$ or $s_{y}$ for the pixel size in the $x$ - or $y$ direction and $o_{x}$ or $o_{y}$ are the $x$ - and $y$-coordinates of the principal point in the image reference frame. 


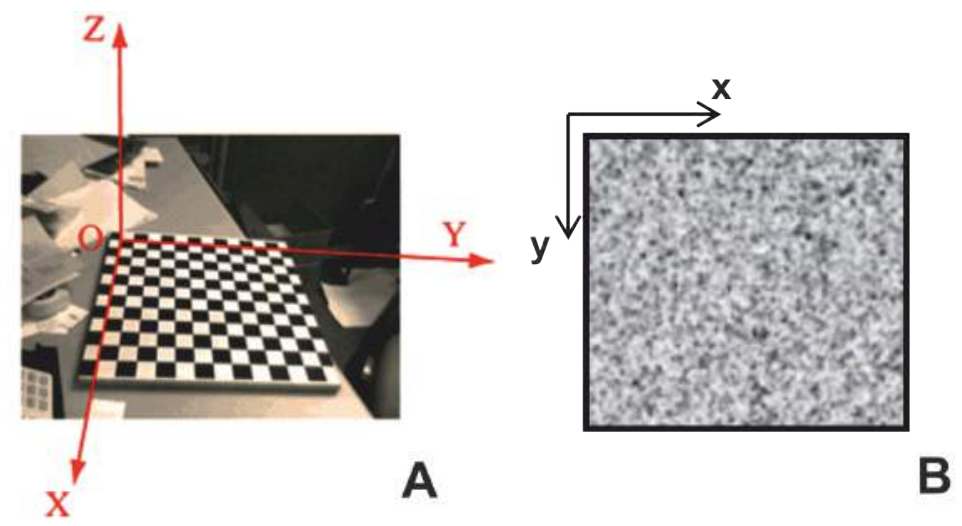

Figure A.4: [A]: Frame of checker board in image. [B]: Origin and coordinates of pixel positions [105].

\section{Extrinsic parameters}

Extrinsic camera parameters can be used to obtain camera motion from the images. From the previous steps we obtained the intrinsic camera parameters and the calibration matrix $K$. Now, we want to express the points that are projected in the image $p$, currently in pixel coordinates, in the camera reference frame $\mathbf{c}$ in metrics. In [96], the relation between pixels $\mathbf{x}^{\prime}$ and $3 D$ coordinates $\mathbf{X}$ is stated as:

$$
\lambda \mathbf{x}^{\prime}=K \Pi_{0} \mathbf{X}=K \Pi_{0} g \mathbf{X}_{0} \text { with } \Pi_{0}=\left[\begin{array}{lll}
1 & 0 & 0 \\
0 & 1 & 0 \\
0 & 0 & 1
\end{array}\right]
$$

Here, $\lambda$ is a scale factor, which under the linear perspective projection assumption equals the depth between $\mathbf{x}^{\prime}$ and $\mathbf{X}$ ( $z$-direction of the camera frame in this case). $K$ is the camera calibration matrix and $g$ the rigid body transformation from the world frame to the camera frame. $\Pi_{0}$ represents the standard projection matrix. It usually is the challenge here to find transformation $g$. This transformation can be estimated using the knowledge about the checker board dimensions and the intrinsic camera parameters. However, using the Camera Calibration Toolbox a simple press on the 'Extrinsic' button will do the work for you. The transformation matrices for all camera positions are generated automatically, and a figure similar to Figure A. 5 should appear. 


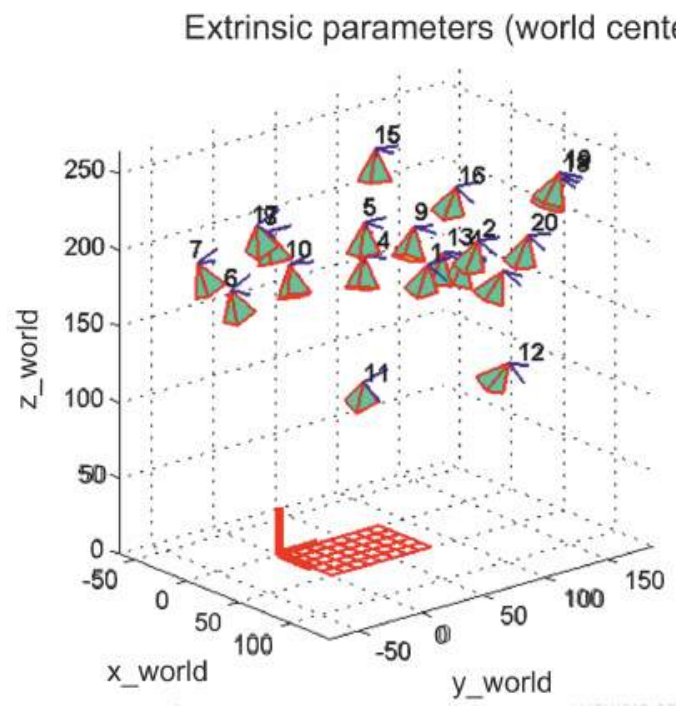

Figure A.5: Extrinsic results from the camera calibration toolbox.

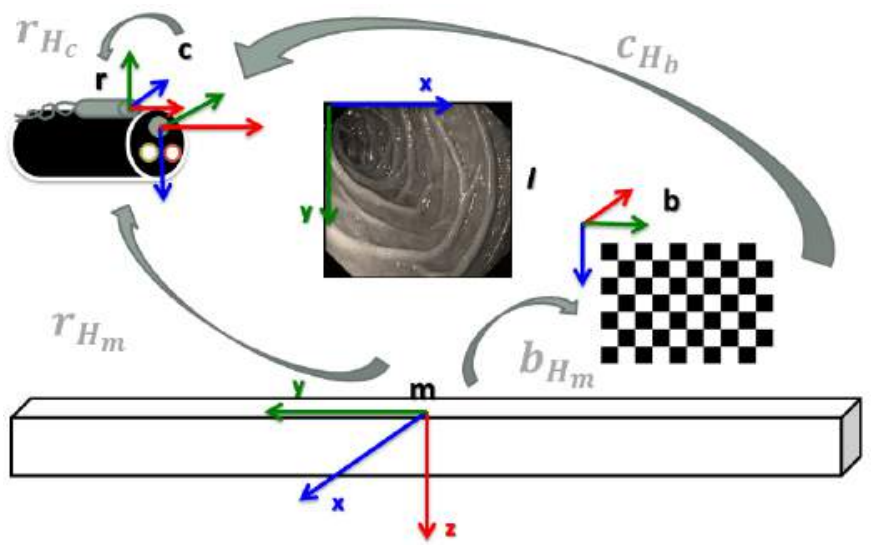

Figure A.6: Transformation matrices, denoted by $H$, as defined during the calibration.

\section{Example: compute other transformations}

The aim of this subsection is to define the transformation matrix between the camera and the reference sensor, ${ }^{{ }^{r}} \mathbf{H}_{\mathbf{c}}$ (Figures A.6 and A.7). Knowledge of this transformation is needed to use the EM tracking results for camera motion estimation vali- 


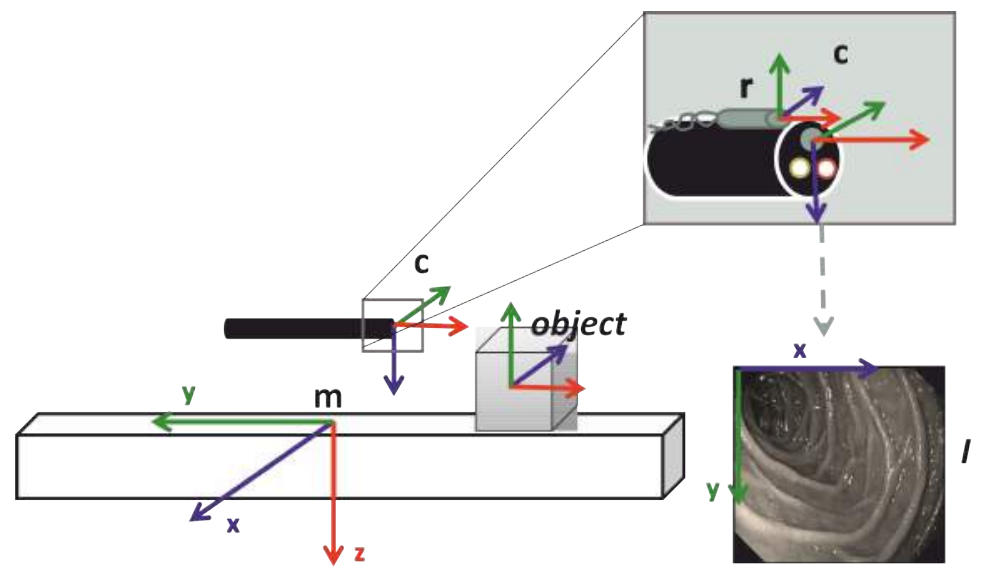

Figure A.7: Coordinate frames as defined in the example validation setup.

dation. The computation is based on the underlying assumption that the position of the checker board is fixed and known using two measuring modalities: the camera (through the 'Extrinsic' functionality), and the EM tracking system (through the sensor and magnetic beacon measurements). The transformation between the sensor and the camera is then needed to express the reference sensor data in the camera coordinate frame.

${ }^{\mathrm{r}} \mathbf{H}_{\mathbf{c}}$ can be computed as follows. Let ${ }^{\mathrm{b}} \mathbf{s}_{\mathbf{m}}$ be the corner points of checker board $\mathbf{b}$, expressed in coordinate frame $\mathbf{b}$, measured with respect to magnetic beacon $\mathbf{m}$. Assuming a rigid (body) transformation, the $4 \times 4$ homogeneous transformation matrix ${ }^{\mathrm{b}} \mathbf{H}_{\mathrm{m}}$ can be used to describe the displacement of all points within the frame:

$$
{ }^{\mathrm{b}} \mathbf{H}_{\mathrm{m}}=\left[\begin{array}{cc}
{ }^{\mathrm{b}} \mathbf{R}_{\mathrm{m}} & { }^{\mathrm{b}} \mathbf{t}_{\mathrm{M}} \\
\mathbf{0}_{3} & 1
\end{array}\right]
$$

in which ${ }^{\mathbf{b}} \mathbf{R}_{\mathbf{m}}$ is the $3 \times 3$ rotation matrix between the origins of frames $\mathbf{b}$ and $\mathbf{m}$, and ${ }^{b} t_{m}$ the $3 \times 1$ translation vector. The corner point positions in $\mathbf{m}$ are the ones obtained using the NDI pointer tool. The position of the camera is recorded with the reference sensor (frame $\mathbf{r}$ ). $\mathbf{R}_{\mathbf{c}}$ and $t_{c}$ result from the Camera Calibration Toolbox and represent the rotation matrix and translation vector of $\mathbf{c}$ with respect to $\mathbf{b}$. These can be used to compute ${ }^{\mathbf{c}} \mathbf{H}_{\mathbf{b}}$ in a similar fashion as shown in eq. A.3. The transformation matrix between $\mathbf{m}$ and $\mathbf{r}$ is obtained from the measured camera positions by $\mathbf{r}$. Ultimately, the transformation matrix between the sensor frame $\mathbf{r}$, expressed in camera coordinates (c) is computed from:

$$
{ }^{\mathrm{c}} \mathrm{H}_{\mathrm{r}}={ }^{\mathrm{c}} \mathbf{H}_{\mathrm{b}}^{\mathrm{b}} \mathbf{H}_{\mathrm{m}}^{\mathrm{m}} \mathbf{H}_{\mathrm{r}}
$$

To check whether the calibration was successful and accurate, the camera position obtained from the Camera Calibration Toolbox can be compared to the camera 
position that can be computed using the EM data. The first can be obtained directly from $R_{-} c$ and $T_{-} c$. The latter can be obtained from the following homogeneous transformation matrix multiplication:

$$
{ }^{\mathrm{c}} \mathrm{H}_{\mathrm{b}}={ }^{\mathrm{c}} \mathbf{H}_{\mathrm{r}}^{\mathrm{r}} \mathrm{H}_{\mathrm{m}}^{\mathrm{m}} \mathrm{H}_{\mathrm{b}}
$$

Note that the board is now expressed in the camera frame, but this will result in a better

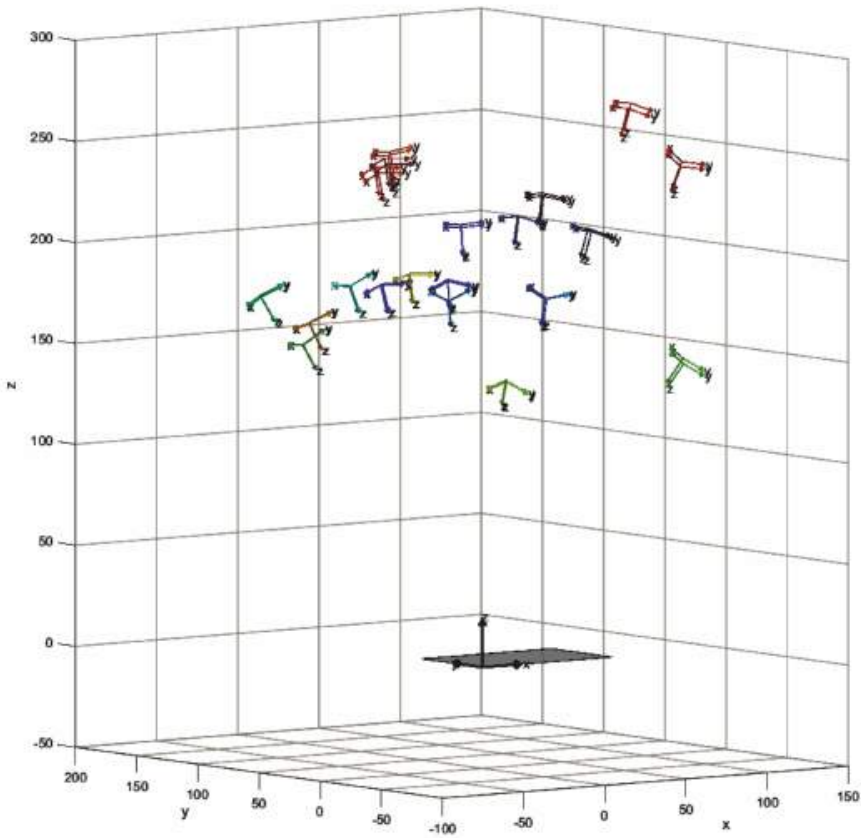

Figure A.8: Results of comparison of the camera positions, obtained with both position measurement systems.

visualization of the results (Figure A.8). To obtain this figure, 'ut_plot_axis3D .m' can be employed.

\section{Validate camera motion estimation}

For the complete example of the ultimate validation analyses, please refer to Chapter 5.

\section{A.1 References}

Please refer to the Bibliography at the end of this thesis. 


\section{Bibliography}

[1] M. Reza, J. Blasco, E. Andradas, R. Cantero, and J. Mayol, "Systematic review of laparoscopic versus open surgery for colorectal cancer," British Journal of Surgery, vol. 93, no. 8, pp. 921-928, 2006.

[2] G. H. Ballantyne, "Robotic surgery, telerobotic surgery, telepresence, and telementoring. Review of early clinical results." Surgical endoscopy, vol. 16, no. 10, pp. 1389-402, Oct. 2002. [Online]. Available: http://www.ncbi.nlm.nih.gov/pubmed/12140630

[3] L. Zamorano, Q. Li, S. Jain, and G. Kaur, "Robotics in neurosurgery:state of the art and future technological challenges," International Journal of Medical Robotics and Computer Assisted Surgery, vol. 01, no. 01, p. 7, 2004. [Online]. Available: http://www.roboticpublications.com/index.php?page=abstract0101\\&id=2

[4] M. H. Whiteford and L. L. Swanstrom, "Emerging Technologies Including Robotics and Natural Orifice Transluminal Endoscopic Surgery ( NOTES ) Colorectal Surgery," Journal of surgical oncology, vol. 96, no. September, pp. 678-683, 2007.

[5] M. Chmarra, W. Kolkman, F. Jansen, C. Grimbergen, and J. Dankelman, "The influence of experience and camera holding on laparoscopic instrument movements measured with the TrEndo tracking system." Surgical endoscopy, vol. 21, no. 11, pp. 2069-75, Nov. 2007. [Online]. Available: http://www.ncbi.nlm.nih.gov/pubmed/17479335

[6] R. Van Der Schatte Olivier, C. Van't Hullenaar, J. Ruurda, and I. Broeders, "Ergonomics, user comfort, and performance in standard and robot-assisted laparoscopic surgery," Surgical Endoscopy and Other Interventional Techniques, vol. 23, no. 6, pp. 1365-1371, 2009.

[7] K. Cleary and T. M. Peters, "Image-Guided Interventions: Technology Review and Clinical Applications," Annual Review of Biomedical Engineering, vol. 12, no. 1, pp. 119-142, 2010. [Online]. Available: http://www.annualreviews.org/doi/abs/10.1146/ annurev-bioeng-070909-105249

[8] M. Baumhauer, M. Feuerstein, H.-P. Meinzer, and J. Rassweiler, "Navigation in endoscopic soft tissue surgery: perspectives and limitations." Journal of endourology / Endourological Society, vol. 22, no. 4, pp. 751-66, Apr. 2008. [Online]. Available: http://www.ncbi.nlm.nih.gov/pubmed/18366319

[9] R. Reilink, "Image-based robotic steering of advanced flexible endoscopes and instruments," Doctoral degree, University of Twente, 2013. [Online]. Available: http://purl.org/utwente/doi/10.3990/1.9789036535267

[10] V. Karimyan, M. Sodergren, J. Clark, G.-Z. Yang, and A. Darzi, "Navigation systems and platforms in natural orifice translumenal endoscopic surgery (NOTES)." International journal of surgery (London, England), vol. 7, no. 4, pp. 297-304, Aug. 2009. [Online]. Available: http://www.ncbi.nlm.nih.gov/pubmed/19481186

[11] B. P. M. Yeung and T. Gourlay, "A technical review of flexible endoscopic multitasking platforms." International journal of surgery, vol. 10, no. 7, pp. 1-10, May 2012. [Online]. Available: http://www.ncbi.nlm.nih.gov/pubmed/22641123 
[12] E. I. Schippers, "Besluit invoering bevolkingsonderzoek naar darmkanker," Ministerie van Volksgezondheid, Welzijn en Sport, Tech. Rep. 261, 2011.

[13] H. van Veldhuizen-Eshuis, M. Carpay, J. van Delden, L. Grievink, B. Hoebee, A. Lock, and R. Reij, "Uitvoeringstoets bevolkingsonderzoek naar darmkanker," RIVM, Den Haag, Tech. Rep., 2011.

[14] L. C. Seeff, T. B. Richards, J. A. Shapiro, M. R. Nadel, D. L. Manninen, L. S. Given, F. B. Dong, L. D. Winges, and M. T. McKenna, "How many endoscopies are performed for colorectal cancer screening? Results from CDC's survey of endoscopic capacity," Gastroenterology, vol. 127, no. 6, pp. 1670-1677, 2004.

[15] A. Menciassi, P. Valdastri, K. Harada, and P. Dario, "Single and multiple robotic capsules for endoluminal diagnosis and surgery," Surgical Robotics: Systems Applications and Visions, pp. 313-354, 2011.

[16] P. Valdastri, M. Simi, and R. J. Webster III, "Advanced technologies for gastrointestinal endoscopy," Annual reviews on biomedical engineering, vol. 14, pp. 397-429, Jan. 2012. [Online]. Available: http://www.ncbi.nlm.nih.gov/pubmed/22655598

[17] S.-H. Lee, I.-K. Chung, S.-J. Kim, J.-O. Kim, B.-M. Ko, Y. Hwangbo, W. H. Kim, D. H. Park, S. K. Lee, C. H. Park, I.-H. Baek, D. I. Park, S.-J. Park, J.-S. Ji, B.-I. Jang, Y.-T. Jeen, J. E. Shin, J.-S. Byeon, C.-S. Eun, and D. S. Han, "An adequate level of training for technical competence in screening and diagnostic colonoscopy: a prospective multicenter evaluation of the learning curve," Gastrointestinal Endoscopy, vol. 67, no. 4, pp. 683-689, 2008. [Online]. Available: http://www.sciencedirect.com/science/article/pii/S0016510707028623

[18] J. J. Vargo, "North of 100 and south of 500: where does the "sweet spot" of colonoscopic competence lie?" Gastrointestinal endoscopy, vol. 71, no. 2, pp. 325-6, Feb. 2010. [Online]. Available: http://www.ncbi.nlm.nih.gov/pubmed/20152312

[19] J. D. Waye, D. K. Rex, and C. B. Williams, Colonoscopy: Principles and Practice, 2nd ed., J. D. Waye, D. K. Rex, and C. B. Williams, Eds. Chichester: Blackwell Publishing Ltd, Aug. 2009.

[20] K. Schwab and S. Singh, "An introduction to flexible endoscopy," Surgery (Oxford), vol. 29, no. 2, pp. 80-84, Feb. 2011. [Online]. Available: http://linkinghub.elsevier.com/retrieve/pii/ S0263931910002498

[21] O. Nahlieli, A. Neder, and A. M. Baruchin, "Salivary gland endoscopy: a new technique for diagnosis and treatment of sialolithiasis," Journal of Oral and Maxillofacial Surgery, vol. 52, no. 12, pp. 1240-1242, May 1994. [Online]. Available: http://www.ncbi.nlm.nih.gov/ pubmed/9223796

[22] T. Fukushima, B. Ishiijima, K. Hirakawa, N. Nakamura, and K. Sano, "Ventriculofiberscope: a new technique for endoscopic diagnosis and operation," Journal of Neurosurgery, vol. 38, no. 2, pp. 251-256, 1973.

[23] M. Pedrosa, F. Farraye, A. Shergill, S. Banerjee, D. Desilets, D. Diehl, V. Kaul, R. Kwon, P. Mamula, S. Rodriguez, S. Varadarajulu, L.-M. Song, and W. Tierney, "Minimizing occupational hazards in endoscopy: personal protective equipment, radiation safety, and ergonomics." Gastrointestinal endoscopy, vol. 72, no. 2, pp. 227-235, Aug. 2010. [Online]. Available: http://www.ncbi.nlm.nih.gov/pubmed/20537638

[24] N. Kuperij, R. Reilink, M. P. Schwartz, S. Stramigioli, S. Misra, and I. A. M. J. Broeders, “Design of a User Interface for Intuitive Colonoscope Control," in IEEE International Conference on Intelligent Robots and Systems 2011. San Francisco: IEEE, 2011, pp. 2076-2082. 
[25] B. P. Saunders, M. Fukumoto, S. Halligan, C. Jobling, M. E. Moussa, C. I. Bartram, and C. B. Williams, "Why is colonoscopy more difficult in women?" Gastrointestinal endoscopy, vol. 43, no. 2 Pt 1, pp. 124-6, Feb. 1996. [Online]. Available: http: //www.ncbi.nlm.nih.gov/pubmed/8635705

[26] D. Scaramuzza and F. Fraundorfer, "Visual Odometry: Part I - The First 30 Years and Fundamentals," IEEE Robotics and Automation Magazine, vol. 18, no. 4, pp. 80-92, 2011.

[27] F. Fraundorfer and D. Scaramuzza, "Visual Odometry: Part II - Matching, Robustness and Applications," IEEE Robotics and Automation Magazine, vol. 19, no. 2, pp. 78-90, 2012.

[28] D. Gillies and G. Khan, "Vision based navigation system for an endoscope," Image and Vision Computing, vol. 14, pp. 763-772, 1996.

[29] C. K. Kwoh, G. N. Khan, and D. F. Gillies, "Automated Endoscope Navigation and Advisory System from medical imaging," in SPIE's International Conference on Physiology and Function fro Multidimensional Images, vol. 3660. San Diego, CA: SPIE press, 1999, pp. 214-224.

[30] H. Chettaoui, G. Thomann, C. B. Amar, and T. Redarce, "Extracting and tracking Colon's "Pattern" from Colonoscopic Images," in IEEE Canadian Conference on Computer and Robot Vision. IEEE, 2006, pp. 65-71.

[31] I. Bricault, G. Ferretti, and P. Cinquin, "Registration of real and CT-derived virtual bronchoscopic images to assist transbronchial biopsy." IEEE transactions on medical imaging, vol. 17, no. 5, pp. 703-14, Oct. 1998. [Online]. Available: http://www.ncbi.nlm.nih. gov/pubmed/9874294

[32] R. Reilink, S. Stramigioli, and S. Misra, "Image-Based Flexible Endoscope Steering," in IEEE/RSJ International Conference on Intelligent Robots and Systems, no. i. Taipei, Taiwan: IEEE, 2010, p. 6.

[33] R. Reilink, S. Stramigioli, A. M. L. Kappers, and S. Misra, "Evaluation of flexible endoscope steering using haptic guidance." The international journal of medical robotics and computer assisted surgery, vol. 7, no. 2, pp. 178-86, Jun. 2011. [Online]. Available: http://www.ncbi.nlm.nih.gov/pubmed/21462290

[34] X. Zhiyun, "Computerized Detection of Abnormalities in Endoscopic Oesophageal Images," Master of Engineering, Nanyang Technological University, 2000.

[35] Z. Zhen, Q. Jinwu, Z. Yanan, and S. Linyong, "An Intelligent Endoscopic Navigation System," in IEEE International Conference on Mechatronics and Automation. Luoyang, China: leee, Jun. 2006, pp. 1653-1657. [Online]. Available: http://ieeexplore.ieee.org/ Ipdocs/epic03/wrapper.htm?arnumber $=4026339$

[36] S. Krishnan, C. Tan, and K. Chan, "Closed-boundary extraction of large intestinal lumen," Proceedings of 16th Annual International Conference of the IEEE Engineering in Medicine and Biology Society, pp. 610-611, 1994. [Online]. Available: http://ieeexplore.ieee.org/ Ipdocs/epic03/wrapper.htm?arnumber $=411878$

[37] M. P. Tjoa, S. M. Krishnan, and M. M. Zheng, "A Novel Endoscopic Image Analysis Approach using Deformable Region Model to Aid in Clinical Diagnosis," in Proc. 15th Ann. Int. Conf. of the IEEE EMBS, no. 2, Cancun, Mexico, 2003, pp. 710-713.

[38] S. Xia, S. M. Krishnan, M. P. Tjoa, and P. M. Y. Goh, "A Novel Methodology for Extracting Colon's Lumen from Colonoscopic Images," J Systemics, Cybernetics and Informatics, vol. 1, no. 2, pp. 7-12, 2003. [Online]. Available: http://www.iiisci.org/journal/CV\/sci/ pdfs/000380.pdf 
[39] J. Batlle, E. Mouaddib, and J. Salvi, "Recent progress in coded structured light as a technique to solve the correspondence problem: a survey," Pattern Recognition, vol. 31, no. 7, pp. 963-982, 1998.

[40] J. Salvi, J. Pagès, and J. Batlle, "Pattern codification strategies in structured light systems," Pattern Recognition, vol. 37, no. 4, pp. 827-849, Apr. 2004. [Online]. Available: http://linkinghub.elsevier.com/retrieve/pii/S0031320303003303

[41] G. Zhang, J. He, and X. Li, "3D vision inspection for internal surface based on circle structured light," Sensors and Actuators, vol. 122, no. 1, pp. 68-75, Jul. 2005. [Online]. Available: http://linkinghub.elsevier.com/retrieve/pii/S0924424705002347

[42] A. Mekaouar, C. Ben Amar, and T. Redarce, "New vision based navigation clue for a regular colonoscope's tip," Proceedings of SPIE, vol. 7261, pp. 72611B-72 611B-9, 2009. [Online]. Available: http://link.aip.org/link/PSISDG/v7261/i1/p72611B/s1\\&Agg=doi

[43] G. Ciuti, M. Visentini-scarzanella, A. Dore, A. Menciassi, P. Dario, and G.-Z. Yang, "Intraoperative Monocular 3D Reconstruction for Image-Guided Navigation in Active Locomotion Capsule Endoscopy," in IEEE RAS and EMBS International Conference on Biomedical Robotics and Biomechatronics (BioRob). Rome: IEEE, 2012, pp. 768-774.

[44] H. U. Rashid and P. Burger, "Differential algorithm for the determination of shape from shading using a point light source," Image and Vision Computing, vol. 10, no. 2, pp. 119127, 1992.

[45] N. Van der Stap, R. Reilink, S. Misra, I. A. M. J. Broeders, and F. van der Heijden, "The Use of the Focus of Expansion for Automated Steering of Flexible Endoscopes," in IEEE BioRob. Rome, Italy: IEEE, 2012, pp. 13-18. [Online]. Available: http://ieeexplore.ieee.org/lpdocs/epic03/wrapper.htm?arnumber=6290804

[46] N. Van der Stap, R. Reilink, S. Misra, I. Broeders, and F. Van der Heijden, "A feasibility study of optical flow-based navigation during colonoscopy," The International Journal of Computer Assisted Radiology and Surgery, vol. 7, no. S1, p. S235, 2012.

[47] N. Masson, F. Nageotte, P. Zanne, and M. D. Mathelin, "In vivo comparison of real-time tracking algorithms for interventional flexible endoscopy," in ISBI. Boston,MA,USA: IEEE, 2009, pp. 1350-1353.

[48] K. Deguchi, T. Sasano, H. Arai, and Y. Yoshikawa, "3-D shape reconstruction from endoscope image sequences by the factorization method," in IAPR Workshop on Machine Vision Applications (MVA'94), Kawasaki, 1994, pp. 455-459.

[49] C. Poelman and T. Kanade, "A paraperspective factorization method for shape and motion recovery," IEEE Transactions on Pattern Analysis and Machine Intelligence, vol. 19, no. 3, pp. 206-218, Mar. 1997. [Online]. Available: http://ieeexplore.ieee.org/lpdocs/epic03/ wrapper.htm?arnumber $=584098$

[50] D. Deguchi, K. Mori, Y. Suenaga, J.-I. Hasegawa, J.-I. Toriwaki, H. Natori, and H. Takabatake, "New calculation method of image similarity for endoscope tracking based on image registration in endoscope navigation," International Congress Series, vol. 1256, pp. 460-466, Jun. 2003. [Online]. Available: http://linkinghub.elsevier.com/retrieve/ pii/S0531513103003315

[51] D. Deguchi, K. Mori, M. Feuerstein, T. Kitasaka, C. R. Maurer, Y. Suenaga, H. Takabatake, M. Mori, and H. Natori, "Selective image similarity measure for bronchoscope tracking based on image registration." Medical image analysis, vol. 13, no. 4, pp. 621-33, Aug. 2009. [Online]. Available: http://www.ncbi.nlm.nih.gov/pubmed/19592291 
[52] S. Phee, W. Ng, I. Chen, F. Seow-Choen, and B. Davies, "Locomotion and steering aspects in automation of colonoscopy. Part one.A literature review." IEEE engineering in medicine and biology magazine: the quarterly magazine of the Engineering in Medicine \& Biology Society, vol. 16, no. 6, pp. 85-96, 1997. [Online]. Available: http://www.ncbi.nlm.nih.gov/pubmed/9399091

[53] J. Ruiter, E. Rozeboom, M. Van der Voort, M. Bonnema, and I. Broeders, "Design and Evaluation of Robotic Steering of a Flexible Endoscope," in IEEE BioRob. Roma, Italia: IEEE, 2012, pp. 761-767.

[54] K. Mori, D. Deguchi, J. Sugiyama, Y. Suenaga, J. Toriwaki, C. R. Maurer, H. Takabatake, and $\mathrm{H}$. Natori, "Tracking of a bronchoscope using epipolar geometry analysis and intensity-based image registration of real and virtual endoscopic images." Medical image analysis, vol. 6, no. 3, pp. 321-36, Sep. 2002. [Online]. Available: http: //www.ncbi.nlm.nih.gov/pubmed/12270236

[55] N. National Cancer Institute, "Colorectal cancer," p. 1, Nov. 2011. [Online]. Available: http://www.nlm.nih.gov/medlineplus/colorectalcancer.html

[56] B. Spier, M. Benson, P. Pfau, G. Nelligan, M. Lucey, and E. Gaumnitz, "Colonoscopy training in gastroenterology fellowships: determining competence," Gastrointestinal endoscopy, vol. 71, no. 2, pp. 319-324, Feb. 2010. [Online]. Available: http://linkinghub.elsevier.com/retrieve/pii/S0016510709019142?showall=truehttp: //www.ncbi.nlm.nih.gov/pubmed/19647242

[57] P. Tassios, S. Ladas, I. Grammenos, K. Demertzis, and S. Raptis, "Acquisition of competence in colonoscopy: the learning curve of trainees." Endoscopy, vol. 31, no. 9, pp. 702-6, Nov. 1999. [Online]. Available: http://www.ncbi.nlm.nih.gov/pubmed/10604610

[58] M. Liedlgruber and A. Uhl, "Endoscopic Image Processing - An Overview," in Proc. 6th symp. on Image and Signal Processing and Analysis. Salzburg: IEEE, 2009, pp. 707-712.

[59] K. Mori, D. Deguchi, J.-I. Hasegawa, and Y. Suenaga, "A Method for Tracking the Camera Motion of Real Endoscope by Epipolar Geometry Analysis and Virtual Endoscopy System," in MICCAI - Lecture Notes in Computer Science, vol. 2208. Springer-Verlag Berlin Heidelberg, 2001, pp. 1-8.

[60] W. Burger and B. Bhanu, "On Computing a 'Fuzzy' Focus of Expansion for Autonomous Navigation," in IEEE Conference on Computer Vision and Pattern Recognition, no. 3. Rosemont, IL, USA: IEEE, 1989, pp. 563-568.

[61] B. D. Lucas and T. Kanade, "An iterative image registration technique with an application to stereo vision," in International Joint Conference on Artificial Intelligence (IJCAI). San Francisco, CA, USA: Morgan Kaufmann Publishers Inc., 1981, pp. 674-679.

[62] B. K. Horn and B. G. Schunck, "Determining Optical Flow," in SPIE, vol. 0281. SPIE, 1981, pp. 185-203.

[63] D. G. D. Lowe, "Object Recognition from Local Scale-Invariant Features," in International Conference on Computer Vision, vol. 2. Corfu: IEEE, 1999, pp. 1150-1157. [Online]. Available: http://ieeexplore.ieee.org/lpdocs/epic03/wrapper.htm?arnumber=790410

[64] F. Van der Heijden, R. P. W. Duin, D. De Ridder, and D. M. J. Tax, Classification, Parameter Estimation and State Estimation. Chichester: John Wiley \& Sons Ltd, 2004.

[65] I. T. Jolliffe, Principal Component Analysis, Second Edition, 2nd ed., P. Bickel, P. Diggle, S. Fienberg, K. Krickeberg, I. Olkin, N. Wermuth, and S. Zeger, Eds. New York: SpringerVerlag New York, Inc., 2002. 
[66] M. A. Fischler and R. C. Bolles, "Random Sample Consensus: a paradigm for model fitting with applications to image analysis and automated cartography," Communications of the ACM, pp. 381-395, 1981.

[67] E. M. Jellinek, "On the use of the intra-class correlation coefficient in the testing of the difference of certain variance ratios," Journal of Educational Psychology, vol. 31, no. 1, pp. 60-63, 1940. [Online]. Available: http://dx.doi.org/10.1037/h0062703

[68] J. Shi and C. Tomasi, "Good features to track," in Proceedings CVPR '94. Seattle: IEEE, 1994, pp. 593-600.

[69] H. Bay, A. Ess, T. Tuytelaars, and L. Vangool, "Speeded-Up Robust Features (SURF)," Computer Vision and Image Understanding, vol. 110, no. 3, pp. 346-359, Jun. 2008. [Online]. Available: http://linkinghub.elsevier.com/retrieve/pii/S1077314207001555

[70] E. Rosten and T. Drummond, "Fusing points and lines for high performance tracking," ICCV '05, vol. 1, pp. 1508-1515, 2005. [Online]. Available: http://ieeexplore.ieee.org/lpdocs/ epic03/wrapper.htm?arnumber $=1544896$

[71] H. Longuet-Higgins and K. Prazdny, "The interpretation of a moving retinal image," in Proceedings of the Royal Society London, vol. 208. London: JSTOR, 1980, pp. 385-397. [Online]. Available: www.jstor.org

[72] H. Chao, Y. Gu, and M. Napolitano, "A survey of optical flow techniques for robotics navigation applications," Journal of Intelligent and Robotic Systems: Theory and Applications, vol. 73, no. 1-4, pp. 361-372, 2014.

[73] R. Hartley and A. Zisserman, Multiple View Geometry in Computer Vision, 2nd ed. New York, NY, USA: Cambridge University Press, 2003.

[74] J.-C. Zufferey and D. Floreano, "Toward 30-gram Autonomous Indoor Aircraft: Visionbased Obstacle Avoidance and Altitude Control," in Proceedings of the 2005 IEEE International Conference on Robotics and Automation. leee, 2005, pp. 2594-2599. [Online]. Available: http://ieeexplore.ieee.org/lpdocs/epic03/wrapper.htm?arnumber=1570504

[75] D. D. Doyle, A. L. Jennings, and J. T. Black, "Optical flow background estimation for real-time pan/tilt camera object tracking," Measurement, vol. 48, pp. 195-207, Feb. 2014. [Online]. Available: http://linkinghub.elsevier.com/retrieve/pii/S0263224113005241

[76] R. Carloni, V. Lippiello, M. D’Auria, M. Fumagalli, A. Y. Mersha, S. Stramigioli, and B. Siciliano, "Robot vision: Obstacle-avoidance techniques for unmanned aerial vehicles," IEEE Robotics and Automation Magazine, vol. 20, no. 4, pp. 22-31, 2013.

[77] N. van der Stap, C. Slump, I. Broeders, and F. van der Heijden, "Image-based Navigation for a Robotized Flexible Endoscope," Lecture Notes in Computer Science, vol. 8899, pp. 77-87, 2014.

[78] A. Bruhn and J. Weickert, "Towards ultimate motion estimation: Combining highest accuracy with real-time performance," Proceedings of the IEEE International Conference on Computer Vision, vol. I, pp. 749-755, 2005.

[79] J. L. Barron, D. J. Fleet, and S. S. Beauchemin, "Performance of Optical Flow Techniques," International Journal of Computer Vision, vol. 12, no. 1, pp. 43-77, 1994.

[80] C. McCarthy and N. Barnes, "Performance of optical flow techniques for indoor navigation with a mobile robot," IEEE International Conference on Robotics and Automation, 2004. Proceedings. ICRA '04. 2004, vol. 5, no. April, pp. 5093-5098, 2004. 
[81] S. Baker, D. Scharstein, J. P. Lewis, S. Roth, M. J. Black, and R. Szeliski, "A database and evaluation methodology for optical flow," International Journal of Computer Vision, vol. 92, no. 1, pp. 1-31, 2011.

[82] B. McCane, K. Novins, D. Crannitch, and B. Galvin, "On Benchmarking Optical Flow," Computer Vision and Image Understanding, vol. 84, no. 1, pp. 126-143, Oct. 2001. [Online]. Available: http://linkinghub.elsevier.com/retrieve/pii/S1077314201909300

[83] N. van der Stap, F. van der Heijden, and I. A. M. J. Broeders, "Towards automated visual flexible endoscope navigation," Surgical Endoscopy, vol. 27, no. 10, pp. 3539-47, 2013.

[84] C. S. Bell, K. L. Obstein, and P. Valdastri, "Image partitioning and illumination in image-based pose detection for teleoperated flexible endoscopes," Artificial Intelligence In Medicine, vol. 59, no. 3, pp. 185-196, 2013. [Online]. Available: http://dx.doi.org/10.1016/ j.artmed.2013.09.002

[85] C. S. Bell, G. A. Puerto, G.-L. Mariottini, and P. Valdastri, "Six DOF Motion Estimation for Teleoperated Flexible Endoscopes Using Optical Flow: A Comparative Study," in IEEE International Conference on Robotics \& Automation. Hong Kong: IEEE, 2014, pp. 5386-5392. [Online]. Available: https://my.vanderbilt.edu/stormlab/files/2014/06/ ICRA2014ᄂBell.pdf

[86] L. Liu, C. Hu, W. Cai, and M. Q. H. Meng, "Capsule endoscope localization based on computer vision technique." in Annual International Conference of the IEEE Engineering in Medicine and Biology Society., vol. 2009. IEEE, Jan. 2009, pp. 3711-4. [Online]. Available: http://www.ncbi.nlm.nih.gov/pubmed/19965009

[87] M. Lerotic, A. J. Chung, J. Clark, S. Valibeik, and G.-Z. Yang, "Dynamic view expansion for enhanced navigation in Natural Orifice Transluminal Endoscopic Surgery." in Int. Conf. on medical image computing and computer-assisted interventions: MICCAI, vol. 11, no. Pt 2, Jan. 2008, pp. 467-75. [Online]. Available: http://www.ncbi.nlm.nih.gov/pubmed/ 18982638

[88] F. Selka, S. A. Nicolau, V. Agnus, A. Bessaid, J. Marescaux, and L. Soler, "Evaluation of Endoscopic Image Enhancement for Feature Tracking: A New Validation Framework," in MIAR/AE-CAI LNCS, C. Linte, Ed., no. 8090. Springer-Verlag Berlin Heidelberg, 2013, pp. 77-85.

[89] R. Jimbo, J. Fernandez-Rodriguez, Y.-T. Sul, and C. B. Johansson, "Principal Component Analysis: A Novel Analysis to Evaluate the Characteristics of Osseointegration of Different Implant Surfaces." Implant dentistry, pp. 1-6, Jul. 2011. [Online]. Available: http://www.ncbi.nlm.nih.gov/pubmed/21893521

[90] J.-Y. Bouguet, "Pyramidal Implementation of the Lucas Kanade Feature Tracker - Description of the algorithm," Intel Corporation, Tech. Rep. 2, 2003.

[91] H.-H. Nagel, "On the estimation of optical flow: Relations between different approaches and some new results," Artificial Intelligence, vol. 33, no. 3, pp. 299-324, 1987.

[92] D. G. Lowe, "Distinctive Image Features from Scale-Invariant Keypoints," International Journal of Computer Vision, vol. 60, no. 2, pp. 1-28, 2004.

[93] M. Grabner, H. Grabner, and H. Bischof, "Fast approximated SIFT," in Computer Vision ACCV 2006, 2006, ch. Fast Appro, pp. 918-927.

[94] A. Alahi, R. Ortiz, and P. Vandergheynst, "FREAK: Fast Retina Keypoint," in 2012 IEEE Conference on Computer Vision and Pattern Recognition. IEEE, 2012, pp. 510-517. [Online]. Available: http://ieeexplore.ieee.org/lpdocs/epic03/wrapper.htm?arnumber=6247715 
[95] E. Rublee, V. Rabaud, K. Konolige, and G. Bradski, "ORB: An efficient alternative to SIFT or SURF," 2011 International Conference on Computer Vision, pp. 2564-2571, Nov. 2011. [Online]. Available: http://ieeexplore.ieee.org/lpdocs/epic03/wrapper.htm? arnumber $=6126544$

[96] Y. Ma, S. Soatto, S. Sastry, and J. Kosecka, An Invitation to 3-D Vision: From Images to Models, 26th ed., S. Antman, J. Marsden, L. Sirovich, and S. Wiggins, Eds. New York, NY, USA: Springer Science + Business Media LCC, 2006.

[97] Z. Zhang, "Determining the Epipolar Geometry and its Uncertainty: A Review," International Journal of Computer Vision, vol. 27, no. 2, pp. 161-195, 1998. [Online]. Available: http://dx.doi.org/10.1023/a:1007941100561

[98] S. Metkar and S. Talbar, "Performance Evaluation of Block Matching Algorithms for Video Coding," in Motion Estimation Techniques for Digital Video Coding, 2013, ch. 2, pp. 13-31. [Online]. Available: http://link.springer.com/10.1007/978-81-322-1097-9

[99] P. Allemann, L. Ott, M. Asakuma, N. Masson, S. Perretta, B. Dallemagne, D. Coumaros, M. De Mathelin, L. Soler, and J. Marescaux, "Joystick interfaces are not suitable for robotized endoscope applied to NOTES." Surgical innovation, vol. 16, no. 2, pp. 111-6, Jun. 2009. [Online]. Available: http://www.ncbi.nlm.nih.gov/pubmed/19546121

[100] B. Bardou, F. Nageotte, P. Zanne, and M. de Mathelin, "Improvements in the control of a flexible endoscopic system," 2012 IEEE International Conference on Robotics and Automation, pp. 3725-3732, May 2012. [Online]. Available: http://ieeexplore.ieee.org/ Ipdocs/epic03/wrapper.htm?arnumber $=6225050$

[101] N. van der Stap, L. Voskuilen, G. De Jong, H. Pullens, I. Schwartz, M.P. Broeders, and F. van der Heijden, "A Real-Time Target Tracking Algorithm for a Robotic Flexible Endoscopy Platform," in 2nd workshop on Computer-Assisted and Robotized Endoscopy, T. Reichl, G.-L. Mariottini, and X. Luo, Eds. Munich, Germany: Springer, 2015.

[102] H. J. M. Pullens, N. van der Stap, E. D. Rozeboom, M. P. Schwartz, F. van der Heijden, M. G. H. V. Oijen, P. D. Siersema, and I. A. M. J. Broeders, "Colonoscopy with robotic steering and automated lumen centralization: a feasibility study in a colon model," Endoscopy, vol. eFirst ahe, 2015. [Online]. Available: https://www.thieme-connect.com/DOI/DOI?10.1055/ s-0034-1392550

[103] G. Chen, G. Thomann, M. T. Pham, M. Bbtemps, T. Redarce, and I. D. Lyon, "Modeling and Control of a Colonoscopic Tip under the Disturbance of the Insertion of Colonoscope," in Intelligent Robots and Systems. IEEE, 2004, pp. 3315-3320.

[104] A. J. Davison, I. D. Reid, N. D. Molton, and O. Stasse, "MonoSLAM: real-time single camera SLAM." IEEE transactions on pattern analysis and machine intelligence, vol. 29, no. 6, pp. 1052-67, Jun. 2007. [Online]. Available: http://www.ncbi.nlm.nih.gov/pubmed/17431302

[105] J.-Y. Bouguet, "Camera Calibration Toolbox for Matlab," 2013. [Online]. Available: http://www.vision.caltech.edu/bouguetj/calib\_doc/index.html\\#ref

[106] S. Stramigioli, Modeling and IPC control of interactive mechanical systems - A coordinatefree approach, ser. Lecture Notes in Control and Information Sciences. London: Springer London, 2001, vol. 266. [Online]. Available: http://link.springer.com/10.1007/BFb0110400

[107] R. Taylor, "A Perspective on Medical Robotics," Proceedings of the IEEE, vol. 94, no. 9, pp. 1652-1664, Sep. 2006. [Online]. Available: http://ieeexplore.ieee.org/lpdocs/epic03/ wrapper.htm?arnumber $=1717783$ 
[108] R. P. W. Duin and D. M. J. Tax, "PRTools - A Matlab toolbox for pattern recognition," 2013. [Online]. Available: http://prtools.org

[109] J. S. Terhaar sive Droste, M. E. Craanen, J. J. Kolkman, and C. J. J. Mulder, "Dutch endoscopic capacity in the era of colorectal cancer screening," Netherlands Journal of Medicine, vol. 64, no. 10, pp. 371-373, 2006.

[110] J. B. Marshall, "Technical proficiency of trainees performing colonoscopy: A learning curve," Gastrointestinal Endoscopy, vol. 42, no. 4, pp. 287-291, 1995

[111] S. T. Ward, M. A. Mohammed, R. Walt, R. Valori, T. Ismail, and P. Dunckley, "An analysis of the learning curve to achieve competency at colonoscopy using the JETS database." Gut, pp. 1-9, 2014. [Online]. Available: http://www.ncbi.nlm.nih.gov/pubmed/24470280

[112] W. Ridtitid, G. A. Coté, W. Leung, R. Buschbacher, S. Lynch, E. L. Fogel, J. L. Watkins, G. A. Lehman, S. Sherman, and L. McHenry, "Prevalence and risk factors for musculoskeletal injuries related to endoscopy," Gastrointestinal Endoscopy, vol. 81, no. 2, pp. 294-302.e4, 2014. [Online]. Available: http://dx.doi.org/10.1016/j.gie.2014.06.036

[113] S. H. Zhang, D. X. Wang, Y. R. Zhangl, Y. H. Wang, Y. G. Wang, and X. P. Maz, "The Human machine Interface Implementation for the Robot assisted Endoscopic Surgery System," in Int. Workshop on Robot and Human Interactive Communication. Berlin, Germany: IEEE, 2002, pp. 442-447.

[114] A. M. Plooy, A. Hill, M. S. Horswill, A. S. G. Cresp, M. O. Watson, S.-Y. Ooi, S. Riek, G. M. Wallis, R. Burgess-Limerick, and D. G. Hewett, "Construct validation of a physical model colonoscopy simulator." Gastrointestinal endoscopy, vol. 76, no. 1, pp. 144-50, Jul. 2012. [Online]. Available: http://www.ncbi.nlm.nih.gov/pubmed/22726473

[115] I. M. Gralnek, D. L. Carr-Locke, O. Segol, Z. Halpern, P. D. Siersema, A. Sloyer, J. Fenster, B. S. Lewis, E. Santo, A. Suissa, and M. Segev, "Comparison of standard forward-viewing mode versus ultrawide-viewing mode of a novel colonoscopy platform: a prospective, multicenter study in the detection of simulated polyps in an in vitro colon model (with video)." Gastrointestinal endoscopy, vol. 77, no. 3, pp. 472-9, Mar. 2013. [Online]. Available: http://www.ncbi.nlm.nih.gov/pubmed/23410700

[116] E. D. Rozeboom, J. G. Ruiter, M. Franken, and I. A. Broeders, "Intuitive user interfaces increase efficiency in endoscope tip control." Surgical endoscopy, vol. 28, no. 9, pp. 2600-5, Mar. 2014. [Online]. Available: http://www.ncbi.nlm.nih.gov/pubmed/24671354

[117] R. Siegel, C. Desantis, and A. Jemal, "Colorectal cancer statistics, 2014." CA: a cancer journal for clinicians, vol. 64, no. 2, pp. 104-17, 2014. [Online]. Available: http://www.ncbi.nlm.nih.gov/pubmed/24639052

[118] P. Autier, "Colorectal Cancer Screening Works; 'Irrefutable' Evidence that Fall in Death Rates is Attributable to Screening Programmes," 2013. [Online]. Available: http://www. esmo.org/Conferences/Past-Conferences/European-Cancer-Congress-2013/News/

[119] G. Geers and E. van der Vlis - Vester, "Screen - special darmkanker," Regionale voorlichtingsbijeenkomst darmkanker bevolkingsonderzoek, Amsterdam, The Netherlands, Tech. Rep., 2012

[120] A. K. Shergill, K. R. McQuaid, and D. Rempel, "Ergonomics and Gl endoscopy," Gastrointestinal endoscopy, vol. 70, no. 1, pp. 145-53, Jul. 2009. [Online]. Available: http://www.ncbi.nlm.nih.gov/pubmed/19559836

[121] R. L. Barclay, J. J. Vicari, A. S. Doughty, J. F. Johanson, and R. L. Greenlaw, "Colonoscopic withdrawal times and adenoma detection during screening colonoscopy." The New 
England journal of medicine, vol. 355, no. 24, pp. 2533-41, Dec. 2006. [Online]. Available: http://www.ncbi.nlm.nih.gov/pubmed/17167136

[122] K. L. Obstein and P. Valdastri, "Advanced endoscopic technologies for colorectal cancer screening." World journal of gastroenterology: WJG, vol. 19, no. 4, pp. 431-9, Jan. 2013. [Online]. Available: http://www.ncbi.nlm.nih.gov/pubmed/23382621

[123] K. V. Asari, "A fast and accurate segmentation technique for the extraction of gastrointestinal lumen from endoscopic images." Medical engineering \& physics, vol. 22, no. 2, pp. 89-96, Mar. 2000. [Online]. Available: http://www.ncbi.nlm.nih.gov/pubmed/ 10854962

[124] S. Hansel, J. Prechel, B. Horn, M. Crowell, and J. DiBaise, "Observational study of the frequency of use and perceived usefulness of ancillary manoeuvres to facilitate colonoscopy completion." Digestive and liver disease, vol. 41, no. 11, pp. 812-6, Nov. 2009. [Online]. Available: http://www.ncbi.nlm.nih.gov/pubmed/19467939

[125] S. Shah, B. Saunders, J. Brooker, and C. Williams, "Magnetic imaging of colonoscopy: an audit of looping, accuracy and ancillary maneuvers." Gastrointestinal endoscopy, vol. 52, no. 1, pp. 1-8, Jul. 2000. [Online]. Available: http://www.ncbi.nlm.nih.gov/pubmed/ 10882954

[126] J. G. Ruiter, M. C. van der Voort, and M. G. Bonnema, "User-centred system design approach applied on a robotic flexible endoscope," in Procedia Computer Science - 2013 Conference on Systems Engineering Research, C. Paredis, C. Bishop, and D. Bodner, Eds., vol. 16. Atlanta: Elsevier Inc., 2013, pp. 581-590.

[127] E. Rozeboom, I. Broeders, and P. Fockens, "Feasibility of joystick guided colonoscopy; assessing the learning curves of experts and novices," Journal of Robotic Surgery, 2015. [Online]. Available: "http://dx.doi.org/10.1007/s11701-015-0511-6

[128] E. Rozeboom, J. Ruiter, M. Franken, M. Schwartz, S. Stramigioli, and I. Broeders, "Single-handed controller reduces the workload of flexible endoscopy," Journal of Robotic Surgery, vol. 8, no. 4, pp. 319-324, 2014. [Online]. Available: http://link.springer.com/10. 1007/s11701-014-0473-0

[129] Python Software Foundation, "Python v2.7 documentation," 2014. [Online]. Available: https://docs.python.org/2.7/

[130] OpenCV Development Team, "OpenCV documentation," 2014. [Online]. Available: http: //docs.opencv.org/

[131] L. Ott, F. Nageotte, P. Zanne, M. D. Mathelin, and S. Member, "Robotic Assistance to Flexible Endoscopy by Physiological-Motion Tracking," IEEE Transactions on Robotics, vol. 27, no. 2, pp. 346-359, 2011.

[132] S. Atasoy, D. Mateus, A. Meining, G.-Z. Yang, and N. Navab, "Endoscopic video manifolds for targeted optical biopsy." IEEE transactions on medical imaging, vol. 31, no. 3, pp. 637-53, Mar. 2012. [Online]. Available: http://www.ncbi.nlm.nih.gov/pubmed/22057050

[133] B. Allain, M. Hu, L. Lovat, R. Cook, T. Vercauteren, S. Ourselin, and D. Hawkes, "Re-localisation of a biopsy site in endoscopic images and characterisation of its uncertainty." Medical image analysis, vol. 16, no. 2, pp. 482-96, Feb. 2012. [Online]. Available: http://www.ncbi.nlm.nih.gov/pubmed/22197442

[134] M. Ye, S. Giannarou, N. Patel, and J. Teare, "Pathological Site Retargeting under Tissue Deformation Using Geometrical Association," in MICCAI 2013, Part II, LNCS, K. Mori, Ed., vol. 8150. Heidelberg, Germany: Springer-Verlag Berlin, 2013, pp. 67-74. 
[135] J. Liu, B. Wang, W. Hu, Y. Zong, J. Si, and H. Duan, "A non-invasive navigation system for retargeting gastroscopic lesions." Bio-medical materials and engineering, vol. 24, no. 6, pp. 2673-9, Jan. 2014. [Online]. Available: http://www.ncbi.nlm.nih.gov/pubmed/25226971

[136] Y.-J. Chu, S.-P. Liu, R. C. Luo, R.-H. Hu, C.-C. Yeh, Y. W. Peng, and P.-L. Yen, “Dynamic Tracking of Anatomical Object for a Steerable Endoscope," in International Conference on Advanced Intelligent Mechatronics, 2012.

[137] S. Speidel and S. Krappe, "Robust feature tracking for endoscopic pose estimation and structure recovery," in Proc. SPIE, vol. 8671. SPIE digital library, 2013, pp. 1-7. [Online]. Available: http://proceedings.spiedigitallibrary.org/proceeding.aspx?articleid=1663315

[138] Z. Yaniv and K. Cleary, "Image-guided procedures: A review," Georgetown University CAIMR, Washington, DC, Tech. Rep. April, 2006. [Online]. Available: http://www.caimr. georgetown.edu/

[139] T. Rösch, A. Adler, H. Pohl, E. Wettschureck, M. Koch, B. Wiedenmann, and N. Hoepffner, "A motor-driven single-use colonoscope controlled with a hand-held device: a feasibility study in volunteers." Gastrointestinal endoscopy, vol. 67, no. 7, pp. 1139-46, Jun. 2008. [Online]. Available: http://www.ncbi.nlm.nih.gov/pubmed/18355823

[140] D. J. Mirota, H. Wang, R. H. Taylor, M. Ishii, and G. D. Hager, "Toward video-based navigation for endoscopic endonasal skull base surgery," in MICCAI 2009, 2009.

[141] E. Spyrou and D. K. lakovidis, "Homography-based orientation estimation for capsule endoscope tracking," in IST 2012 - 2012 IEEE International Conference on Imaging Systems and Techniques, Proceedings, no. September 2015, 2012, pp. 101-105.

[142] E. Spyrou and D. Iakovidis, "Video-based Measurements for Wireless Capsule Endoscope Tracking," Measurement Science and Technology, vol. 25, no. 1, p. 14, 2014.

[143] R. El-Hawary and A. Popovic, "Robust feature tracking on the beating heart for a roboticguided endoscope," The international journal of medical robotics + computer assisted surgery, vol. 7, pp. 459-468, 2011.

[144] B. Zheng, Z. Janmohamed, and C. L. MacKenzie, "Reaction times and the decision-making process in endoscopic surgery: An experimental study," Surgical Endoscopy and Other Interventional Techniques, vol. 17, no. 9, pp. 1475-1480, 2003.

[145] J. Tian, H. S. Ying, and D. S. Zee, "Revisiting corrective saccades: Role of visual feedback," Vision Research, vol. 89, pp. 54-64, 2013. [Online]. Available: http: //dx.doi.org/10.1016/j.visres.2013.07.012

[146] E. D. Rozeboom, R. Reilink, M. P. Schwartz, P. Fockens, and I. A. Broeders, "Evaluation of tip bending response in clinically used endoscopes," 2015.

[147] L. Ott, P. Zanne, F. Nageotte, M. de Mathelin, and J. Gangloff, "Physiological motion rejection in flexible endoscopy using visual servoing," 2008 IEEE International Conference on Robotics and Automation, pp. 2928-2933, May 2008. [Online]. Available: http://ieeexplore.ieee.org/lpdocs/epic03/wrapper.htm?arnumber=4543654

[148] N. Masson, F. Nageotte, P. Zanne, M. De Mathelin, and J. Marescaux, "Comparison of visual tracking algorithms on in vivo sequences for robot-assisted flexible endoscopic surgery," in Engineering in Medicine and Biology Society, 2009. Minneapolis, MN: IEEE, 2009, pp. 5571-5576.

[149] B. Bardou, F. Nageotte, P. Zanne, and M. de Mathelin, "Design of a telemanipulated system for transluminal surgery." in IEEE Engineering in Medicine and Biology Society, vol. 2009, Jan. 2009, pp. 5577-82. [Online]. Available: http://www.ncbi.nlm.nih.gov/pubmed/ 19964131 
[150] V. Agrawal, W. J. Peine, and B. Yao, "Modeling of a closed loop cable-conduit transmission system," in IEEE International Conference on Robotics and Automation, ICRA. IEEE, 2008, pp. 3407-3412.

[151] --, "Modeling of transmission characteristics across a cable-conduit system," IEEE Transactions on Robotics, vol. 26, no. 5, pp. 914-924, 2010.

[152] D. B. Camarillo, C. F. Milne, C. R. Carlson, M. R. Zinn, and J. K. Salisbury, "Mechanics modeling of tendon-driven continuum manipulators," IEEE Transactions on Robotics, vol. 24, no. 6, pp. 1262-1273, 2008. 


\section{About the author}

Nanda van der Stap was born in the Western part of the Netherlands (Heemstede, Noord-Holland) in 1985. She went to high school at College Hageveld, which was a monumental building surrounded by woods. She was most interested in the subjects of biology and physics. Technical Medicine at the University of Twente, a campus which is also surrounded by woods, therefore was the right choice from the beginning. Of course, this choice meant moving across the country.

In addition to being active for all kinds of activities around Technical Medicine (TA at several courses, member of the Student Evaluation Committee, Student employee at the Experimental Centre for Technical Medicine), she managed to finish the study as well. She obtained her Bachelor's degree in 2008 and her Master's degree in 2011.

She had already started to work on her PhD subject by then. The research was carried out at the same University of Twente, but also at the Meander Medical Centre in Amersfoort. This led to another move, this time to the center of the country.

During her PhD she published several papers, most of which can be found in this dissertation, and contributed to a number of conferences. Most notable among these are the MICCAI, BioRob and SRS international conferences. She was an invited speaker at a few Dutch symposia as well as an invited student at summer- and winter schools at Imperial College in London and at the IRCAD in Strassbourg, among others.

As an honorable appreciation of her work, she was awarded the Poster Prize at the annual Meander Scientific Symposium in 2015 as well as the Best Paper Award at the 2014 MICCAI workshop on Computer-Assisted and Robotized Endoscopy. Last but not least, her name was on the 2015 'Nerd 101' list of most notable technologists in the Netherlands. This list was assembled by 'Vrij Nederland', a Dutch opinion magazine.

Currently, Nanda holds a position in the Netherlands as research scientist at the technological innovation company TNO. 


\section{Dankwoord}

Bij een proefschrift hoort een dankwoord als een promotor bij een promotie. Voor mij was die promotor Ivo (dat mag ik zeggen inmiddels), of Professor Broeders, zoals de meeste Technische Geneeskunde (TG) studenten hem kennen. Echter, jou slechts mijn 'promotor' noemen zou je tekort doen. Je was en bent in meerdere opzichten een mentor voor mij. In het ziekenhuis leerde je mij enorm veel over de chirurgie, maar ook de medische wereld in het algemeen. Op de UT leerde je mij wat het inhoudt om een promotietraject te doorlopen, zowel de zware aspecten (accepteren dat je resultaten niet bruikbaar zijn of je artikel herschreven moet worden) als de leuke (met name de congresbezoeken staan in mijn geheugen gegrift). Ook had je vaak wat algemene levenswijsheden, zoals dat een goed onderhandelaar het emotionele en het zakelijke aspect altijd loskoppelt. lets dat mij slechts sporadisch lukt (maar jou ook, vertelde je). Over de wijze lessen van het laparoscopisch hechten zal ik maar niet in dit proefschrift reppen... Nog als de dag van gisteren herinner ik me mijn eerste afspraak bij jou. Ik was vergeten het één en ander uit te printen, maar wilde ook niet te laat komen. De oplossing? Snel op tijd je kantoor binnenstormen, nog net zeggen: 'Ik moet nog even iets printen, ik kom zo terug!', om je vervolgens ongetwijfeld licht verontwaardigd achter te laten. Mocht ik het nog niet hebben gezegd toen: mijn excuses voor deze stormachtige en éénzijdige kennismaking. Gelukkig is het vrij snel daarna helemaal goed gekomen, tenminste, dat leidde ik af uit het feit dat je mij een promotieplek aanbood... 1,5 jaar vóórdat ik zou afstuderen. Na een bedenktijd van ongeveer een half jaar besloot ik in te gaan op je aanbod, en ik heb er (echt!) nooit spijt van gehad. Nadat ik mijn draai had gevonden lukte het me zelfs om vaker dan eens per twee maanden met je af te spreken, en hebben we volgens mij een mooie periode gehad. Niet alleen hebben we samen geschitterd in een aflevering voor het TV-programma 'Chirurgenwerk', ook hebben we vaker succesvolle gezamenlijke besprekingen gehouden, samen wintersportvakanties met de chirurgie overleefd (met name de après-ski was zwaar) en samen natuurlijk meermaals ons werk gepubliceerd en gepresenteerd. Kortom, door al deze aspecten heb je mijn promotie een rijke belevenis gemaakt en daarvoor ben en blijf ik je dankbaar.

Wat niet bij elke promotie hoort, maar gelukkig wel bij deze, is een copromotor. Een chirurg als promotor, hoe technisch geïnteresseerd ook, zorgde ervoor dat ik nog wat extra technische begeleiding nodig had, en die vond ik bij Ferdi. Ik kende je vanuit de opleiding TG, waar ik jouw colleges altijd op de voet volgde (echt!) en ik houd je dan ook verantwoordelijk voor mijn fascinatie voor het gebruiken van wiskunde voor de analyse van afbeeldingen. Gelukkig stond je open voor het vervullen van de rol van copromotor, maar ook jij bent meer dan dat. Er zijn hele perioden geweest dat je iets was dat nog het meest in de buurt kwam van een 'dagelijks begeleider', met name voor de deadlines van conferenties. Ik kon je altijd bereiken, je stond altijd klaar. Je hebt me heel veel geleerd op het gebied van computer vision en mijn fascinatie hiervoor tijdens mijn promotie alleen maar aangewakkerd. Je kon ook altijd honderduit praten over jouw fascinatie: het zeilen. Naast dat je dit veelvuldig als voorbeeld in je onderwijs gebruikt, vergeet ik ook nooit dat je heel enthousiast in je vakantie liet weten dat je momenteel langs het Noordwijkse strand zeilde, op weg naar Londen (ik zat toen op het strand voor de Noordwijkse Reddingsbrigade). Niet minder enthousiast heb ik gezwaaid, al betwijfel ik of je dat kon zien. Ik geef toe, ik 
moest wel even wennen aan onze samenwerking. In het begin kon ik niet goed inschatten of een stilte van jouw kant betekende dat ik iets heel doms of juist iets zinnigs had gezegd. Later begreep ik dat het meestal betekende dat je even nadacht over het zojuist gezegde. Volgens mij zijn onze afspraken over de jaren heen inhoudelijk steeds dieper gegaan en daarmee dus ook zinniger geworden. Het begeleiden van studenten vond ik altijd een feest, en hoewel we samen wat speciale gevallen hebben begeleid vond ik het altijd leuk om te merken dat we elkaar daar goed in aanvulden en vaak op één lijn zaten. Je blijft altijd rustig, maar kan ook ongezouten je mening geven, en juist die combinatie vind ik zo fascinerend. Natuurlijk kan ik ook ons gezamenlijk musiceren in de enige echte RaMband niet vergeten, en onze twee optredens waren een belevenis die niet iedereen met zijn of haar copromotor zal delen. Dankjewel dus!

Mijn lieve paranimfen Esther (bijgestaan door Eddy) en Wendie (bijgestaan door Arne), zonder jullie was deze promotie er nooit gekomen. En nee, dan bedoel ik niet alleen de dag zelf, al waardeer ik alle moeite die jullie gedaan hebben voor bijvoorbeeld de uitnodigingen ook héél erg, maar ik bedoel de promotie als geheel. Wendie, al sinds ik in 2004 bij jou in huis kwam wonen kan ik me niet meer voorstellen dat ik dingen níet met je deel. Gelukkig wonen we ook in Utrecht niet meer dan 10 minuten bij elkaar vandaan, anders had ik vast ontwenningsverschijnselen gekregen. Ik ben zó blij dat ik je kan opbellen als er iets is, maar ook als er niets is, met je kan afspreken om hard te lopen, maar ook om heel veel lekkere dingen te eten, maar vooral dat je altijd klaarstaat als er iets is (een ad-hoc IKEA bezoek of een tijdelijke her-inwoning bijvoorbeeld. Arne, met name dit laatste geldt ook voor jou!). Zonder deze momenten had ik het ongetwijfeld niet gered de afgelopen vier jaar, en ik hoop dat we nog héél lang op deze manier met elkaar om blijven gaan. Niet vergeten: you're my person!

Esther, jou ken ik iets minder lang, maar we hebben zó intensief samengewerkt de afgelopen drie jaar dat ook jij een onmisbaar element van deze promotie bent. De gezellige momenten waarop we even samen 'lekkere' koffie gingen halen, wat eigenlijk een goed excuus was om lekker bij te kletsen over persoonlijke zaken én om over al onze collega's te kunnen roddelen (sorry jongens...), hebben me menige ochtend doorgesleept. En natuurlijk: al onze reizen samen voor congressen! Gek op de foto in de dierentuin of een bierfestival crashen in Frankfurt, overleven in een zeer krakkemikkige hotelkamer in Praag, elkaar wakker houden tot het avondprogramma in Straatsburg, Sachertorte (de echte!) eten in Wenen, we deden het allemaal en ik denk er met plezier aan terug. Op een iets serieuzer vlak ben ik ook heel blij dat we elkaar professioneel gezien zo goed aanvullen, en ik denk dat allebei onze promotietrajecten hier beter van zijn geworden. Met name daarom waardeer ik het ook extra dat er nu minstens twee papers bestaan waarboven onze beide namen prijken. Ik ben blij dat ik jou en Eddy ook een klein beetje heb kunnen helpen met het opbouwen van jullie prachtige huisje, en ik wens jullie daar ontzettend veel plezier en geluk toe. Die dates in Den Haag, Utrecht en Enschede komen vast ook helemaal goed!

Stefano, dit houd ik kort, want ik weet dat je weinig tijd hebt, haha. We kennen elkaar van de studiereis in Brazilië in 2008. Ik organiseerde hem, jij ging mee als begeleiding, en volgens mij hebben we een hele mooie tijd gehad! Daarna raakte ik geïnteresseerd in robotica, en doordat ik niet alle vakken chronologisch kon volgen vanwege diezelfde reis vroeg ik of je misschien een leuke opdracht voor me had. Als gewoonlijk reageerde je enthousiast, je bracht me in contact met Ivo, en de rest is geschiedenis. Toen onze vakgroepen fuseerden in 2014 zijn we weer intensiever gaan samenwerken, met als mooie hoogtepunten natuurlijk het oprichten van de RaMband en het binnenhalen van de H2020 grant voor MURAB. Dankjewel!

Professor Slump, Kees, naast de dank voor het plaatsnemen in de commissie wilde ik je graag bedanken voor het invallen toen Ferdi op zomervakantie was. We besloten vrij acuut dat we tóch een bijdrage wilden leveren aan het MICCAI congres, en dat betekende een week lang bikkelen om het paper op tijd af te krijgen. Niet voor niets gelukkig, hij werd geaccepteerd en 
won later zelfs een best paper award! Zonder jouw inspanningen en verbeteringen was dit niet gelukt, dankjewel.

Graag wil ik de andere collega's bij RAM (en daarvoor ook SAS) bedanken voor de prettige samenwerking, de mooie tripjes (brainstorms, EuRoC) en de gezellige tijd in het algemeen. Jolanda en Sandra, bedankt voor alle dingen die jullie altijd wisten te regelen en de gezellige theemomenten tussendoor. Roomies, thanks voor het lachen, de lunches en de slingers!

Dear Silvana, thank you for taking part in the committee today, and for responding so enthusiastically when we asked you. Through some coincidence we met in Strassbourg a few years ago, and together with Vivian we had a great time. Since then we meet regularly at conferences, company visits and of course your great B.E.S.T. initiative. You are a great inspiration to me, for multiple reasons, and I wish you all the best!

Pieter Dik, ik mocht het er niet meer over hebben, maar toch bedankt voor je-weet-wel-wat. Daarnaast vond ik het ontzettend leuk en onverwacht dat je wilde komen opponeren vandaag, en ik hoop dat we elkaars enthousiasme voor slimme oplossingen in de toekomst nog vaak mogen aanwakkeren!

De andere twee leden van de commissie, prof. Niessen en prof. Ruers, ken ik meer zijdelings via TG en een congres in Boston, maar natuurlijk ben ik ook erg dankbaar dat u hier wilde plaatsnemen en alle moeite wilde doen voor dit proefschrift.

Een groot deel van mijn promotie bracht ik ook door in het ziekenhuis van Amersfoort, het Meander Medisch Centrum. Met een aantal mensen heb ik heel intensief samengewerkt. Een aantal waren net als ik bezig met een promotietraject, wat in de praktijk betekent dat je met zijn allen 'vast' zit achter je computer in de overdrachtsruimte. Bryan en Henk, dank voor de mooie tijden! Marguerite, met jou heb ik intensiever samengewerkt, ben zelfs op je bruiloft geweest. Heerlijk konden we kletsen over van alles, soms over teveel, want er moest ook nog wel het één en ander gebeuren aan werk. Hoewel we over ons werk ook genoeg discussieerden, van paperopbouw tot statistische details. Ik hoop dat jij ook binnenkort je promotie kunt afronden, hoewel dit door je mooie plek bij de VU natuurlijk wat extra moeite kost. Extra veel geluk daarom met alles, en dankjewel dat je de integrerende factor wilde zijn tussen TGers en assistenten chirurgie.

Jacqueline, als OK manager en trekker van het robotproject kwam ik jou vaak tegen, en eigenlijk klikte het vanaf het begin goed. Nicole heeft ons in contact gebracht toen zij en ik aan het afstuderen waren, en hoewel Nicole besloot een andere weg in te slaan zijn wij blijven samenwerken. Met veel plezier! Eén van de hoogtepunten was toch wel Athene voor mij, maar ik waardeerde de koffiemomenten tussendoor als we incognito in het restaurant konden zitten ook heel erg. Toen je tijdens één van die sessies de foto's van de bruiloft van je dochter liet zien viel me gelijk op dat dat helemaal mijn smaak foto's was. Gekscherend zei je: 'Ik doe nooit meer bruidsreportages, maar voor jouw bruiloft maak ik een uitzondering!'. In het kort kan ik nu zeggen: heel erg bedankt voor de leuke tijd, maar speciaal voor de foto's van vandaag (bruiloft, promotie, bijna hetzelfde, toch?)!

Wie Jacqueline zegt, zegt Peter, in ieder geval in 'mijn tijd' in het Meander. Samen zaten we in de commissie nieuwbouw, maar je was ook mijn steun en toeverlaat voor het organiseren van de Robotdagen voor TG studenten. Ik heb altijd met veel plezier met je samengewerkt, dus dankjewel! En ook voor de bloemen ja!

Tijdens mijn hele periode in het Meander heb ik de mogelijkheid gehad om in de operatiekamer hele indrukwekkende nieuwe technieken te mogen zien, testen, ermee te mogen spelen en werken, en daarnaast om altijd overal mee te kijken. Zonder het OK team (in het bijzonder Perla en Rianne) en de planners had dit alles niet gekund, dus jullie ook bedankt! Het bijbehorende onderwijs kreeg ik veelal van Ivo zelf, maar ook vaak genoeg van één van zijn collega's. Van de 'robotchirurgen' heb ik het vaakst kennis mogen aftappen, en daarvoor wil ik Esther, Werner en Paul erg danken. Ook voor alle gezelligheid, niet alleen rond de OK maar ook op de legendarische 
wintersporten natuurlijk. Dokters Van Overbeeke, Van Ooijen, Dijkema, Verberne, De Vries, Van Olden, Verheijen, Verweij, Voorhoeve, ik wil jullie bedanken voor het opnemen van zo'n 'rare' TGer in jullie team!

Niet alleen op de OK heb ik mogen meekijken en leren, ook op de endoscopie/MDL afdeling werd ik altijd met open armen ontvangen. In het bijzonder is met Thijs en Paul een zeer vruchtbare samenwerking ontstaan. 'Vruchtbaar', omdat het geresulteerd heeft in meerdere papers samen, maar bijvoorbeeld ook in een plekje voor Thijs tussen de opponenten, wat ik heel bijzonder en leuk vind. Jullie moeten wel veel passie voor je vak hebben, dat je naast de drukke patiëntenzorg ook nog dingen als een promotietraject of een Stichts Genootschap organiseert, en ik bewonder dat enorm. Heel erg bedankt voor de prettige samenwerking, hopelijk kunnen we dat voortzetten! Daarnaast wil ik ook de andere MDL-artsen graag bedanken, omdat zij allemaal mee hebben gedaan aan experimenten, maar ook allemaal TG studenten hebben ontvangen, en ook allemaal ideeën opperen voor het verbeteren van ons systeem. Jullie zijn top, bedankt!

De derde partij die onmisbaar was voor deze promotie (naast universiteit en ziekenhuis), was het bedrijfsleven, en dan specifiek de bedrijven Demcon en Olympus. Met Demcon is het originele voorstel voor het Teleflex project, waaruit de robot gebouwd is, ingediend. Rob, Michel en Benno, jullie kende ik allemaal op een andere manier en al sinds mijn studietijd, maar jullie zijn allemaal bij Demcon gaan werken in de loop van mijn promotie en zo bleven we met elkaar te maken hebben. Wat ik alleen maar leuk vond! Jeroen is zelf gepromoveerd op het Teleflex project en we hebben een prachtig congres in Rome samen meegemaakt, dank voor de prettige samenwerking. Dennis ken ik natuurlijk als de grote baas, al was Michiel meer de 'dagelijkse baas' voor mij. Heren, met jullie allemaal heb ik zeer prettig samengewerkt in de afgelopen jaren. Volgens mij hebben we elkaar goed kunnen helpen en ik hoop dat we dit in de toekomst kunnen blijven doen. Wie Olympus zegt binnen dit project zegt John van Wezel, die vele deuren voor ons heeft geopend. Bedankt voor al je snelle reacties en je enthousiasme over dit project, en laten we de samenwerking vooral in stand houden!

Natuurlijk was dit alles ook niet gelukt zonder familie en vrienden. Pap en mam, ik ben jullie dankbaar voor teveel om op te noemen, jullie staan altijd voor me klaar. Ook al zeggen jullie altijd dat jullie toch niet snappen waar ik me nu mee bezighoud, elke dag heb ik profijt van de dingen die ik van jullie leerde, en die jullie dus duidelijk wel goed hebben begrepen. Voorbeelden? Wees altijd beleefd, maar een af en toe gezond gebrek aan respect voor autoriteit maakt je niet te bang om je mond open te trekken. Zorg dat je er altijd netjes uitziet, maar wees wel praktisch in wat je aantrekt (naaldhakken zijn heel mooi, maar kun je beter thuis laten als je de duinen in gaat. Of de hele dag moet staan op een congres). Misschien nog wel de belangrijkste van deze voorbeelden: doe vooral wat je leuk vindt, maar het zou fijn zijn als je er ook van kunt leven. Het werk dat ik nu doe vind ik fantastisch, dus dank voor alle zetjes in de goede richting!

Allerliefste zus, we hebben vaak en veel contact, en jouw onverminderde positiviteit en steun (uit het niets over de app: 'Je kunt het zus! Laatste loodjes!') hebben me echt geholpen. Als we afspreken is dat meestal spontaan en gaan we eten, high-teaen of wandelen. Als we het pad kunnen vinden tenminste... Zelfs sleepovers zitten gelukkig nog in ons repertoire, net als 'vroeger'. $\mathrm{Nu}$ is dat echter wel iets minder praktisch doordeweeks, aangezien we de dag erna toch echt gewoon moeten werken... Samen kunnen we heerlijk lachen (meestal om jouw imitaties), ziekenhuisperikelen doornemen en elkaar daarbij helpen of praten over belangrijke levenskeuzes (of gewoon: 'wat zal ik vanavond aan doen naar dat feestje?'). We delen lief en leed en je staat altijd voor me klaar. Ik hoop dat je dit andersom ook zo ervaart, en dat we dat nog lang mogen doen!

Allerliefste broer, wij hebben wat minder contact, deels door je spannende buitenland-avonturen, maar desalniettemin kunnen we volgens mij altijd bij elkaar terecht als dat nodig is. Hoewel je ook wel eens je vraagtekens zet bij wat ik nu precies doe ('Ik heb maar gezegd dat je iets met operatierobots doet, is dat goed?'), vind ik het heerlijk om lekker met jou te brainstormen over 
technische problemen. Jouw kennis zit meer in de praktijk, mijne meer in de theorie, maar zolang we elkaar net als op de middelbare school met mijn 5-VWO wiskunde (jij was toen... 12?) ergens in het midden vinden, zie ik ons voorlopig nog niet uitgepraat zijn. Enne... zolang jij over de hele wereld woont, heb ik genoeg vakantiebestemmingen!

Opa, en in gedachten natuurlijk ook oma, een betere opa kan ik me niet wensen. Vroeger heb ik veel tijd bij jullie doorgebracht, en ik heb er prachtige herinneringen aan over gehouden. U heeft me veel wijze lessen geleerd, soms met een knipoog, maar altijd nuttig. Vooral tips over hoe met geld om te gaan, 'wie je kent is belangrijker dan wie je bent' en goed ondernemerschap hebben me geen windeieren gelegd. In de toekomst hoop ik nog een eigen bedrijf op te mogen zetten, en de trots waarmee u praat over 'de zaak' overtuigt me ervan dat dat een goed idee is. Maar niet alleen met uw wijsheid en uw tijd bent $u$ gul, $u$ heeft er ook deels voor gezorgd dat ik mijn studie kon betalen op de momenten dat ik er niet naast kon werken (tijdens ziekenhuisstages). Nooit zal ik ook lekkerdere appeltaart proeven dan degene die u bakt! En dat schijnt u dan weer van mij geleerd te hebben... Kortom: ik hoop dat we elkaar nog lang dingen mogen leren, en ben dankbaar voor onze tijden samen.

Toen ik begon met studeren in Enschede waren er nog niet zoveel vrouwen als nu. En wat doe je dan? Je gaat bij een 'vrouwenclubje', want je wilt gewoon één keer in de week wél kunnen praten over 'vrouwendingen'. In mijn geval was dat Nefertiti (toen maar liefst 9 leden ongeveer?), een onafhankelijk damesdispuut uit Enschede (sinds 2004). Wat ik zo bijzonder vind aan deze club is dat we allemaal heel anders zijn, maar toch hetzelfde. Allemaal lief en ambitieus, misschien hier en daar een beetje gek, inmiddels verspreid door het hele land maar nog steeds doen we veel samen. Afstuderen, verjaardagen, festivals, borrelen, lunchen, eten, sporten: door jullie is zowel mijn tijd in Enschede (later ook met de Doabo) als in Utrecht (Vondelutregrotjeleiduh) een stuk leuker geworden! Dankjulliewel meiden, ook voor alle steun!

In Utrecht zijn er meer mensen die het leven leuker maken, en mijn promotie daardoor hebben gesteund. Vooral Ivo en Géraldine wil ik even bedanken; voor de gezellige feestjes, het autoadvies en de geslaagde verhuizing. De woonkamer zal nooit meer hetzelfde zijn! Ook in Enschede is er een vriendengroep die vooral het laatste deel van mijn promotie voor support en gezelligheid zorgde, al heb ik die een beetje 'geleend' van Stefan. Jullie ook allemaal bedankt!

Zeer recent ben ik begonnen te werken in Den Haag, en hoewel we elkaar nog niet zolang kennen, wil ik toch even zeggen dat ik enorm waardeer hoe ik ben opgevangen door alle collega's bij II. Toch een nieuw pad, een grote stap, een nieuwe stad, etc., maar jullie maken het wel heel makkelijk om eraan te wennen!

Veel steun, verspreid door het land dus, maar zelfs over de hele wereld. Esther, Jantine, Patricia en Tessa: al op Hageveld waren we een hecht clubje, en ik kan soms niet geloven dat we nog zó close zijn na al die jaren. Afgelopen juni zelfs een prachtig hoogtepunt met Tessa\&Calins bruiloft, en dat terwijl we echt een briljant vermogen hebben om zo vér mogelijk bij elkaar vandaan te gaan wonen. Was het eerst Leiden, Utrecht, Haarlem, Groningen en Enschede, nu is het zelfs Haarlem, Heemstede, Utrecht, Brisbane en L.A.. En nog steeds appen we elke week. Zoals Jantine ooit zei op haar afstuderen: 'Tja, dit is gewoon voor altijd'. Ik hoop dat echt, dankjulliewel!

En ik zei het al: Stefan. Natuurlijk zou ik jou niet vergeten. Je was er mijn hele promotie bij, maar in het begin wat meer op afstand dan aan het eind... Natuurlijk ben je een enorm deel van mijn leven, dus ook van mijn promotie, en daarvoor ben ik immens dankbaar. We kunnen door onze gezamenlijke interesses en 'nerdheid' heel wat leuke dingen samen doen, en ik hoop dat dat nog héél lang zo blijft! Toch heb je ook een hele specifieke bijdrage geleverd aan deze promotie, want ik weet zeker dat ik zonder jouw nuchtere opmerkingen, je humor, het feit dat je in dezelfde situatie zit als ik, je liefde, je eeuwige bereidheid om te luisteren (ook als ik aan het piepen ben) en je gezonde gekkigheid, mijn hoofd niet koel had kunnen houden tijdens de afronding van dit proefschrift. In één woord: dankjewel! 

